\title{
A CITY IS NOT A TREE
}


You are no doubt wondering by now what a city looks like which is a semilattice, but not a tree. I must confess that I cannot yet show you plans or sketches. It is not enough merely to make a demonstration of overlap - the overlap must be the right overlap. This is doubly important because it is so tempting to make plans in which overlap occurs for its own sake. This is essentially what the high- density 'life-filled' city plans of recent years do. But overlap alone does not give structure. It can also give chaos. A garbage can is full of overlap. To have structure, you must have the right overlap, and this is for us almost certainly different from the old overlap which we observe in historic cities. As the relationships between functions change, so the systems which need to overlap in order to receive these relationships must also change. The recreation of old kinds of overlap will be inappropriate, and chaotic instead of structured.

- Christopher Alexander, A city is not a tree (1965) 


\section{A CITY IS NOT A TREE MICHAEL BRADLEY RICHARDS}

A 120 - point thesis

submitted to the Victoria University of Wellington in partial fulfilment of the requirements for the

degree of Master of Architecture (Professional)

\section{Victoria University of Wellington}

School of Architecture 


\begin{abstract}
This research investigates the generation of urban environments through the use of visual programming tools. These tools enable the procedural creation of architectural geometries for installation in design environments, with a focus on producing outputs for virtual implementation, including small scale built environments, individual buildings, city blocks, and neighbourhoods

The creation of large-scale urban environments is a complex and time-consuming task. Subsequently, this field of research has a high level of relevance in the areas of architecture, design, urban planning, film, entertainment, and so forth. In most situations, the groups responsible for the creation of these environments do not contain members with architectural backgrounds. Instead, they consist of designers, computer scientists, technicians, and other specialists. These groups are reliant on their collective experience, skill, and reference materials to create their works.
\end{abstract}

This thesis proposes that architects possess knowledge, skills, and training suitable for utilisation by this industry. As such, this research explores applying an architectural education with a greater multidisciplinary focus.

This investigation concludes that while visual programming tools are incredibly powerful, they have their limitations. This research further concludes that because there are so many facets to the creation of these environments this area of investigation is best suited to a team of researchers. While individuals can achieve a significant amount, the contribution of outside parties would have had benefits at every stage of the work. The sharing of knowledge, skills, and understandings would allow for the creation of systems that function to generate the best outcomes possible. 


\section{ACKNOWLEDGMENTS}

Mum, Dad, and Natalie; I would not have finished this without your love, constant support, and housing.

Eliot, Jeff, and James; Our weekly meetings were hugely appreciated, even if I was consistently late. Along with everyone else in the studio, your presence and chats have made this experience worthwhile.

Emma, you have been a voice of reason for me this past year, and your advice has always pointed me in the right direction.

Tane Moleta, thank you for your time, criticism, ideas, off-topic conversations, and encouragement. You have been invaluable in completing this body of work. 
PREFACE

A CITY IS NOT A TREE 


\section{CONTENTS}

CHAPTER ONE

001

INTRODUCTION

CHAPTER TWO

013

PRELIMINARY DESIGN

CHAPTER THREE

EARLY CITY DEFINITIONS

039

CHAPTER FOUR

SINGLE CITY BLOCK ARTICULATION

047

CHAPTER FIVE

CITY BLOCK EXTRUSION

080

CHAPTER SIX

CITY SCALE DEFINITIONS

CONCLUSION

119

BIBLIOGRAPHY

103

121 


\section{CHAPTER ONE




\section{INTRODUCTION}

This research focuses on the practical application of visual-programming software as a means of generating authentic architectural geometries.

This research has been undertaken because the relevance and range of practical applications for computational design and programming to the architecture profession are rapidly increasing. These tools have applications ranging from workflow optimisation, to form generation, fabrication, and beyond. There is also an increasing necessity within the industry to understand these algorithmic design tools, to remain both competitive and relevant (Liao, 2015). On the matter, Nathan Miller (2016) wrote:

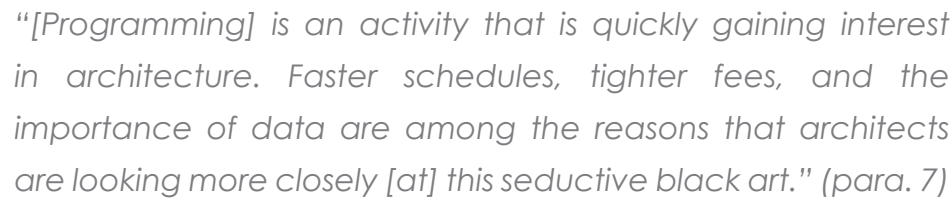

"[Programming] is an activity that is quickly gaining interest in architecture. Faster schedules, tighter fees, and the importance of data are among the reasons that architects are looking more closely [at] this seductive black art." (para. 7)

Compared to text-based programming visual-programming has a low barrier to entry, aiding its popularity and growing prevalence within the industry. Furthermore, these tools provide the promise of unique and exciting architectural outcomes that will make any firm stand out from the competition.
GRASSHOPPER

Grasshopper is reportedly the most popular visual-programming interface (Kilkelly, 2016). A 2014 analysis of 928 architectural jobs listings detailed that $3 \%$ percent of these listings required experience in the visual programming interface called Grasshopper. The next closest visual programming interface software was Digital Project at 1\% (Quirk, 2013). Other tools, such as Dynamo and Flux, are growing in popularity but do not share the same name recognition, or the number of users. Addressing the application of Grasshopper in this research, the following criticisms received consideration:

1) There is the possibility that a different software package may be more appropriate for generating specific geometries. While this may be true in some situations, Grasshopper is more than capable of generating these geometries and performing many other functions at the same time.

2) As a tool, Grasshopper is often incorrectly perceived as a fast and easy means of producing visually exciting geometries. Regarding the application of computational design in architecture, Michael Kilkelly (2016) wrote:

\footnotetext{
"Each step in the design becomes a series of instructions that can be evaluated, revised, and improved. Likewise, each step requires specific parameters. By thinking through all the steps of the design problem and considering all the inputs and outputs, you effectively create a process that can be understood and repeated." (para. 5)
}

3) Contrasting the perception that Grasshopper is simple, visualisations of the canvas can add to the complexity of any given project. These images are detrimental and do not provide useful information to the reader, regardless of their familiarity with Grasshopper.

4) For certain tasks software or tools could be constructed to perform roles exactly as required. Creation of these tools would take place in a way similar to how larger groups approach shading tools, rendering engines, and so on. However, it falls outside the scope and capabilities of this research. To create such a specific system would require a large team to be viable. 
There are many different methods for communicating information in Grasshopper, as in any other software. This research argues that three primary methods could be utilised to document the work:

Method 01: The actions can be written and explained in layman's terms. For example:

A rectangle is formed, with equal width and depth. The rectangle is then extruded five units in the Z-Direction. The resultant rectangular volume is moved in series nine further times in the Z-Direction, to create a series of stacked rectangular volumes,

Method 02: Raw Grasshopper canvas screenshot(s) (Fig. 1.01)

Method 03: Commutative diagram(s) (Fig. 1.02)

\begin{tabular}{|c|c|c|}
\hline Method & Positive & Negative \\
\hline 01 & $\begin{array}{c}\text { Communicates the actions, } \\
\text { exactly as they take place }\end{array}$ & $\begin{array}{c}\text { Significant number of words. Difficult } \\
\text { to communicate more complex } \\
\text { ideas and actions }\end{array}$ \\
\hline 02 & $\begin{array}{c}\text { Fast to create. Can be } \\
\text { interesting for those familiar } \\
\text { with Grasshopper }\end{array}$ & $\begin{array}{c}\text { Hard for anyone who is unfamiliar } \\
\text { with Grasshopper to understand. } \\
\text { Generally hard to read because of } \\
\text { size }\end{array}$ \\
\hline 03 & $\begin{array}{c}\text { No bias. Communicates } \\
\text { the actions very clearly. No } \\
\text { language or visual barriers }\end{array}$ & $\begin{array}{c}\text { Takes longer than the other } \\
\text { methods to create. Runs the } \\
\text { risk of oversimplifying complex } \\
\text { developments }\end{array}$ \\
\hline
\end{tabular}

Method 01 is suitable for this scale of work, and Method 02 (Fig. 1.02) can also be easily understood. However, this is a result of the canvas organisation and only 12 components being present. In his research, Daniel Davis established that the average Grasshopper canvas contains 23 components, with the complexity of any Grasshopper system growing in a positive linear manner with the number of nodes placed (2013). Regardless of Method 01 or 02's simplicity, Method 03 presents the outcome of this process in a barrierless manner. This selection ensures that the outputs display in a way that does not reveal any of the designer's programming or workflow preferences, biases, workarounds, and so forth. The process is made understandable to entire professions of individuals who are capable of comprehending standard architectural modes of communication.

Of note, the Grasshopper work presented within this research contains seemingly outlandish quantities of components, the number of which increases exponentially towards the conclusion. These systems involve anywhere from hundreds to hundreds of thousands of individual nodes. These figures are the result of the lack of any native looping functionality within Grasshopper and the avoidance of Grasshopper plugins such as Anemone or Hoopsnake.

In several instances throughout this work, clusters have been constructed to facilitate a single looping action. These groups of components lay in sequence with multiple duplicates of themselves, become linked and then controlled through logic gates. This process establishes a clunky but functional looping system, which allows for procedural modelling to take place. Although each cluster may be relatively simple in its actions and processes, it would be unreasonable to communicate this information through the employment of Method 01 or 02. On the following pages, Figures 1.03 (Page 007) and 1.04 (Page 008) make it very clear why these alternative methods of communicated would not be appropriate. 


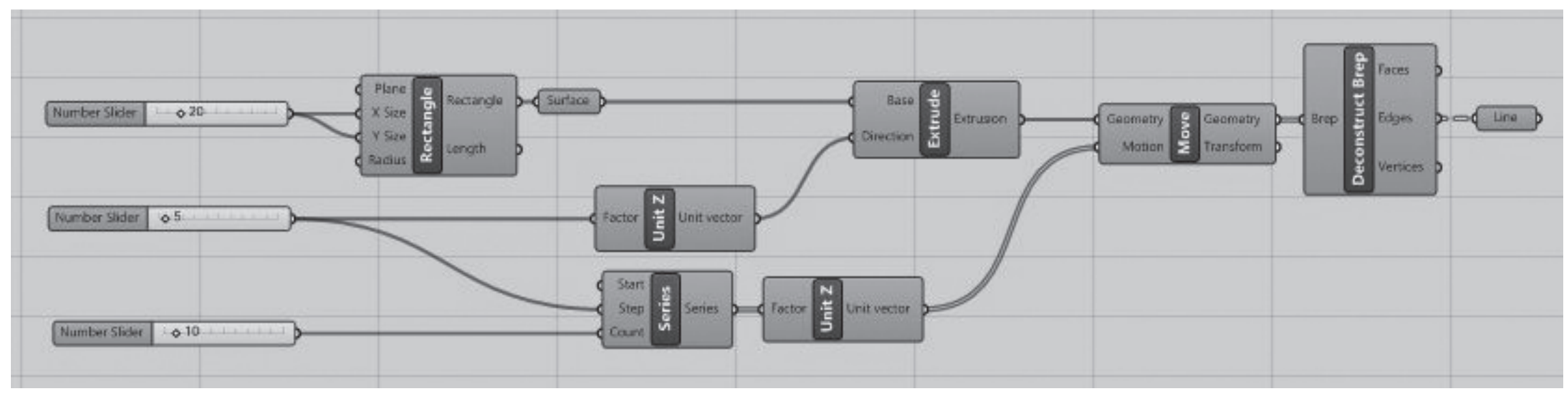

Figure 1.01: Image from Grasshopper canvas

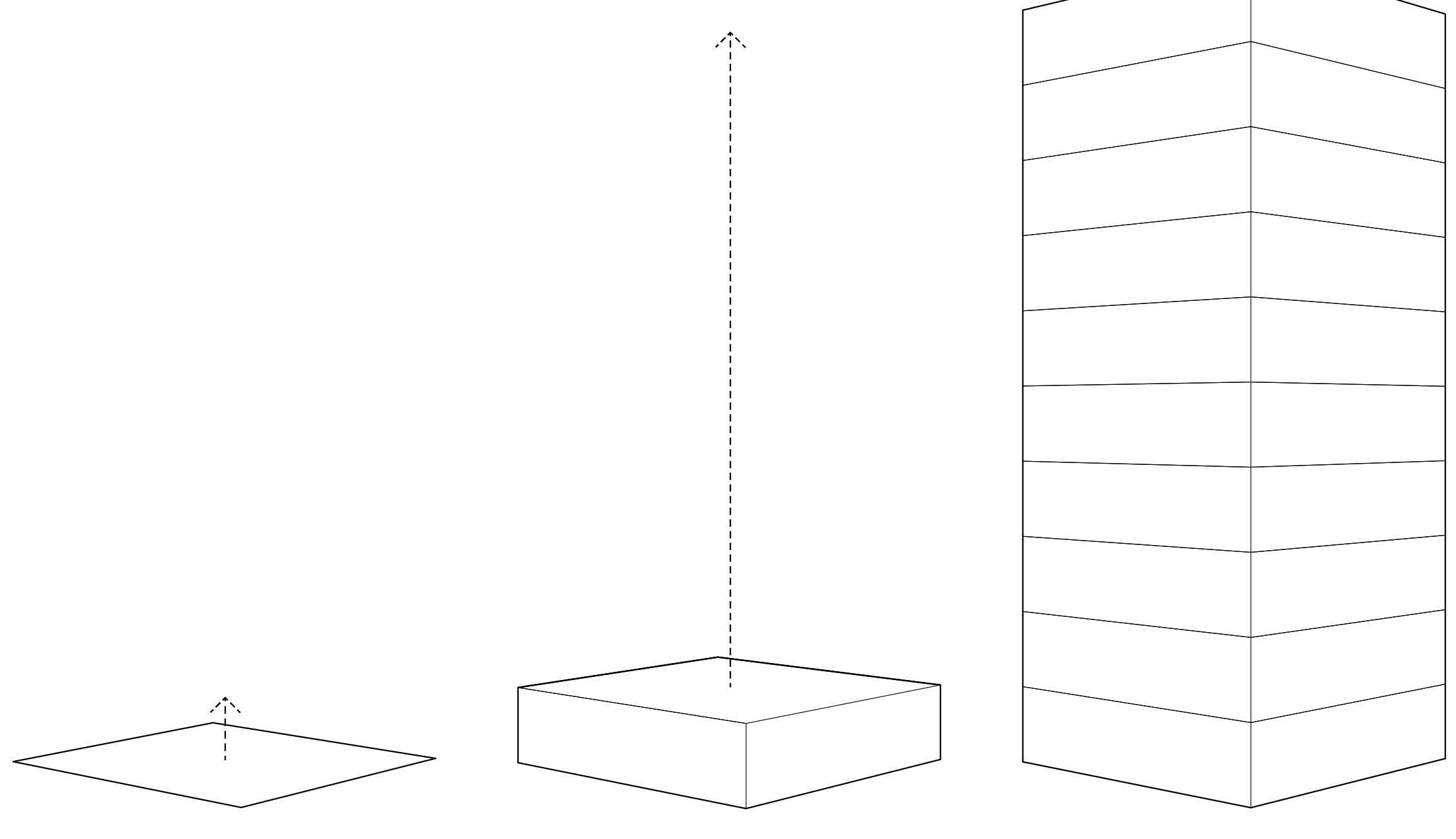

Figure 1.02: Grasshopper canvas visualised as communicative diagram 


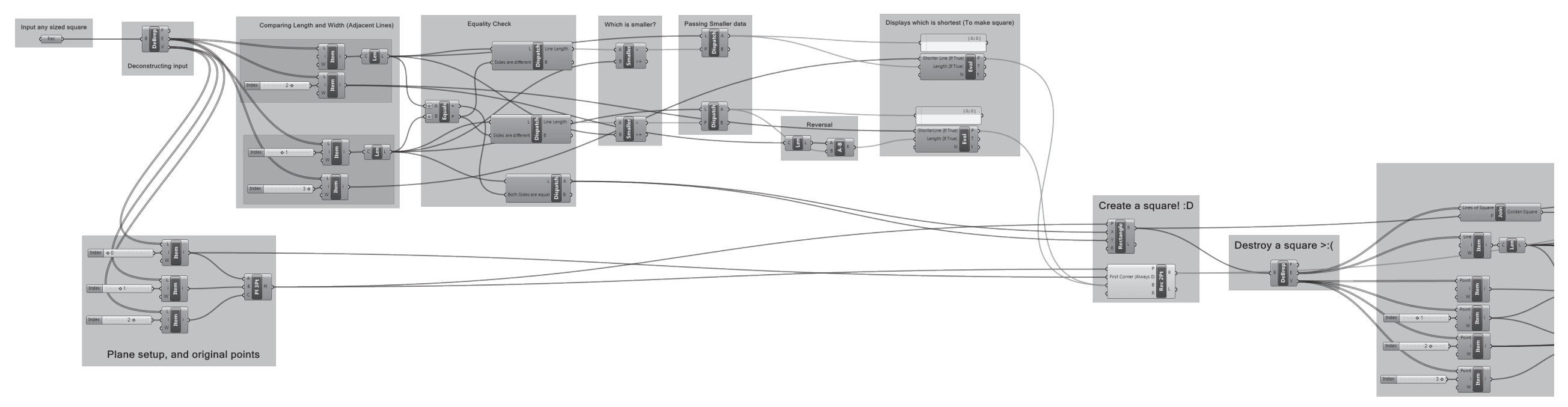

Figure 1.03: Analysis of First Grasshopper canvas

CHAPTER ONE

A CITY IS NOT A TREE 

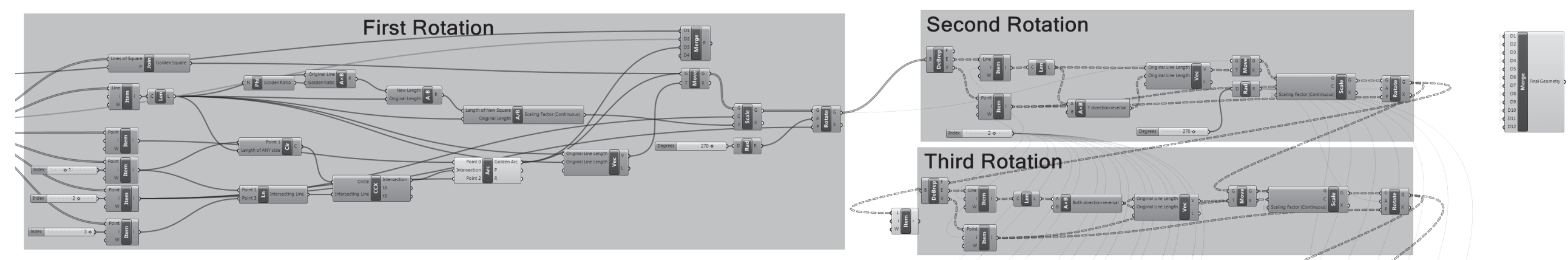

Fourth Rotation

需

Fifth Rotation

4.

Iixth

Sixth Rotation

II:

Seventh Rotation

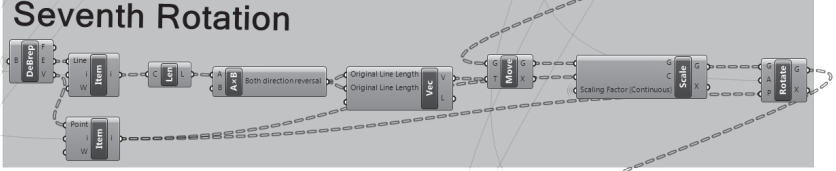

Eighth Rotation

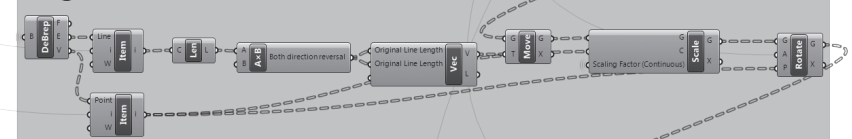

Ninth Rotation

Ninth Rotation

Tenth Rotation

II

Overall canvas contains 192 components

Figure 1.03: Analysis of First Grasshopper canvas (cont.) 


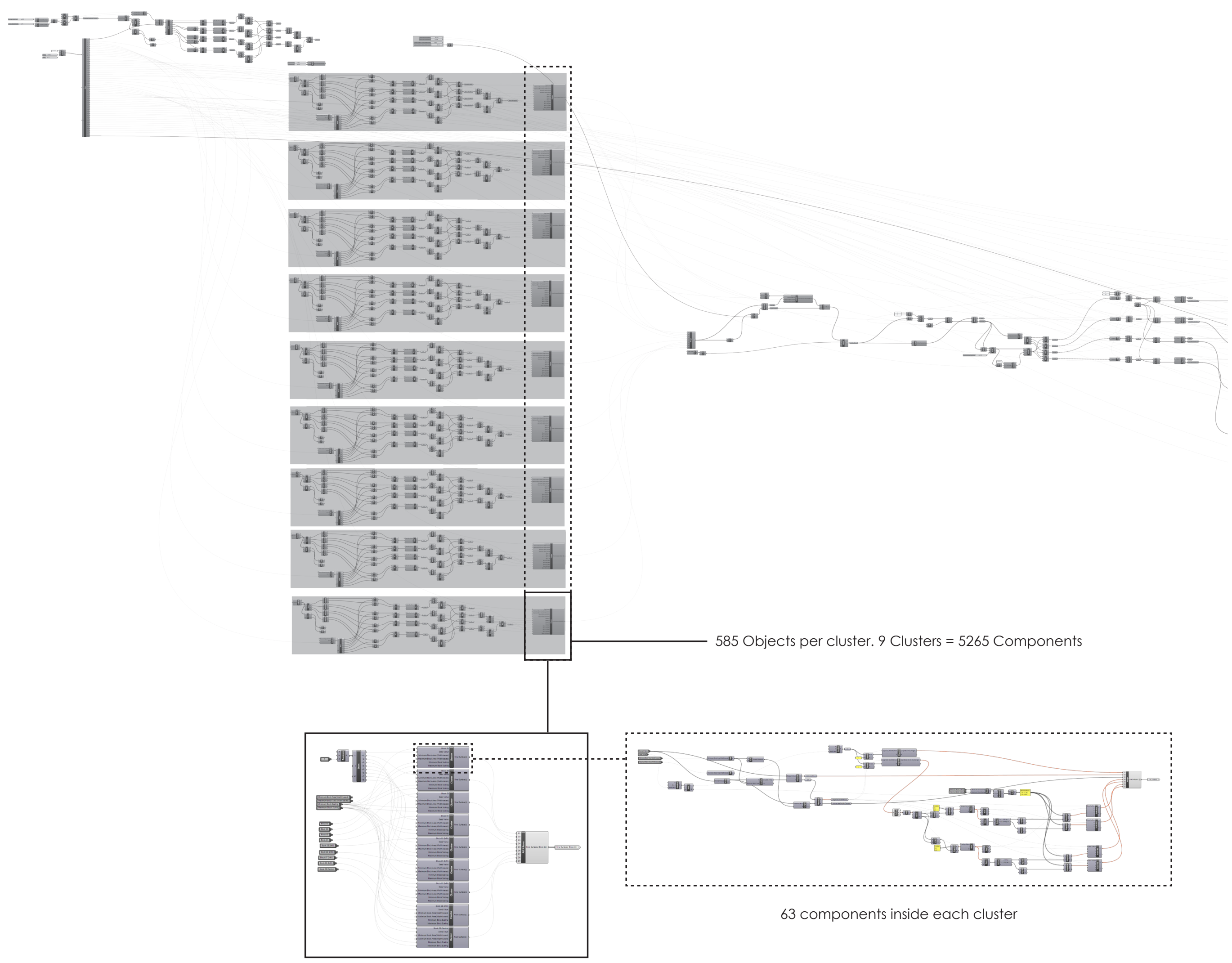

27 Objects, (9 Clusters + 18 Components)

Figure 1.04: Analysis of final Grasshopper canvas

CHAPTER ONE

A CITY IS NOT A TREE 


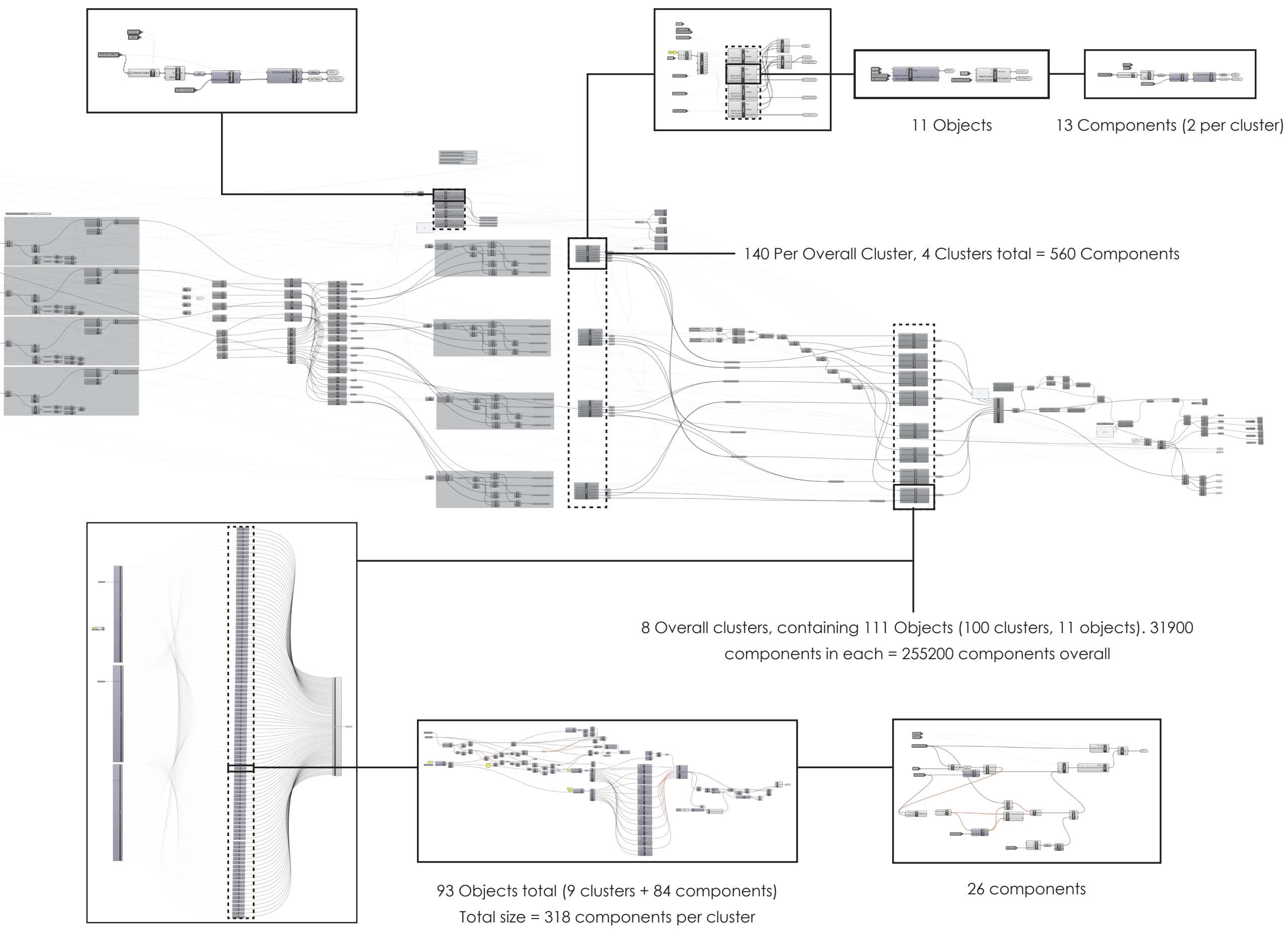

Overall canvas contains 948 objects ( 25 clusters +923 components) $=261948$ Components (Estimated)

Figure 1.04: Analysis of final Grasshopper canvas (cont.) 


\section{RESEARCH SCOPE}

This research aims to the investigate possible applications of parametric and procedural design techniques, within an architectural context. As such, the scope of this work encompasses the design of elements at a range of scales, extending from the micro through to macro to generate outputs at a suitable level of detail. The outputs that are produced by the systems in this research are not intended for interpretation as final designs. Rather, they are a means of exploring the generation of architectural content, elements, and environments with large amounts of raw detail.

Concerning content this research focuses on the production of parametric and procedural systems to generate outputs for further detailing, application, and utilisation by other parties. However, more often than not these systems are laid out in ways that appear illogical, either as a result of multiple revisions and modifications or otherwise. Daniel Davis (2013) addressed this, writing:

"[To] design using [...] functions necessitates that designers have some notion of the design outcome prior to modelling. This upfront planning can be challenging, particularly in a process as notoriously hard to anticipate as the design process" (p. 40).

As a result, it is virtually impossible for anyone except the designer to make modifications to the system. Hence, optimisation and user-friendliness become important characteristics to consider in any system.

Construction of any generative system also requires time. Time in this sense relates to both the development and operating time of any given system. This research demonstrates that virtually any architectural geometry could arise from generative means, but there are many situations where individually modelled elements inserted as block references could achieve more successful outcomes, and faster. However, this is largely dependent on the user, their requirements, and their goals. As with any program, Grasshopper outputs are only as good as the instructions given.

Architectural visualisations also fall outside the scope of this research. Visualisations are a powerful tool in architecture, as in many other industries (Bryant, 2013). However, their use places the final image above all else, ignoring any implications, limitations, and developmental work (Quirk, 2013). By displaying all of the work in the same raw aesthetic comparisons can be made directly on the outputs, rather than the quality of any visualisation.

\section{METHODOLOGY}

This research employed two methodologies. The first is reverse engineering, which involves the deconstruction of existing architectural geometries, and attempting to recreate them within a visual programming environment. Each geometry presents a new or different challenge and a multitude of ways to achieve a resolution. Identifying the most appropriate solution(s) requires familiarity and understanding of the available tools, knowledge of how to implement them, and a willingness to try previously untested ideas.

The second methodology of this research is iterative design. Each system, new or otherwise, has been developed through a series of iterative design stages, including:

\author{
Preliminary: \\ - Drawn interpretation \\ - Planning \\ - Drafting of rules required
}

Sketch:

- Establish parameters

- Create first build in Grasshopper

- Identify and resolve issues where possible

\section{Developed:}

- Resolve any outstanding, or new, issues

- Experiment with different solutions

- Optimise to improve processing time and operating efficiency

\section{Final:}

- Refinement of the developed system

- Reduction of visual clutter

- Improvement of the visual outputs 


\section{PROCEDURAL MODELLING}

"Procedural modelling deals with (semi-)automatic content generation by means of a program or procedure. Among other advantages, its data compression and the potential to generate a large variety of detailed content with reduced human intervention, have made procedural modelling attractive for creating virtual environments increasingly used in movies, games and simulations." (Smelik et al., 2014)

The application of procedural modelling techniques allows for the production of detailed architectural models for game and film environments, at lower costs (Mueller et al., 2006). However, the skills required for these roles are typically in more specific software packages than Rhino/Grasshopper. Researchers are well aware that these teams cannot explicitly model and articulate large scale digital environments to the ever increasing level of detail, or quantity demanded, especially while working with shorter timeframes, tighter budgets, and consistently growing competition (AlJamea, 2017). Rob Hoffman (2007) wrote:

"II]t is clear that audience demand for 3D content is growing rapidly. For example, of the top 50 grossing films of all time globally, over three-quarters are either all-CG or heavily dependent on computer-generated visual effects." (para. 3)

Michael James (2015) also wrote:

"Because of the damage done by [Computer Generated Imagery (CGI)], Hollywood can only finance CGI-fest films with bloated budgets. The people demand CGI and the only way to keep the demand up is to increase the dose of CGl." (para. 8)

As a result of this requirement, the area of procedural modelling has been an active area of research for over 30 years, specifically in the field of city generation (Smelik et al., 2014). These researchers have developed many of the practices and programs that are commonplace today (Bazalo \& Moleta, 2015; Welch, Moleta \& Moloney 2014)

\section{OPTIMISATION}

Any given problem is approachable in a multitude of different ways. There are often good and bad solutions, both objectively and quantifiably. Even something as straightforward as creating a cube can be completed in many different ways, depending on the selected approach. On this matter Addison Duvall (2016) wrote:

"No matter what you want to accomplish in a design, there is almost always a simpler way to do it that will obtain the same result with less distraction and clutter."

Unsurprisingly, a considerable portion of this research has involved the optimisation of systems. With regards to Grasshopper, these optimisations allow improvements to accessibility, modifiability, reusability, and processing time. When generating basic systems none of these factors is a concern. However, in more extensive systems seemingly insignificant inefficiencies add up rapidly. This result in increased processing time(s), as well as unexpected and occasionally unresolvable issues.

The degree of visual clutter on a canvas is directly related to its understandability. Any system that poorly managed from an early stage becomes difficult to operate, modify, or reuse at a later date. Poor canvas management is capable of rendering a system completely unusable by its designer, and other parties alike. This is widely known as Spaghetti, and on the matter, Daniel Davis (2010) wrote:

"Often when you are producing spaghetti, everything will be fine, but when it comes to editing or reusing spaghetti [...] it almost impossible to understand what your code does. [...] [Y]ou do not know what removing a node does or how to reconnect the other nodes when you do." (para. 2)

With these factors in consideration, it is necessary to understand a variety of methods to approach resolving any given problem. Process optimisation is a crucial feature of any system. It is a necessary factor to ensure that software crashes are avoided or reduced wherever possible, to make sure that there is no loss or corruption of data. 


\section{A PATTERN LANGUAGE}

There are a large number of authors, researchers, and academics whose work deserves discussion in this section. Notable mentions may include the work of Mark Wigley, Greg Lynn, Peter Wonka, and Patrik Schumacher. However, the work that has been most influential on this research is Christopher Alexander's "A Pattern Language" (1977). As noted by Michael Mehaffy, Alexander initially trained in mathematics and physics (2016). His work was concerned with the processes involved in transforming individual parts into coherent wholes. The content of the work is outlined well by Goodreads (2017), stating:

"More than 250 of the patterns in this language are outlined, each consisting of a problem statement, a discussion of the problem with an illustration, and a solution. As the authors say in their introduction, many of the patterns are archetypal, so deeply rooted in the nature of things that it seems likely that they will be a part of human nature and human action as much in five hundred years as they are today." (para. 3)

The book provides an incredibly thorough investigation into designing from nothing, working through a series of instructions and patterns. It functions in a manner that is comparable to a 'choose your own' adventure novel. For example, an individual may be for designing an entrance way. The book will then direct you to investigate the design of the front porch and the balustrades. Then the front garden, the threshold between public and private spaces, how windows open into the front rooms, so on and so forth. The patterns truly range from designing at a city-wide scale, right down to the placement of furniture in a bedroom, or living space (O'hara, 2016). The text provides a powerful base for those who wish to express their design intent in their communities, and homes, without the assistance of Architects or other design professionals (Buffalo Library, 2017). As shown in figure 1.05 Alexander's writing can be interpreted to inform an all-encompassing range of design outcomes and possibilities.

Because of the nature of this work, it presents the perfect opportunity for investigation by computer scientists, and other researchers. Many teams and individuals have attempted to build explicit rule sets from the writing as the basis of their systems. The work has also been incredibly influential in the work of more architecturally minded researchers. The fact that it is still discussed some 40 years later serves as a testament to its brilliance.

Image Redacted for online submission, see physical submission for image 


\section{SOFTWARE ANALYSIS}

\section{CityEngine:}

Widely used by urban planners and related practitioners this program allows for the creation of 3D city environments from GIS data (Biljecki et al., 2016). As such it allows for the generation of context models as a means for urban planners to present and share their work, as well as test different ideas through similar methods (Esri, 2017).

CityEngine encourages a design by numbers approach but also allows for the implementation and control of block elements, as well as control over specific output elements (CityEngineTV, 2016). While this software is incredibly powerful, it would have served as a poor starting point for this investigation. CityEngine has seen a high degree of use in research projects and advertising but has not been used extensively for Film/Game environments (Esri, 2017). While the program allows for the creation of custom environments, its true strength appears to be in generating content from existing data.

\section{Maya:}

As written by Edulearn (2014), Maya:

"Maya is the de facto standard for 3D visual effects, computer graphics, and character animation." (para. 2)

Maya allows for the use of its embedded language, as well as Python scripting, to generate customised commands and actions. Basic city generation tools provide a simple starting point for contextual models and further articulation. When city environments have formed, it is often through the implementation of Python within Maya, and a reliance on basic subdivisions. Daniel Peter (2014) suggests that this process is more useful for rapidly generating specific structures, rather than a procedural exploration.

An advantage of working in Maya over Grasshopper would be explicit model controls. Grasshopper works best when geometries remain live and unbaked, but the process of articulating any idea can be a lengthy process. Transferring from one modelling space to another is not a concern in Maya as the geometries exist in the modelling space from their conception. Furthermore, because Maya has been around for some time, a large number of plugins have been crafted to add new and different capabilities (Tabarsi, 2016).

\section{Python Scripting}

Zybrand Jacobs states that:

"Python is [...] the best general purpose code to learn because it's used everywhere" (Tabarsi, 2016, para. 5).

Python provides the base of Random3DCity and has been used within Grasshopper by a group of students in Sydney for similar purposes to this body of research. Through Python, Random3DCity is capable of generating a range of different LoD outcomes for city environments and proposes a means of standardising and adding to the CityGML catalogue (Biljecki et al., 2016).

Students at Sydney University utilised Python in a different way, to custom script components within Grasshopper (Coorey, 2017). This application is incredibly powerful and presents the opportunity to create incredible work, while still harnessing the best elements of the base program. Scripting, in general, is incredibly powerful in this respect and allows for far greater control of Grasshopper.

\section{Houdini:}

Houdini, like Maya, is considered industry standard software. As a program, it is incredibly powerful. Houdini employs a procedural workflow, combined with a visual programming interface for reasons explained on their website:

"[A]ll the steps needed to set up a shot are represented by networks of nodes which are maintained deep into production [of a project]. This makes it easy to make last-minute creative decisions that would be too costly in a traditional CG pipeline" (SideFX, 2017, para. 3).

The program also acts as a complete platform, including the ability to model, complete physics simulations, character-rigging for animation, rendering, and so on. Because of its node-based system it also allows for, and retains, a far greater degree of control than many other packages. However, Houdini is considered significantly harder to learn than other more singularly focused design tools (Kluyskens, 2016). While it is extremely powerful software, it would be underutilised in this research. 


\section{CHAPTER TWO \\ PRELIMINARY DESIGN}




\section{PRELIMINARY DESIGN}

The investigation of the golden ratio involved reverse engineering an existing geometry through the use of a proportionately defined system. One downside to the utilisation of a proportionate system is the larger number of mathematical operations involved, which translates into a longer construction time. If mismanaged this can result in increased visual clutter on the Grasshopper canvas, making the entire system difficult to reuse or modify at a later stage (Davis, 2010).

As an explicit modelling system, this tool would only work for a specific set of dimensions. A proportionally defined system is more flexible, requiring less user intervention once constructed. However, it requires a greater deal of understanding of the system's logic and the various elements involved before construction begins.

In this situation, anytime a rectangular form was drawn Grasshopper would be deconstructed the geometry and reform it as a golden section, as shown in Figure 2.01. This action takes place regardless of the rectangles original dimensions, proportions, or location. Furthermore, the operation takes place in a user specified direction, using the rectangle's $X$ or $Y$ values to inform the size of the overall golden section. This process operated through a series of logic gates built into the system, which managed the data distribution throughout the Grasshopper canvas.

Figure 2.01: (Top) Single section output Figure 2.02: (Middle) Single \& Rotated section Figure 2.03: (Bottom) Single, Rotated \& Mirrored sections
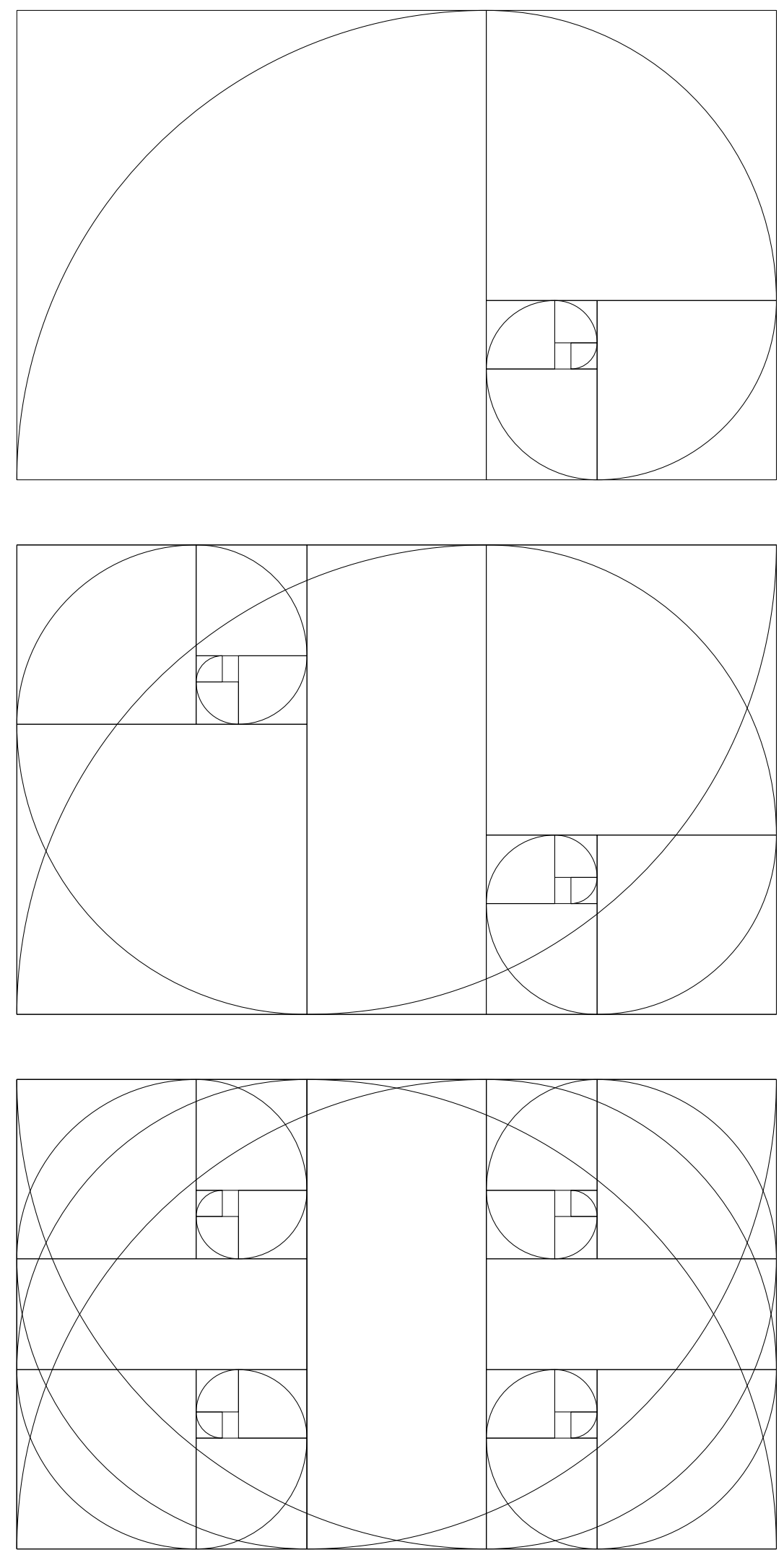


\section{VILLA CAPRA “LA ROTONDA"}

Following the success of the first investigation, attempts to recreate a proportional three-dimensional geometry began. While investigating the golden section its prevalence in Andrea Palladio's architectural works became apparent (Fletcher, 2000). One of the best examples of this was Villa Capra, constructed in 1566 (Díaz, 2013). Villa Capra has been analysed and deconstructed by innumerable people, providing a strong starting point for the recreation of the built form.

As the building positioned itself as the first of many built forms to be created in Grasshopper, it was logical to develop systems for individual architectural components for installation in future works. It is initially more time consuming to construct a system rather than explicitly model the form, but the resultant system becomes infinitely more reusable than any modelled geometry. Unfortunately, explicitly modelled geometry is only truly usable in its initial setting. Unlike the systems developed in this research, these geometries need to be heavily modified, or completely rebuilt, to be utilised in a new modelling situation.

Image Redacted for online submission, see physical submission for image 


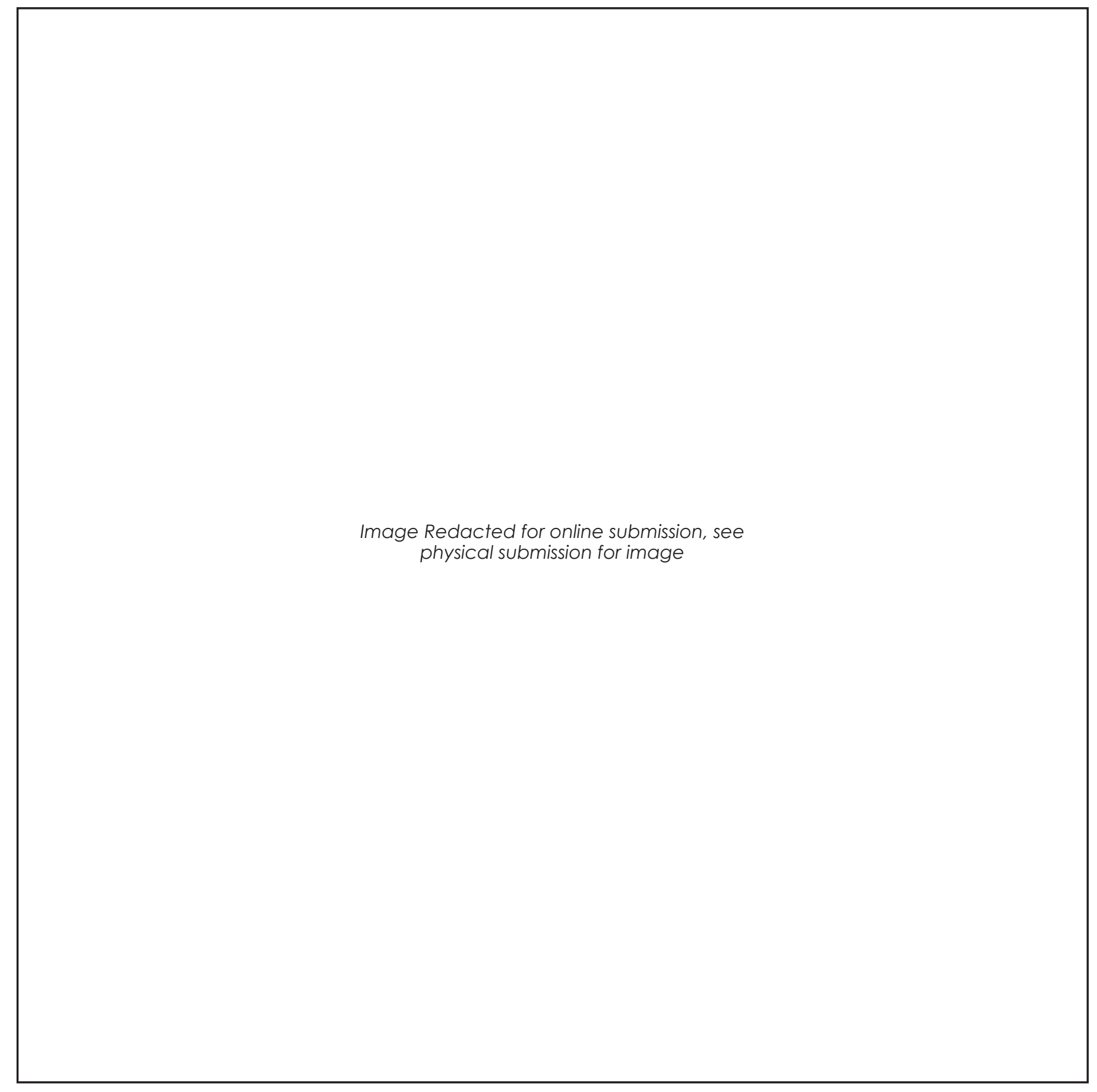

Figure 2.05 / 2.06: Palladio's Villa Capra Side / Front (Baver, 2007)

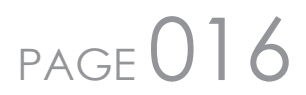



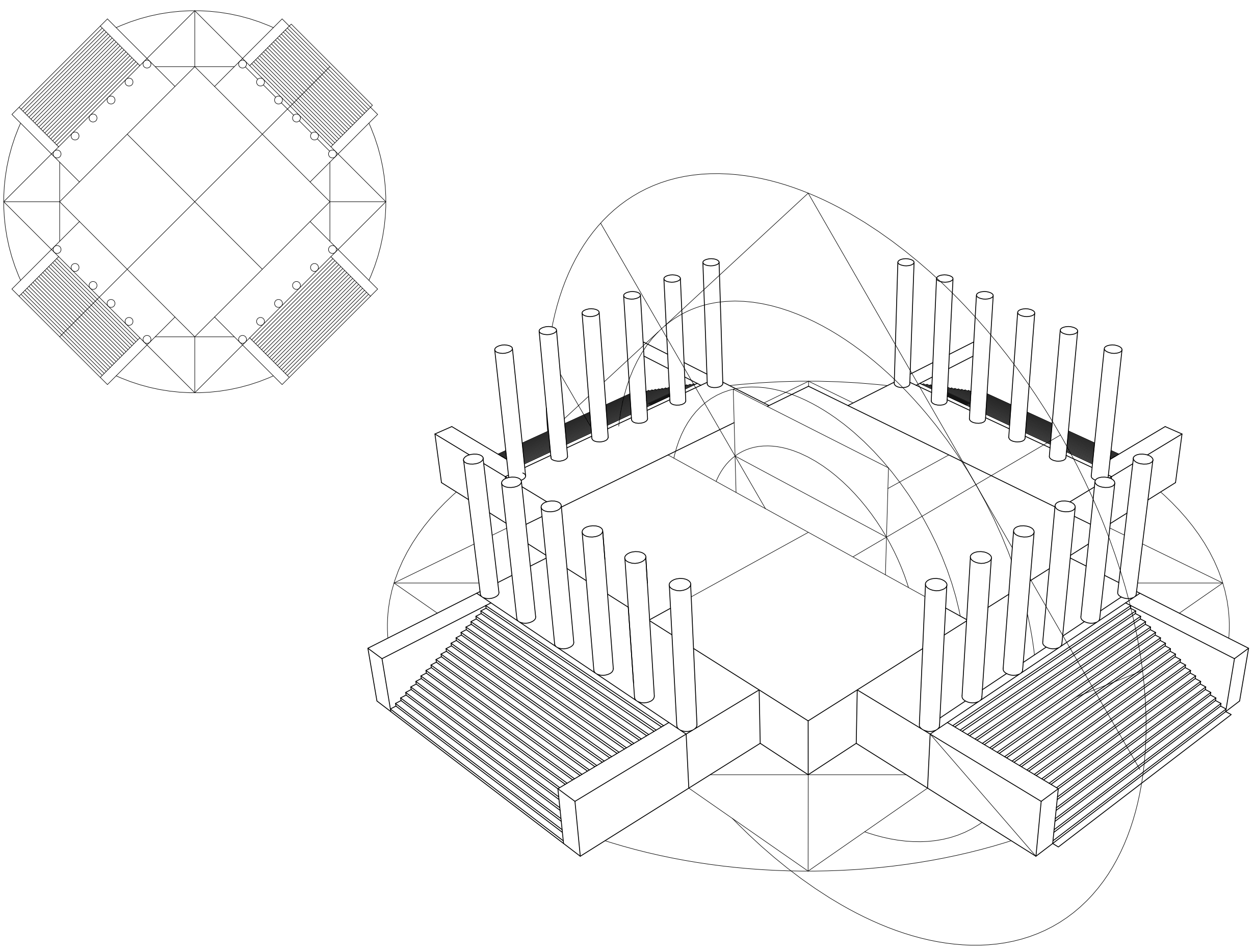

Figure 2.07: Plan \& Perspective line drawings of Grasshopper output version 01 

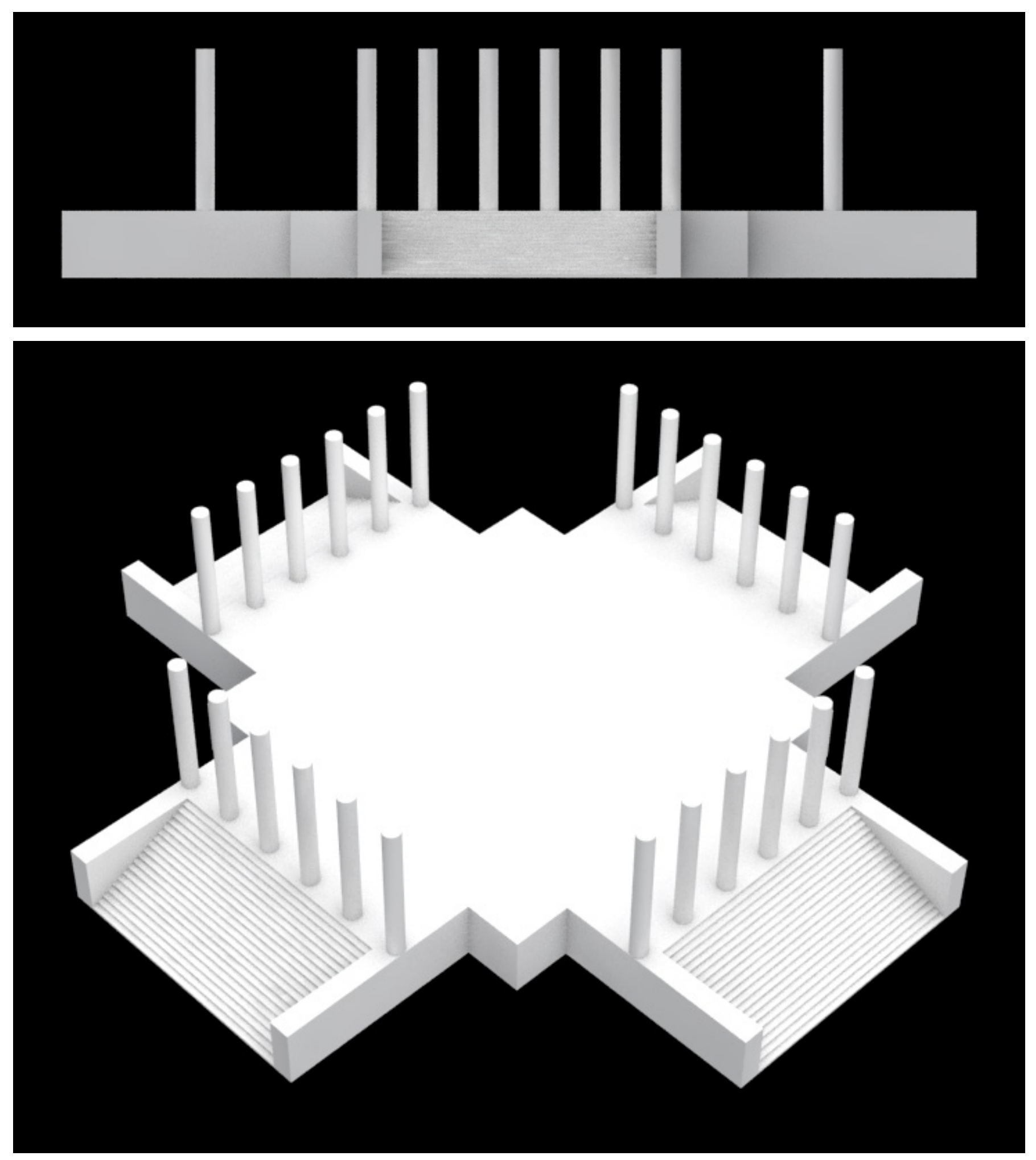

Figure 2.08: Elevation \& Perspective clay renders of output 01 


\section{PARAMETRIC REVERSE ENGINEERING 02}

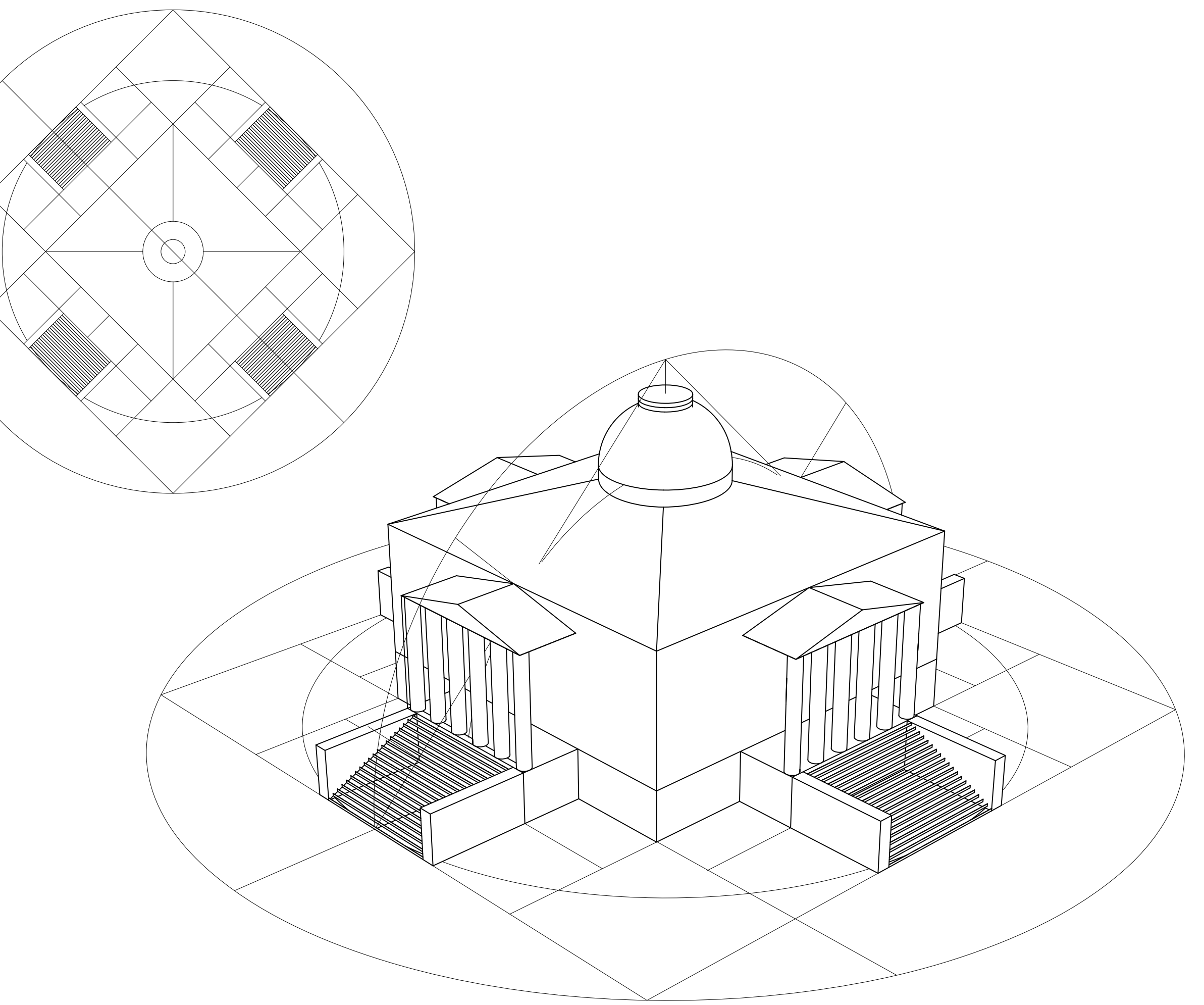

Figure 2.09: Plan \& Perspective line drawings of output 02

CHAPTER TWO 

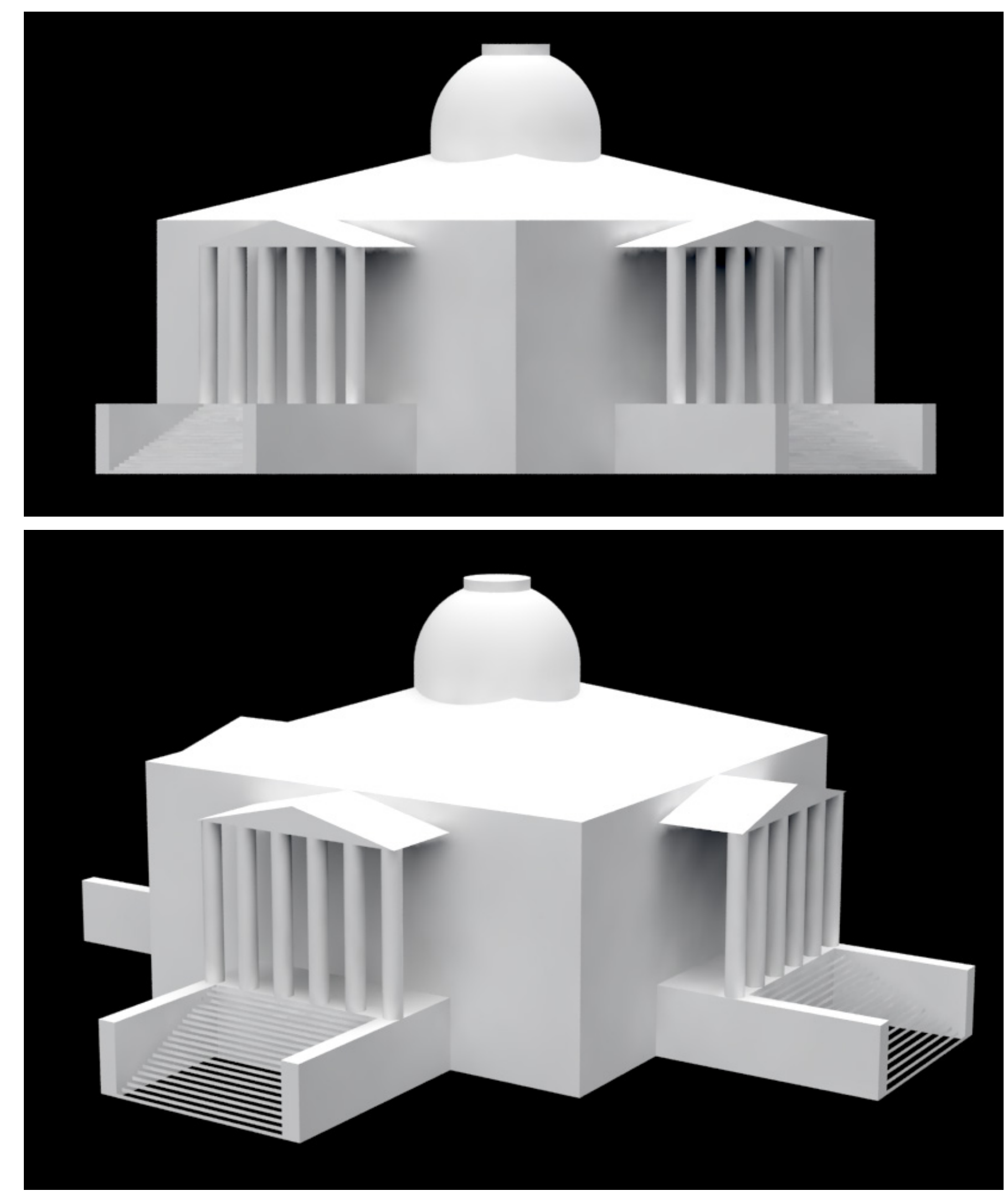

Figure 2. 10: Elevation \& Perspective clay renders of output 02 


\section{COLUMN GENERATOR}

The first column system constructed involved the analysis and recreation of classical column geometries. This investigation was undertaken parametrically because of its intended application in the construction of future tools. The alternative would have been to explicitly model only the necessary column forms, for use in a single modelled iteration. The columns, as a built element, were selected for many of the same reasons as Villa Capra. Because of their prominence in the Villa, it was logical to deal with them first.

Classical columns have been heavily analysed, meaning that the rule sets for their creation are clearly defined. Columns of the classical orders are controlled by various rules, ratios, and so forth.

Columns presented a suitable choice for an intensive geometric exploration because of the implications and understandings achievable having constructed them, and their prevalence in the Villa. If a column can be defined in a parametric or procedural manner then so too can any other number of built elements. These forms may include but are in no way limited
- Windows
- Doors
- Stairs / Railings
- Roofs
- Room layouts
- Green space

Because of these implications, it was deemed unnecessary to investigate to investigate alternative small-scale geometries. 


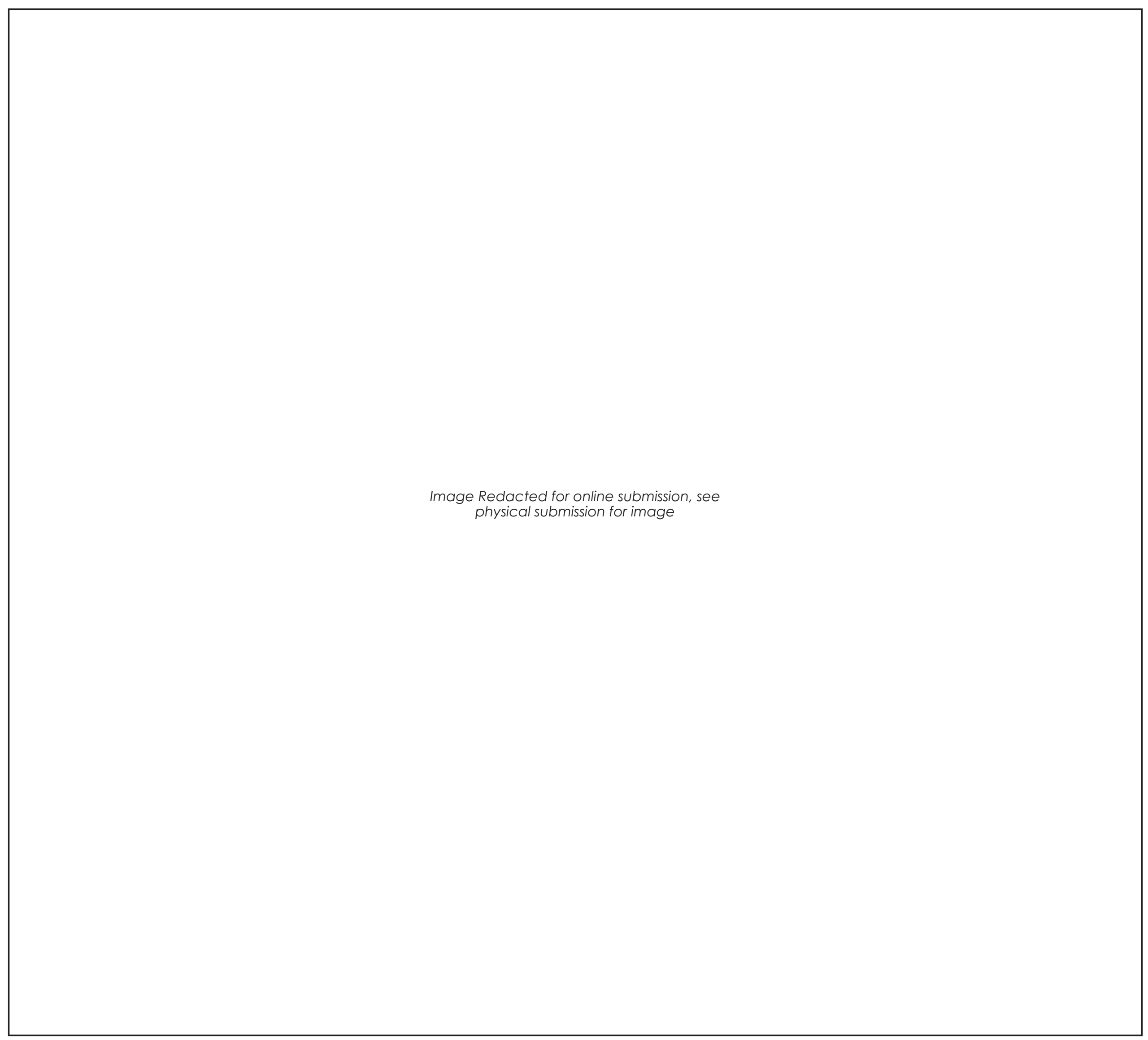

Figure 2.11: Analysis diagram of Classical Column orders (Rouchell, 2013) PAgE 022 


\section{GRASSHOPPER COLUMN OUTPUTS}

Tuscan

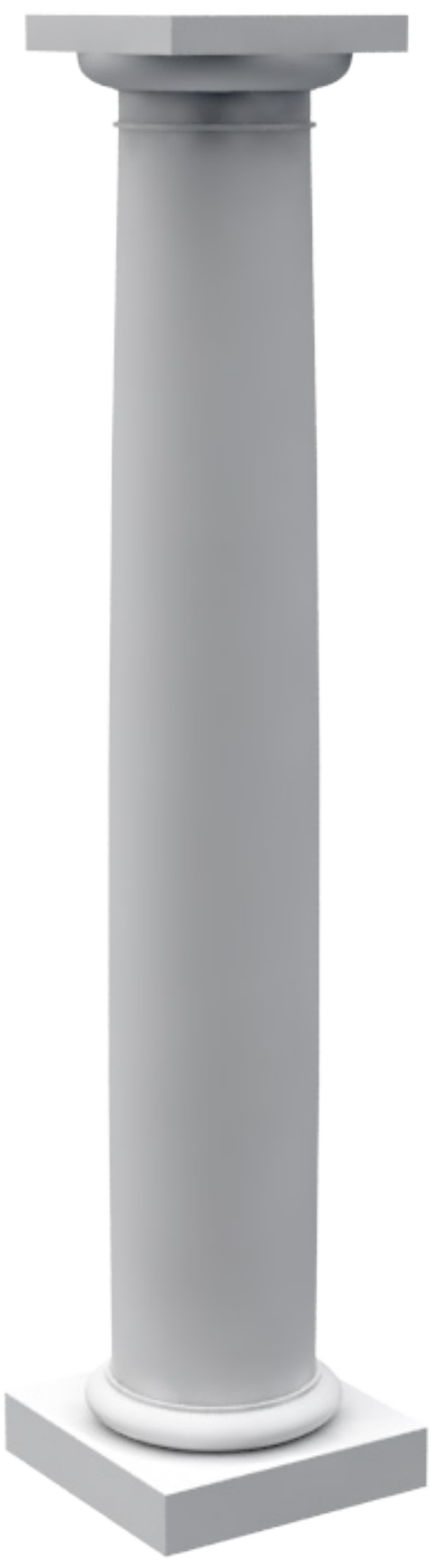

Doric

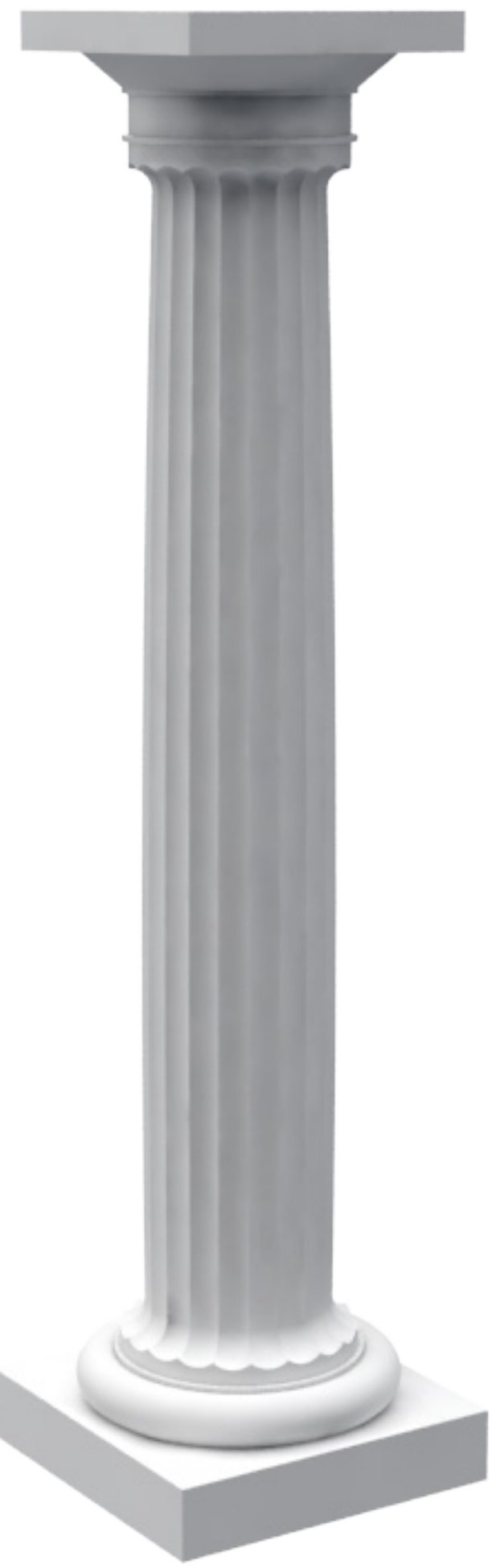

Ionic

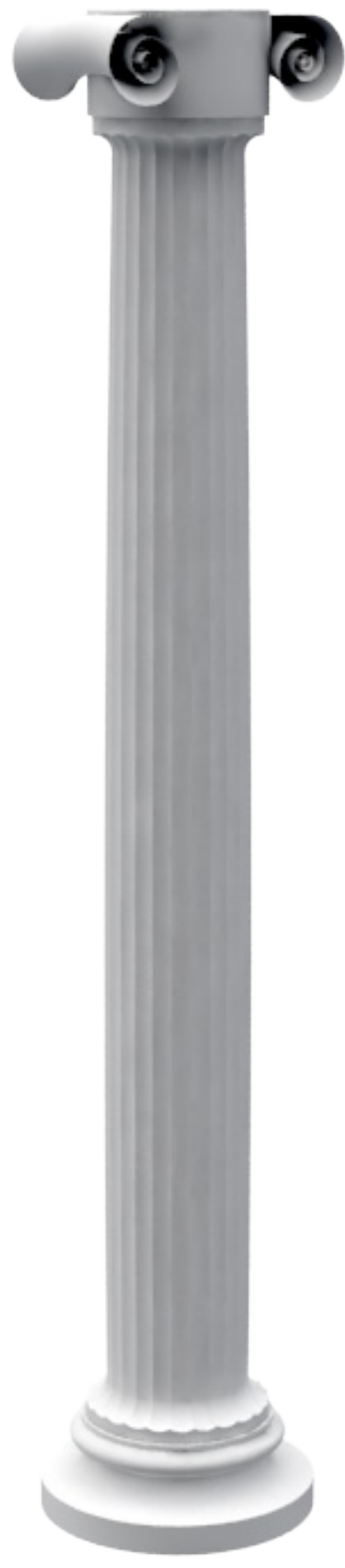

Corinthian

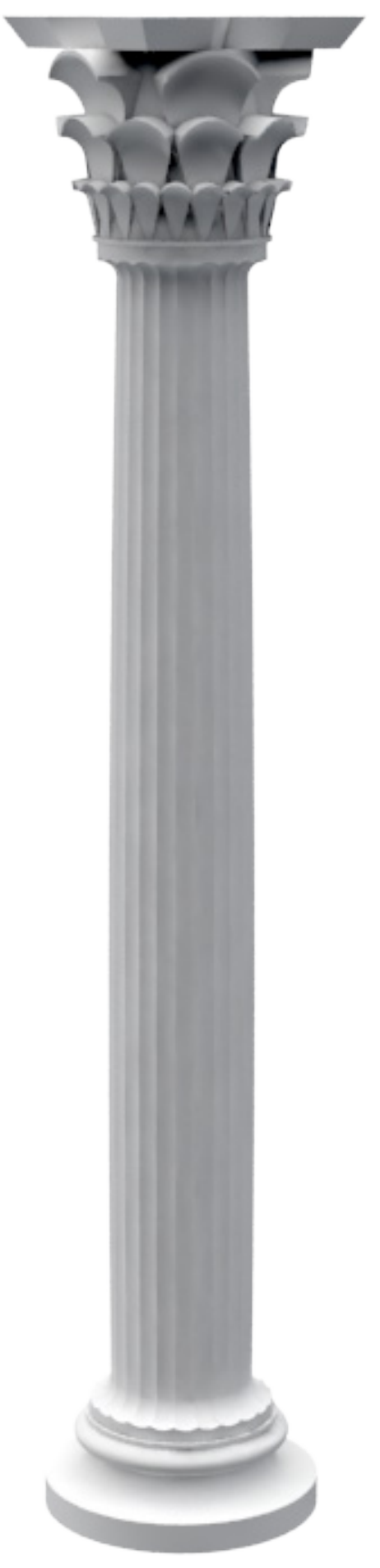




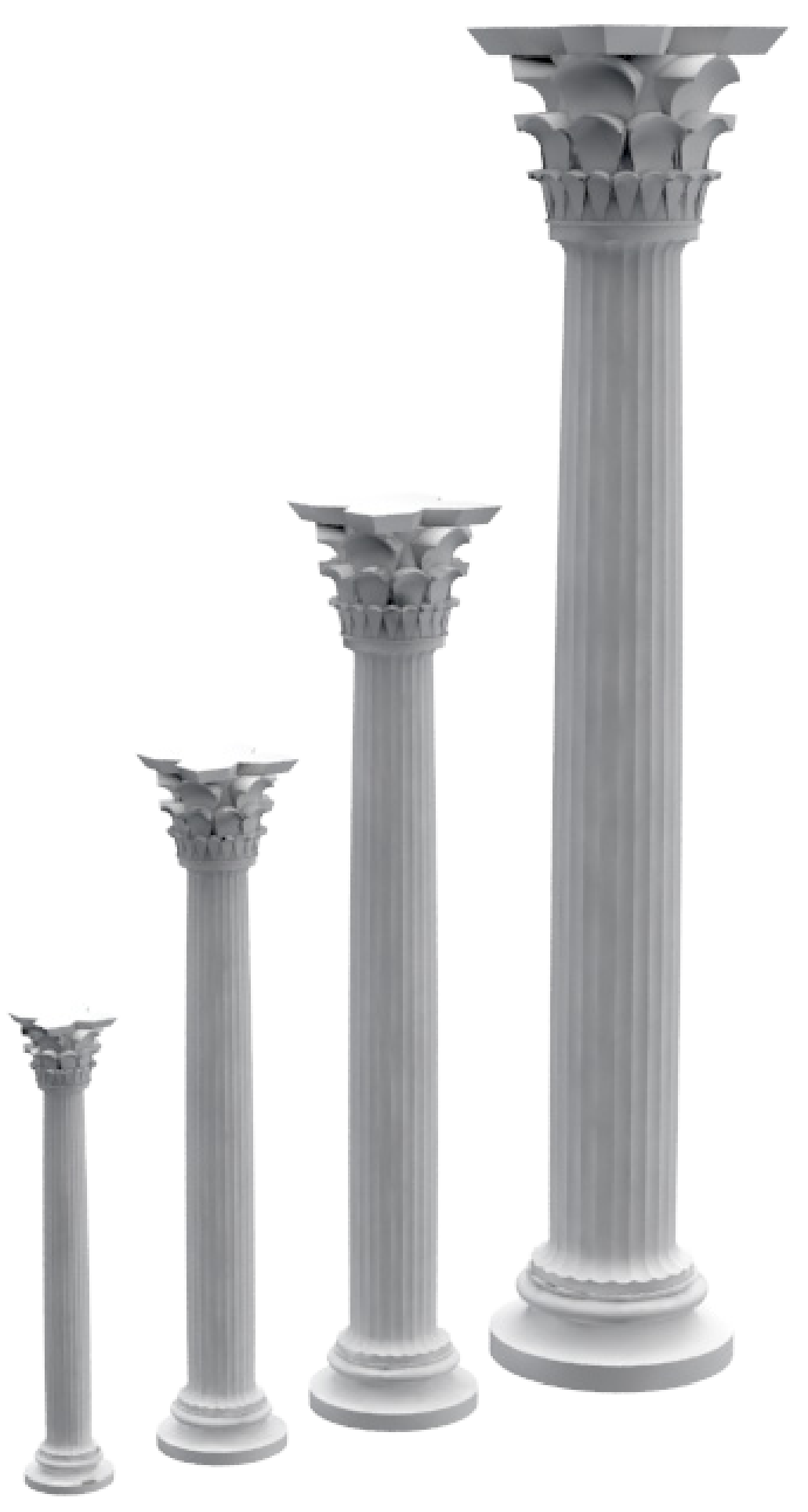

Figure 2.13: Composition of proportionate generation of Corinthian columns

$$
\text { PAgE } 024
$$




\section{COLUMN GENERATOR ISSUES}

To parametrically define the natural forms, such as those found on the Corinthian column, was incredibly complex. An alternative considered at this point was the utilisation of block references, through the use of various plugins. However, this ventured too far towards an explicit modelling exercise.

In this system logic gates were established to select which of the four column types would be generated, based on a generated value between 0 and 1 :

\begin{tabular}{|c|c|c|c|}
\hline Tuscan & Doric & Ionic & Corinthian \\
\hline $0-0.25$ & $0.26-0.50$ & $0.51-0.75$ & $0.76-1.00$ \\
\hline
\end{tabular}

This same seed value previously dispatched as scaling for the circles was also used to form the fluting of the column's shafts, impacting the lonic and Corinthian significantly. Because the Corinthian had the highest value range it experienced the highest degree of scaling, and as such looked impossibly thin. Unexpected and seemingly minor errors such as these can have large impacts on the outputs of any system.

Spheres were also used to create the base and top of the column shafts. Once generated, the spheres subtracted solid volumes from a base cylinder. When combined with the scaling issue, this resulted in output times exceeding of 15 seconds, as each sphere was interacting with virtually every other. The solution to was significantly less three dimensional. Generating similar results in 2D generated outputs significantly faster, with an unnoticeable difference.
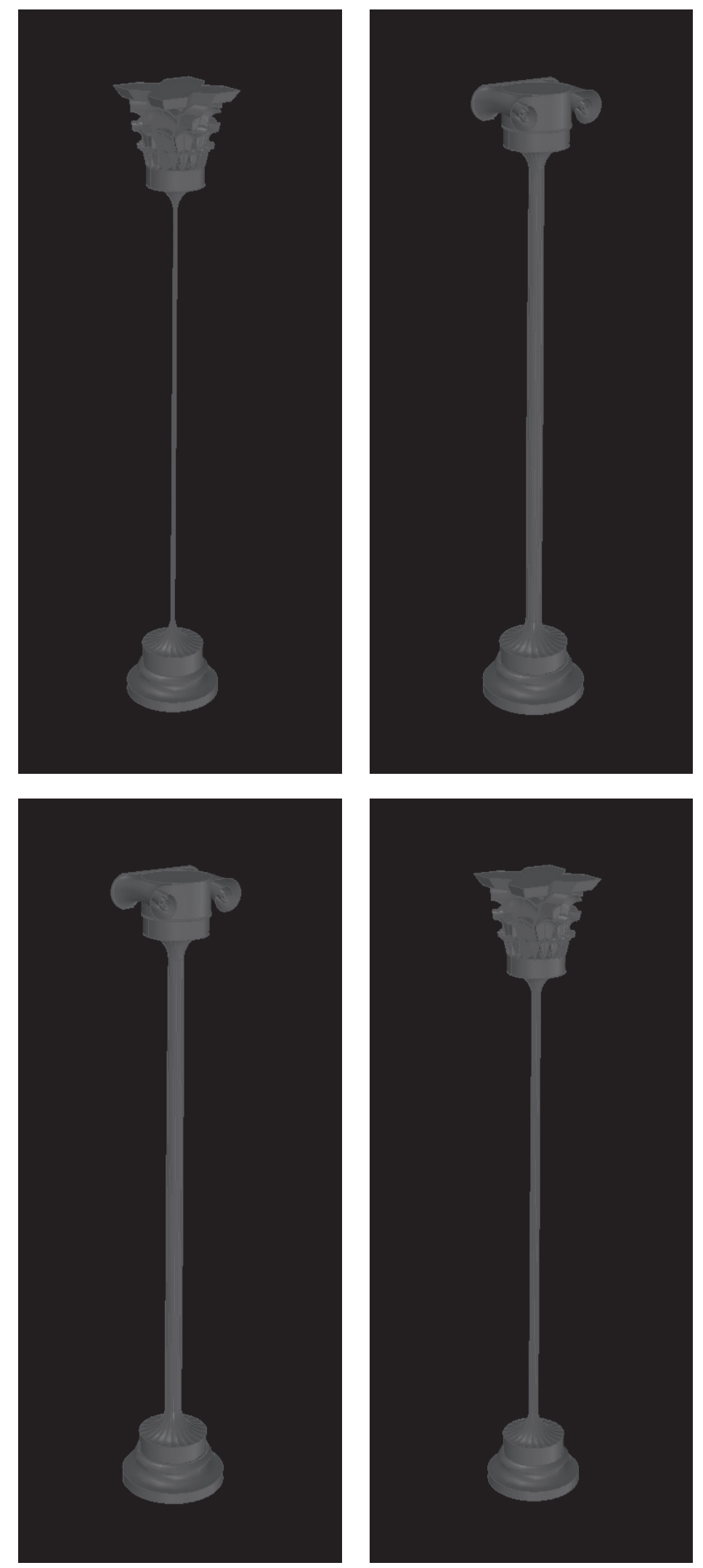

Figure 2.14: Failed procedural lonic \& Corinthian column outputs 
SEED VALUES \& RANDOMISATION

To properly investigate each system's outputs a seed generator was deemed necessary. Seed values, as David Rutten (2012) wrote on the Grasshopper forum, are:

\begin{abstract}
"[l]ntegers that define the exact sequence of pseudo-random numbers [...]. Even the tiniest change in seed value will result in a radically different random sequence. (para. 11)
\end{abstract}

[What are the] characteristics that define pseudo-randomness?

- The sequence of generated numbers should never repeat itself.

- The numbers in the sequence ought to be spread evenly across the numeric domain." (para. 4)

Each seed value is unique and will consistently produce the same set of values, regardless of its situation. Table 1 and 2 explore this. As long as an effort is made to document the unique or interesting seed values, then there is no need to export each unique Grasshopper output. Instead, a documented seed number can be reinstalled at a later date to generate the same geometry, provided the system was not modified.

In order to explore a range of options rapidly, seed generators dispatched values at multiple stages throughout this research. The system makes use of the current date and time to produce ever-changing sets of numbers, as displayed in Table 3. This generated seed value is then fed into a random number component, producing results shown in Table 4.

While this system does not solve every issue, it is a far better option than using the same set of seed values consistently. If systems do not utilise different number sets throughout the design process, then the creator may be under the false impression everything works perfectly, always.
Table 1: Different Range, same seed value:

\begin{tabular}{|c|c|c|c|c|c|c|c|c|c|c|}
\hline Range & 1 & 2 & 3 & 4 & 5 & 6 & 7 & 8 & 9 & 10 \\
\hline $1-100$ & 86 & 70 & 66 & 68 & 28 & 94 & 64 & 41 & 24 & 12 \\
\hline $1-1,000$ & 854 & 700 & 661 & 683 & 278 & 932 & 644 & 412 & 239 & 124 \\
\hline $1-10,000$ & 8541 & 6996 & 6606 & 6824 & 2783 & 9321 & 6434 & 4125 & 2393 & 1238 \\
\hline
\end{tabular}

Table 2: Same range, different seed values:

\begin{tabular}{|c|c|c|c|c|c|c|c|c|c|c|}
\hline $\begin{array}{c}\text { Seed } \\
\text { Value }\end{array}$ & $\mathbf{1}$ & $\mathbf{2}$ & $\mathbf{3}$ & $\mathbf{4}$ & $\mathbf{5}$ & $\mathbf{6}$ & $\mathbf{7}$ & $\mathbf{8}$ & 9 & 10 \\
\hline 101 & 1 & 43 & 39 & 47 & 66 & 97 & 39 & 93 & 58 & 35 \\
\hline 102 & 68 & 26 & 22 & 29 & 49 & 80 & 22 & 76 & 41 & 18 \\
\hline 103 & 34 & 9 & 5 & 12 & 32 & 63 & 5 & 59 & 24 & 1 \\
\hline
\end{tabular}

Iable 3: Same day / different times

\begin{tabular}{|c|c|c|c|}
\hline Date & Time & (Date) $*$ (Month) $*$ (Year) * (Hour) * (Minute) $*$ (Second) & Seed Value (Result) \\
\hline $\mathbf{0 9} / \mathbf{0 2} / \mathbf{2 0 1 7}$ & $13: 24: 30$ & $9 * 2 * 2017 * 13 * 24 * 30$ & 33982416 \\
\hline $\mathbf{0 9} / \mathbf{0 2} / \mathbf{2 0 1 7}$ & $13: 24: 35$ & $9 * 2 * 2017 * 13 * 24 * 35$ & 396461520 \\
\hline $\mathbf{0 9} / \mathbf{0 2} / \mathbf{2 0 1 7}$ & $13: 24: 40$ & $9 * 2 * 2017 * 13 * 24 * 40$ & 453098880 \\
\hline
\end{tabular}

Table 4: Resultant seed value numeric outputs:

\begin{tabular}{|c|c|c|c|c|c|c|c|c|c|c|}
\hline Seed Value & 1 & $\mathbf{2}$ & $\mathbf{3}$ & $\mathbf{4}$ & $\mathbf{5}$ & $\mathbf{6}$ & $\mathbf{7}$ & $\mathbf{8}$ & 9 & 10 \\
\hline 339824160 & 5 & 26 & 54 & 91 & 35 & 87 & 48 & 17 & 93 & 77 \\
\hline 396461520 & 99 & 20 & 48 & 85 & 30 & 82 & 42 & 11 & 87 & 72 \\
\hline 453098880 & 61 & 82 & 10 & 47 & 92 & 45 & 5 & 73 & 50 & 34 \\
\hline
\end{tabular}




\section{ERRORS}

Parametric processes allow designers to explore scores of iterations rapidly. This large-scale analysis can aid the identification of issues within the built system and raises questions regarding the acceptability of errors. This research argues that it is entirely situational. Concerning errors these situations may include:

- Complete error ( $100 \%$ fail rate): Restart. Identify the issue and find a new solution

- High-frequency errors (50\%): Must be fixed, or this will have a significant impact the system operation and outputs. Likely reflects a major issue in the Grasshopper logic

- Medium-frequency errors (10\%):

Can be ignored. A large number of the system's outputs will have visible flaws, detracting from the larger series. Suggests a small issue in the Grasshopper logic

- Low-frequency errors (1\%):

Can be ignored as the chance of encountering these problems is low. Fixing these issues can be incredibly time-consuming, with little reward. If a system must operate with $100 \%$ reliability, then these must also be eliminated

\section{PROCEDURAL INVESTIGATION}

A growing number of video game developers are utilising Procedural Generation techniques to producing significant amounts of content with relatively small teams, and budgets. Popular games of this genre include Minecraft and Spelunky, while large series such as Civilization, and Borderlands, make use of procedural generation to add to their core gameplay experience (Baker, 2016). The game No Man's Sky relies entirely on procedural generation, and with a team of just 11 people were able to create planets, vehicles, buildings, creatures, weapons, and more (Hello Games, 2016).

- Planets: Hello Games have created a universe containing 18 quintillion different planets (Hussain, 2016). However, the planets as a whole suffered from a common criticism of procedural works, in that after a while the outputs all began to appear similar

- Creatures: A large number of creatures encountered by players appeared completely ridiculous, unbelievable, and poorly constructed, which detracted from the game as a whole

- Vehicles / Buildings: Vehicles and buildings had an incredibly large degree of variation, added onto a small number of base frames. The result was controlled, consistent, and interesting outcomes. It was very apparent that this system used more reference geometry than pure procedural generation, and had fewer variables to contend with

Elements of this system are incredibly successful. However, this was one of the first 'big' releases to rely almost entirely on the procedural generation for content. Unfortunately, No Man's Sky will likely taint the idea of future procedural game development because of its assumption that procedural content alone would make for an interesting gameplay experience. 
Image Redacted for online submission, see physical submission for image 


\section{PROCEDURAL COLUMNS}

As a means of exploring procedural generation techniques in an architectural context, the parameter ranges used to define the modelled elements of the classical column's shafts were opened up. Making this modification allowed for the resultant columns to become something different, and unexpected.

The column shafts were allowed an incredibly wide range of values for rotation, scaling, fluting, amongst others. These were developed to demonstrate and gain an understanding of the potential of such a system, rather than to create real-world geometries. Through this approach, a variety of outputs were able to be produced with limited intervention, bias, or time investment beyond creating the initial system.

The outcomes generated by the system have merit, but many appear as primarily scaled up, rotated versions of the original column forms.

TUSCAN

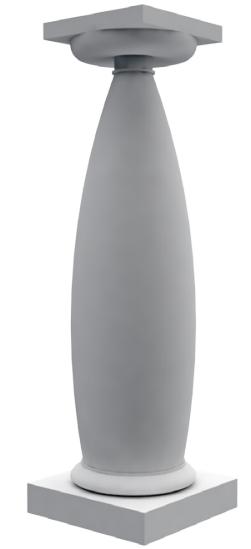

Output 01

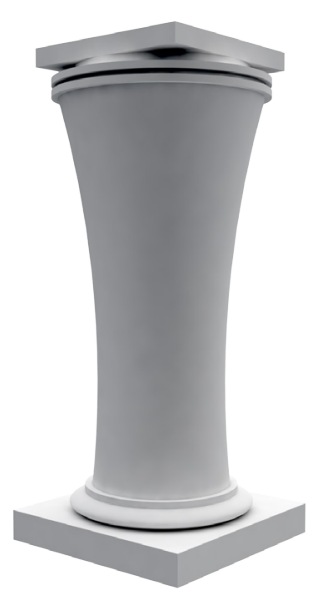

03

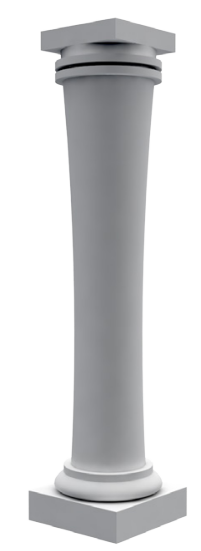

05

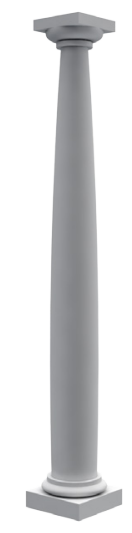

02

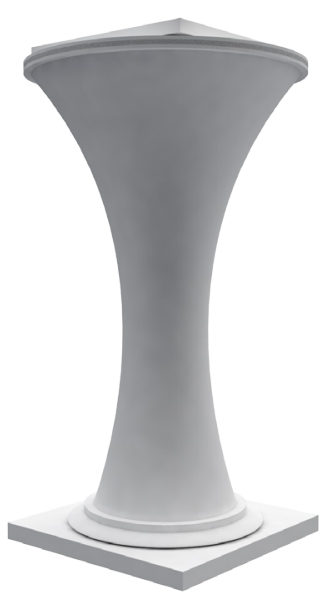

04

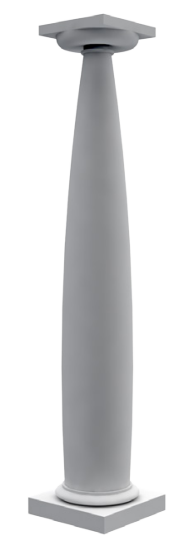

06 


\section{DORIC}

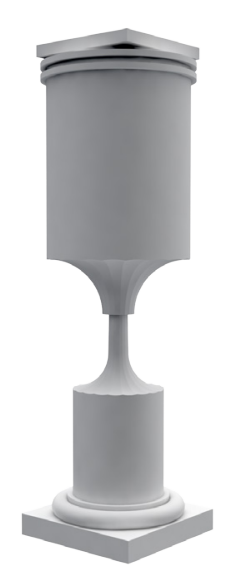

Output 01

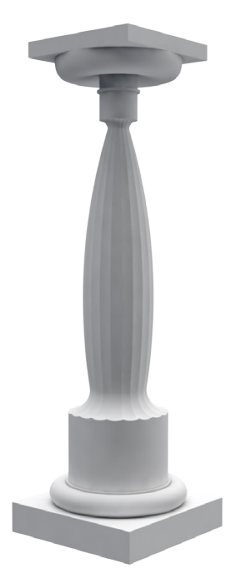

06

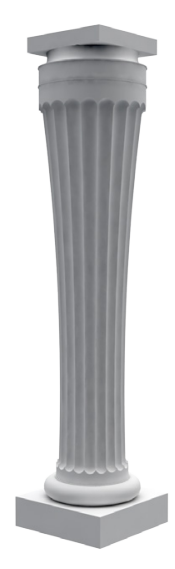

11

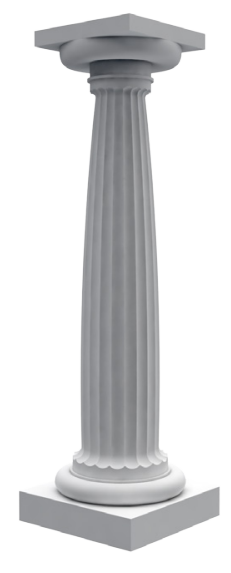

02

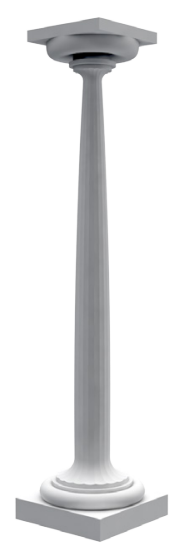

07

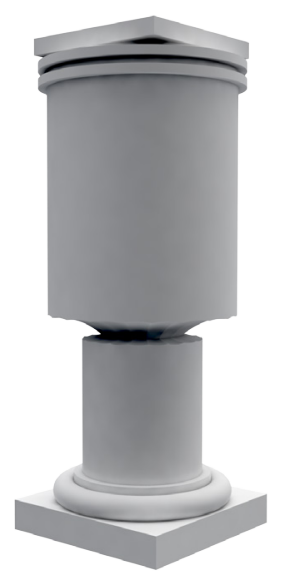

12

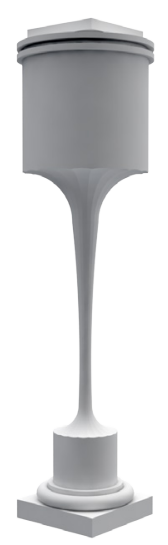

03

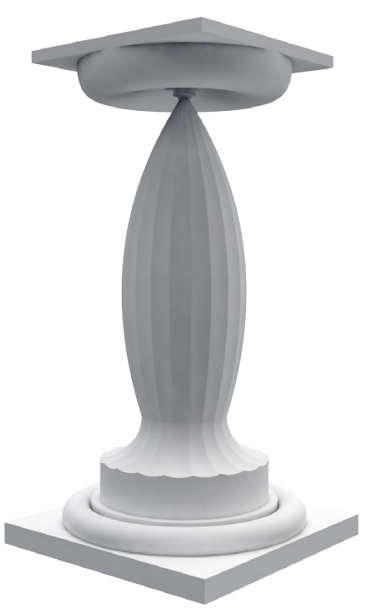

08

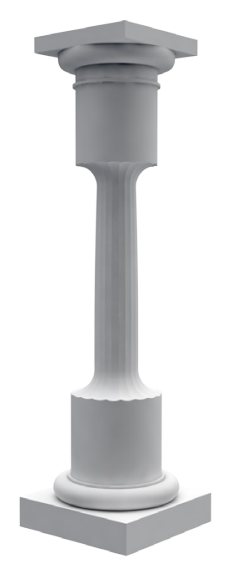

13

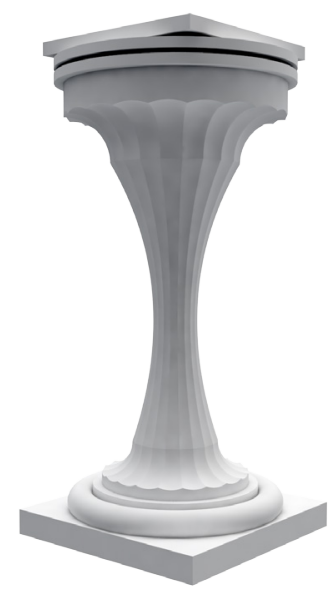

04

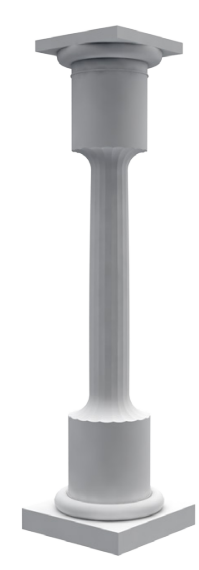

09

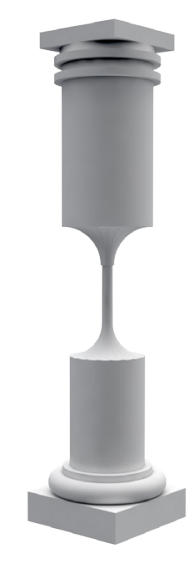

14

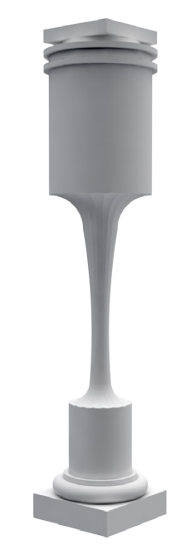

05

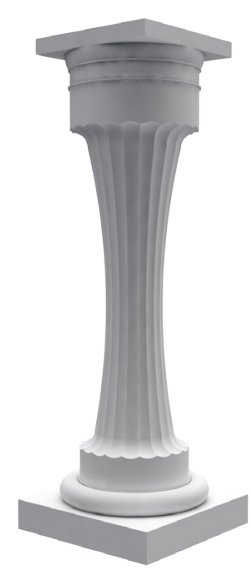

10

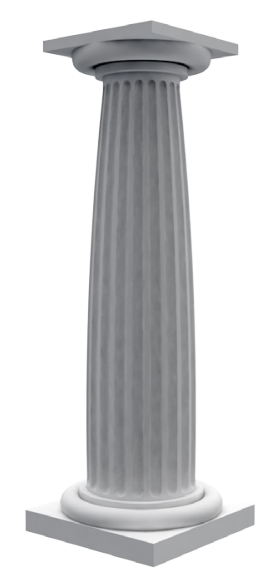

15

Figure 2. 18: Composition of procedurally generated Doric columns 


\section{IONIC}

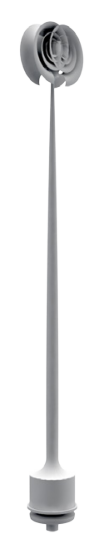

Output 01

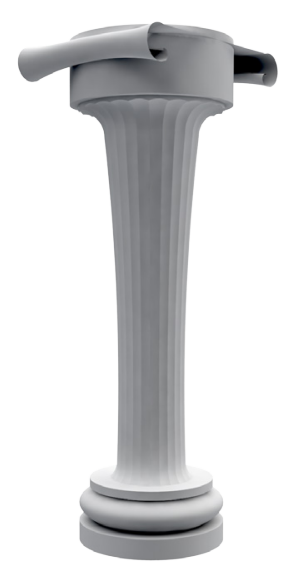

06

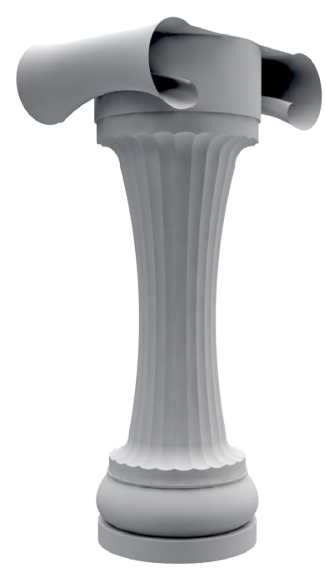

11

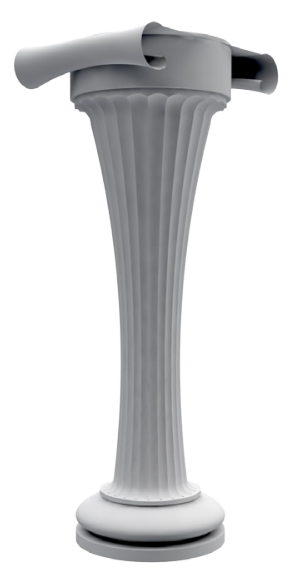

02

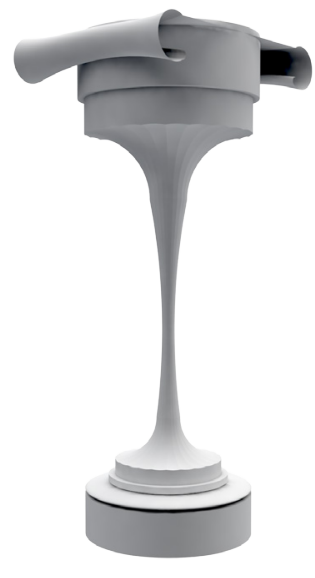

07

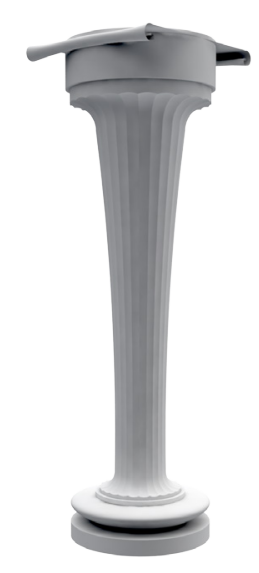

12

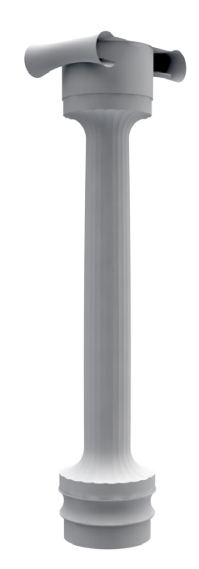

03

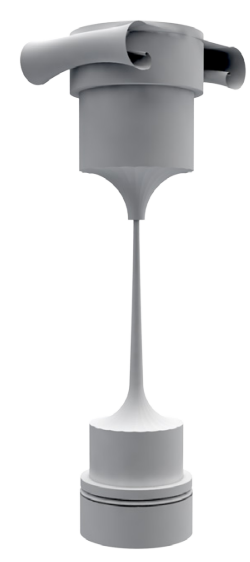

08

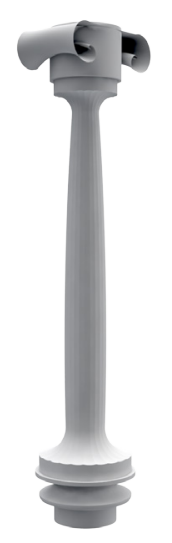

13

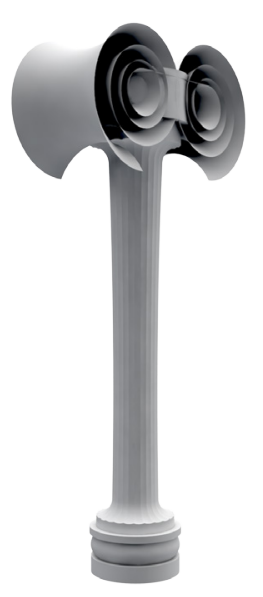

04

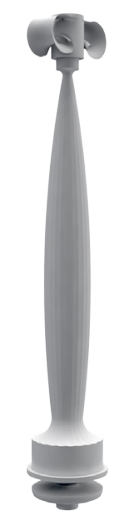

09

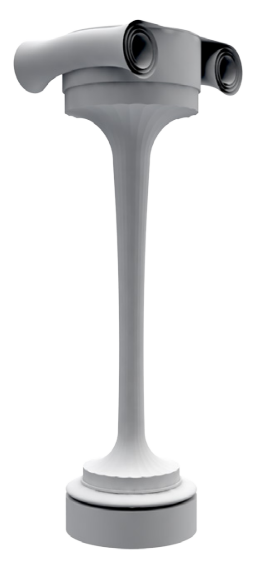

14

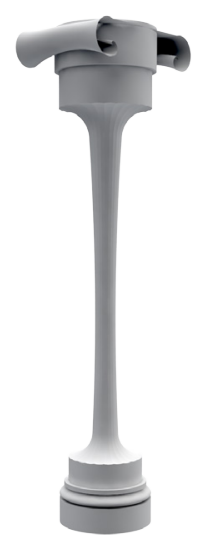

05

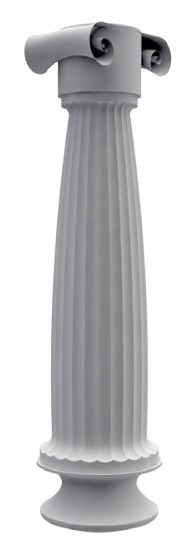

10

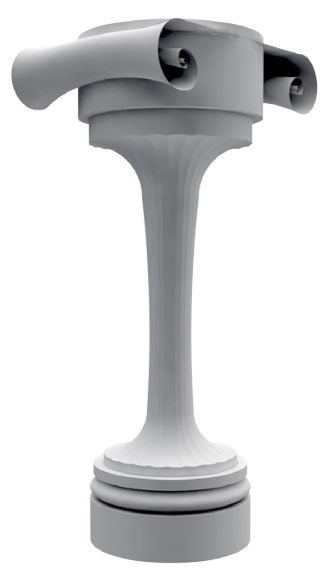

15

Figure 2.19: Composition of procedurally generated lonic columns

CHAPTER TWO 


\section{CORINTHIAN}

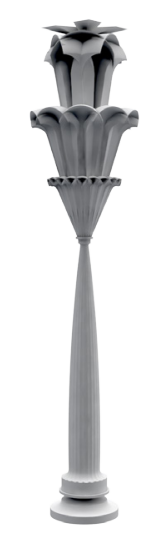

Output 01

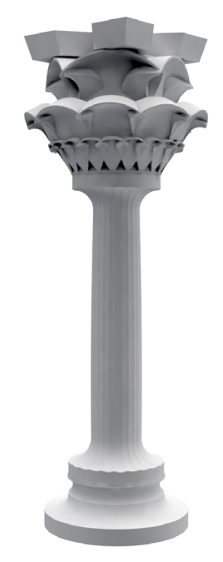

06

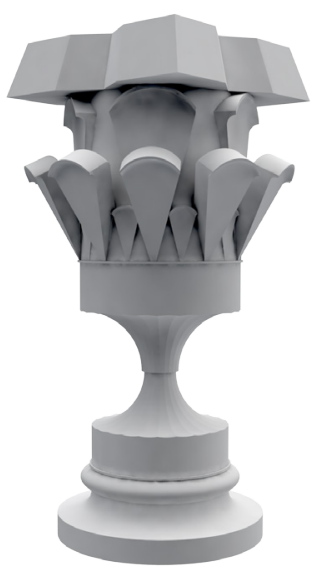

11

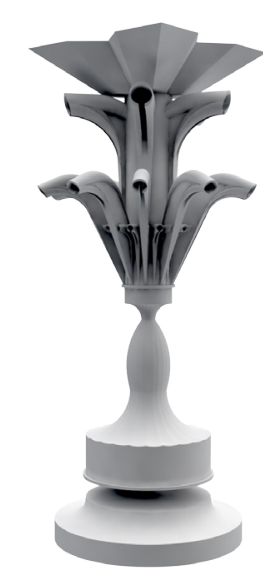

02

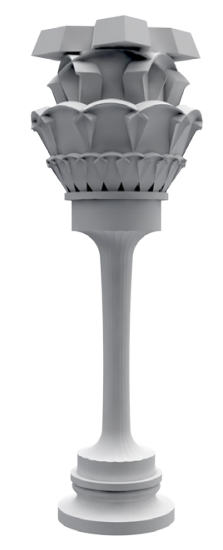

07

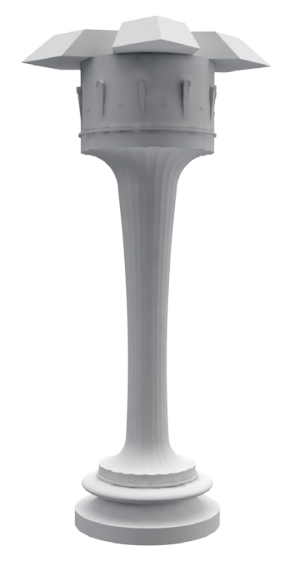

12

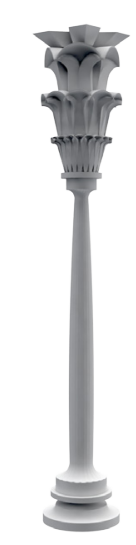

03

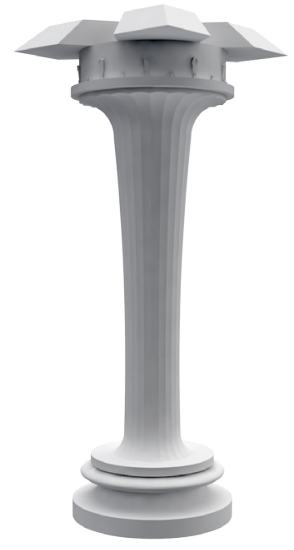

08

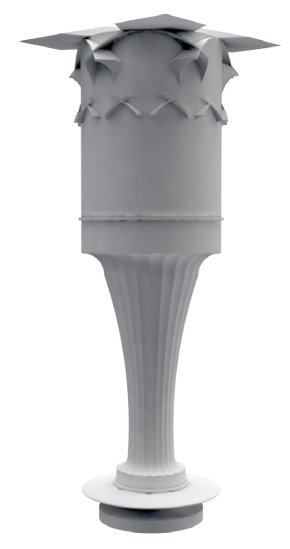

13

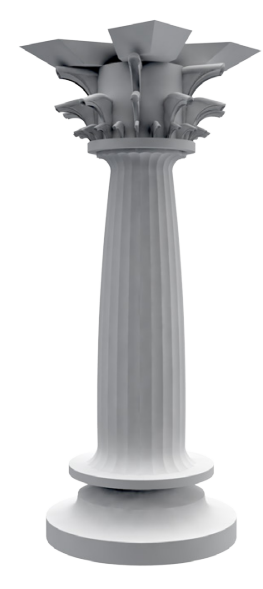

04

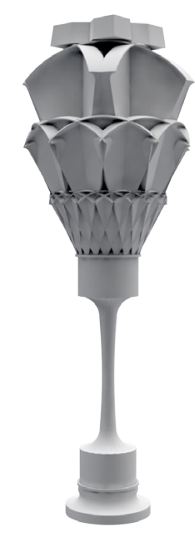

09

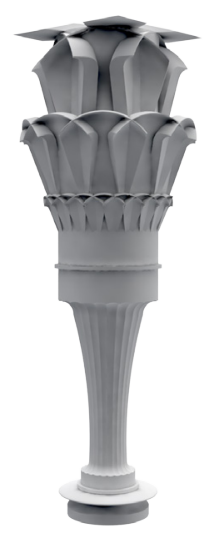

14

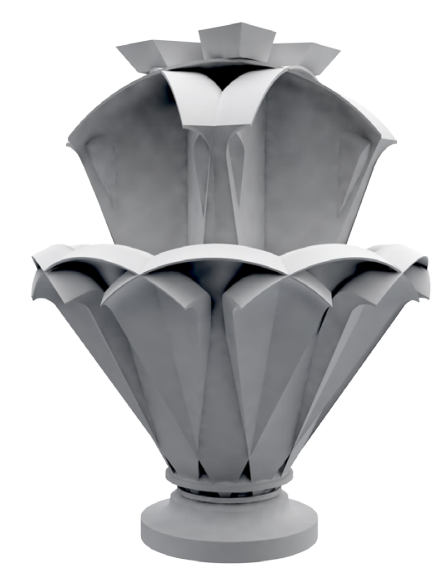

05

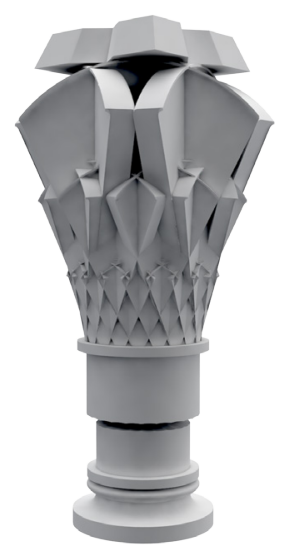

10

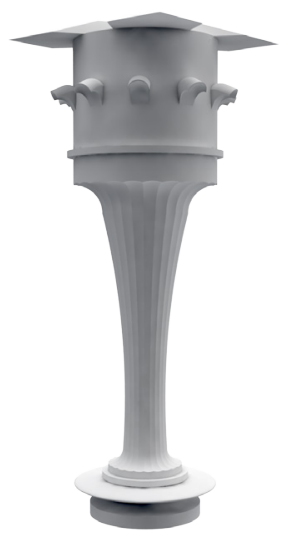

15

Figure 2.20: Composition of procedurally generated Corinthian columns 


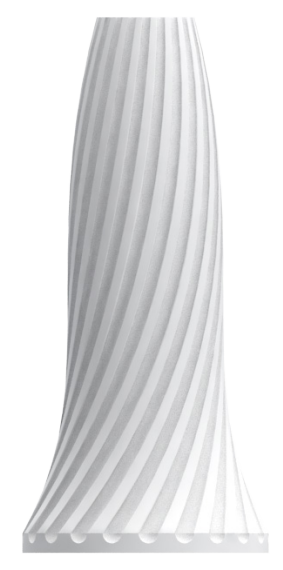

Seed 01

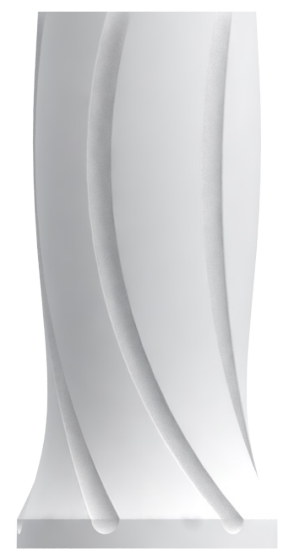

05

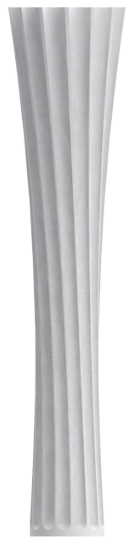

09

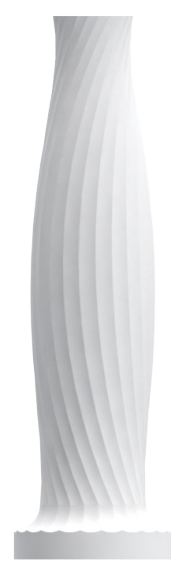

02

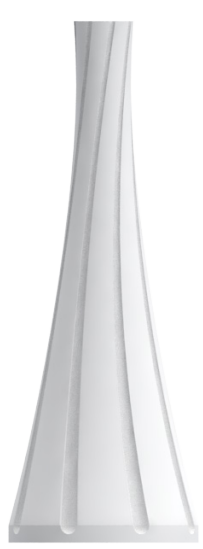

06

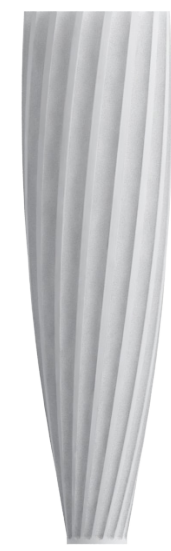

10

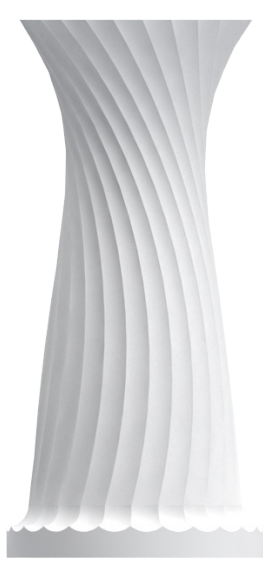

03

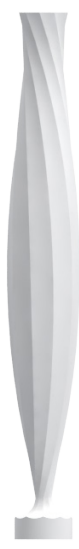

07

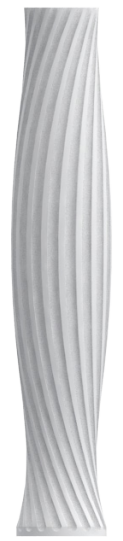

11

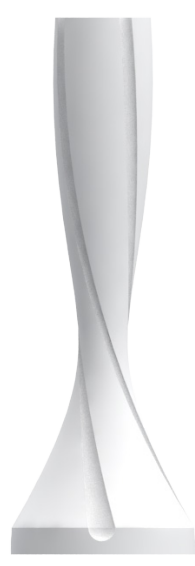

04

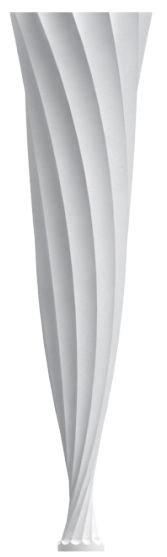

08

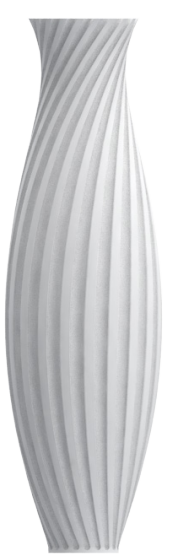

12

Figure 2.21: Composition of procedural column shaft outputs

CHAPTER TWO

A CITY IS NOT A TREE 


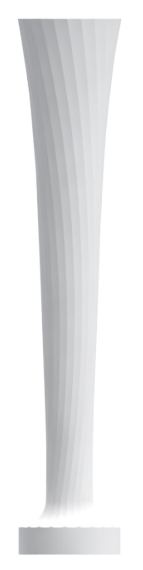

13

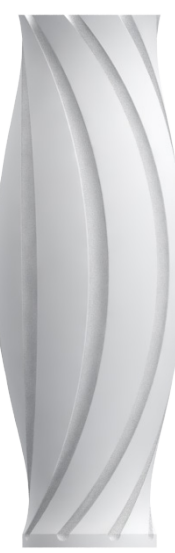

17

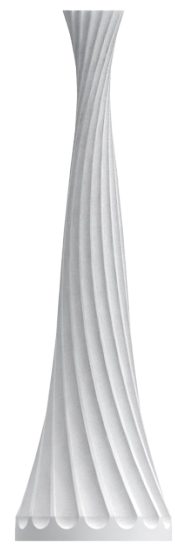

21

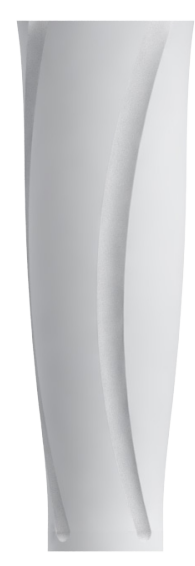

14

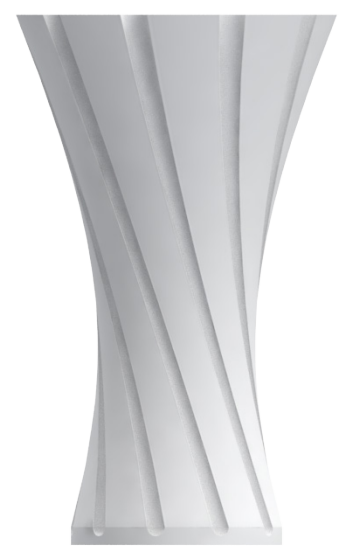

18

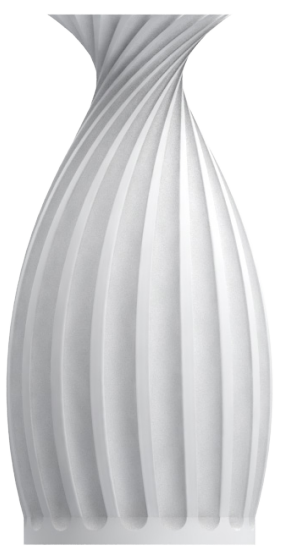

22

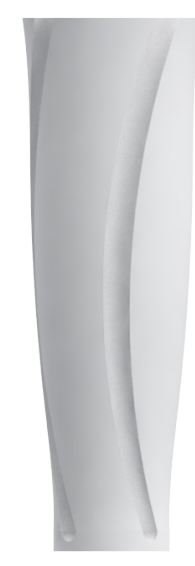

15

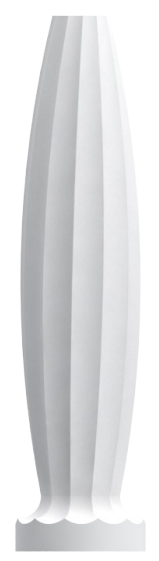

19

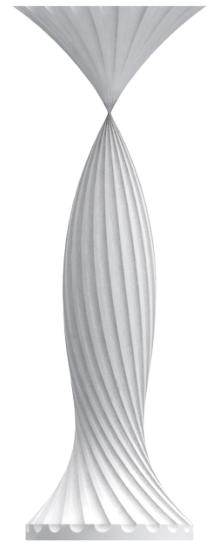

23

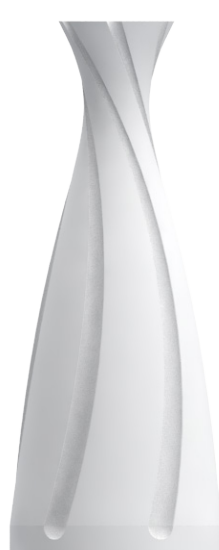

16

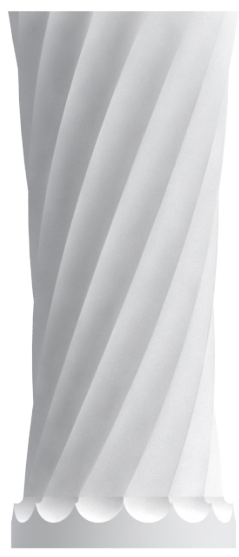

20

\begin{tabular}{|l|r|}
\hline Number of Spheres & $2-25$ \\
\hline Height Range & 6 - 14 Proportions \\
\hline Rotation & + - 90 degrees \\
\hline Scaling & 0.0 to 1.0 for Shaft Construction \\
\hline & 0.75 to 4.0 for Shaft Width \\
\hline
\end{tabular}

Figure 2.21: Composition of procedural column shaft outputs (cont.)

$$
\text { page } 034
$$




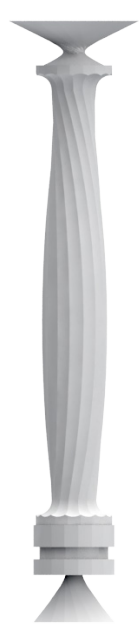

Seed 00

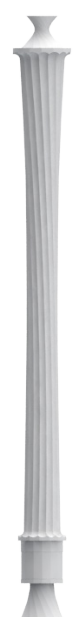

04

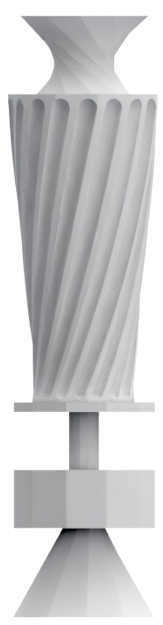

08

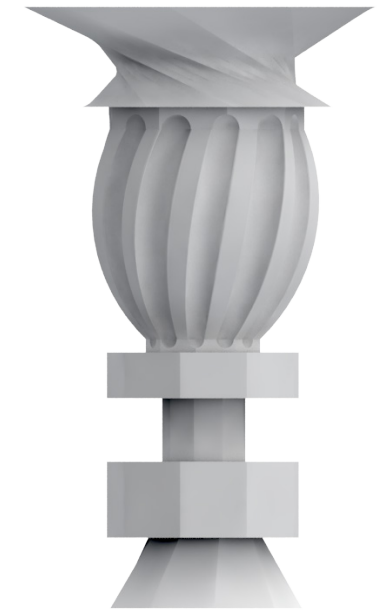

01

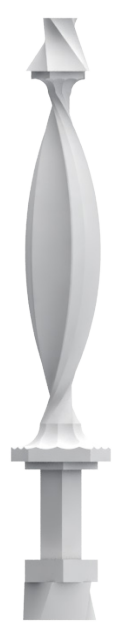

05

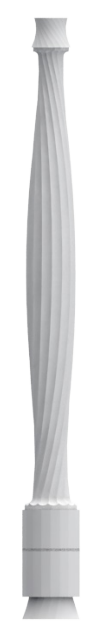

09

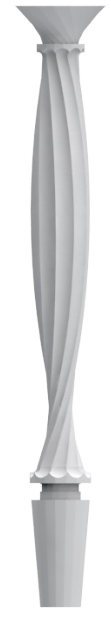

02

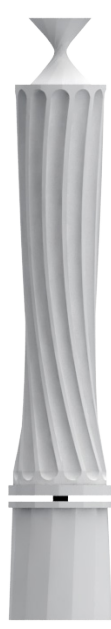

06

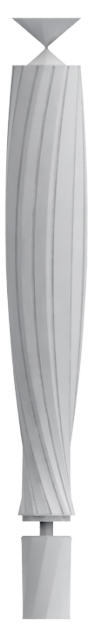

10

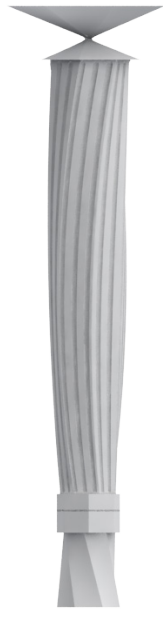

03

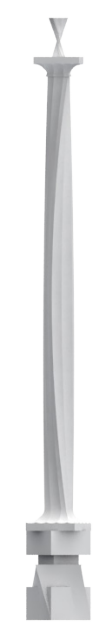

07

\begin{tabular}{|l|l|r|}
\hline Polygon Sides & & $3-20$ Sides \\
\hline Spheres & & $10-20$ \\
\hline Sphere Size & & $0.01-0.50$ * Base Circle \\
\hline Height Range & & $4-20$ Proportions \\
\hline Rotation & Shaft & + - 60 degrees \\
\hline & Base / Cap & $+/$ - 180 degrees \\
\hline Scaling & Cap & $0.5-1.5$ \\
\hline & Base & $0.1-1.0$ \\
\hline & Original Shaft & $0.5-1.5$ \\
\hline & Shaft Scaling & $0.5-1.0$ \\
\hline
\end{tabular}

Figure 2.22: Composition of procedural columns outputs - Standard Version

CHAPTER TWO

A CITY IS NOT A TREE 


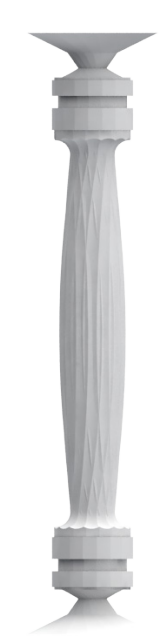

Seed 00

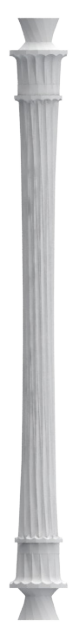

04

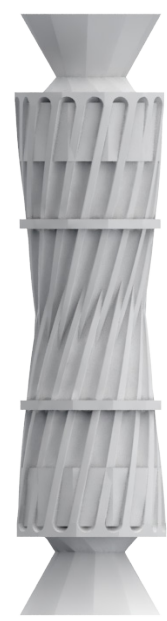

08

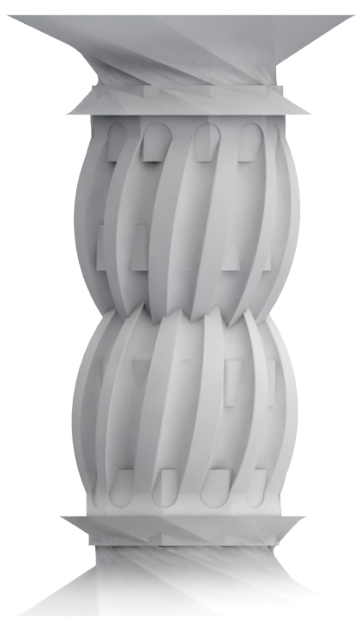

01

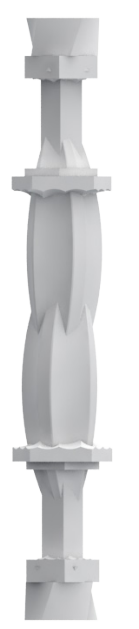

05

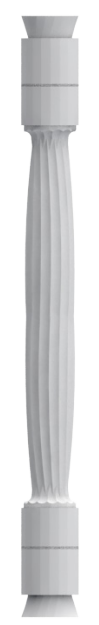

09

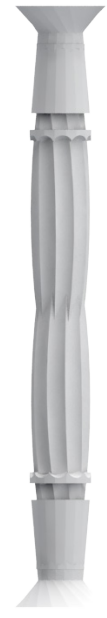

02

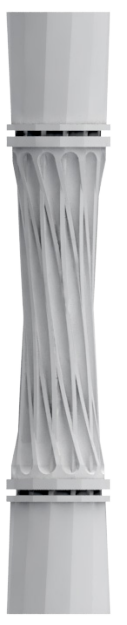

06

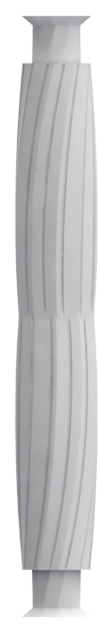

\begin{tabular}{|l|l|r|}
\hline Polygon Sides & & $3-20$ Sides \\
\hline Spheres & & $10-20$ \\
\hline Sphere Size & & $0.01-0.50$ * Base Circle \\
\hline Height Range & & $4-20$ Proportions \\
\hline Rotation & Shaft & $+/$ - 60 degrees \\
\hline & Base / Cap & $+/$ - 180 degrees \\
\hline Scaling & Cap & $0.5-1.5$ \\
\hline & Base & $0.1-1.0$ \\
\hline & Original Shaft & $0.5-1.5$ \\
\hline & Shaft Scaling & $0.5-1.0$ \\
\hline
\end{tabular}

Figure 2.23: Composition of procedural columns outputs - Mirrored Standard Version 


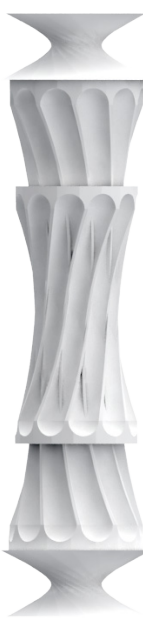

Seed 11

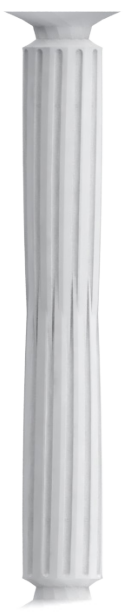

15

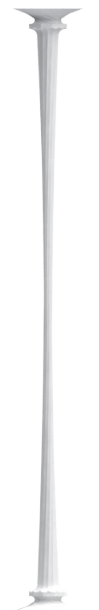

19

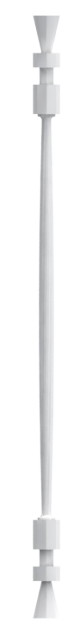

12

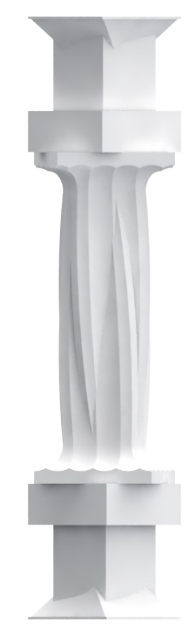

16

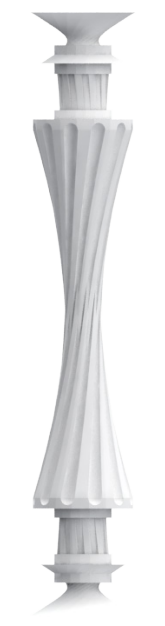

20

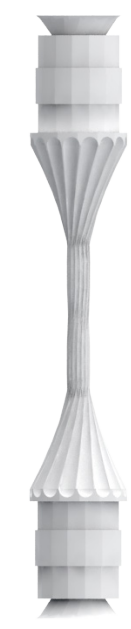

13

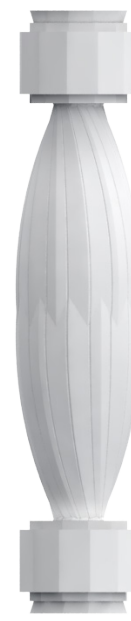

17

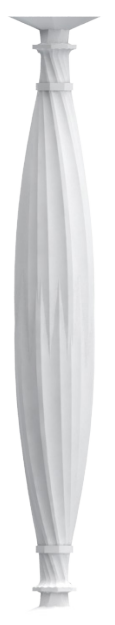

21

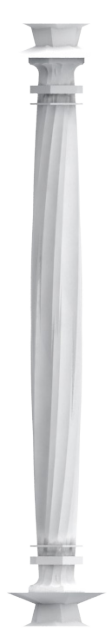

14

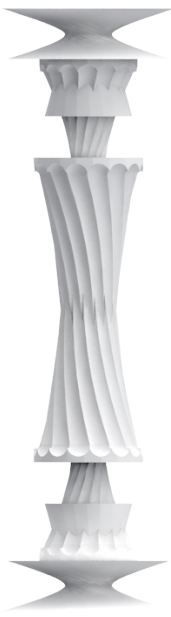

18

\begin{tabular}{|l|l|r|}
\hline Polygon Sides & & 3 - 20 Sides \\
\hline Spheres & & $10-20$ \\
\hline Sphere Size & & $0.01-0.50$ * Base Circle \\
\hline Height Range & & $8-20$ Proportions \\
\hline Rotation & Shaft & + - 60 degrees \\
\hline & Base / Cap & $+/$ - 180 degrees \\
\hline Scaling & Cap & $0.25-1.75$ \\
\hline & Base & $0.25-1.25$ \\
\hline & Original Shaft & $0.25-1.75$ \\
\hline & Shaft Scaling & $0.25-1.25$ \\
\hline
\end{tabular}

Figure 2.24: Composition of procedural columns outputs - Mirrored standard version, 0.25 Changes to scaling

CHAPTER TWO

A CITY IS NOT A TREE 


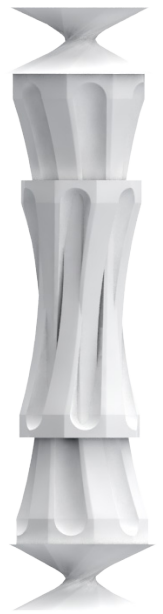

Seed 11

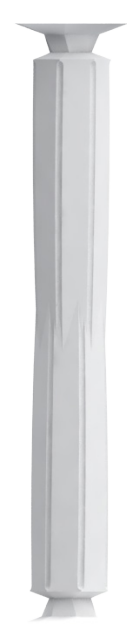

15

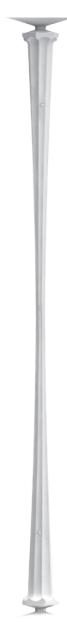

19

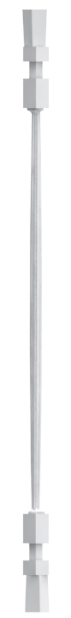

12

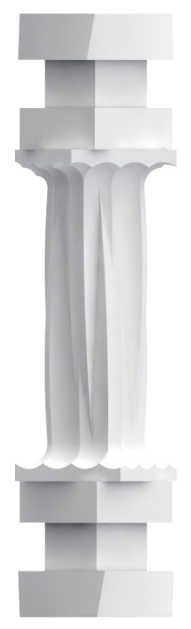

16

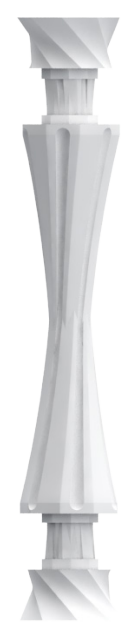

20

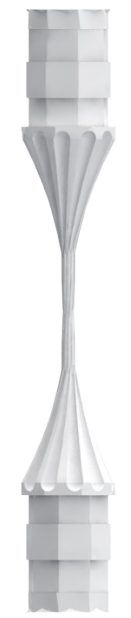

13

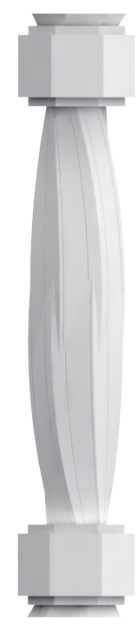

17

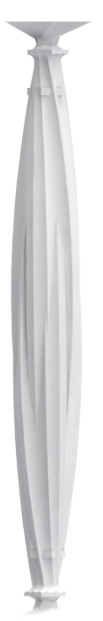

\begin{tabular}{|l|l|r|}
\hline Polygon Sides & & $3-15$ Sides \\
\hline Spheres & & $5-15$ \\
\hline Sphere Size & & $0.01-0.50$ * Base Circle \\
\hline Height Range & & $8-20$ Proportions \\
\hline Rotation & Shaft & $+/$ - 30 degrees \\
\hline & Base / Cap & $+/-90$ degrees \\
\hline Scaling & Cap & $0.25-1.75$ \\
\hline & Base & $0.25-1.25$ \\
\hline & Original Shaft & $0.25-1.75$ \\
\hline & Shaft Scaling & $0.25-1.25$ \\
\hline
\end{tabular}

21

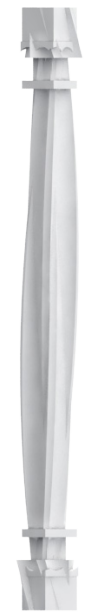

14

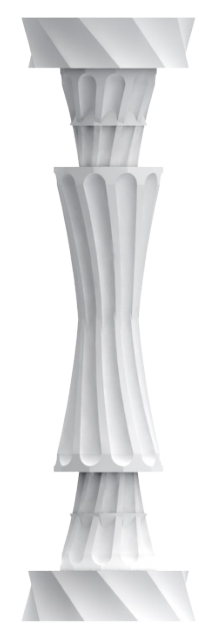

18 


\section{CHAPTER THREE




\section{INSPIRATION FOR CITY BUILDING}

Following a presentation from Chris White, the focus of this research pivoted. Chris White discussed his team's involvement in the recreation of 1930's New York City for Peter Jackson's King Kong, as well as non-specific urban environments for the Maze Runner series. The team involved in the creation of these environments included computer scientists and designers, amongst others. Notably, none of the team possessed a formal Architectural education or background (2016). Nonetheless, the work generated by the team at Weta Digital is a technical marvel and is nothing short of aweinspiring.

Viewing Chris' presentation piqued an interest in establishing whether or not Architects, and architecture students, could bring anything to this design process.

This work was incredibly interesting because of the way in which it displayed the application of procedural modelling techniques. Instead of presenting their tools Chris White displayed and explained the logic behind the system, and clearly explained the need for the program's application. Without the use of their tool, this modelling process would have taken years. Because this system acted to fully detail every building that it generated it also presented the opportunity for the film to locate itself in any part of the city. Peter Jackson was able to experiment with different camera placements, orientations, and more, without requiring the team to produce any additional work (White, 2016).

Figure 3.01: (Top) On set of King Kong (Universal Studios, 2006)

Figure 3.02: (Middle) Final shot in King Kong film (" ")

Figure 3.03: (Bottom) Image showing composition of building data (" ")
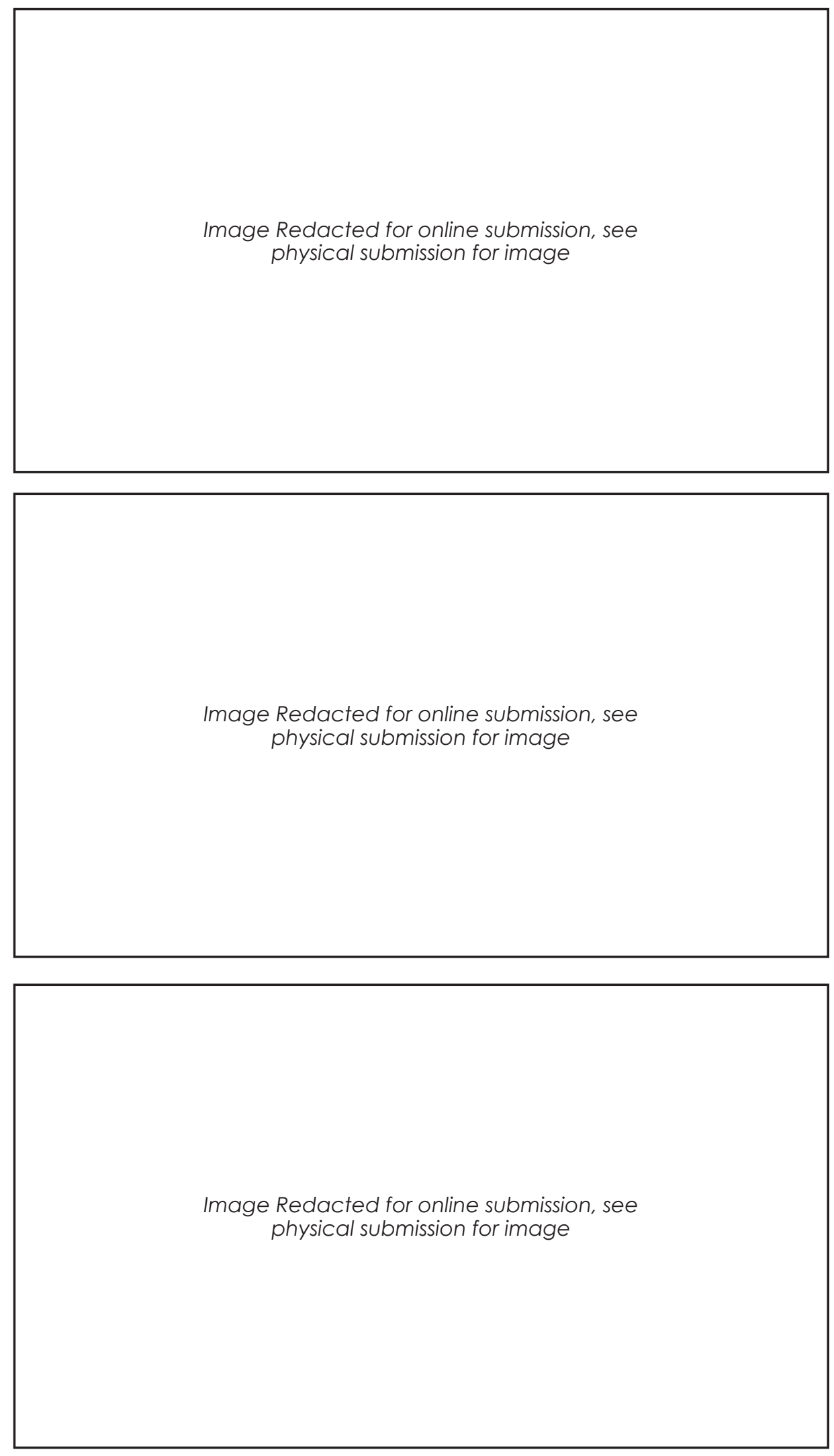


\section{CITY ANALYSIS}

Utilising Flux's site extractor tool an investigation into city blocks in Manhattan was undertaken (2016). Amongst others, some reasons for analysing Manhattan include:

- The quantity of reliable GIS data available

- The grid-based nature of Manhattan makes it easier to break down the data

- Manhattan as a borough, and NYC as a whole act as a global hub, holding top positions in areas including Banking, the Arts, and Media, to name but a few (Colvin, 2013).

While neither the borough nor the city as a whole, is a perfect representation of the world's built environments, both contain qualities that are prevalent in many and present an immensely useful data set.

Often the perception is that city blocks are incredibly dense. The reality is that they are incredibly varied, housing alleyways, carparks, courtyards, planting, arcades, empty lots, and so forth. Each of the previously listed elements develops organically for any number of reasons, which may include:

- Occupant demands/requirements

- Site history or context

- Building Code(s)

- Urban Planning requirements

- Gentrification

Capturing these details is where the research becomes necessary. The data obtained through this process displays years of development, which demonstrates how real world information can be manipulated and used to inform the creation of something new or different.

\begin{tabular}{|c|c|c|c|}
\hline & Average & Maximum & Minimum \\
\hline Minimum Area $\left(m^{2}\right)$ & 58 & 111 & 21 \\
\hline Maximum Area $\left(m^{2}\right)$ & 1113 & 3085 & 334 \\
\hline Area Variance $\left(m^{2}\right)$ & 1055 & 3035 & 272 \\
\hline Average Area $\left(m^{2}\right)$ & 224 & 468 & 141 \\
\hline Number of Buildings & 38 & 59 & 15 \\
\hline Minimum Perimeter (Lines) & 4 & 4 & 4 \\
\hline Maximum Perimeter (Lines) & 21 & 28 & 14 \\
\hline Perimeter Variance (Lines) & 17 & 24 & 10 \\
\hline Average Perimeter (Lines) & 8 & 11 & 5 \\
\hline Minimum Perimeter $(m)$ & 31 & 46 & 19 \\
\hline Maximum Perimeter $(m)$ & 155 & 291 & 82 \\
\hline Perimeter Variance $(m)$ & 124 & 250 & 47 \\
\hline Average Perimeter $(m)$ & 66 & 100 & 52 \\
\hline Minimum Height $(\mathrm{m})$ & 5 & 10 & 2 \\
\hline Maximum Height $(\mathrm{m})$ & 29 & 66 & 20 \\
\hline Average Height (m) & 16 & 19 & 13 \\
\hline Variance in Heights $(m)$ & 23 & 61 & 13 \\
\hline
\end{tabular}

Table 1: Values from 27 NYC Blocks 



\section{GRID BASED CITY}

Following the Flux investigation, the first design iteration focused on arrayed rectangles. Each rectangle was divided, with the first version generating a $5 \times 5$ grid.

A goal of the system was to join the subdivided surfaces through randomisation to establish different building footprints. This process worked up to a $4 \times 4$ surface, but the smaller grids were incapable of recreating the qualities of city blocks. The complexity involved in any system larger than $4 \times 4$ became unreasonable and meant that this was not a practical approach.

Another exercise undertaken with this system involved the randomised removal of surfaces from the larger systems (Fig. 3.05). The outputs generated lacked any real control or depth. However, the investigation succeeded in highlighting alternative ways to approach the challenge. This work also demonstrated a need to focus on smaller scale definitions such as individual blocks, rather than an entire city.

\section{FOOTPRINT GENERATOR 01}

The next stage involved the construction of a single block footprint generator. This process made use of a base surface, and four randomly placed points to define the corner surfaces. Through various operations, the system would also establish a new block between each of the corner surfaces. For reasons relating to the mathematical operations taking place, this iteration of the system was unsuccessful. Outcomes like this present lessons and new ideas for further developments, making them a necessary part of the process.

At this point of increasing complexity hand drawn diagrams became crucial for planning and communicating ideas. The ideas themselves are not overly complex, but their composition within Grasshopper is tough to grasp for anyone unfamiliar with the software.

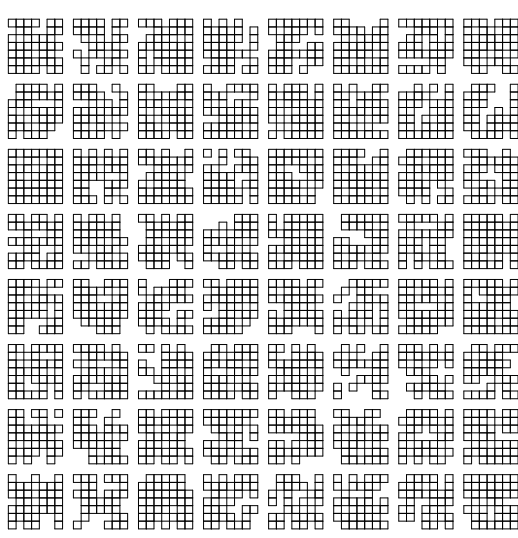

Seed 01 / 25\% Reduction

Figure 3.05: Outputs from basic city definition

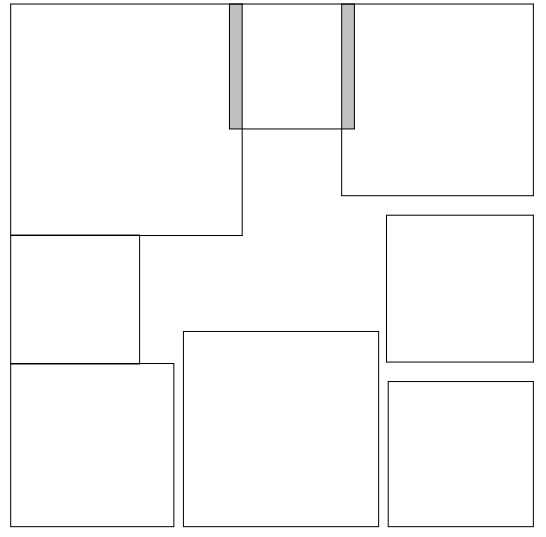

Seed 01

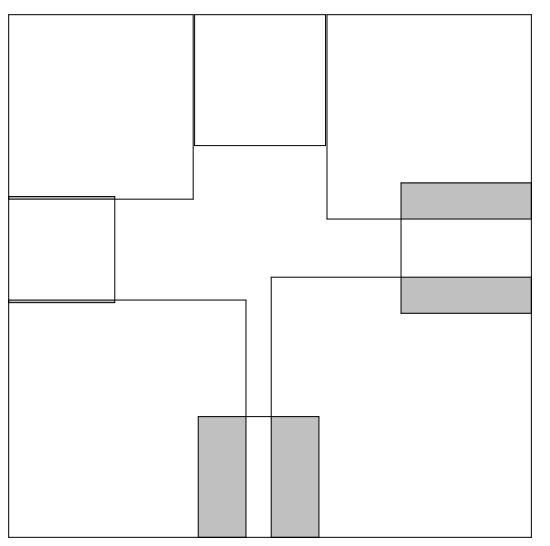

03

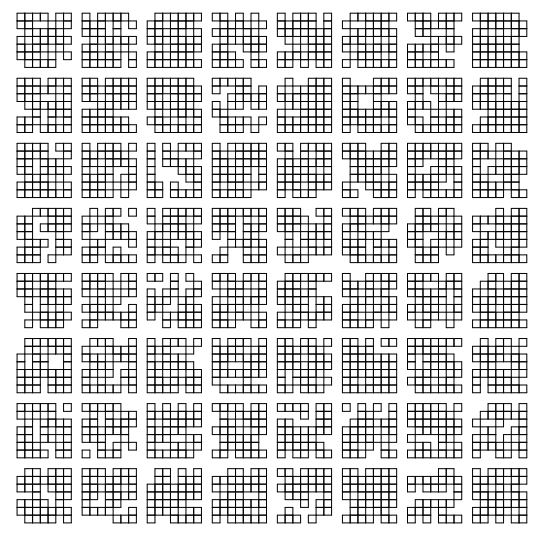

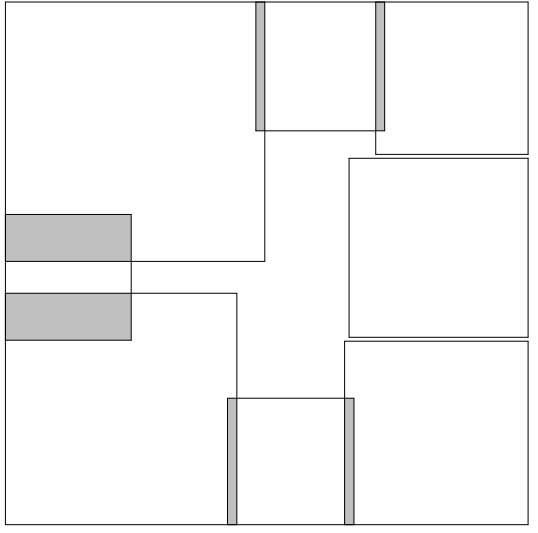

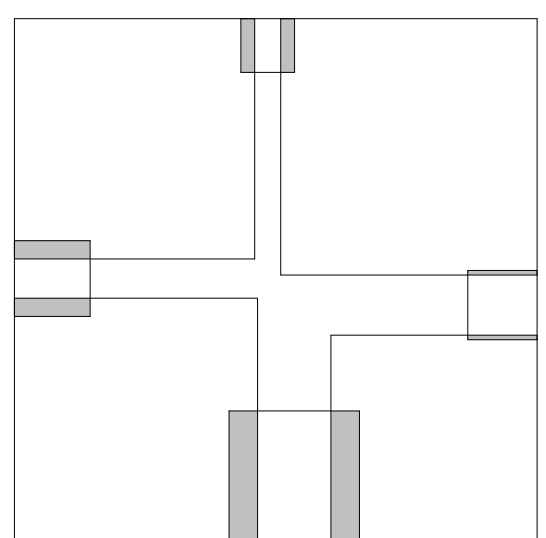

04

Figure 3.06: Outputs from basic city extrusion definition 


\section{HYPERBOLIC CITY}

While the individual blocks were under construction, systems were also developed to consider building heights. In the first instance, a single attractor point was placed randomly amongst the building footprints. Distance from the point to the centre of each footprint was then established, with the footprints extruded based on a modified version of this value.

Most cities have a focal point, but this system unintentionally took the idea to extremes. Because there were no restrictions on the attractor placement, it could situate itself within millimetres of the centre of any given building footprint. The resultant extrusion would become excessively large, dwarfing all others.

Resolving this issue could be attempted in a variety of ways, such as restricting the locations in which any attractor can place itself. Other approaches may include utilising multiple attractors and averaged distance values to inform any extrusion. Combining either of these methods with a value jitter (shuffle) could further reduce the occurrence of outlandish extrusions.

\section{BASIC EXTRUSION}

As a simplification of the attractor-based extrusion, an attempt was made to create city blocks with mixed height buildings. Again, this was a return to the smaller scale to focus on controlling the outcomes.

This system utilised a single extruded form. Next, randomised values generate within a user defined range, which informs the number of times the extrusion would be moved vertically from each starting position. While rudimentary this exercise produced more useful and developable outputs than the attractor-based system.

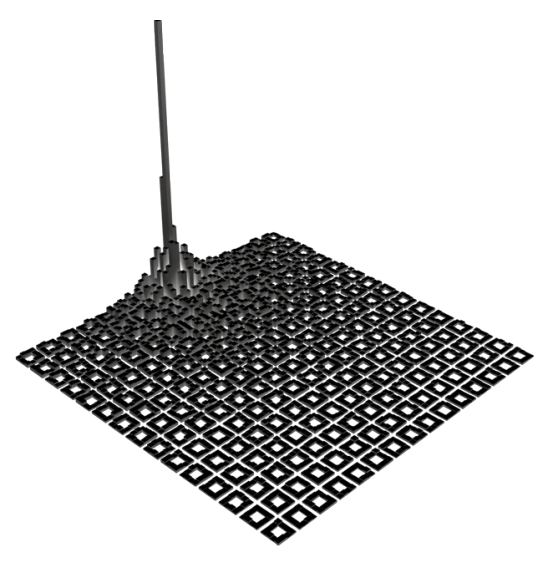

Seed 01

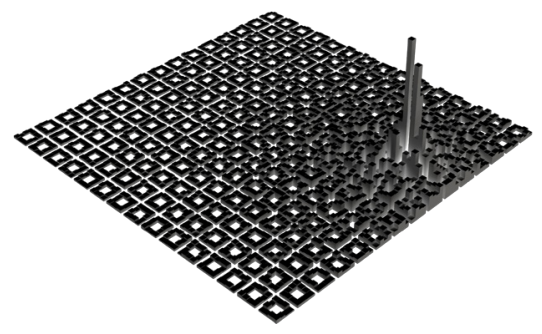

03

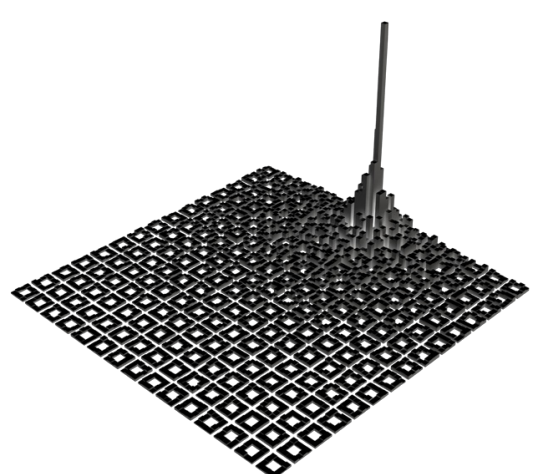

02

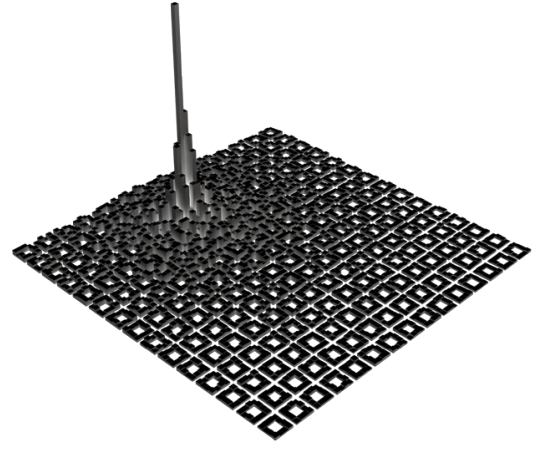

Figure 3.07: Composition of attractor based extrusion outputs

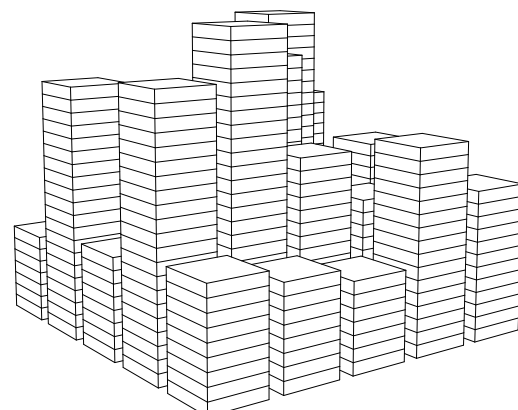

Seed 01

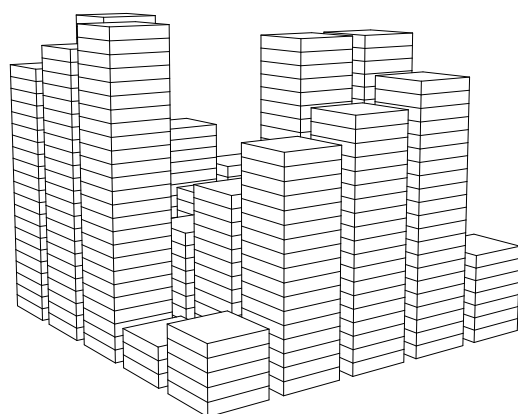

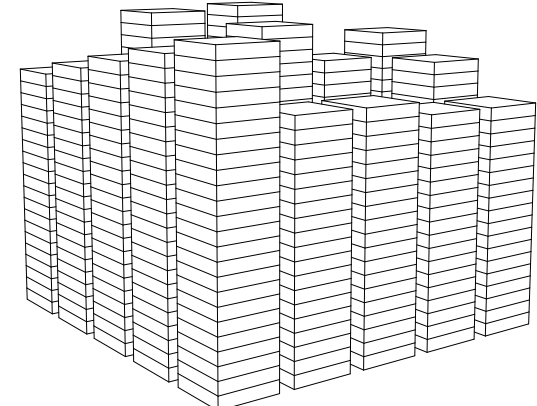

02

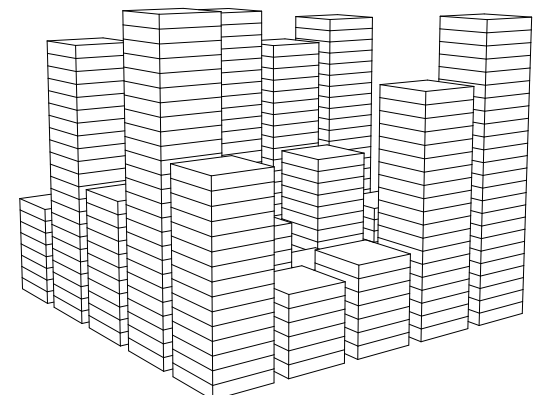

04
Figure 3.08: Composition of early footprint generator outputs 


\section{FOOTPRINT GENERATOR 02}

The second footprint generator developed on ideas explored in the previous iteration. The process for generating this block was as follows:

- A rectangular surface is created

- The surface is scaled to create a restricted space for the random generation of a point and four new surfaces are formed between each corner point and the placed point

- The new surfaces assigned an orientation, including positive and negative $X / Y$ direction values

- The surfaces are divided in the specified direction, based on a minimum and maximum building width. The exterior edge block within each of these surfaces is then selected

- The selected block is then divided in the alternate direction to the original block. For example, if the original block was in the $X$ direction the edge block will be divided in the $Y$ direction.

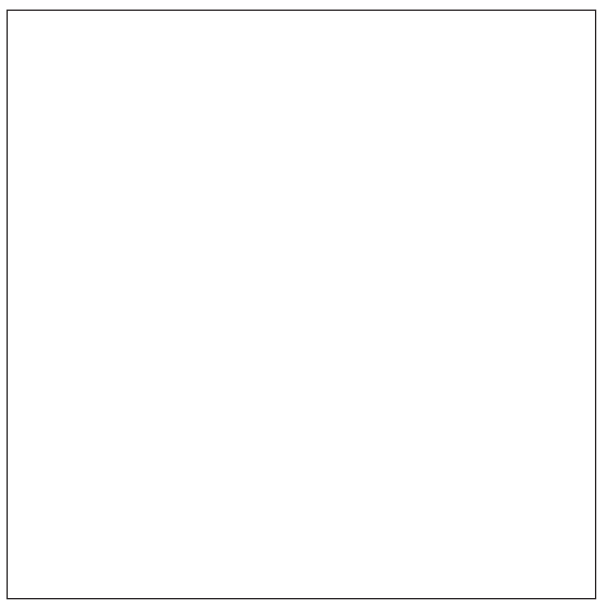

Step 01

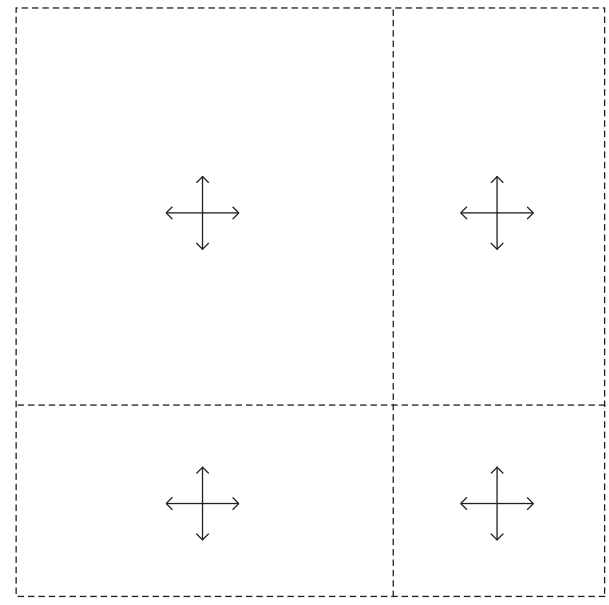

03

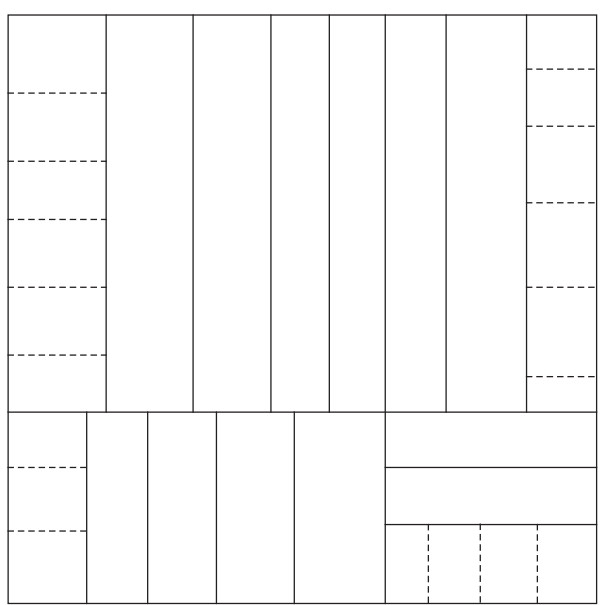

05
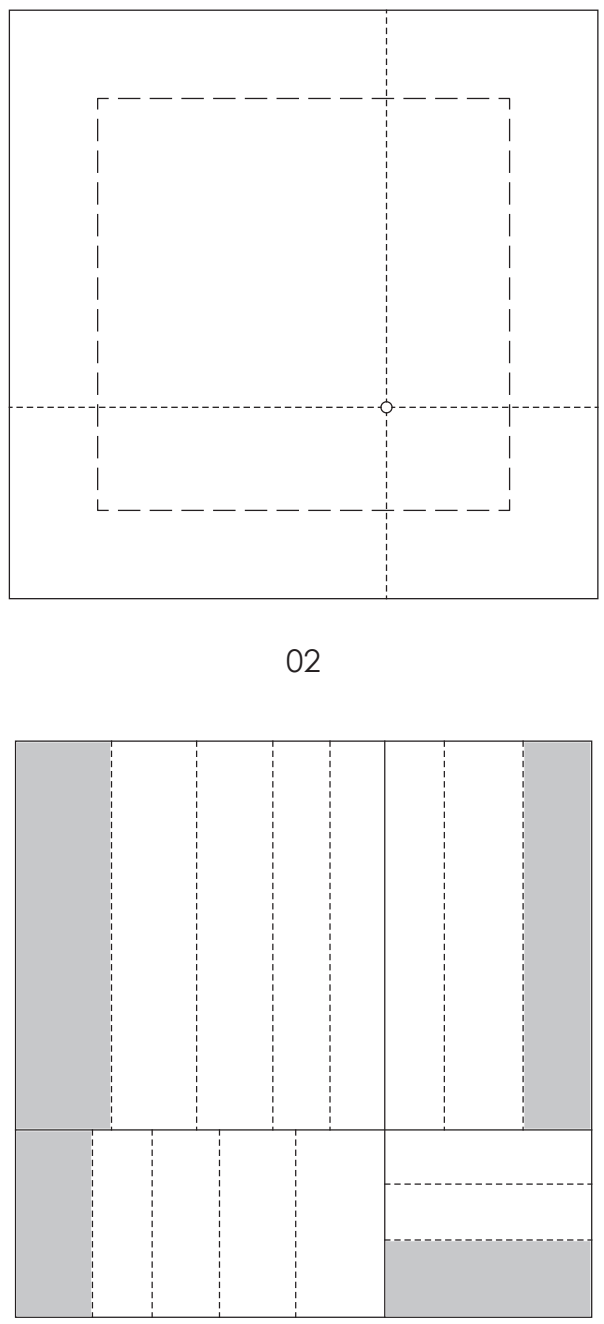

04

Figure 3.09: Construction logic for creating blocks 


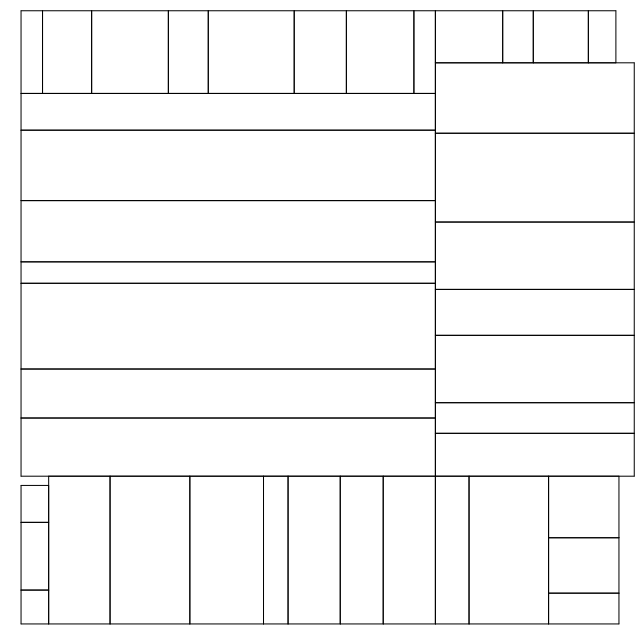

Seed 01

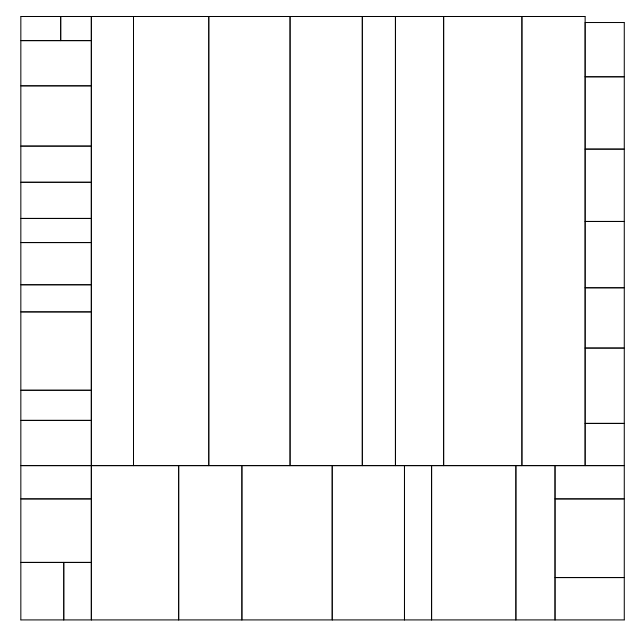

04

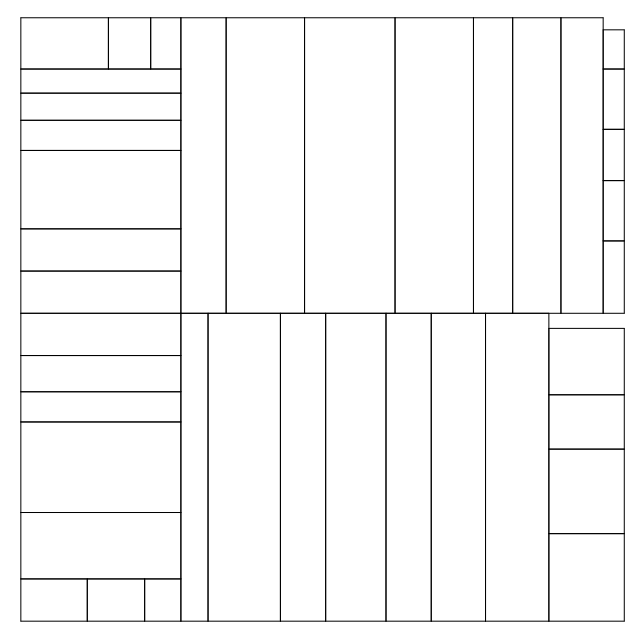

07

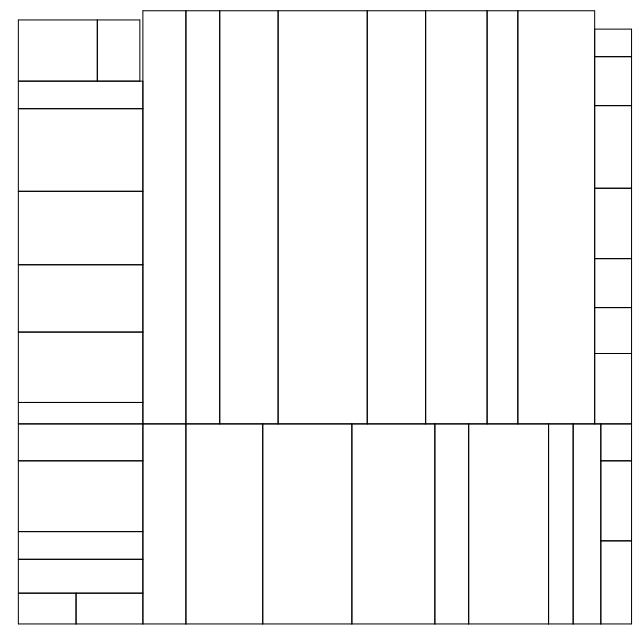

02

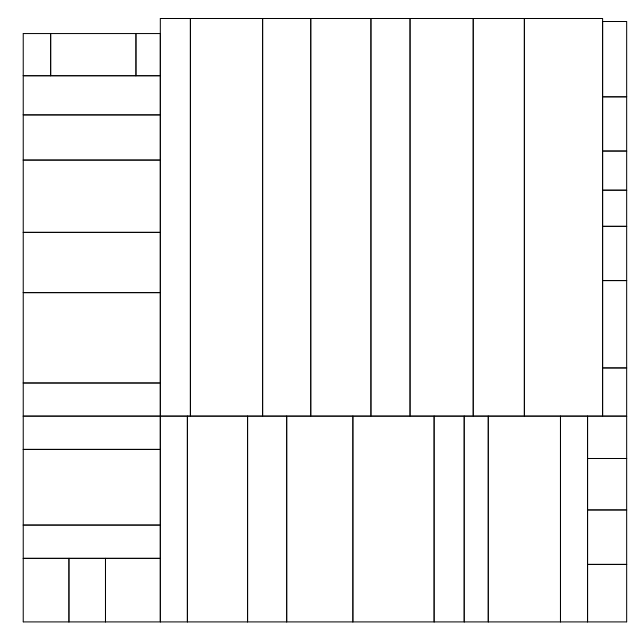

05

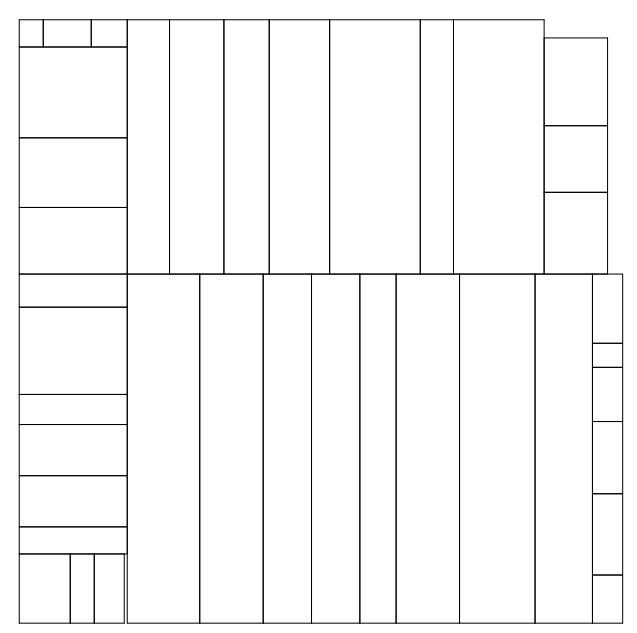

08

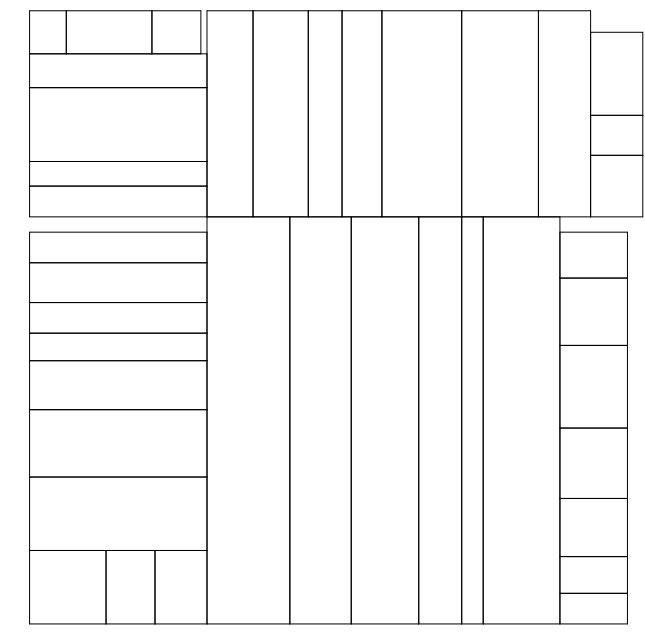

03

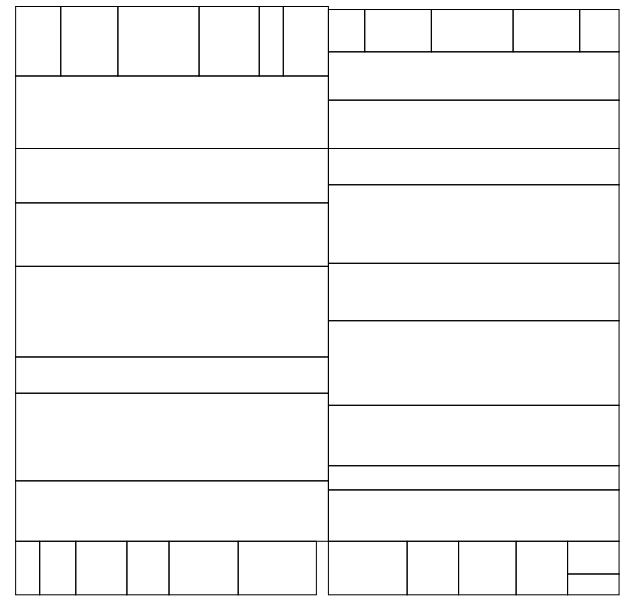

06

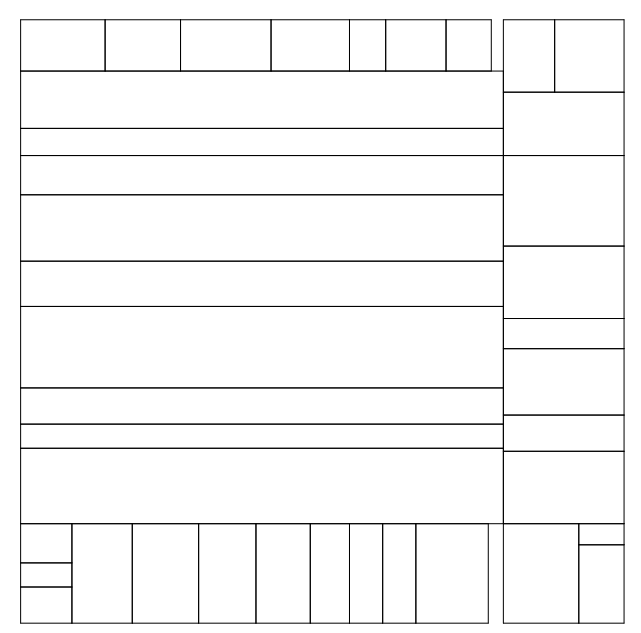

09

Figure 3.10: Composition of outputs from block creator 


\section{CHAPTER FOUR \\ SINGLE CITY BLOCK ARTICULATION}




\section{ITERATION ONE}

The first successful iteration of this work established several processes that have continued through the development of each new stage. As shown in Fig. 4.01 the block was established as follows:

- A rectangular surface is created

- Surface is divided into equal segments, and a point is randomly placed in each segment

- New surfaces are formed between each corner and the placed points

- The internal corner of the each surface is extended in a clockwise direction to establish a new line, and surface

- The new surfaces are divided into equal portions to create building footprints

- The building footprints are scaled from the centre to create a more staggered street edge

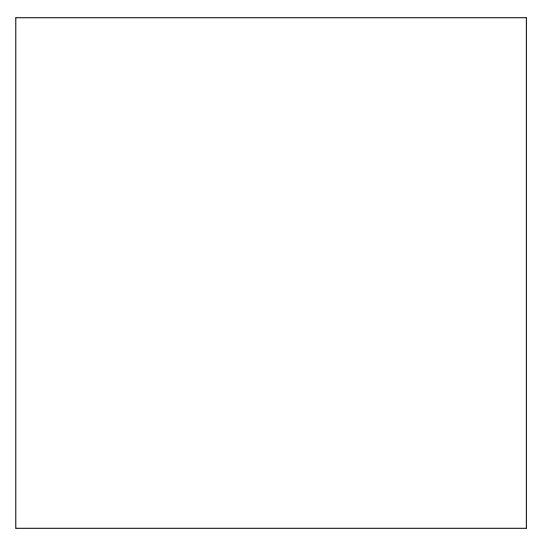

Step 01
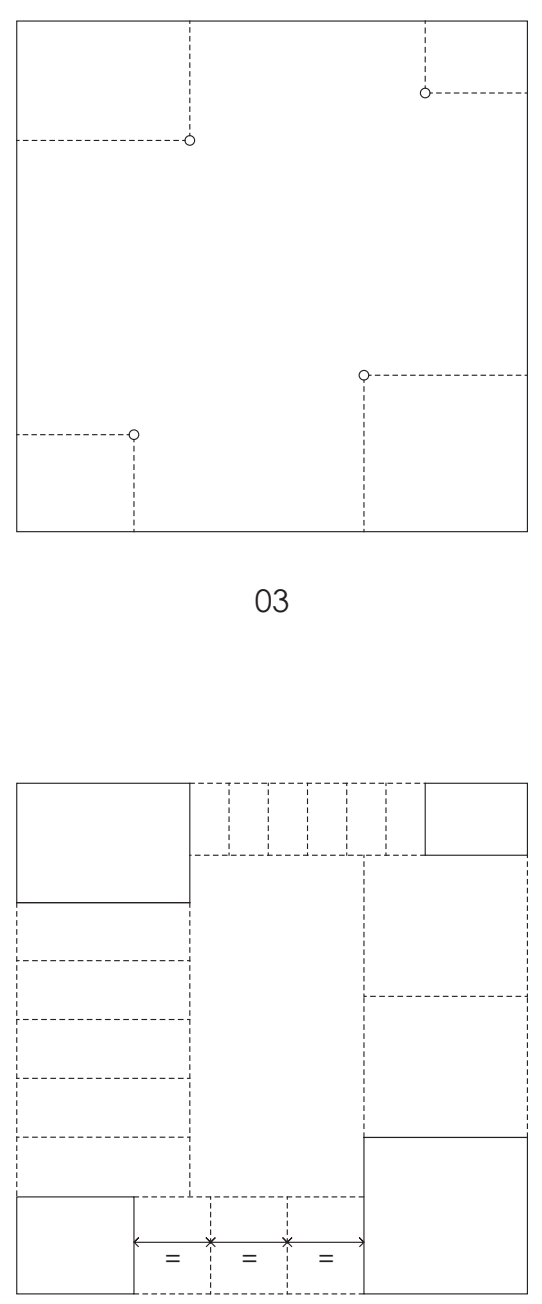

05
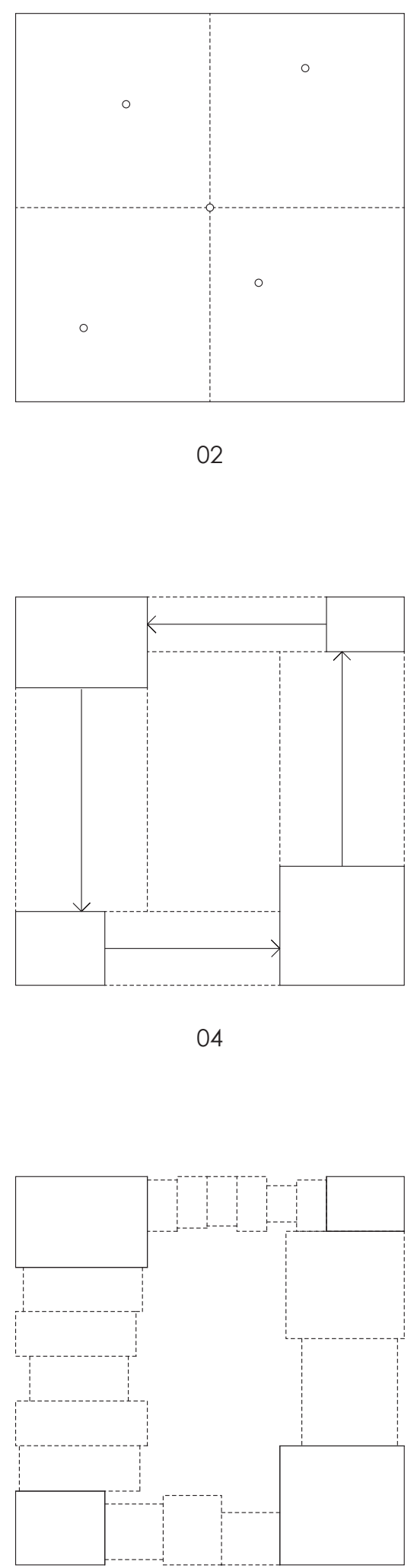

06 


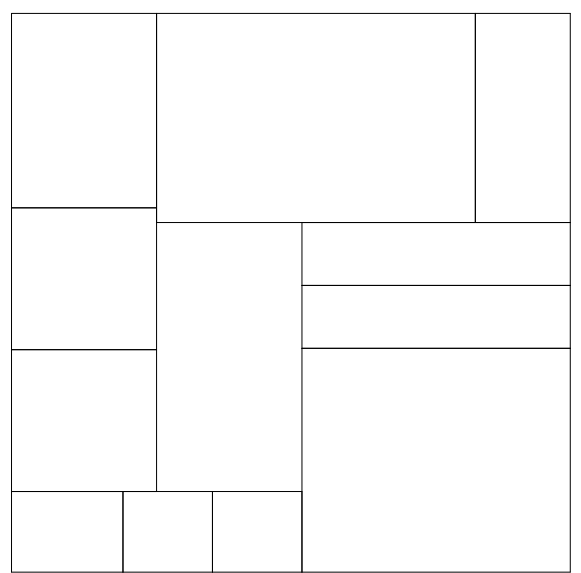

Seed 01

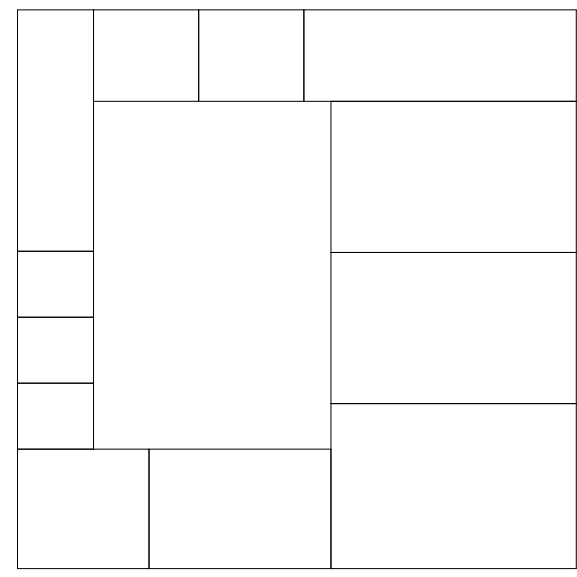

05

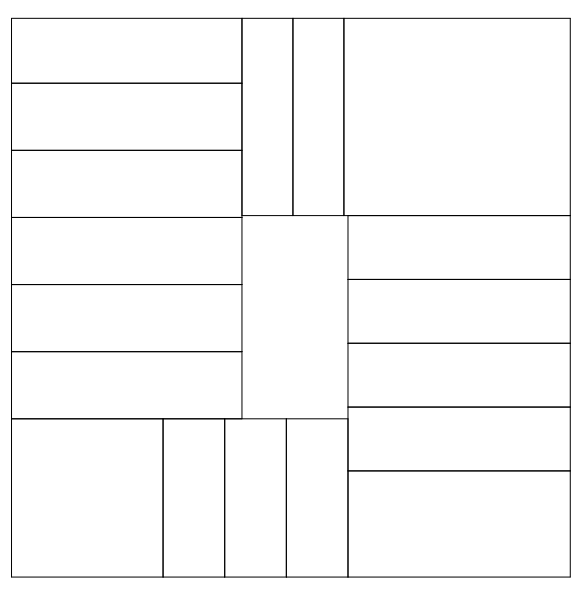

09

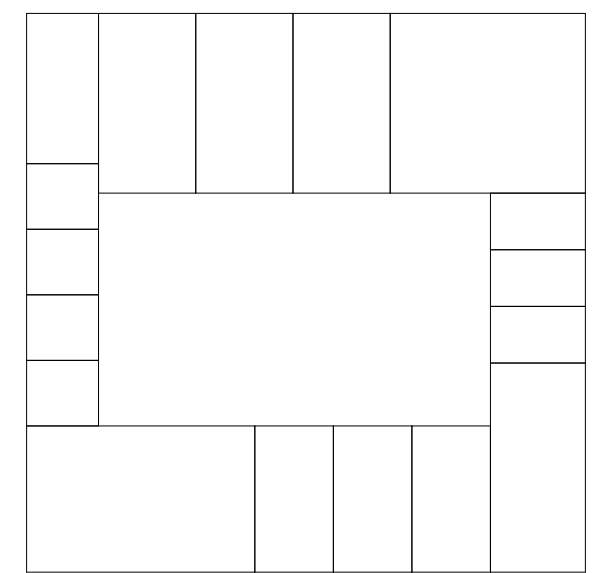

02

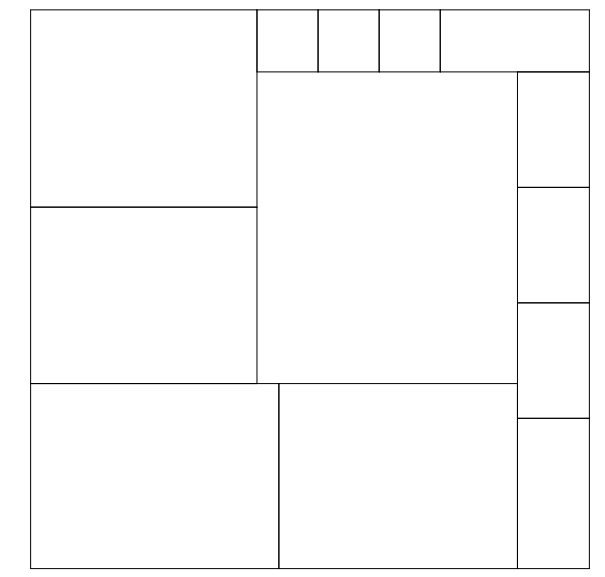

06

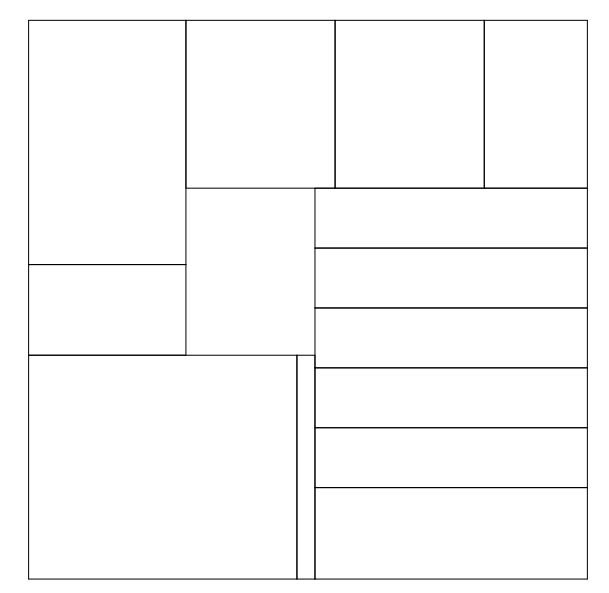

10

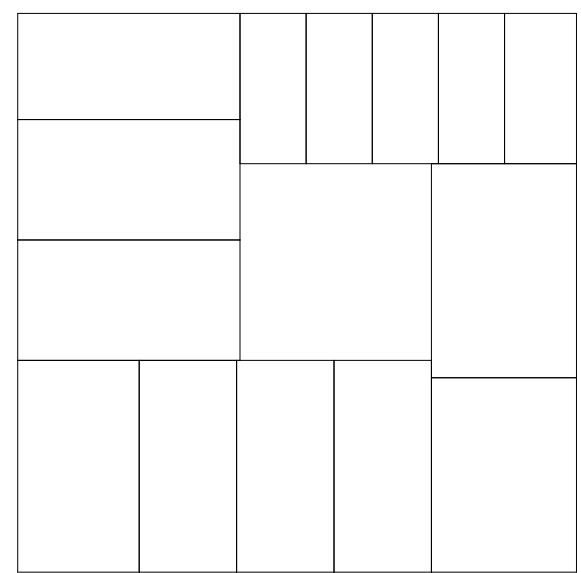

03

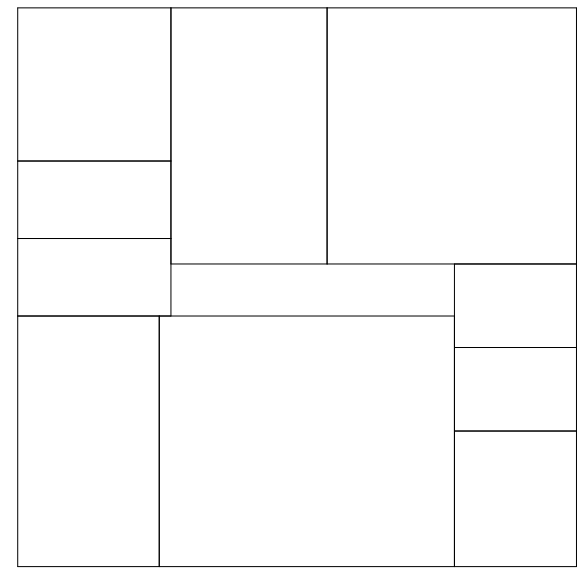

07

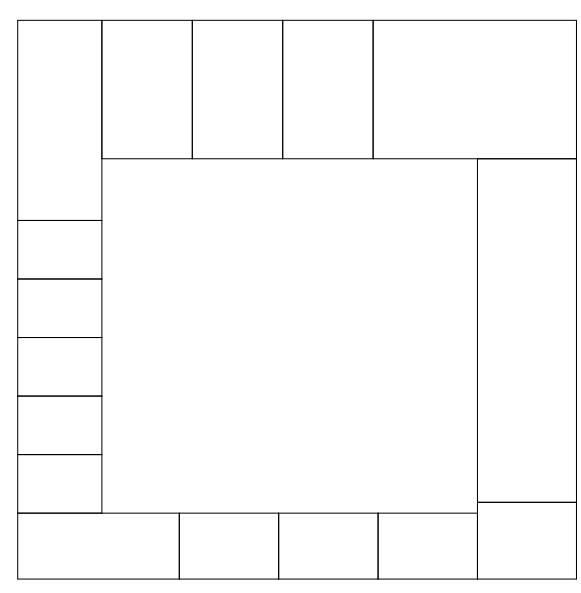

11

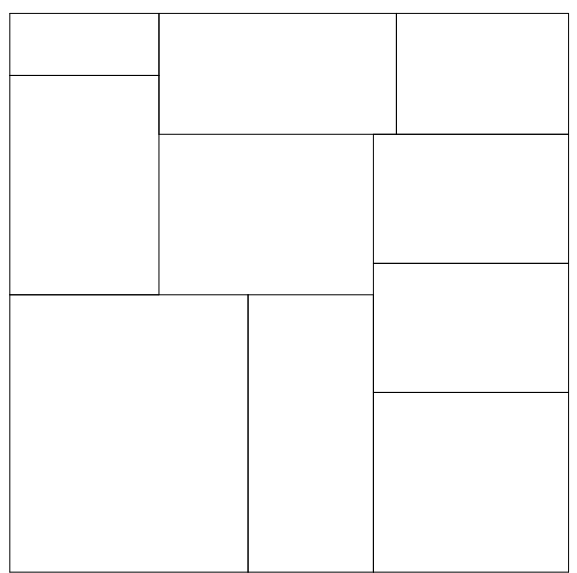

04

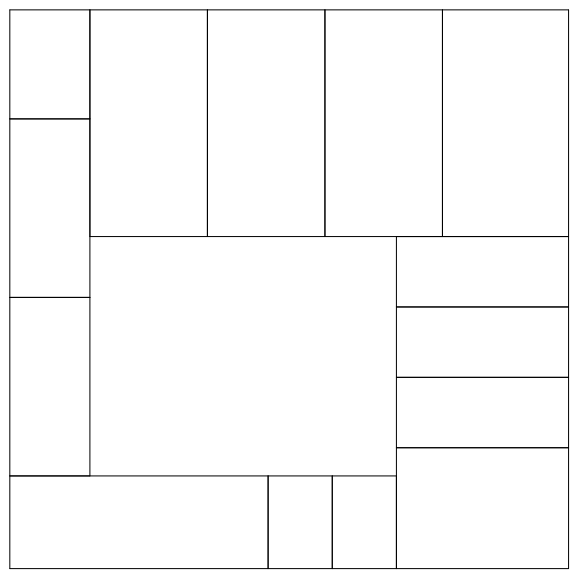

08

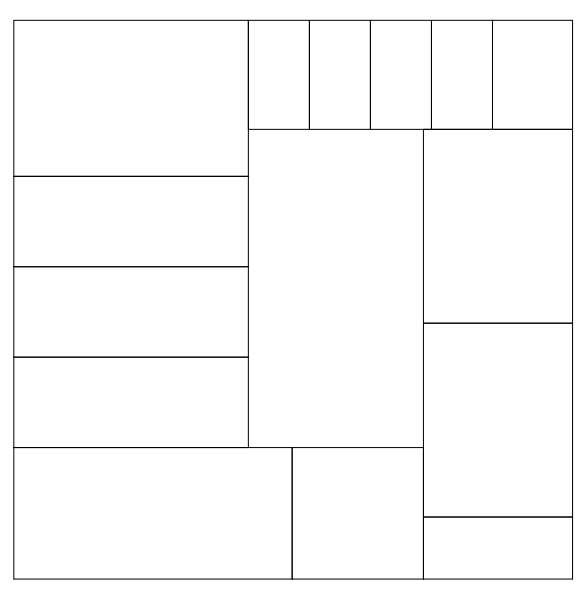

12

Figure 4.02: Compilation of Iteration One outputs - Internal scaling 0.1 - 0.5

CHAPTER FOUR

A CITY IS NOT A TREE 


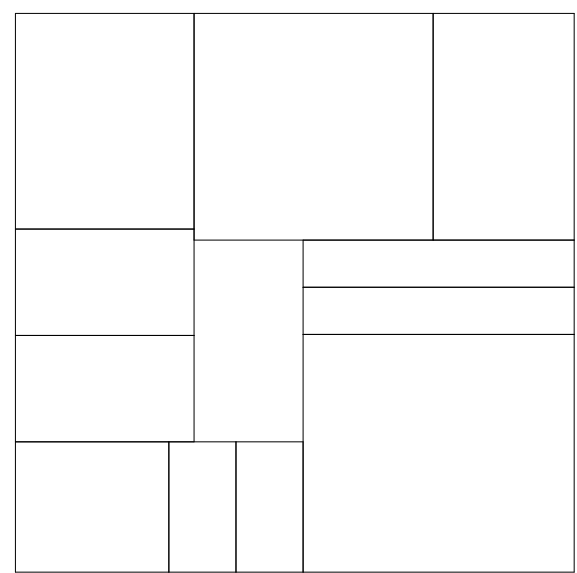

Seed $01 / 0.2-0.5$

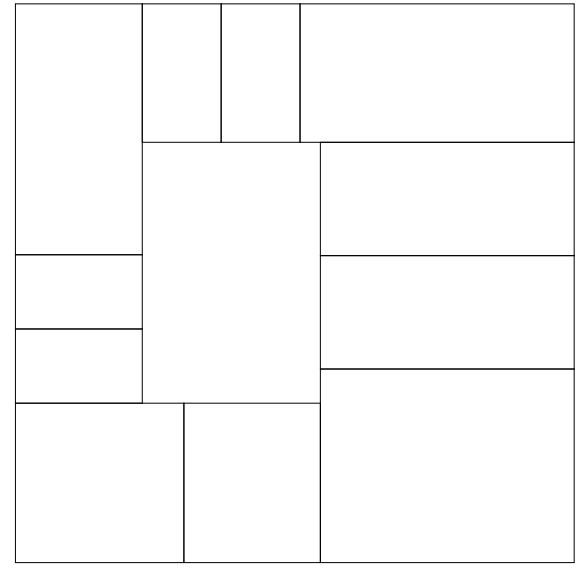

Seed 05 / 0.2 - 0.5

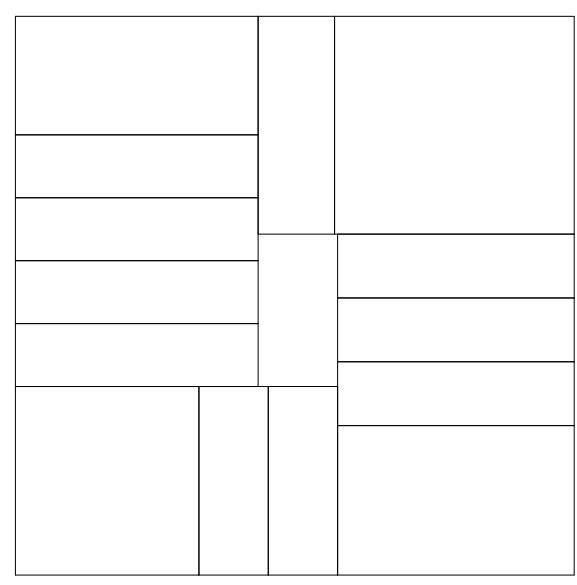

Seed $09 / 0.2-0.5$

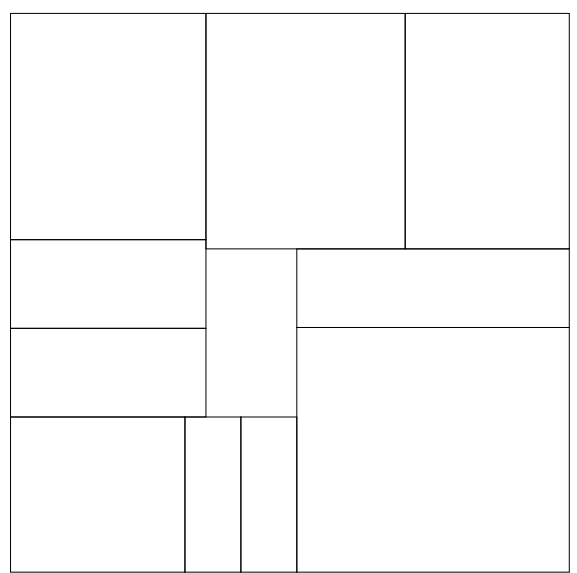

$0.25-0.5$

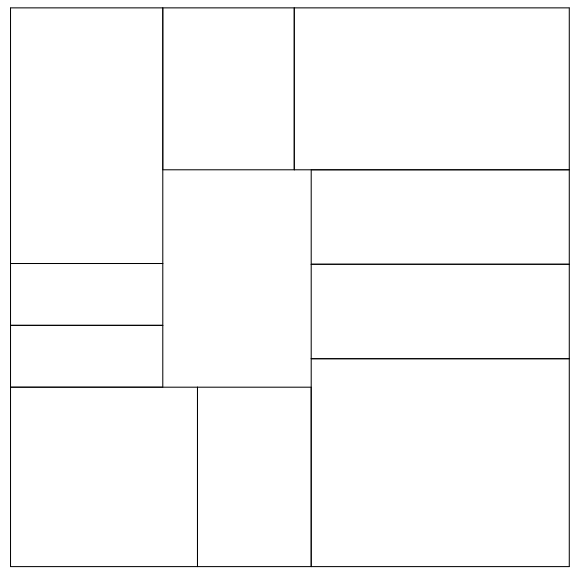

$0.25-0.5$

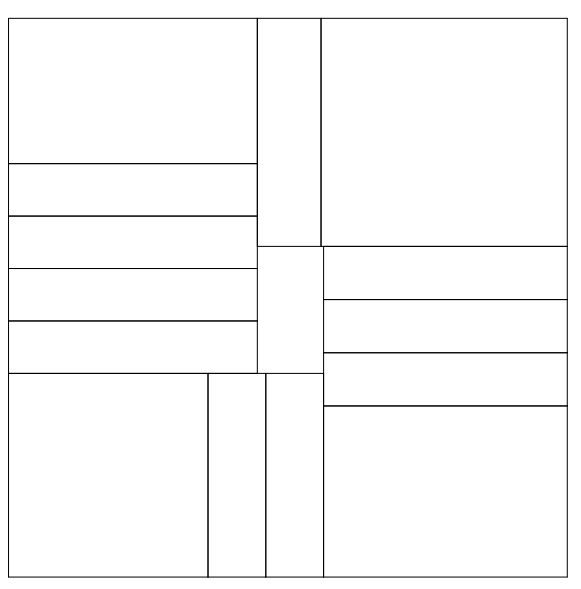

$0.25-0.5$

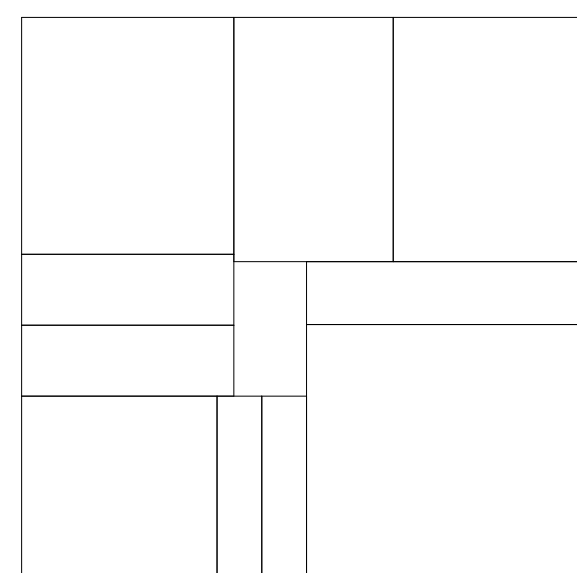

$0.30-0.5$

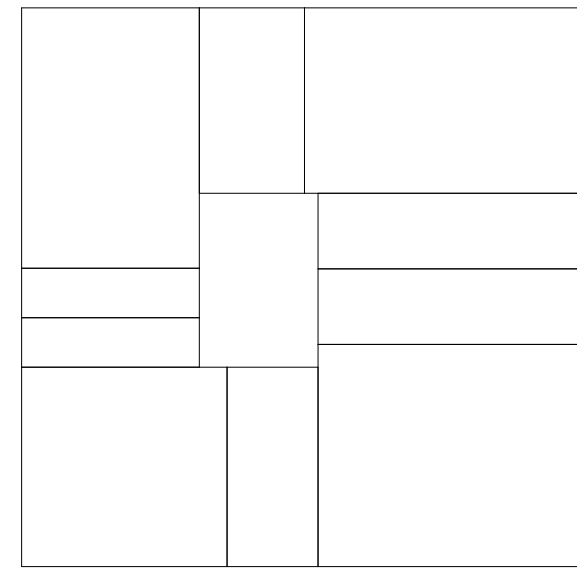

$0.30-0.5$

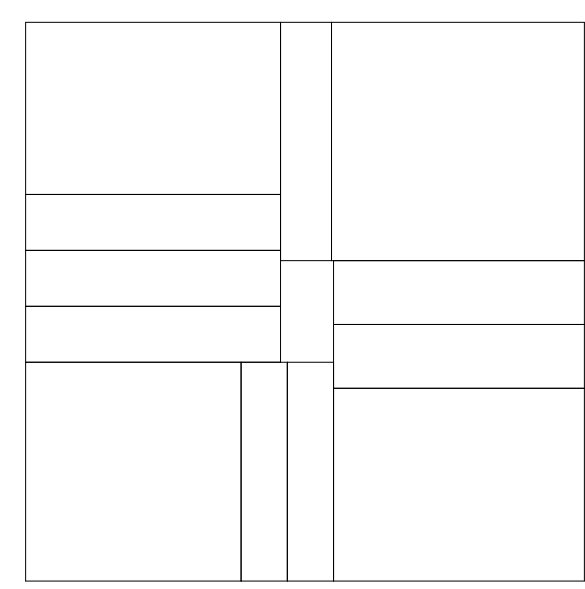

$0.30-0.5$

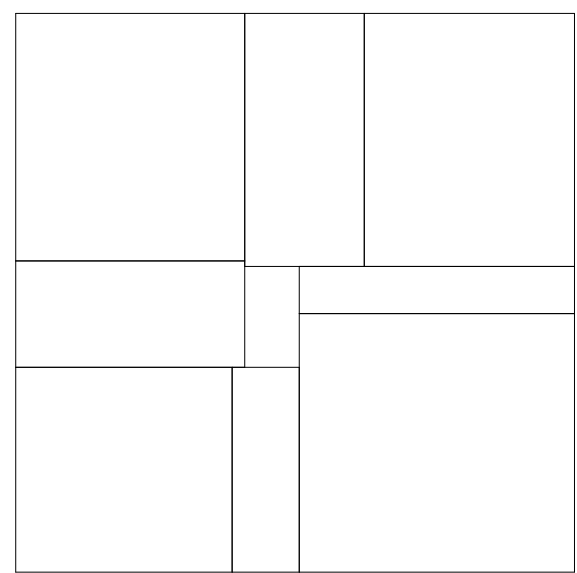

$0.35-0.5$

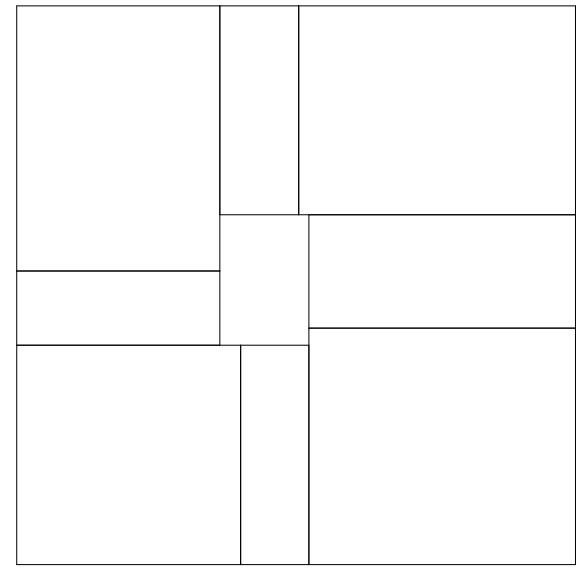

$0.35-0.5$

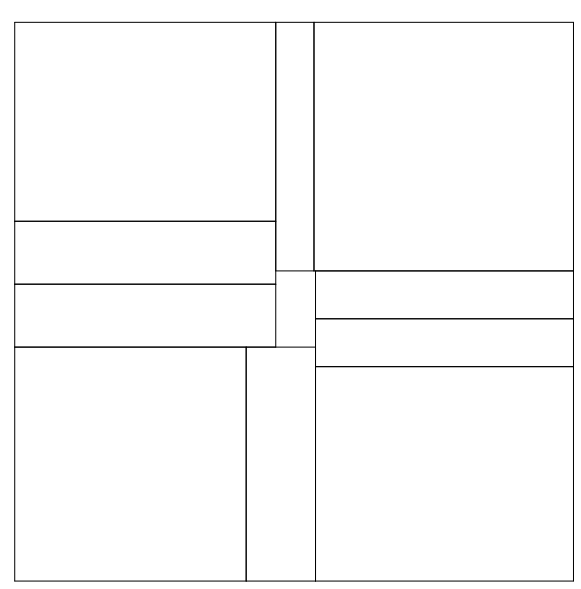

$0.35-0.5$ 


\section{ITERATION TWO}

- Steps 1 to 3 same as Iteration One

- The internal corner of each new surface is extended $50 \%$ of the distance between each adjacent surface, meeting the extrusion from the adjacent surfaces

- These new surfaces are divided evenly, or through randomised building widths, established based on minimum and maximum width values

- Through the use of logic gates some blocks are left untouched and turned into footprints, others take their divided state and also become footprints. The footprints are then scaled from their centre to create a staggered street edge 


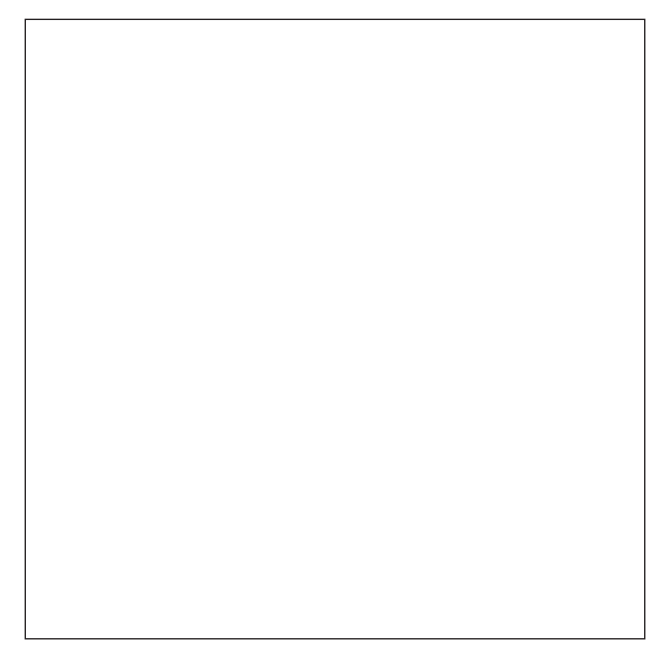

Step 01

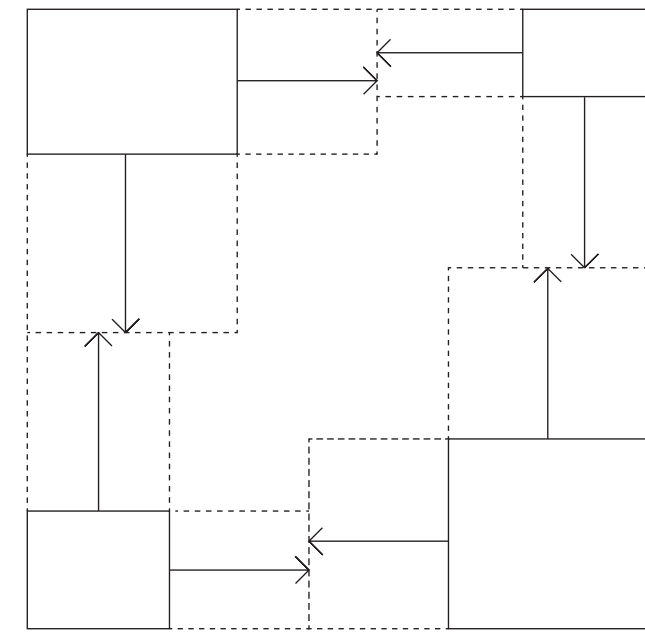

04

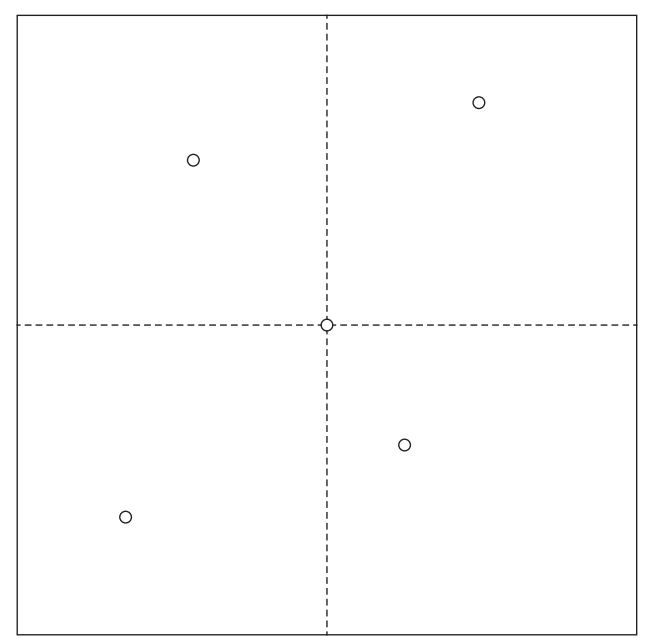

02

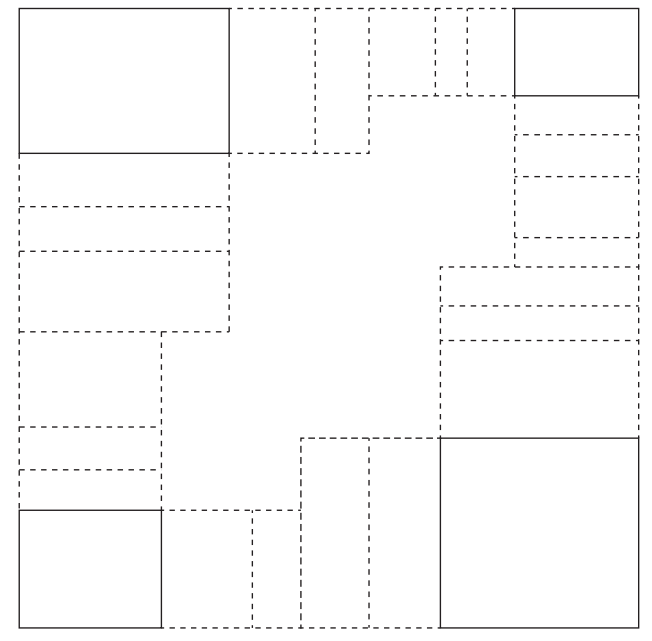

05

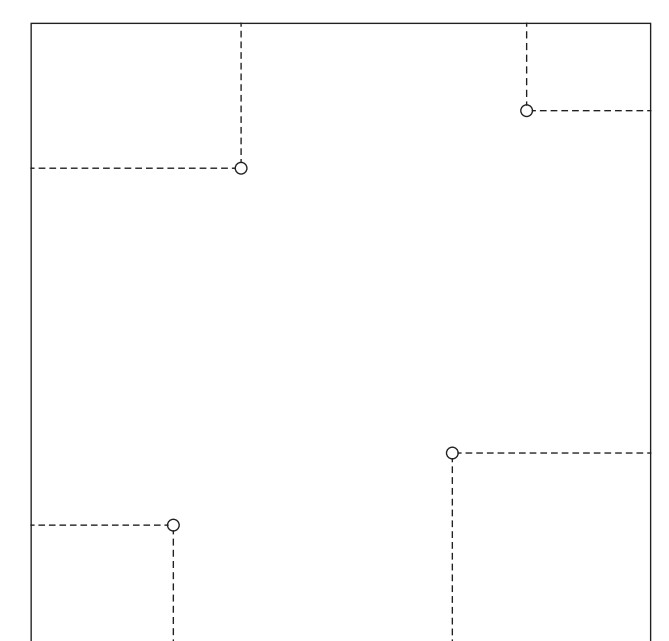

03

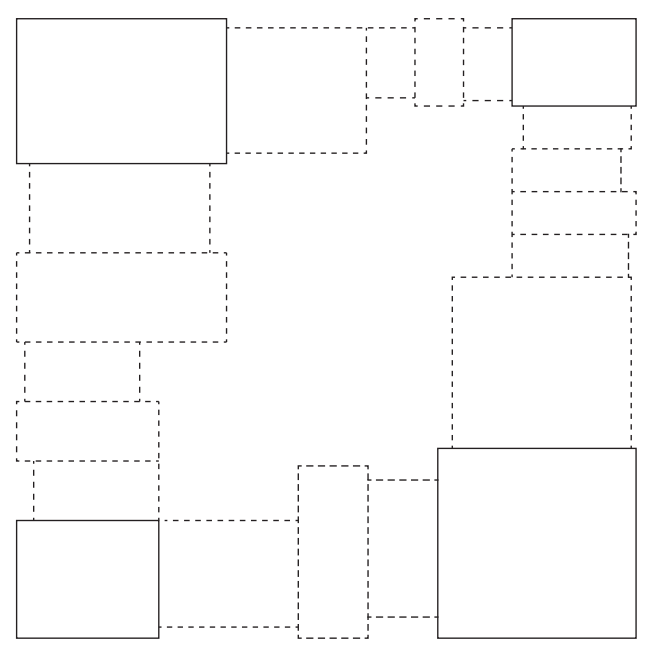

06 


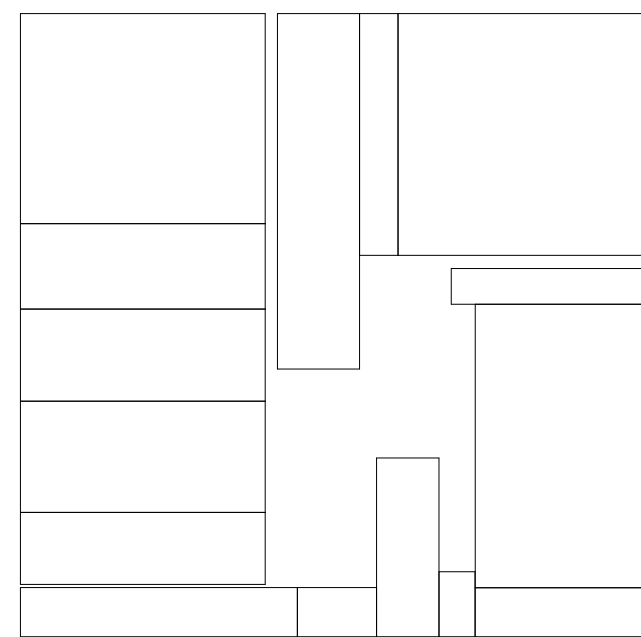

Seed 01
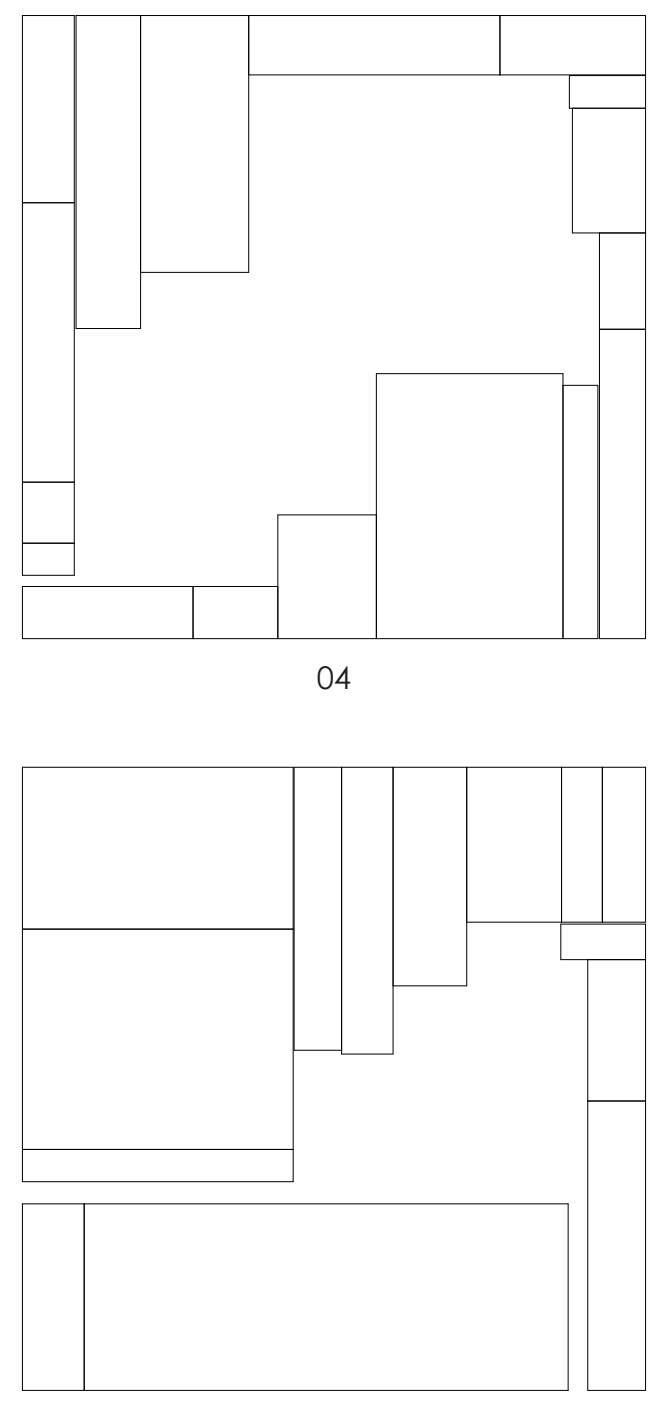

07
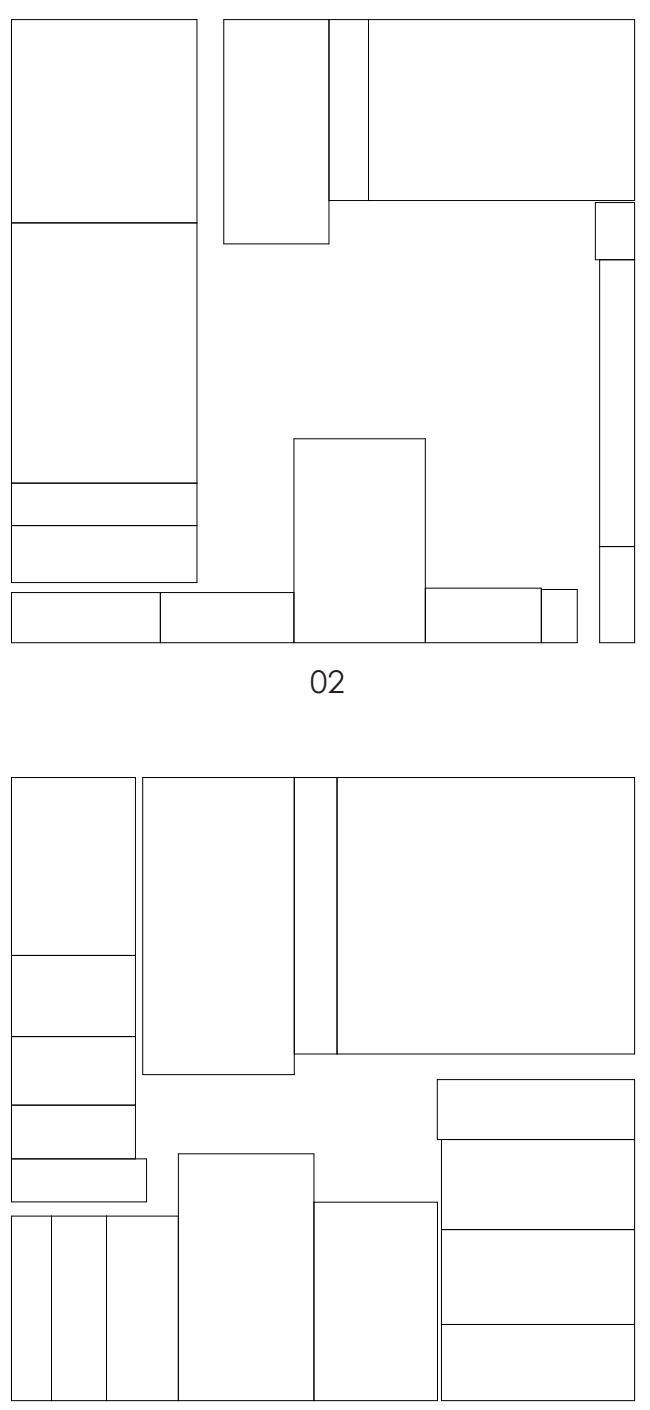

05

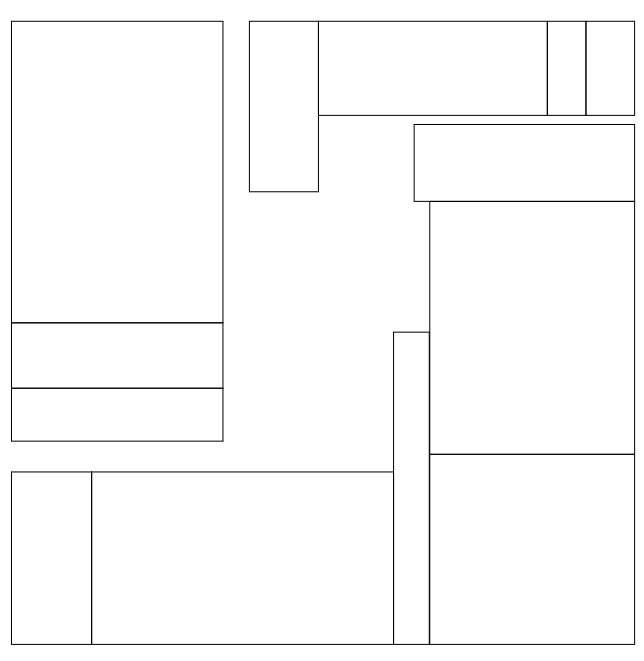

08

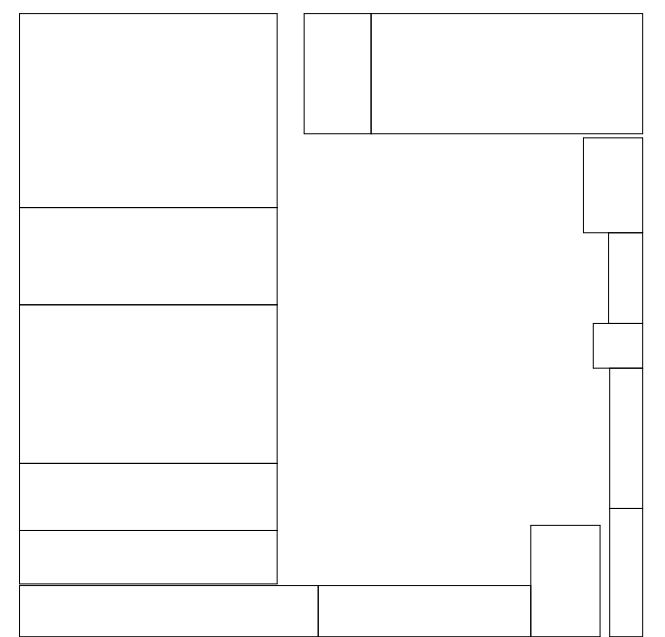

03

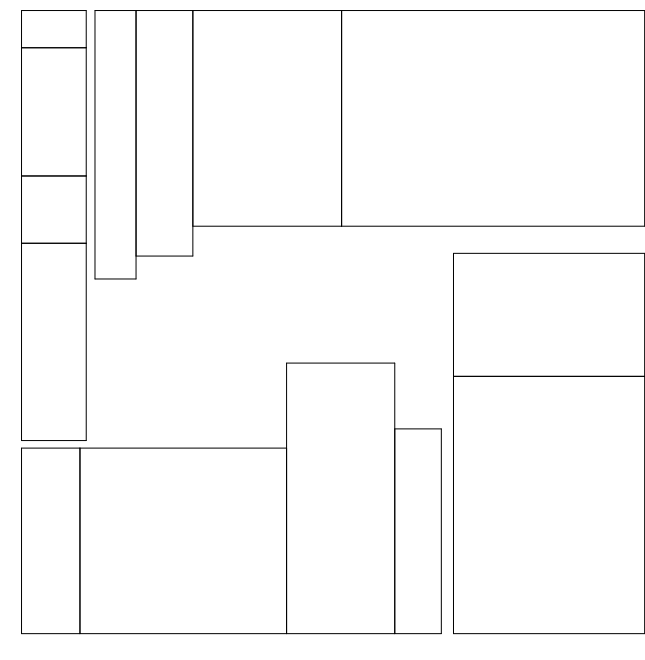

06

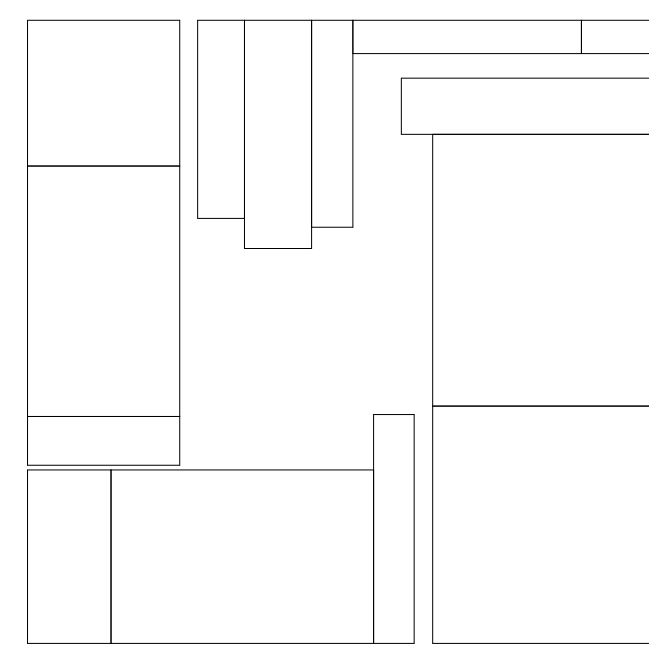

09

Figure 4.05: Compilation of Iteration Two outputs - Standard

CHAPTER FOUR

A CITY IS NOT A TREE 

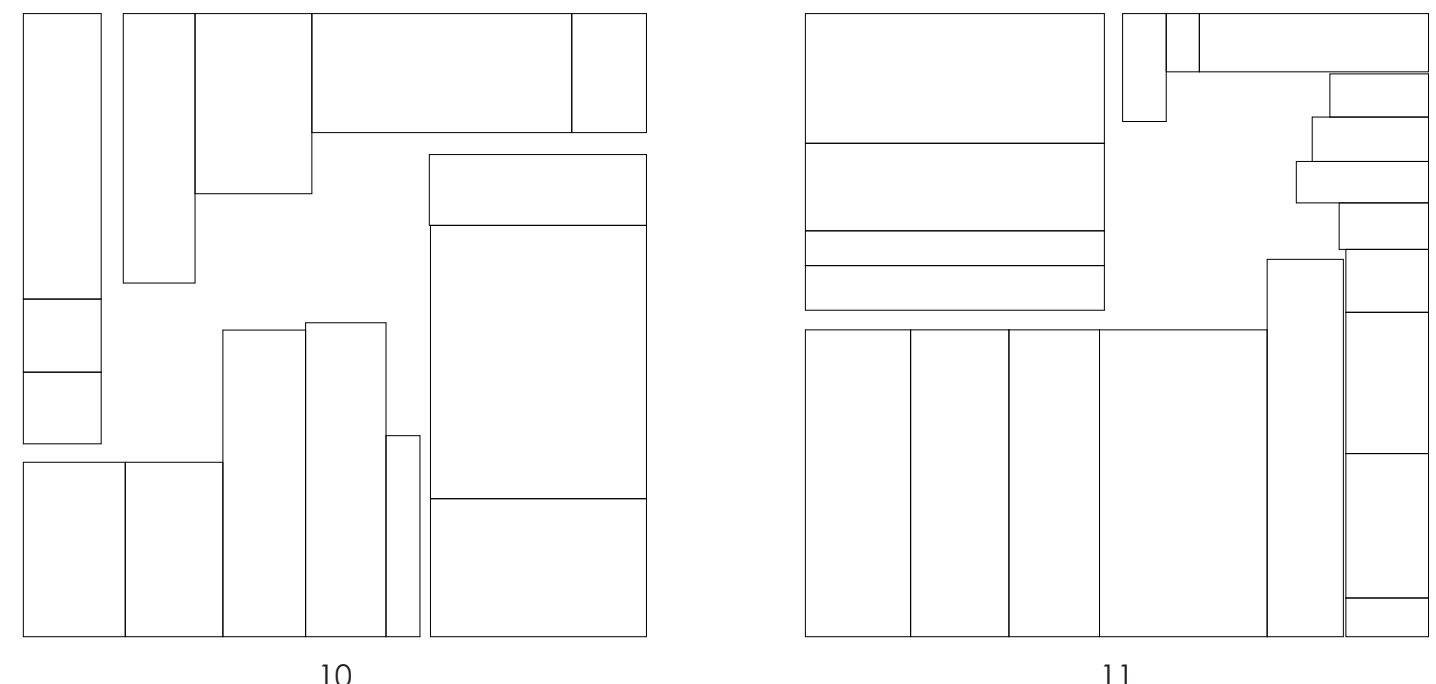

11

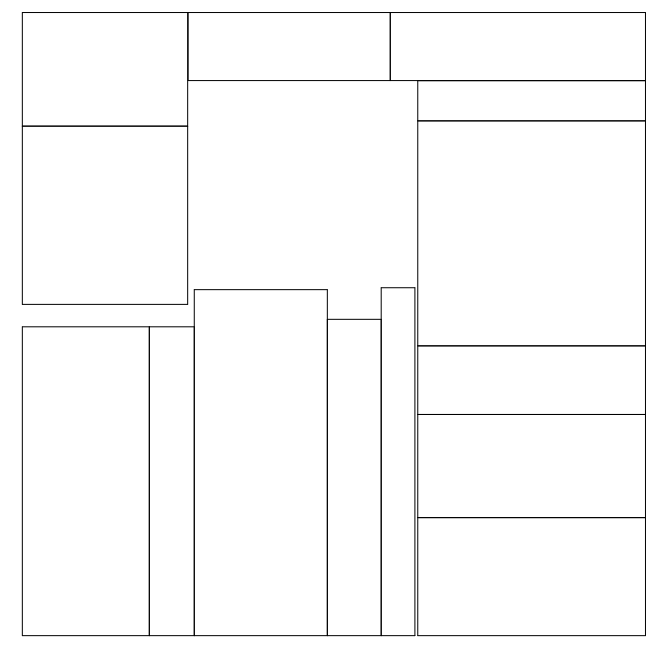

13

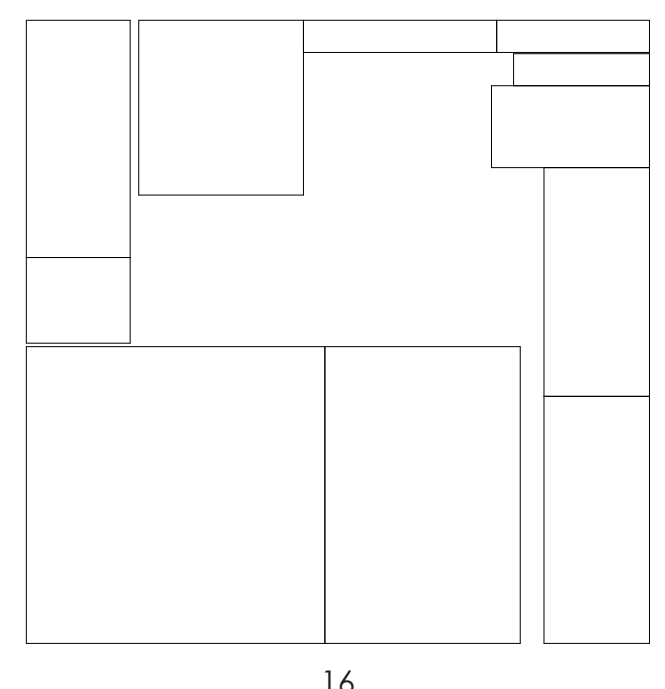

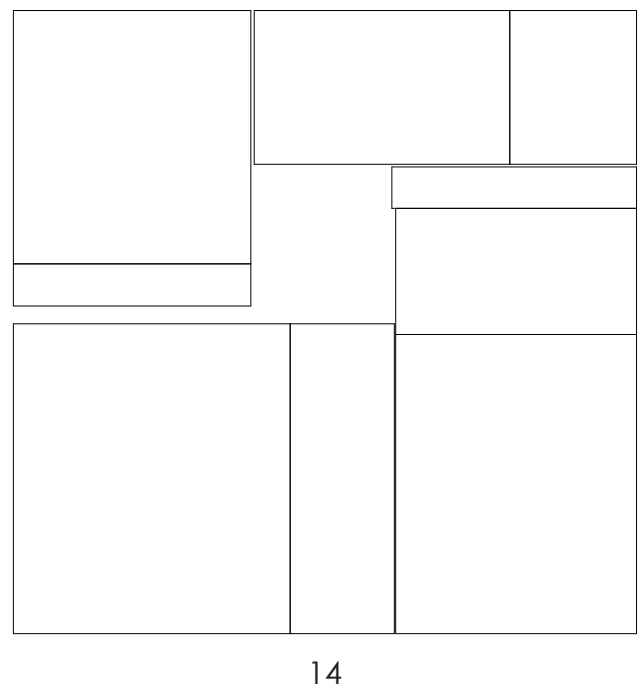

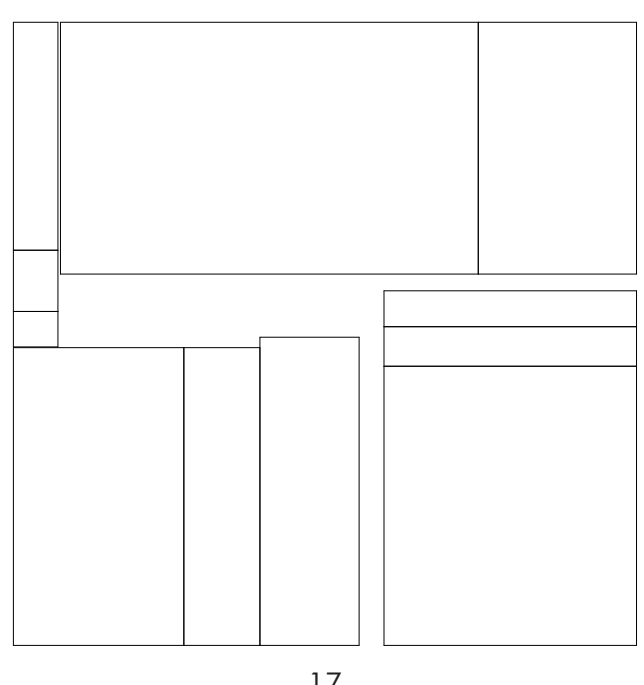

17
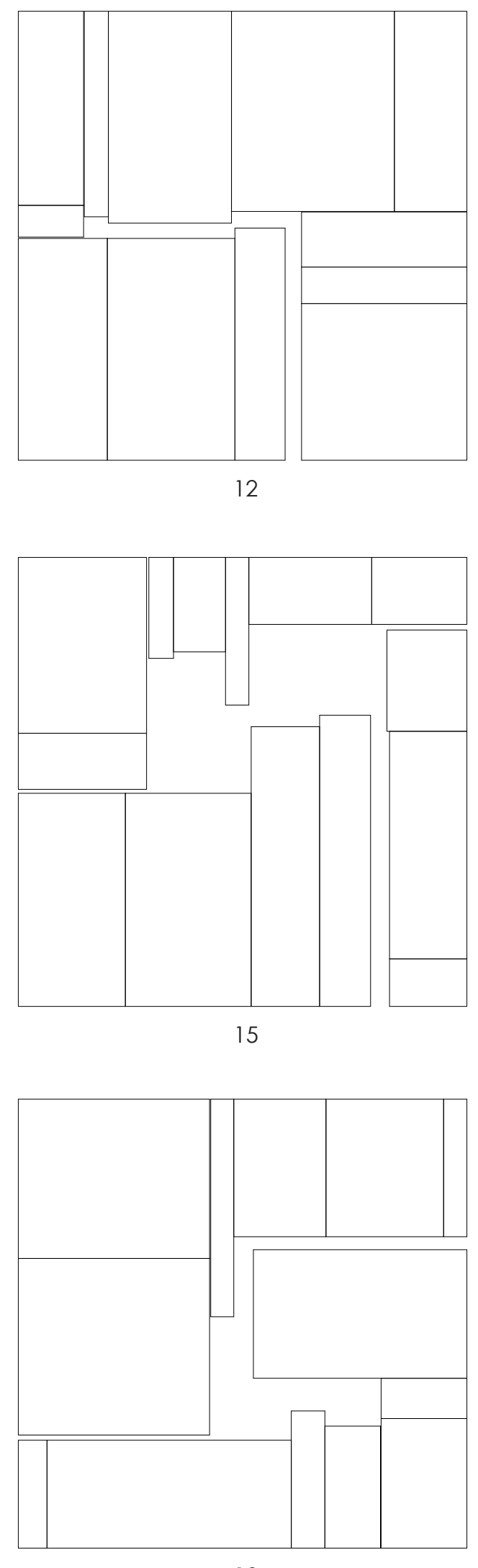

18

Figure 4.05: Compilation of Iteration Two outputs - Standard (cont.) 


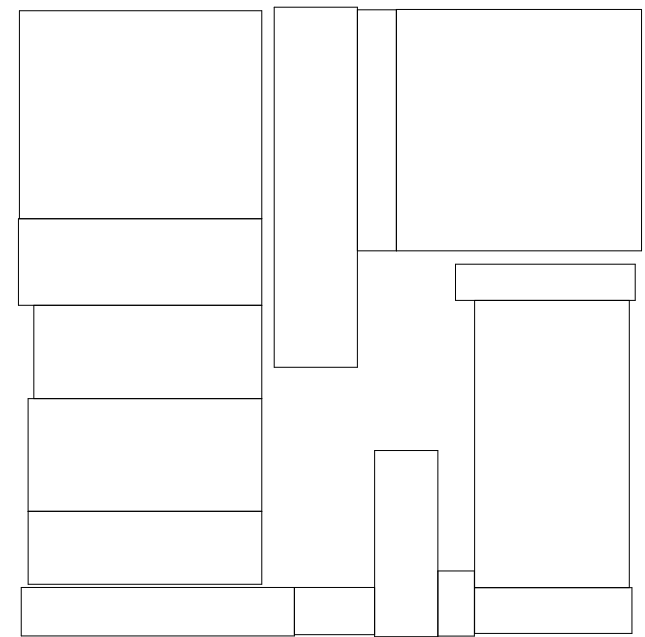

Seed 01
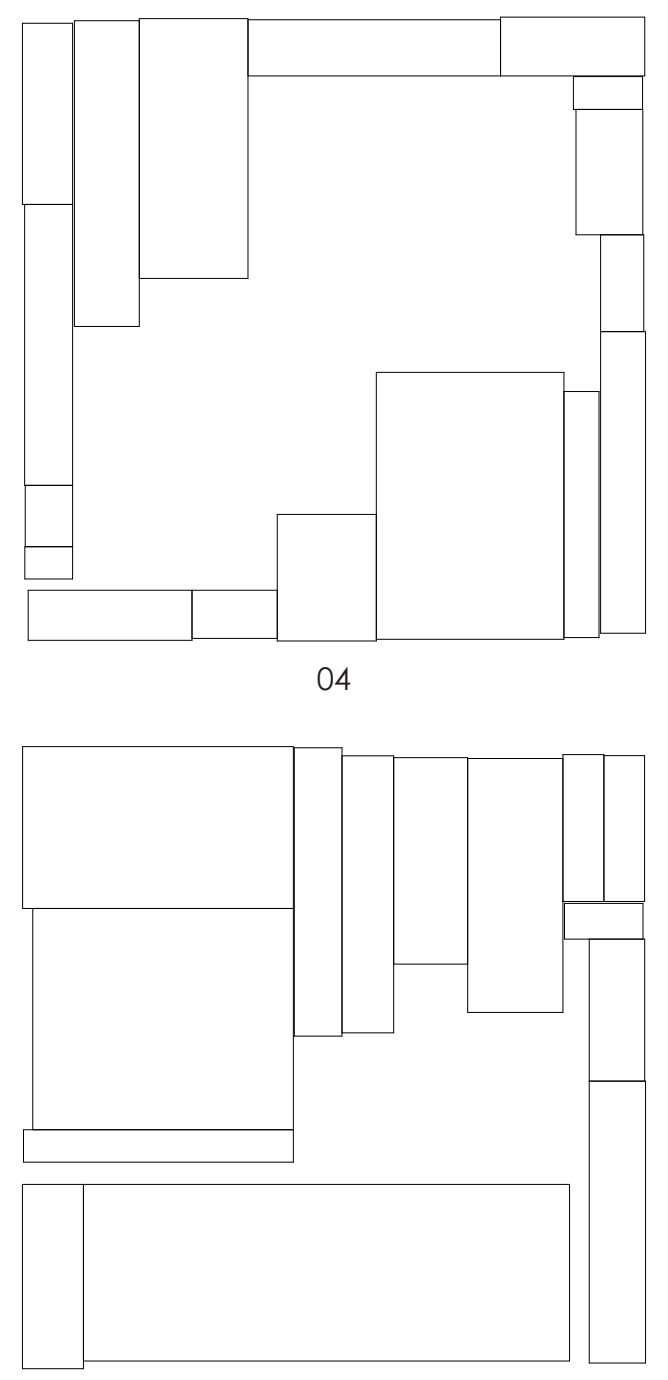

07
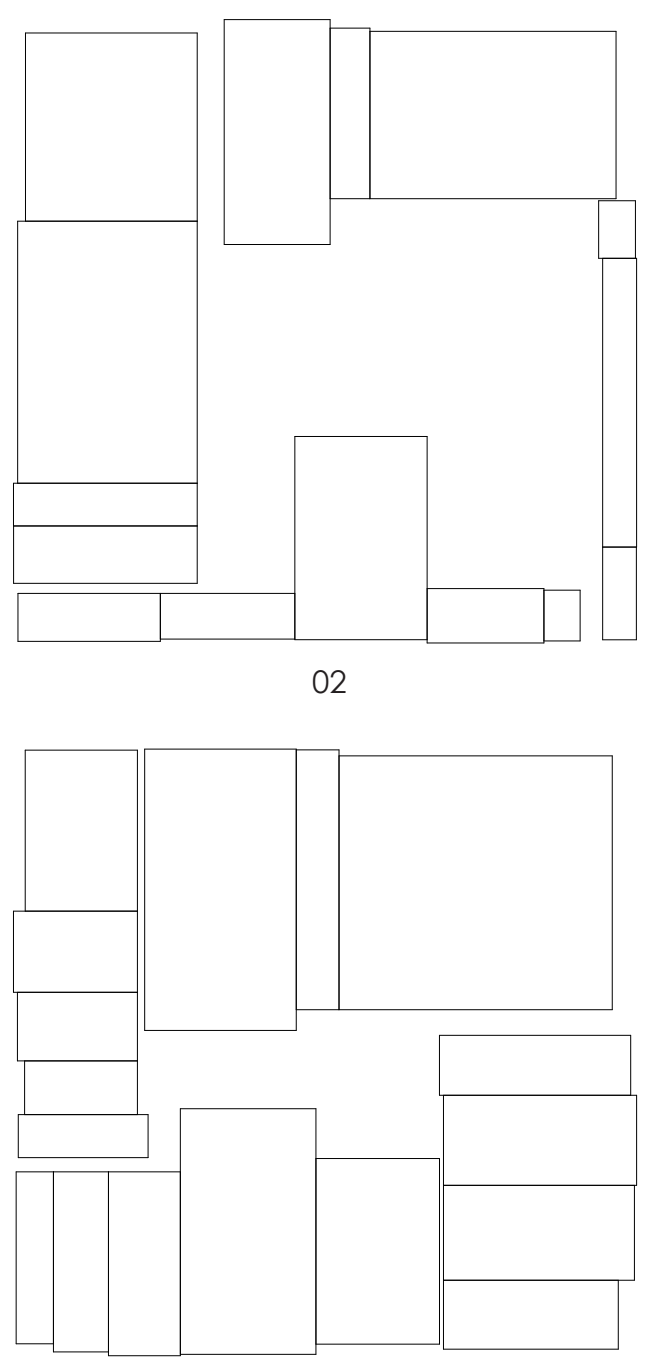

05

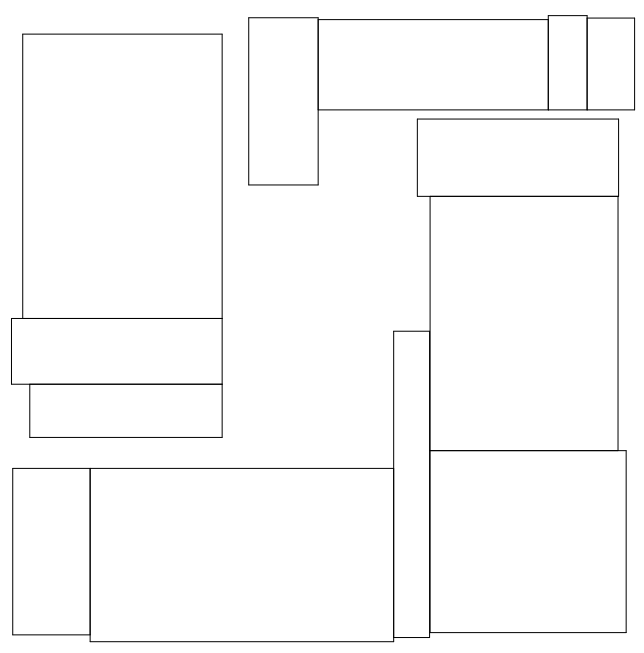

08

Figure 4.06: Compilation of Iteration Two outputs

CHAPTER FOUR

\section{A CITY IS NOT A TREE \\ ACIYIS NOTATREE}

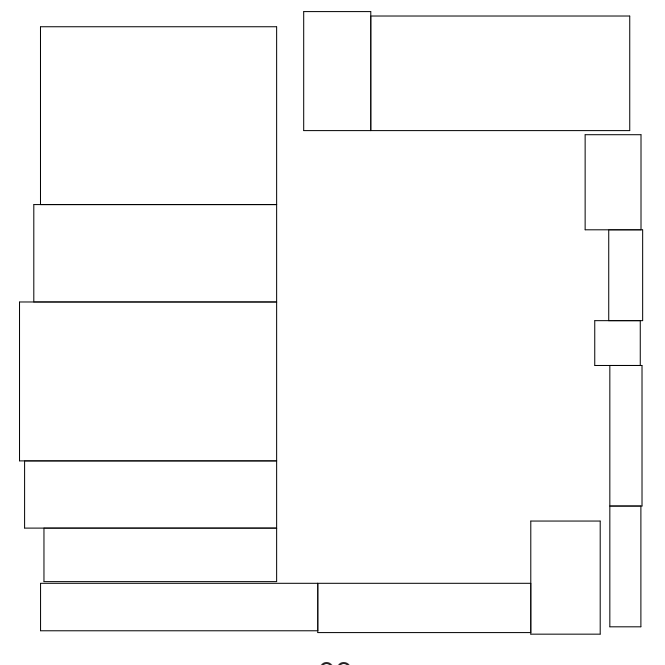

03

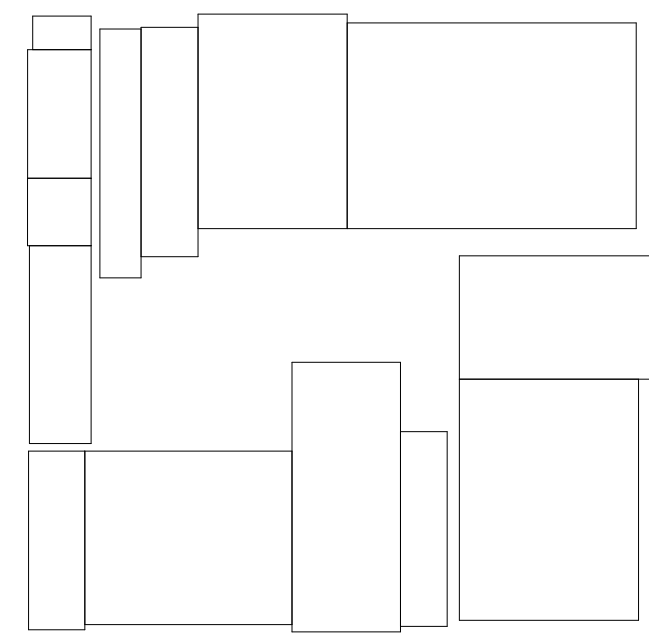

06

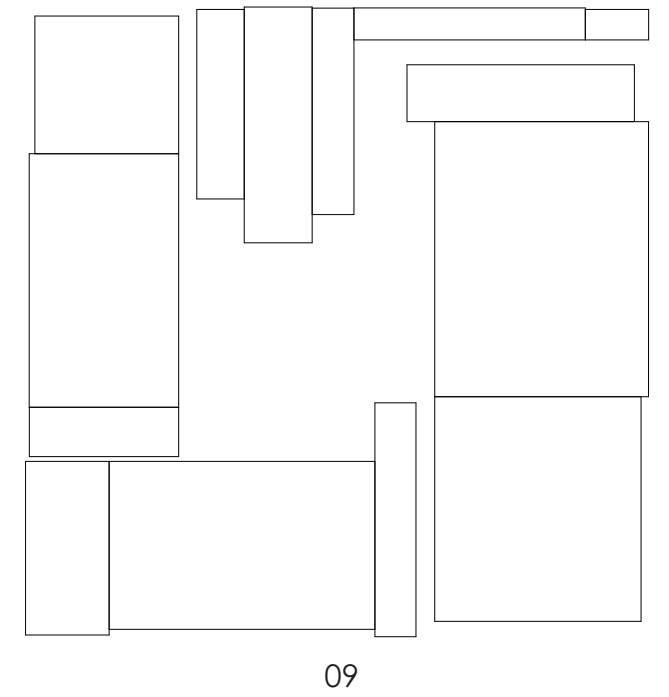

Base jittering

$0.9-1.0$ 


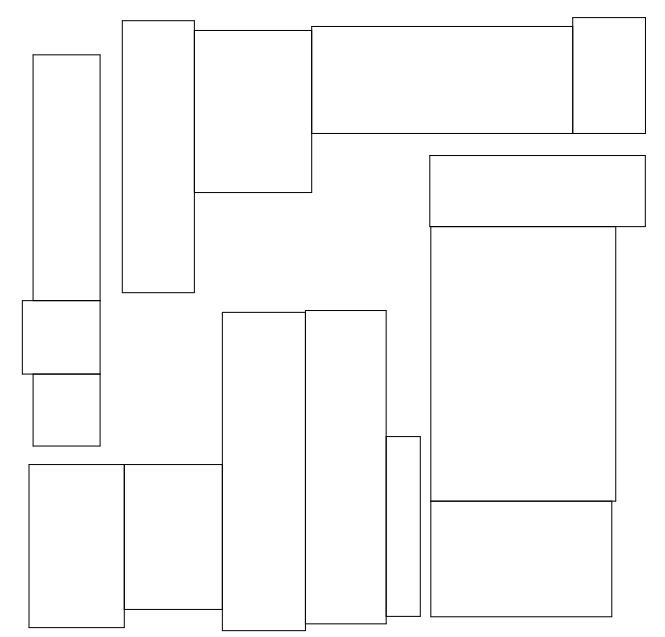

Seed 10

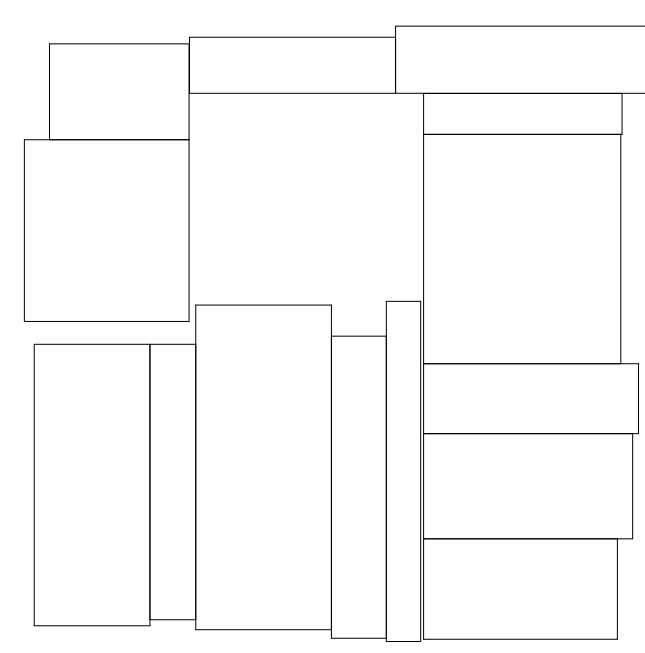

13

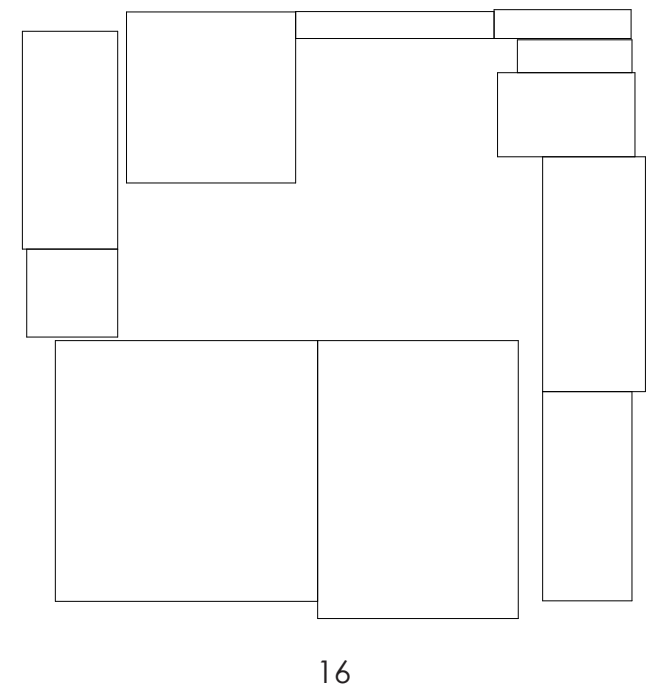

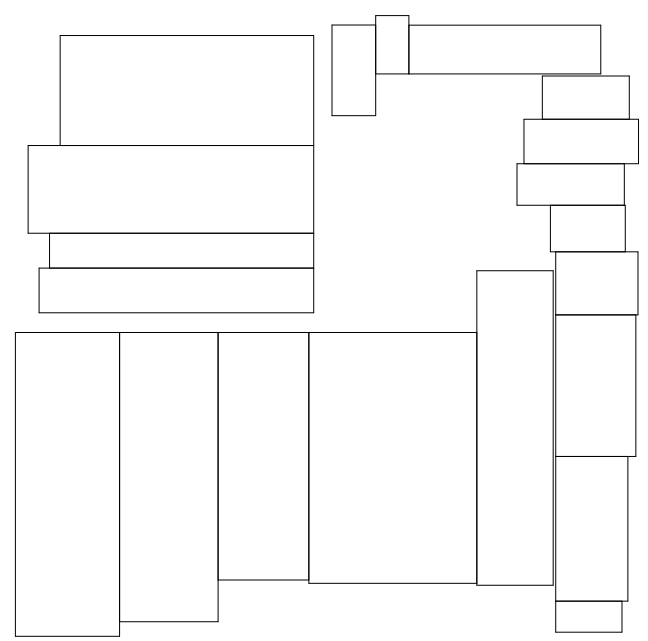

11

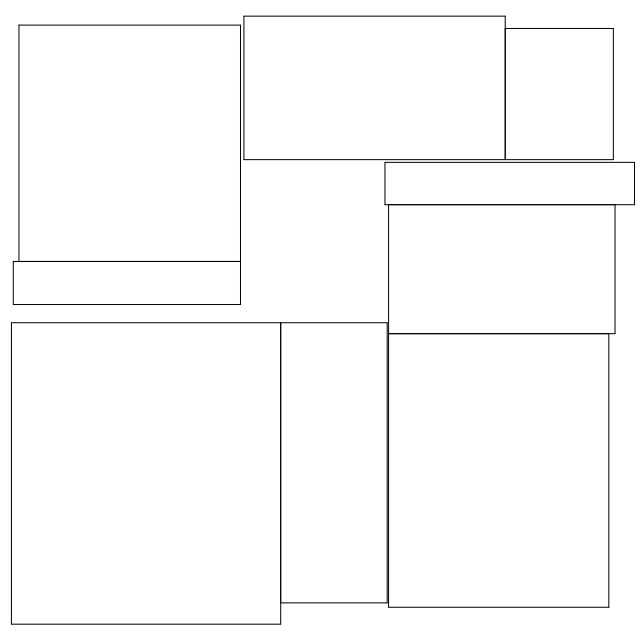

14

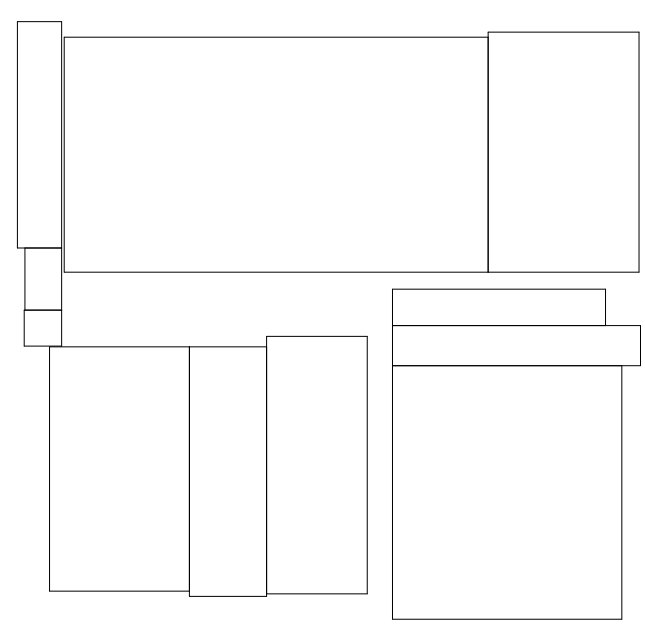

17

Figure 4.07: Compilation of Iteration Two outputs

page 056

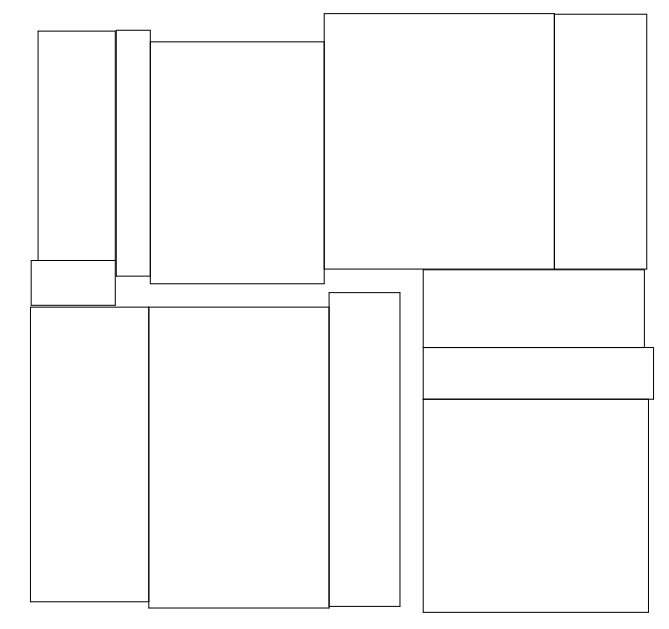

12

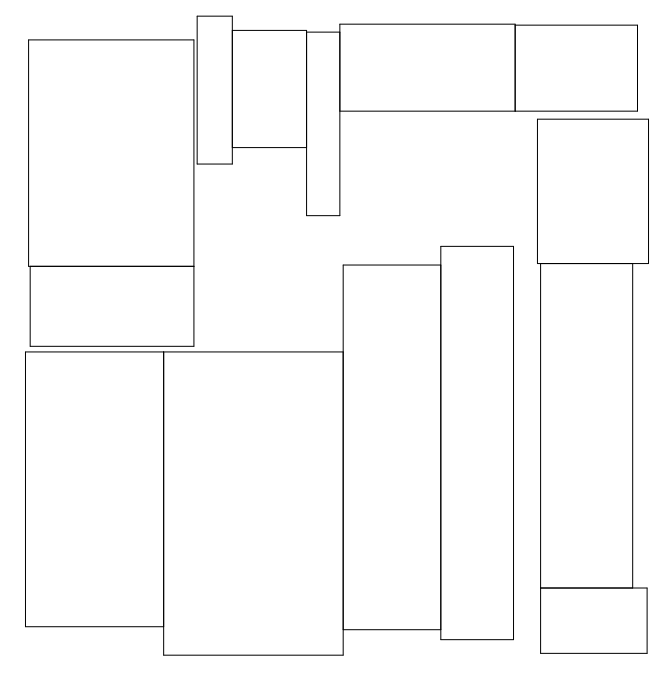

15

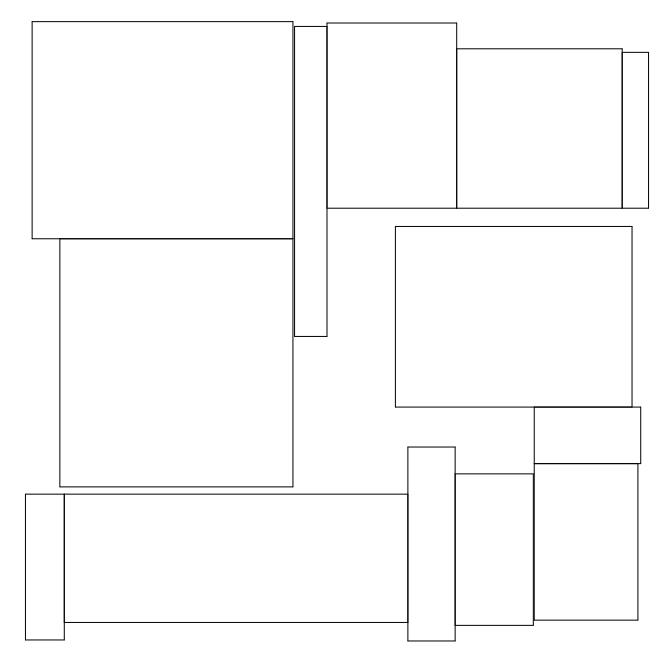

18

Base jittering

$0.8-1.0$

PAGE0S6 


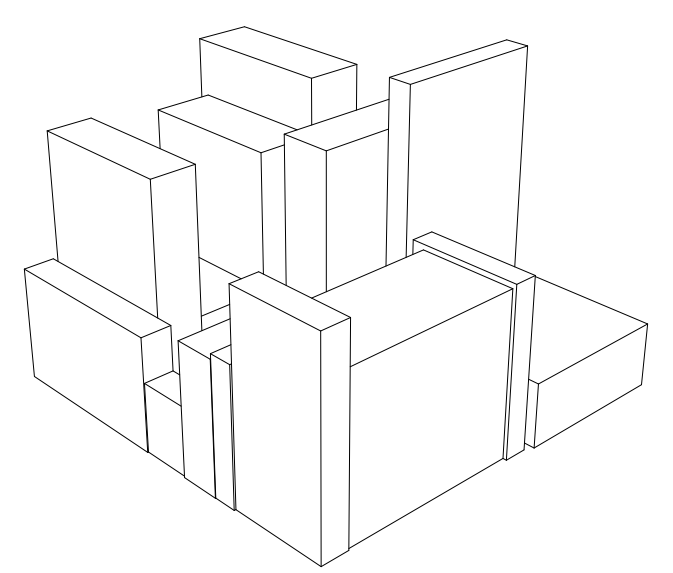

Seed 01

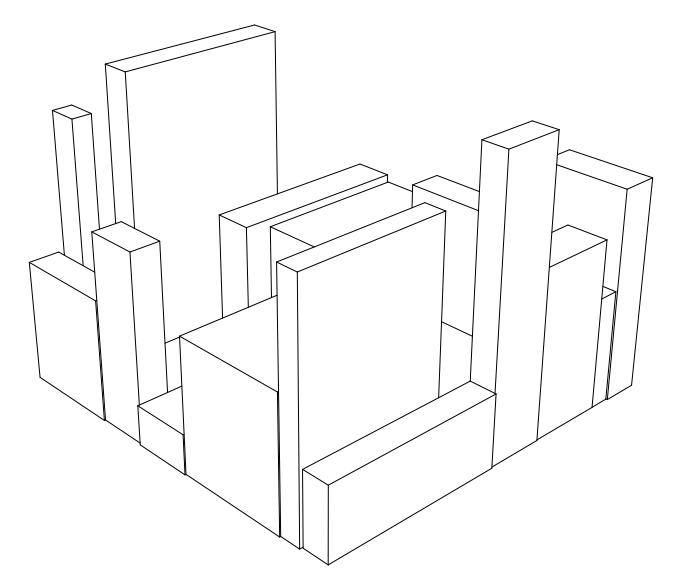

04

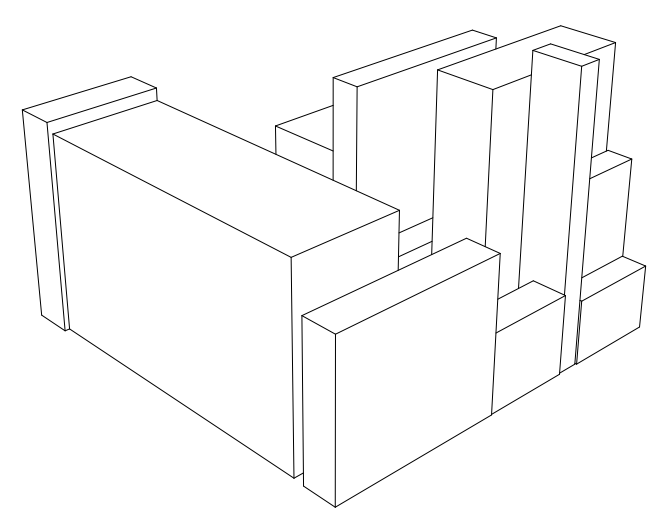

07

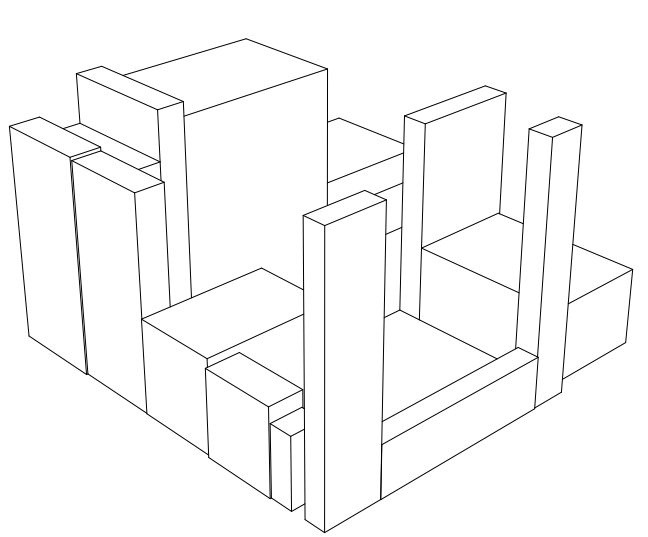

02

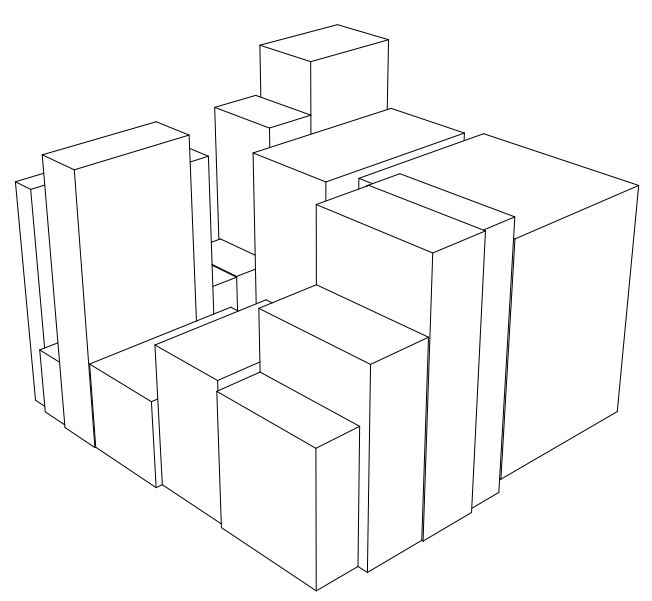

05

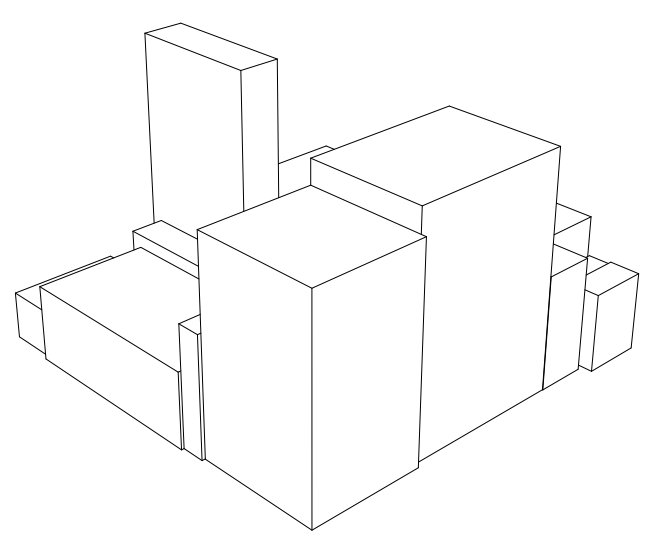

08

Figure 4.08: Compilation of Iteration Two outputs

CHAPTER FOUR

A CITY IS NOT A TREE

A CITY IS

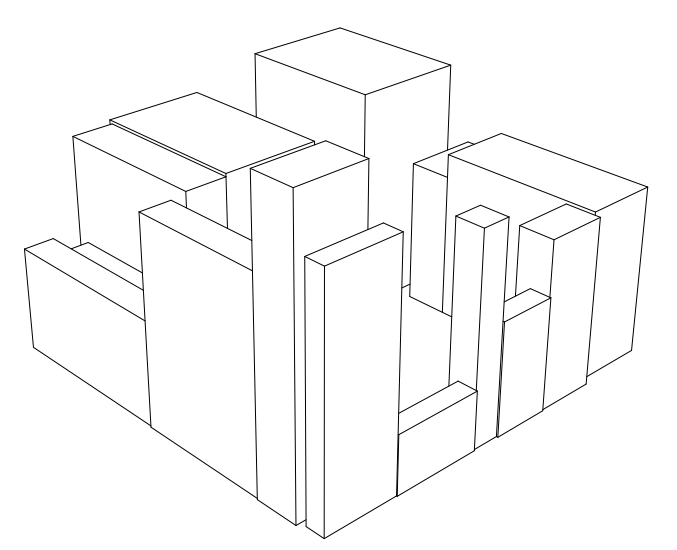

03

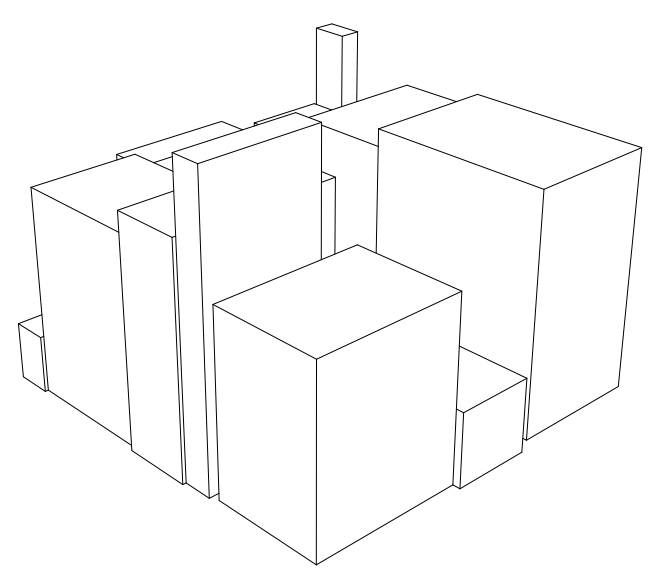

06

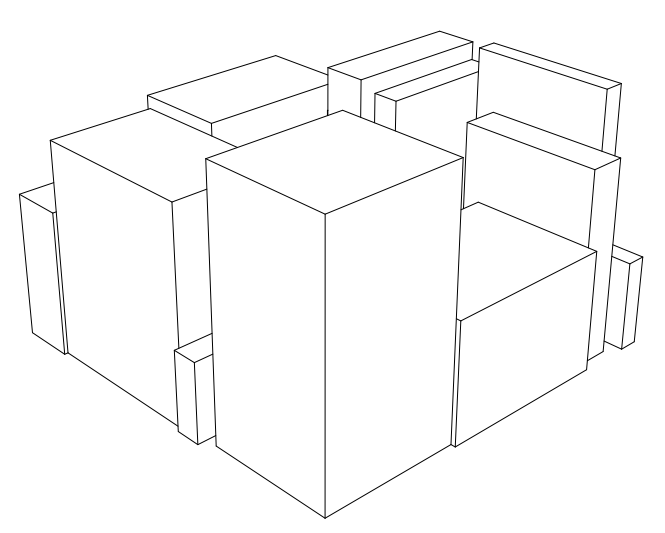

09

\begin{tabular}{|l|r|}
\hline Base jittering & $0.9-1.0$ \\
\hline Extrusion & $10-70 \mathrm{~m}$ \\
\hline
\end{tabular}




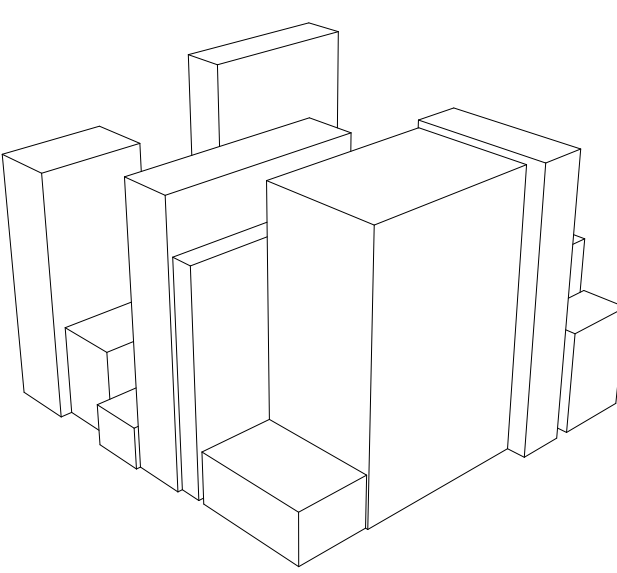

Seed 10

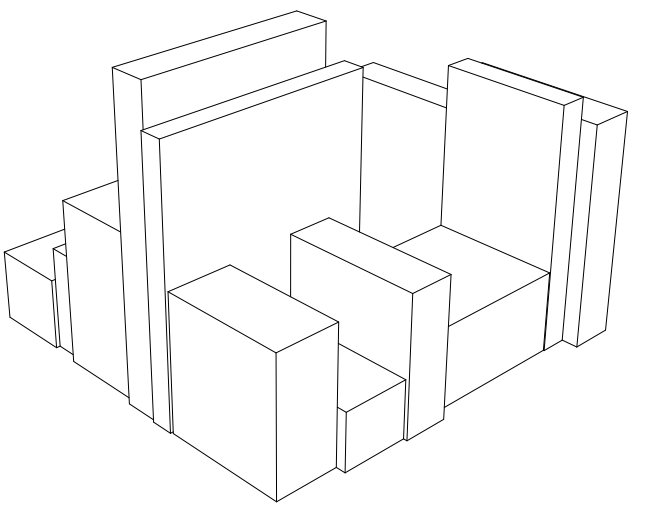

13

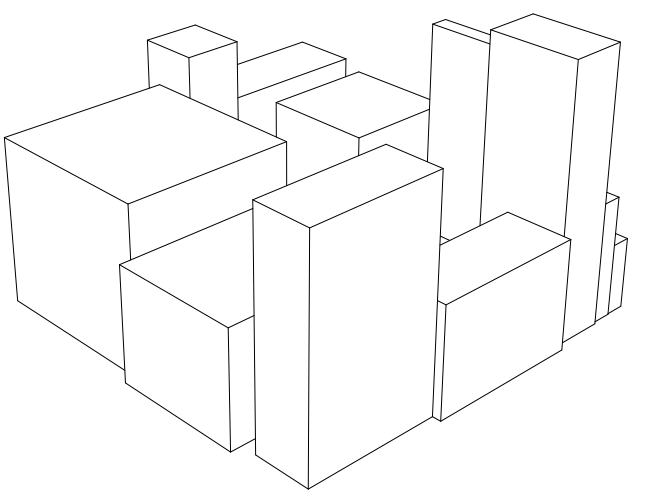

16

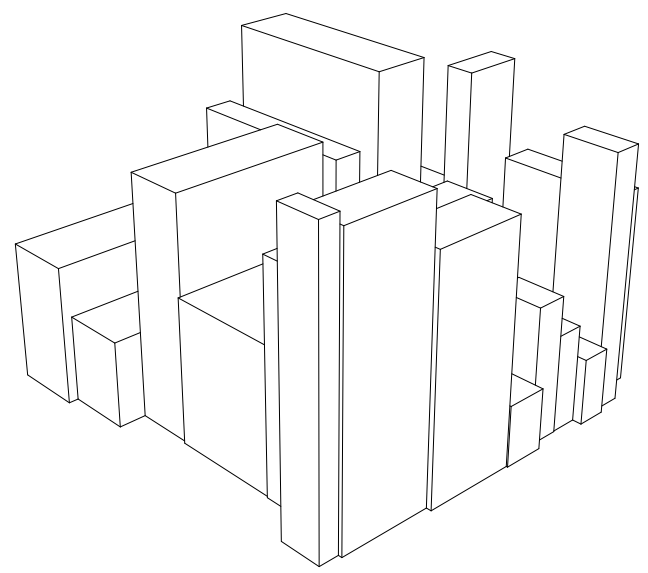

11

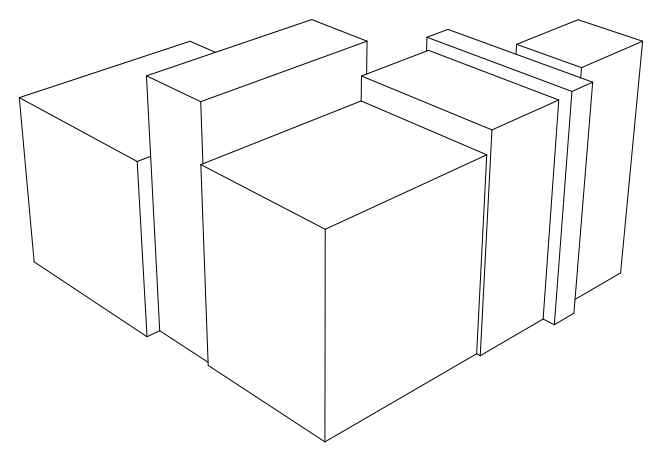

14

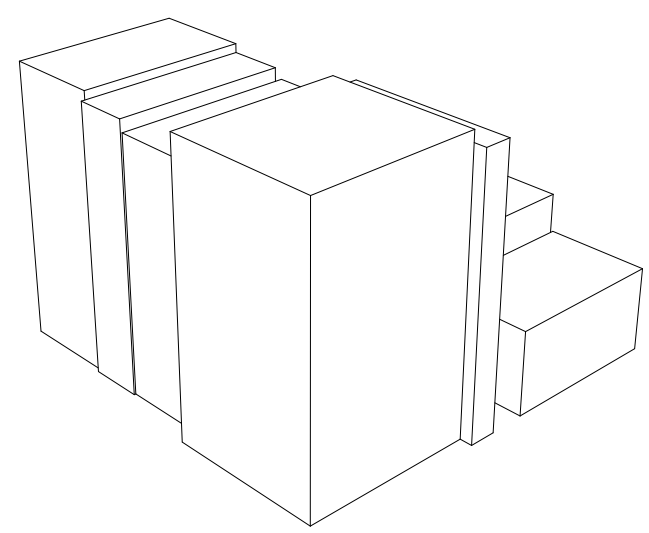

17

Figure 4.09: Compilation of Iteration Two outputs PAGE 058

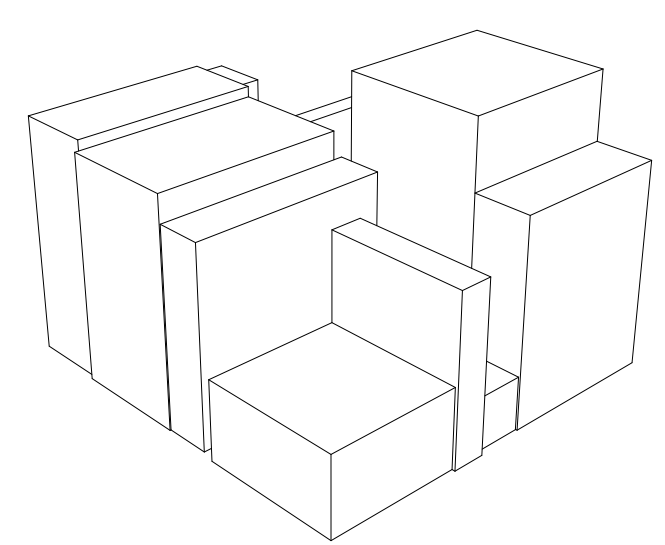

12

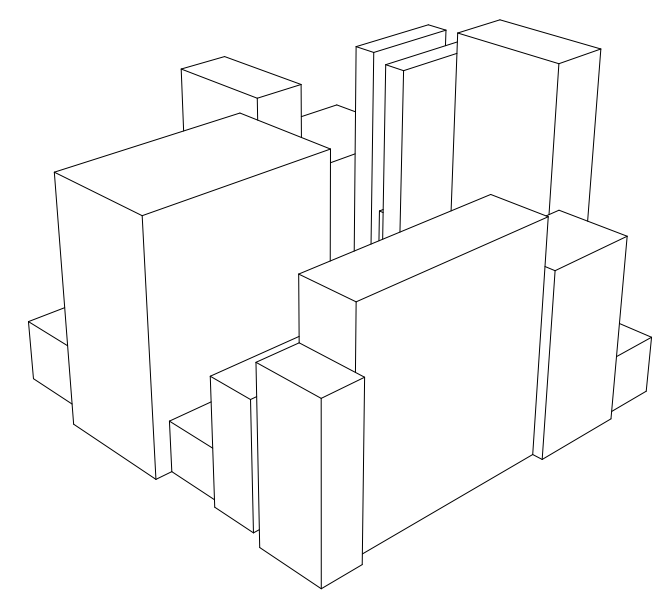

15

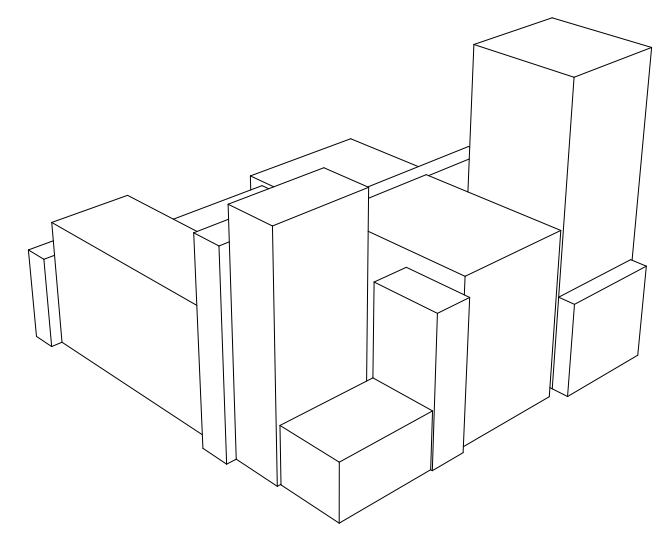

18

\begin{tabular}{|l|r|}
\hline Base jittering & $0.8-1.0$ \\
\hline Extrusion & $10-70 \mathrm{~m}$ \\
\hline
\end{tabular}

per 


\section{ITERATION THREE}

- Steps 1 to 4 same as Iteration One

- Using the surface that is generated in the centre of the original surface another point is randomly placed. This point is then used to divide the internal surface into four new surfaces

- The four new surfaces are joined to the four existing infill surfaces through a region union, creating four new six-sided geometries

- Six sided geometries are divided based on a minimum/ maximum width range and RNG to establish building footprints

- Each footprint is scaled from its centre to create a staggered street edge

Iteration Three had one major issue that meant it was inappropriate to work with. The first of those issues is that the system would inconsistently generate four or six sided geometries, depending on the division of the larger six-sided geometries. This caused several issues when trying to establish processes to create facade geometries, amongst other problems. 


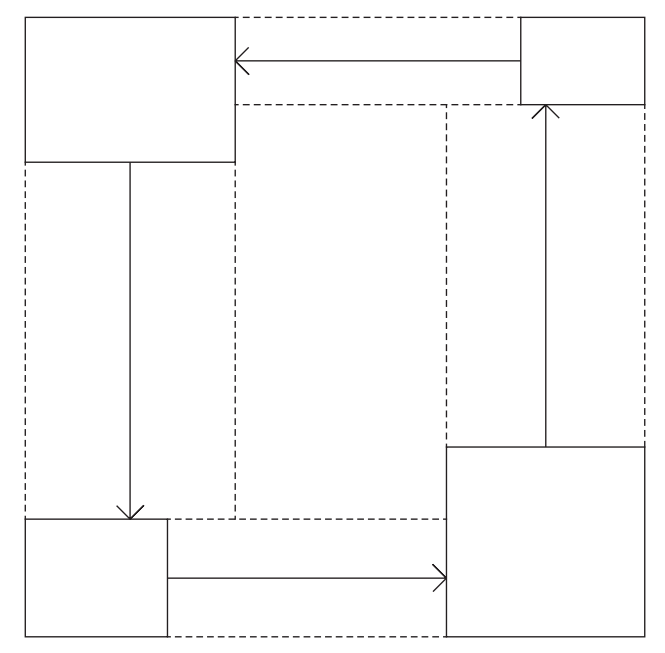

Step 01

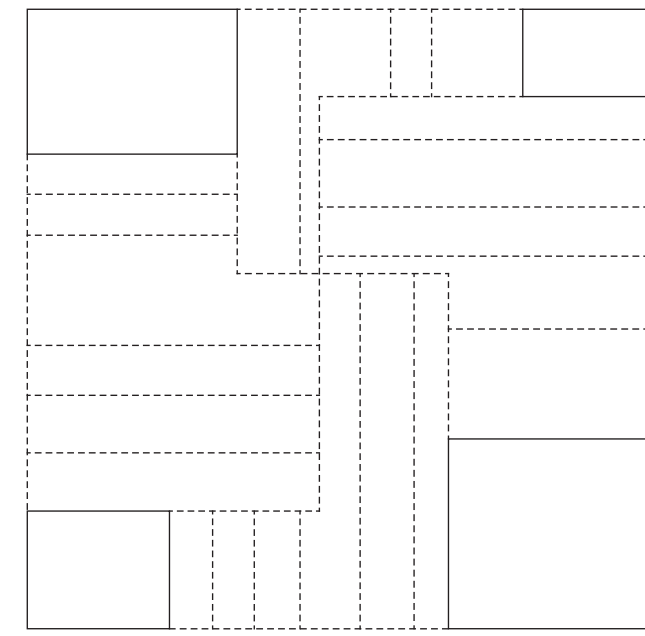

04

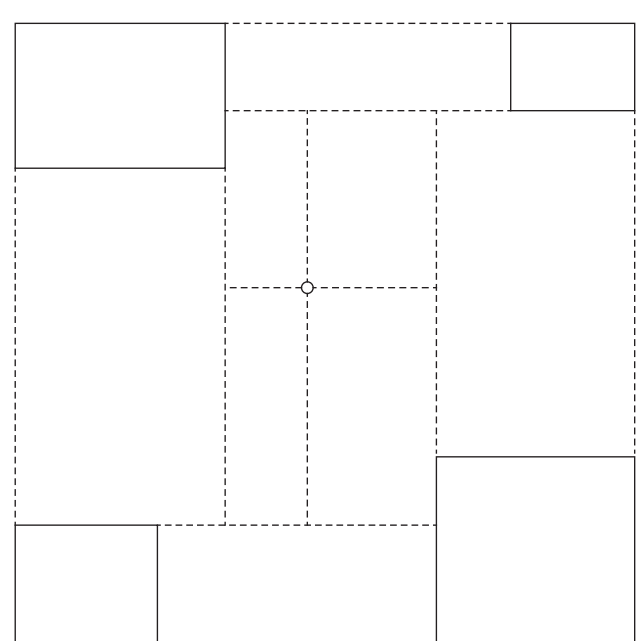

02

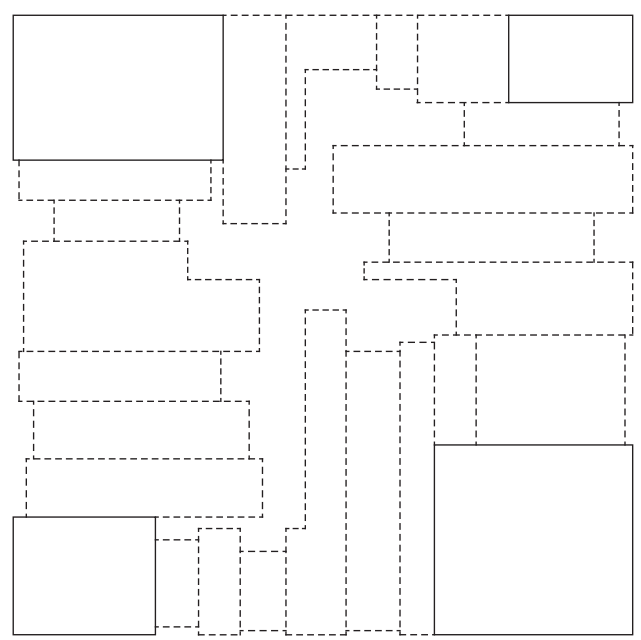

05

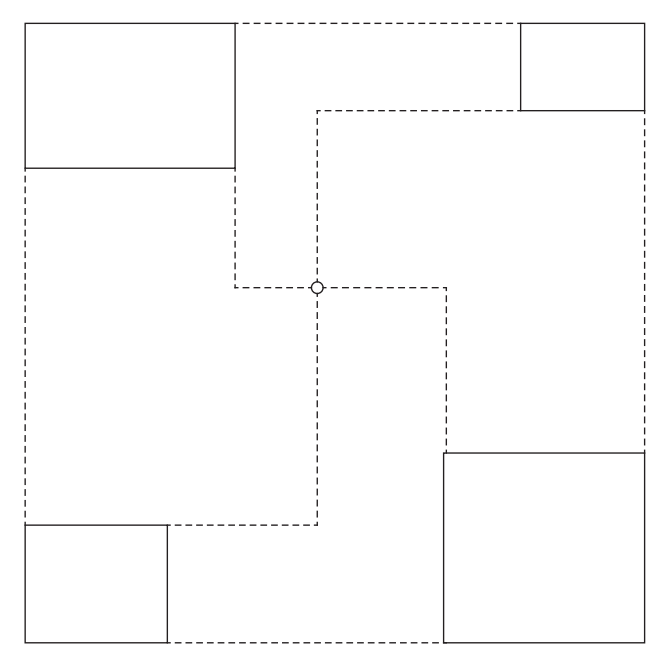

03

Figure 4.10: Construction logic for creating Iteration Three blocks 


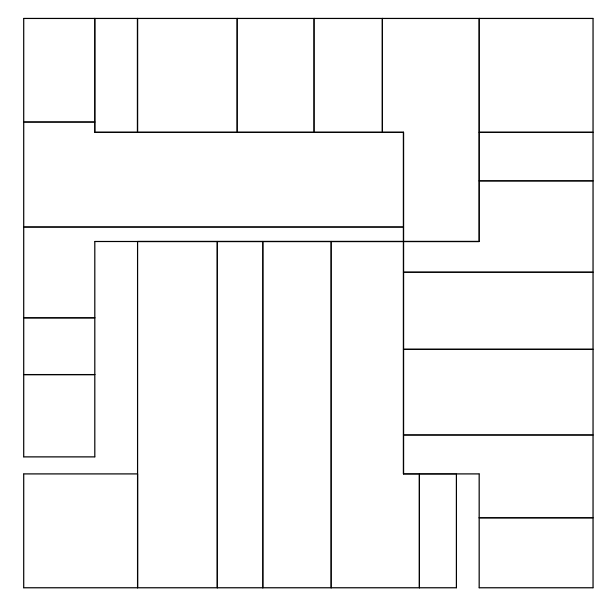

Seed 00

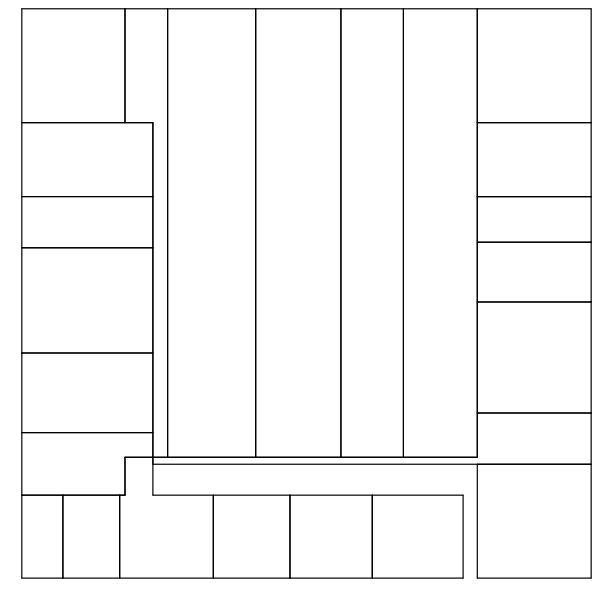

04

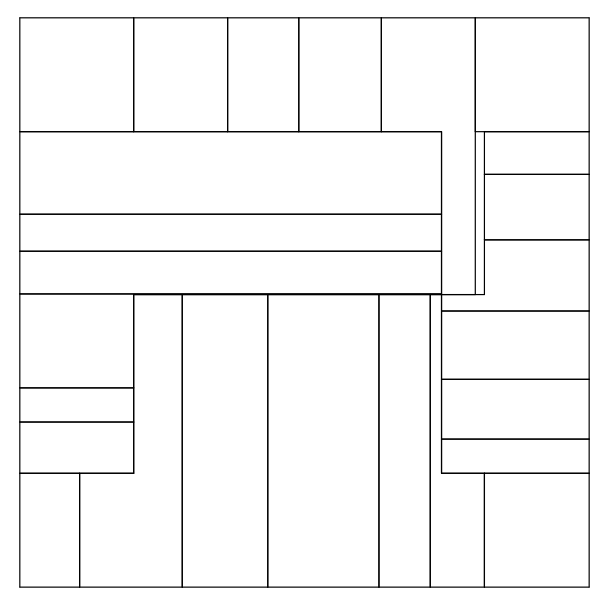

08

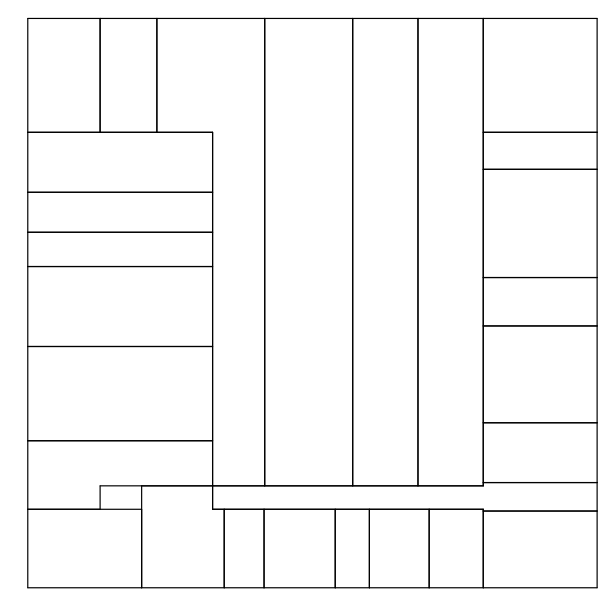

01

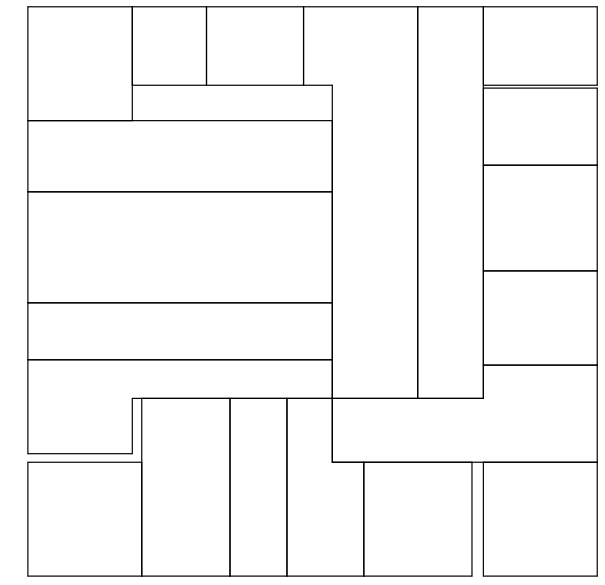

05

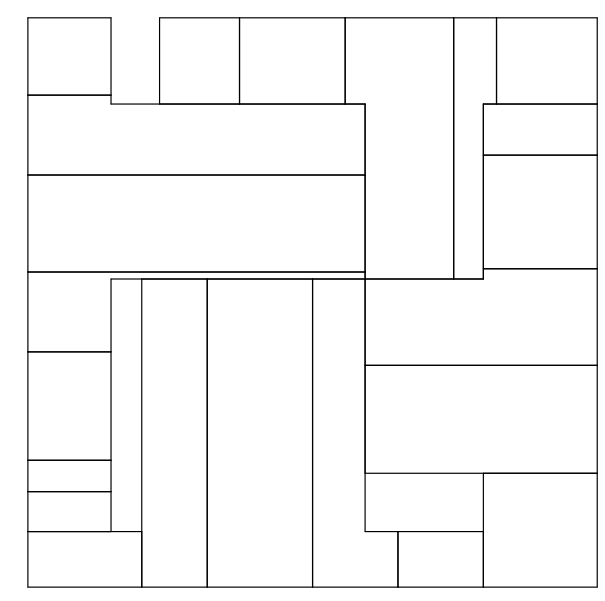

09

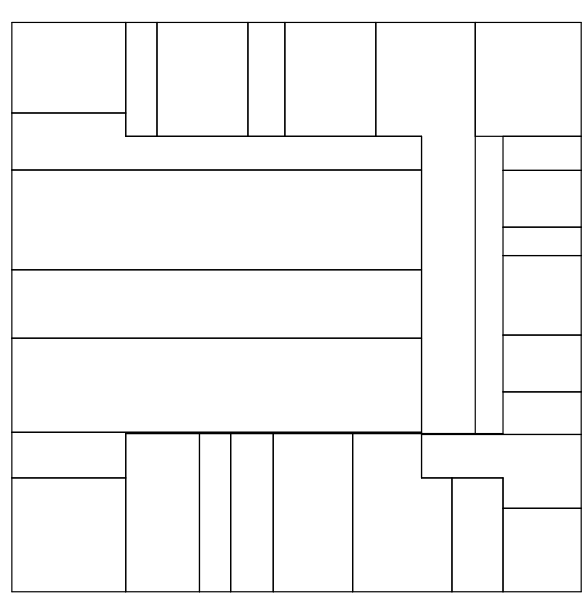

02

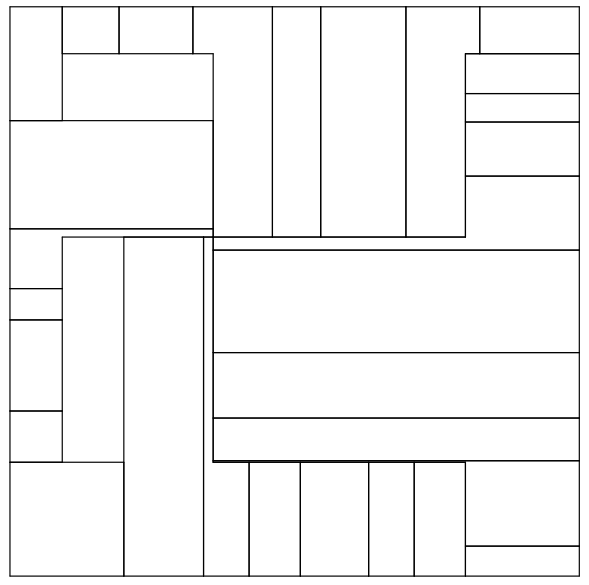

06

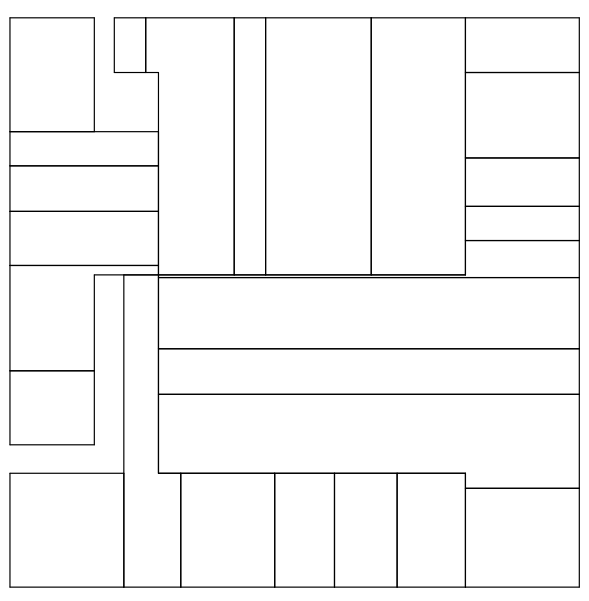

10

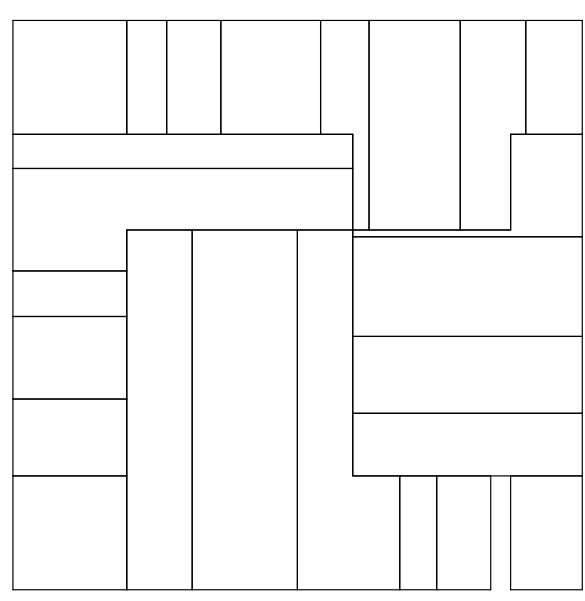

03

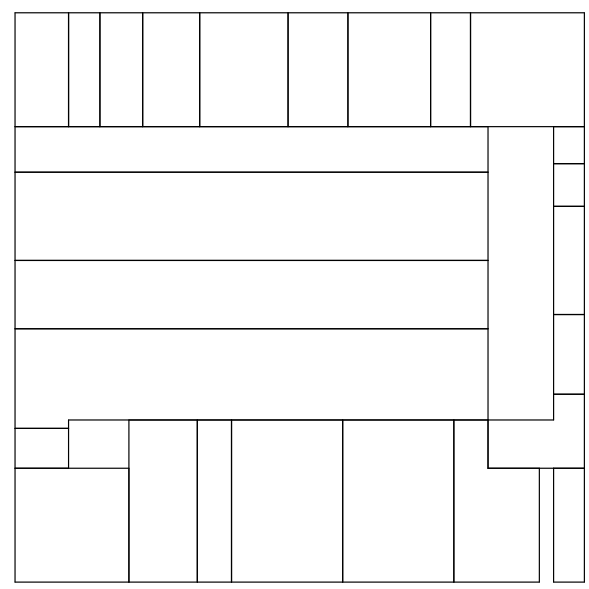

07

\begin{tabular}{|l|r|}
\hline Base Surface Size & $200 \times 200$ metres \\
\hline Minimum Building Widths & $10.0 \mathrm{~m}$ \\
\hline Maximum Building Widths & $40.0 \mathrm{~m}$ \\
\hline
\end{tabular}

Figure 4.11: Compilation of outputs - Standard version

CHAPTER FOUR

A CITY IS NOT A TREE 


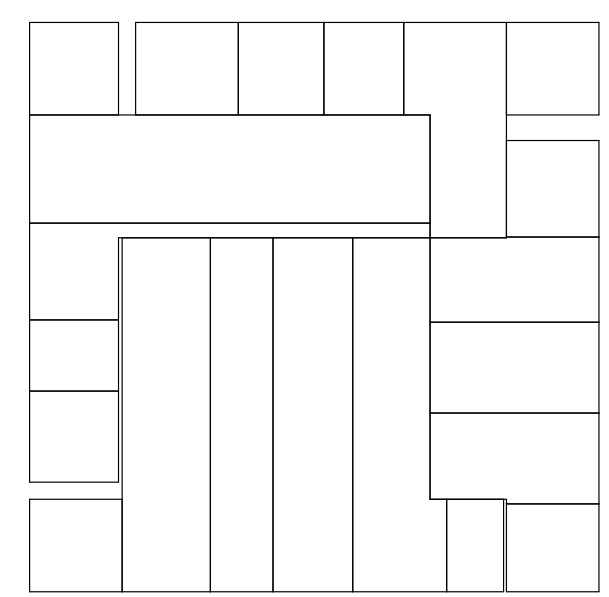

Seed 00

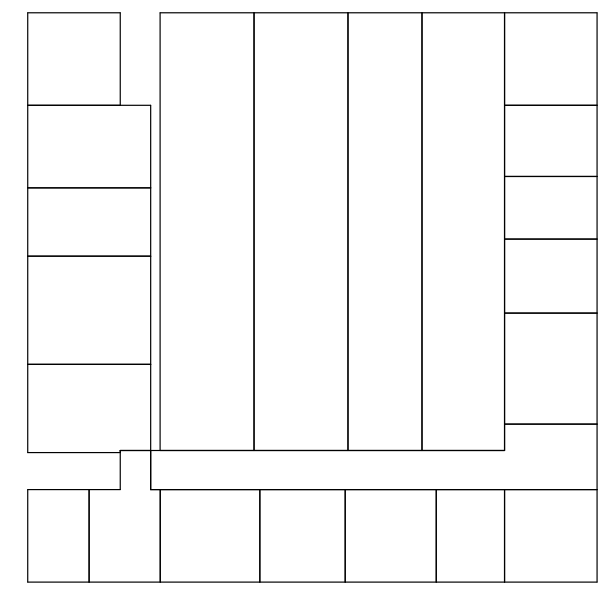

04

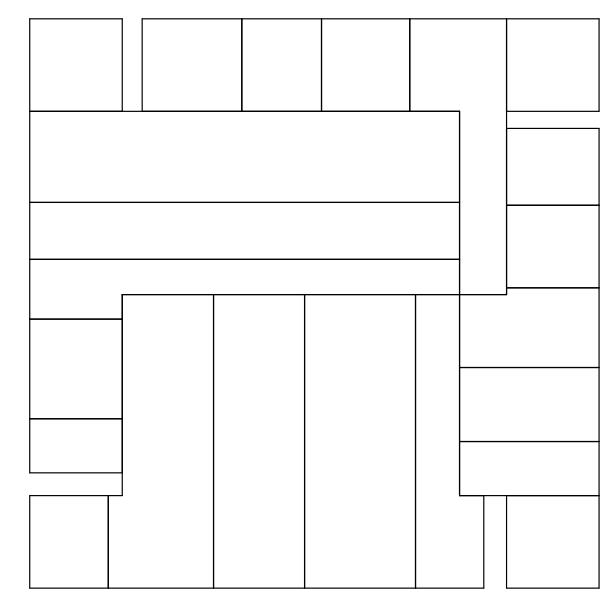

08

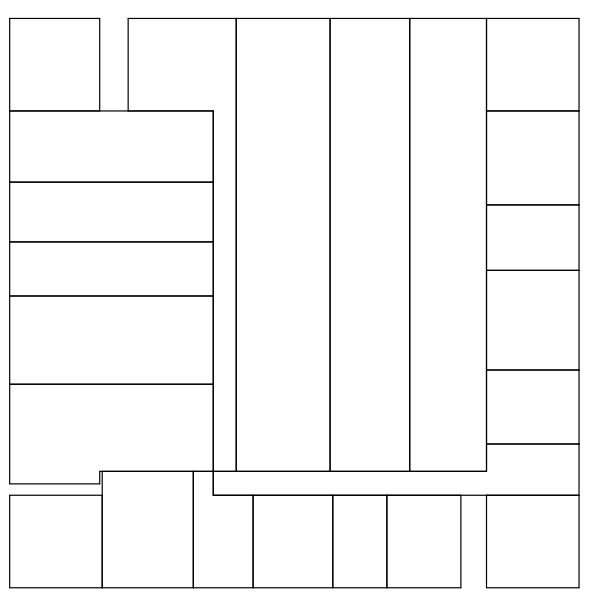

01

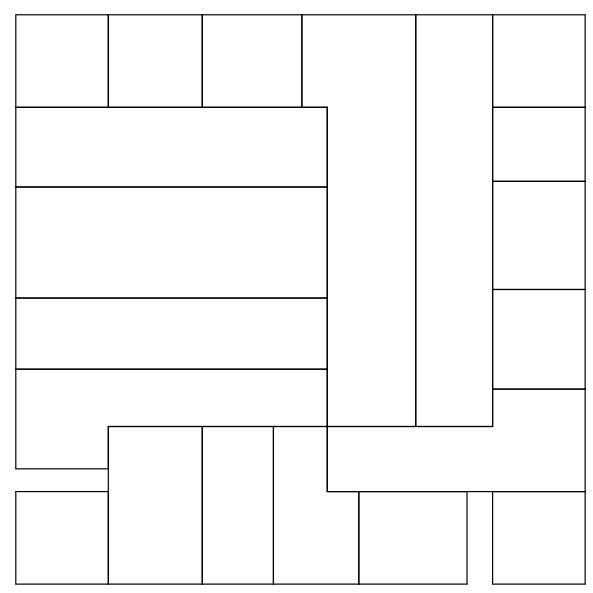

05

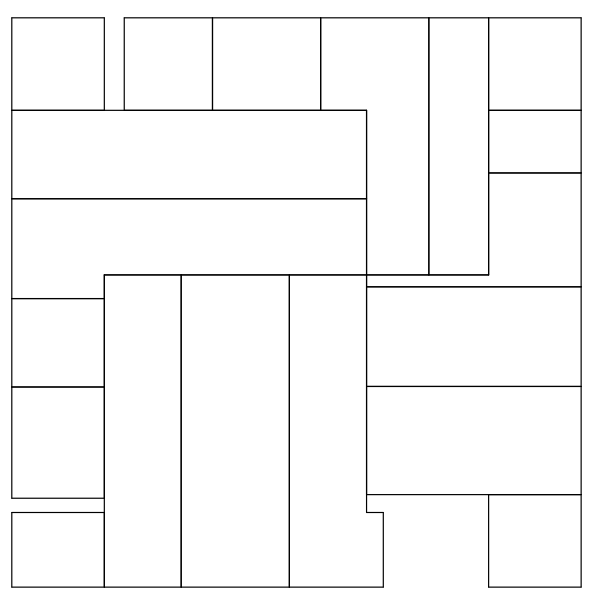

09

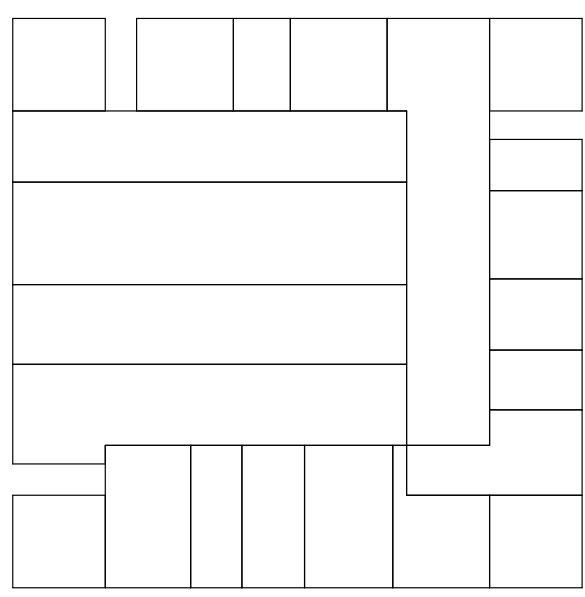

02

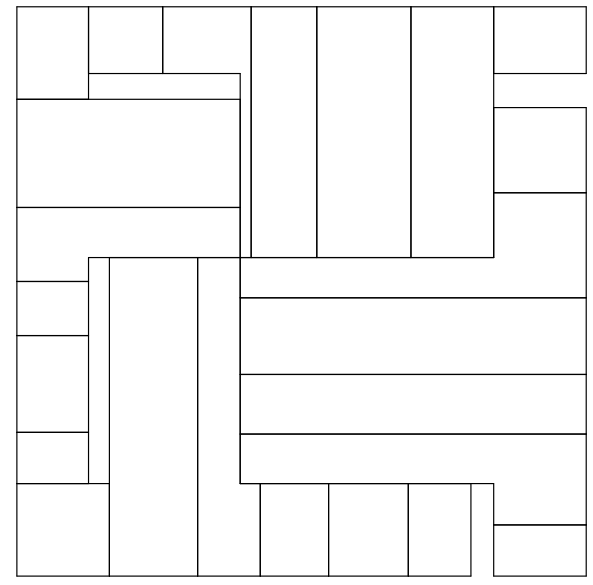

06

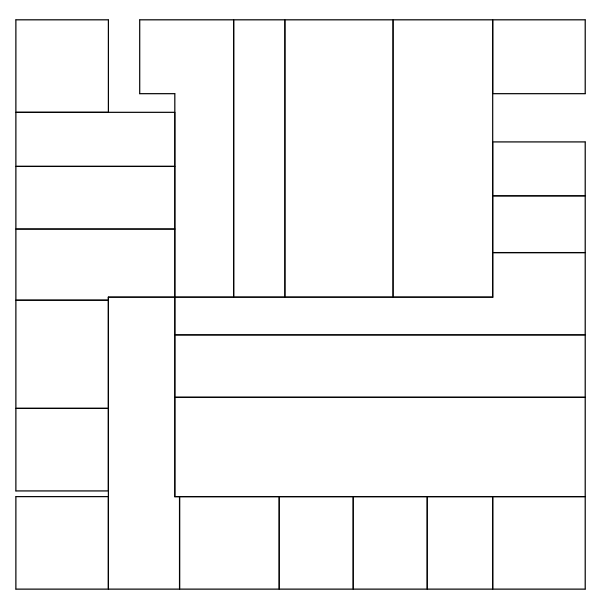

10

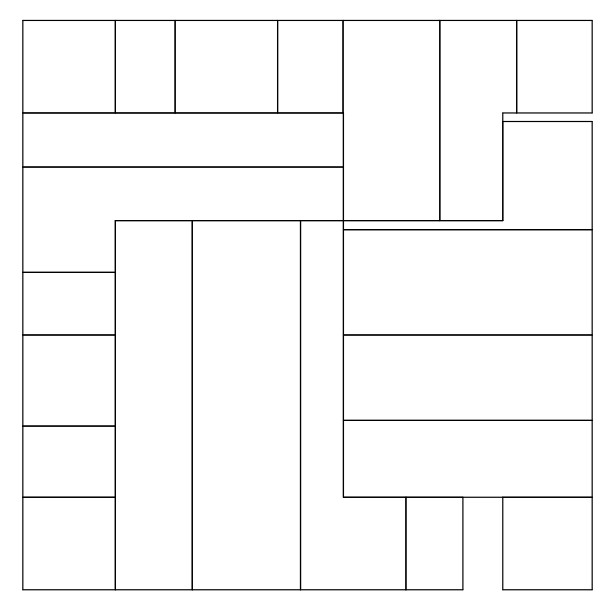

03

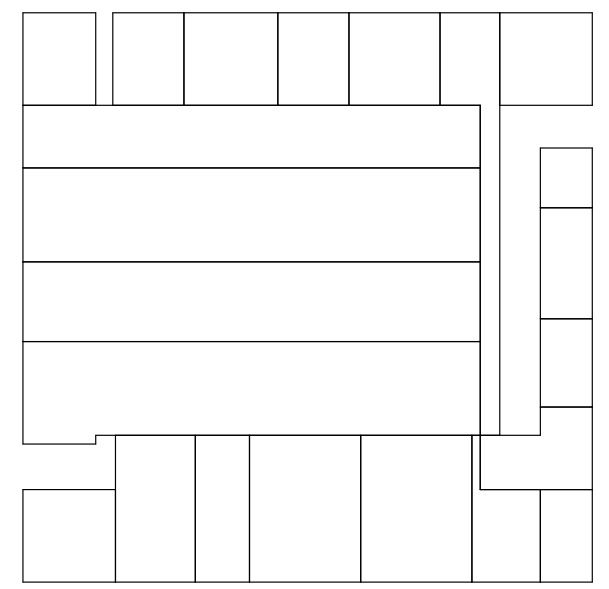

07

\begin{tabular}{|l|r|}
\hline Base Surface Size & $200 \times 200$ metres \\
\hline Minimum Building Widths & $17.5 \mathrm{~m}$ \\
\hline Maximum Building Widths & $32.5 \mathrm{~m}$ \\
\hline
\end{tabular}

Figure 4.12: Compilation of outputs - Increased minimum / decreased maximum width 


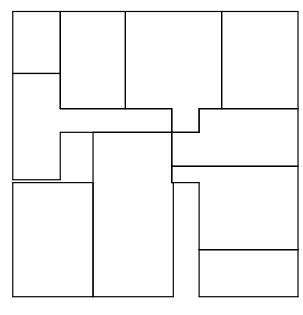

Seed 00

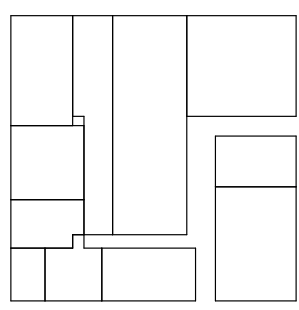

04

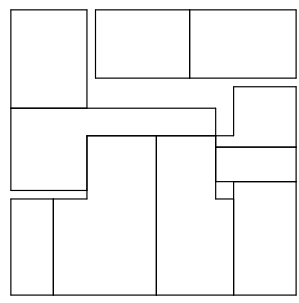

08

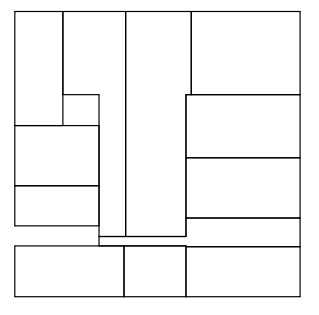

01

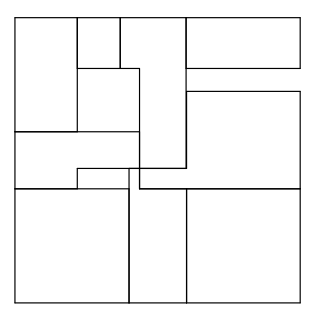

05

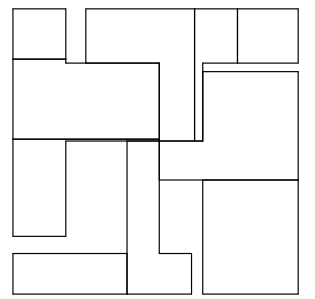

09

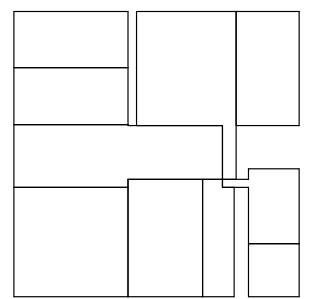

02

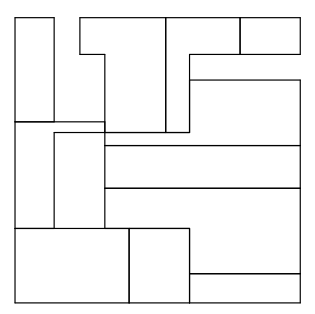

06

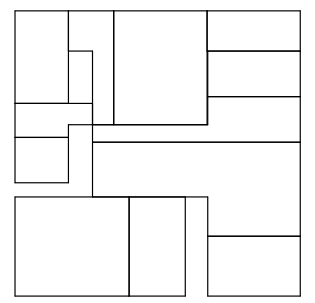

10

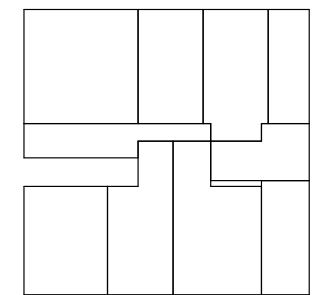

03

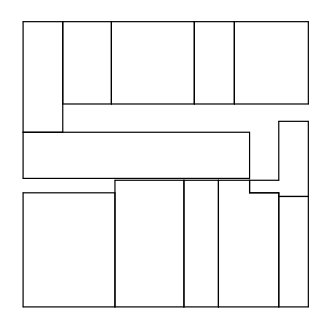

07

\begin{tabular}{|l|r|}
\hline Base Surface Size & $100 \times 100$ metres \\
\hline Minimum Building Widths & $10.0 \mathrm{~m}$ \\
\hline Maximum Building Widths & $40.0 \mathrm{~m}$ \\
\hline
\end{tabular}

Figure 4.13: Compilation of outputs - Smaller base surface

CHAPTER FOUR

A CITY IS NOT A TREE 


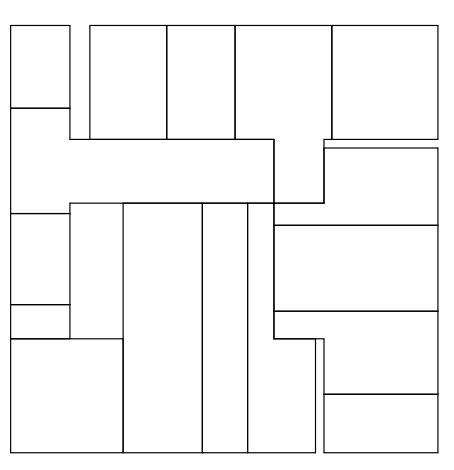

Seed 00

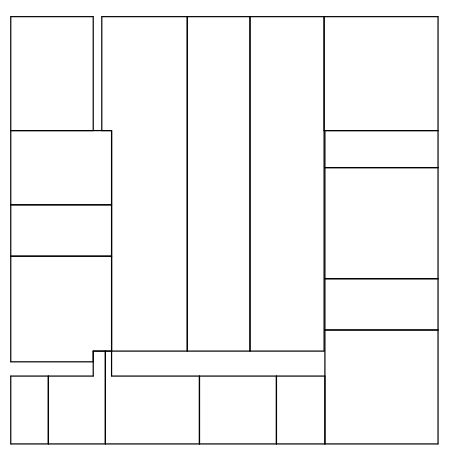

04

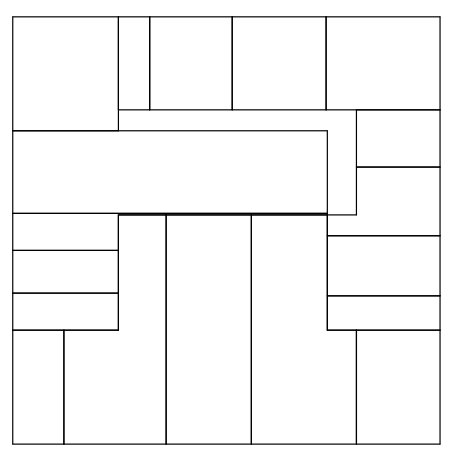

08

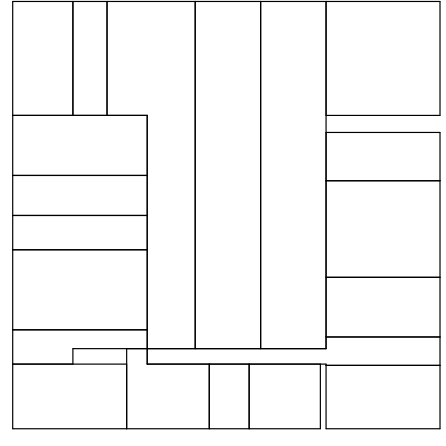

01

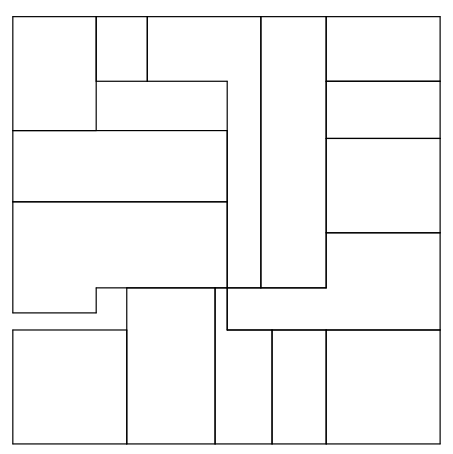

05

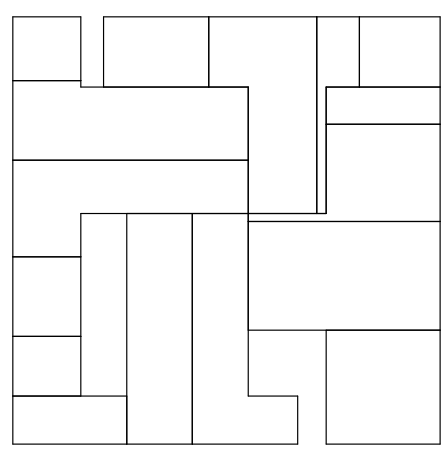

09

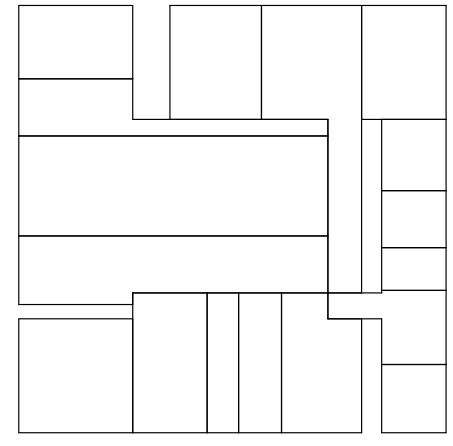

02

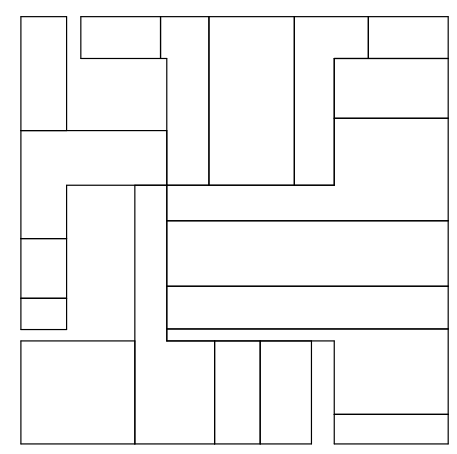

06

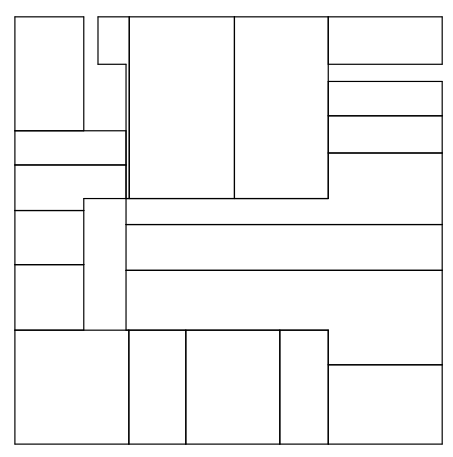

10

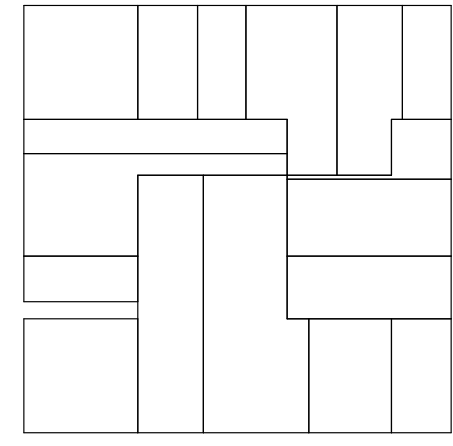

03

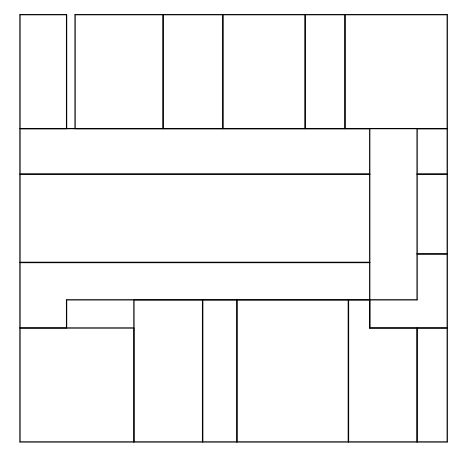

07

\begin{tabular}{|l|r|}
\hline Base Surface Size & $150 \times 150$ metres \\
\hline Minimum Building Widths & $10.0 \mathrm{~m}$ \\
\hline Maximum Building Widths & $40.0 \mathrm{~m}$ \\
\hline
\end{tabular}

Figure 4.14: Compilation of outputs - Small base surface 


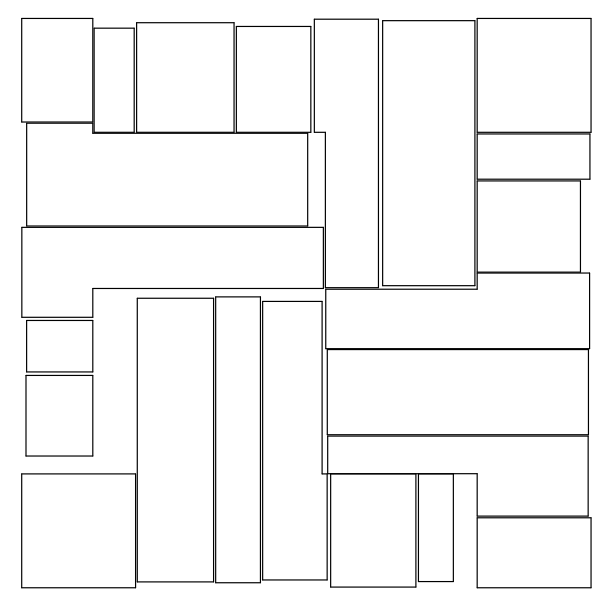

Seed 00

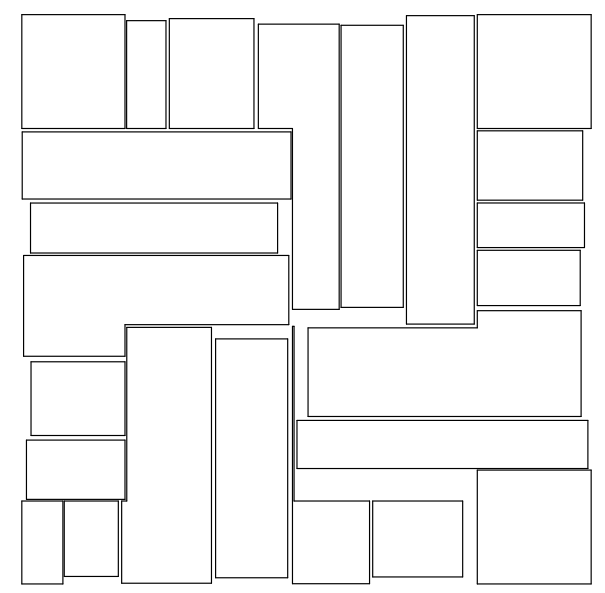

04

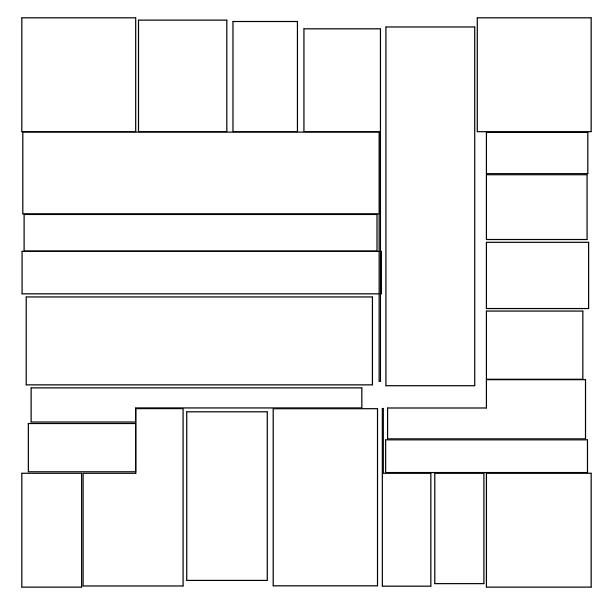

08

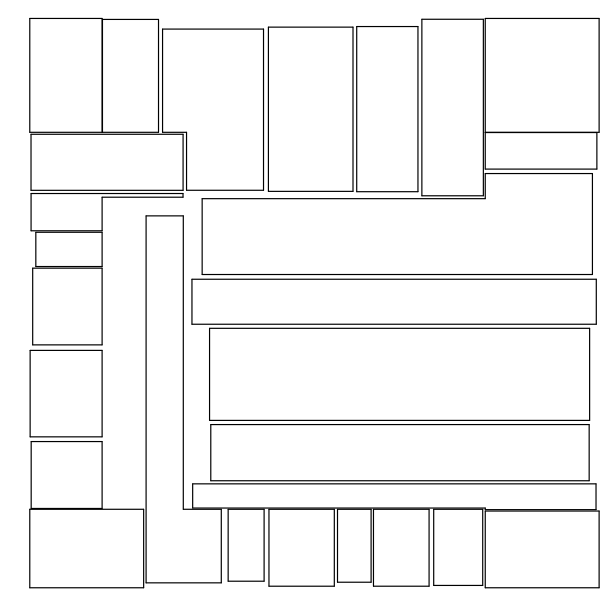

01

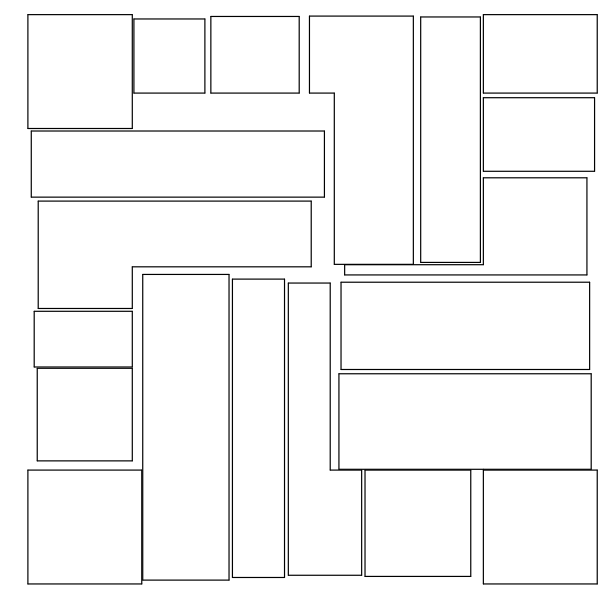

05

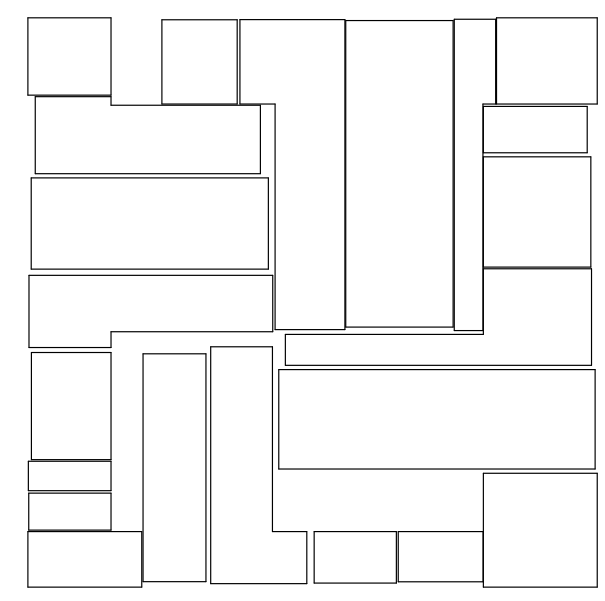

09

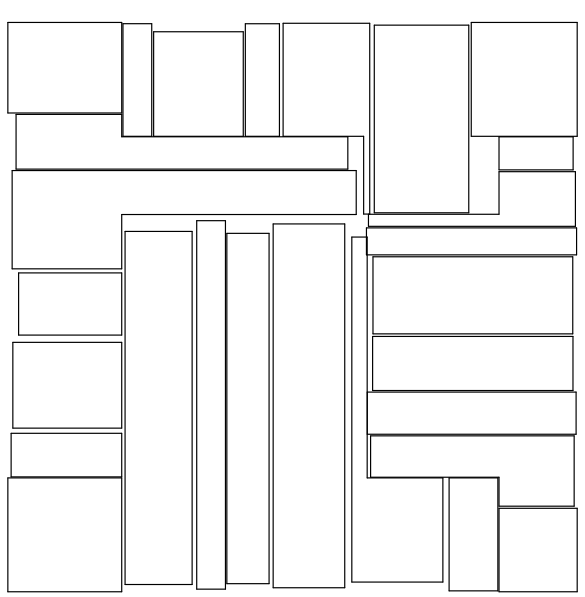

02

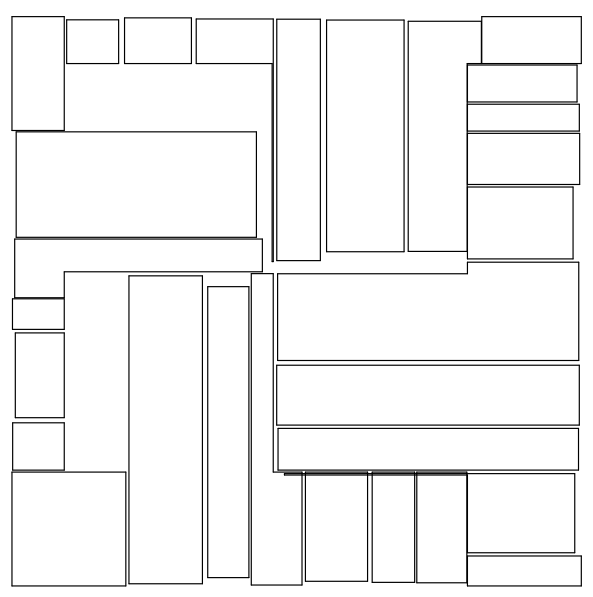

06

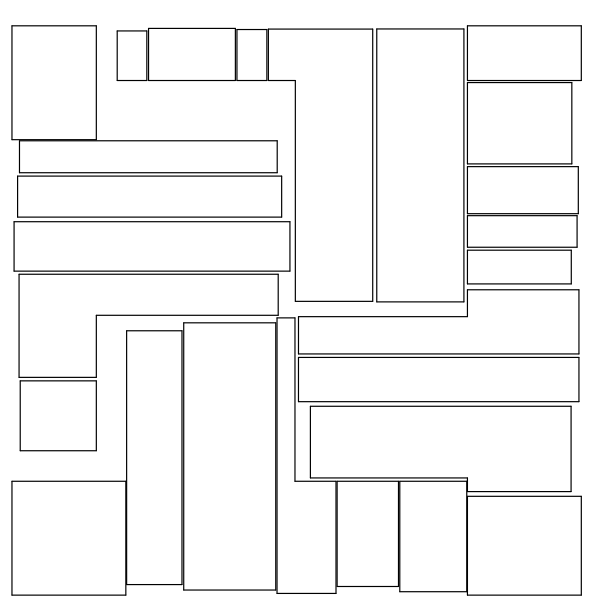

10

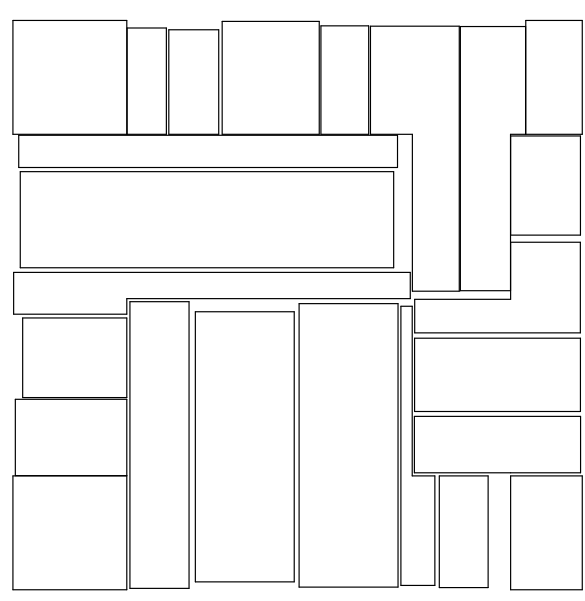

03

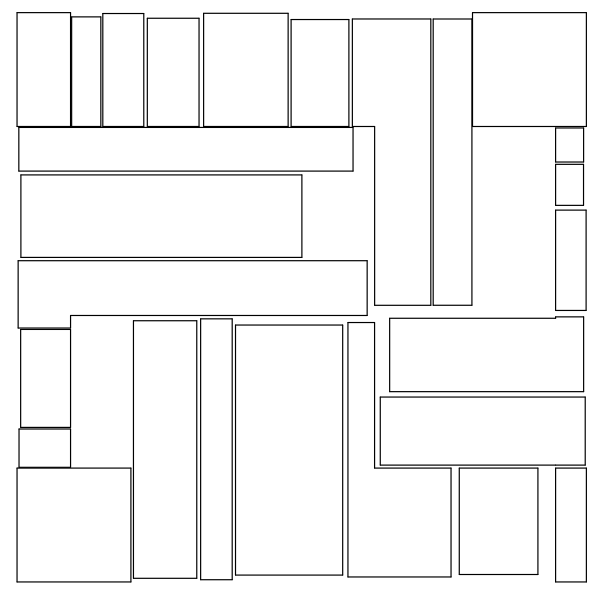

07

\begin{tabular}{|l|r|}
\hline Base Surface Size & $200 \times 200$ metres \\
\hline Building Widths & $10.0 \mathrm{~m}-40.0 \mathrm{~m}$ \\
\hline Length / Width Scaling Range & $0.90-1.00$ \\
\hline
\end{tabular}

Figure 4.15: Compilation of outputs - Length / Width scaling

CHAPTER FOUR

A CITY IS NOT A TREE 


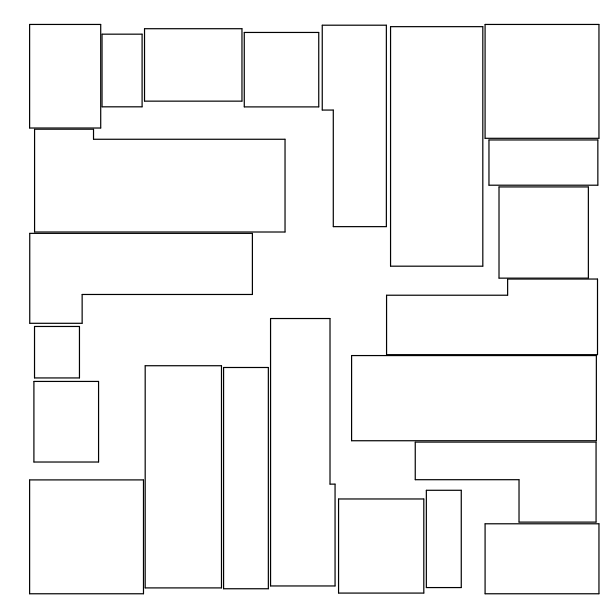

Seed 00

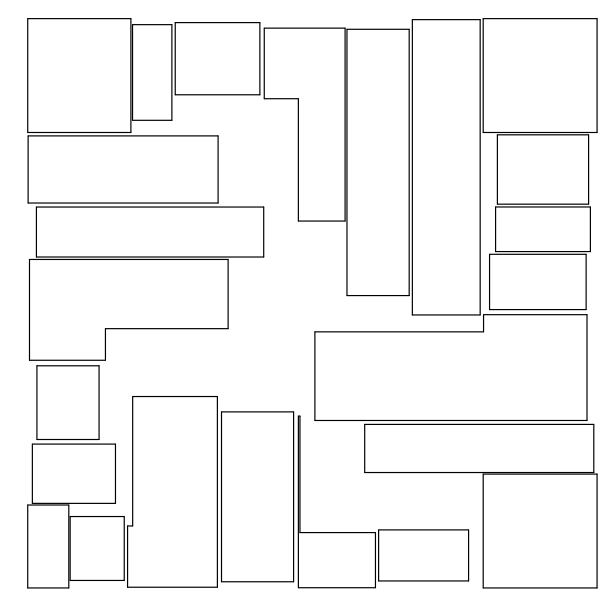

04

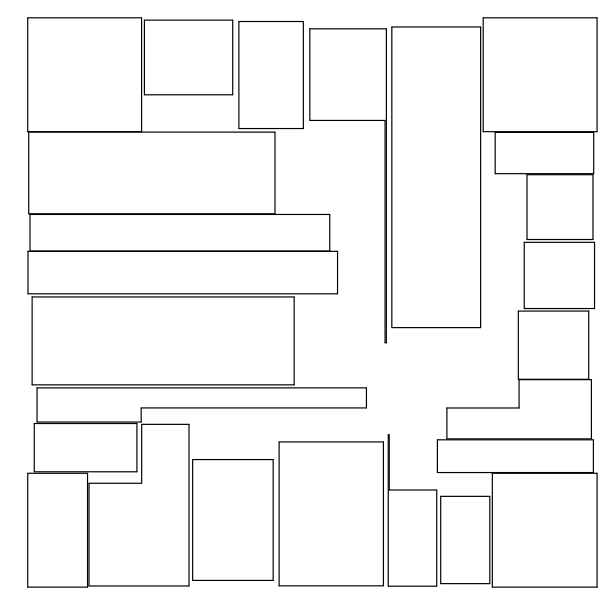

08

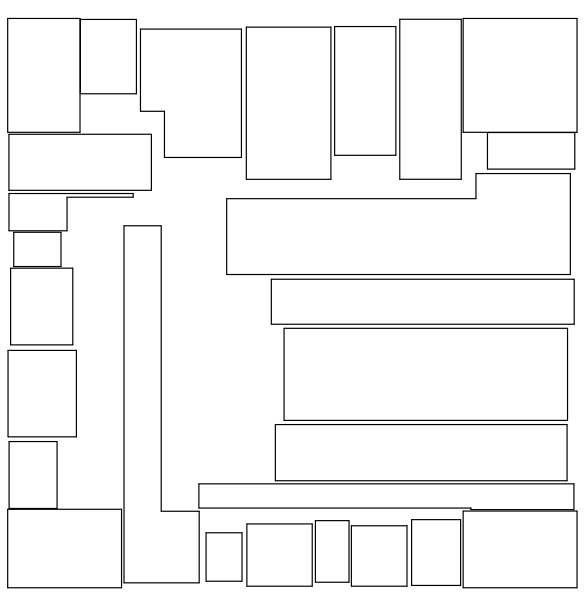

01

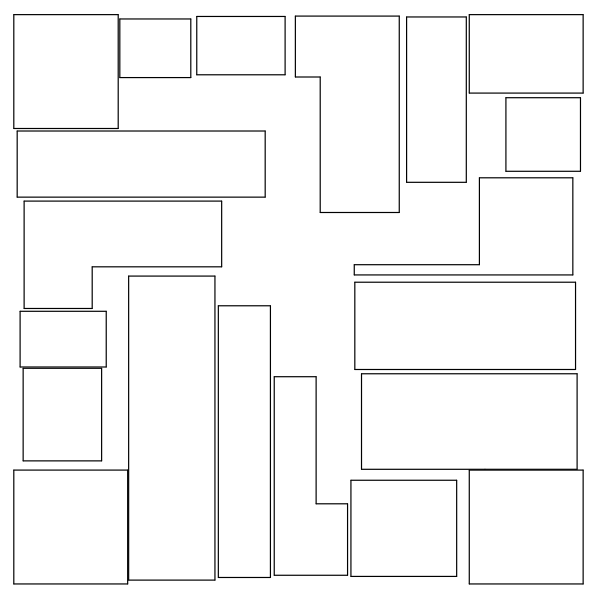

05

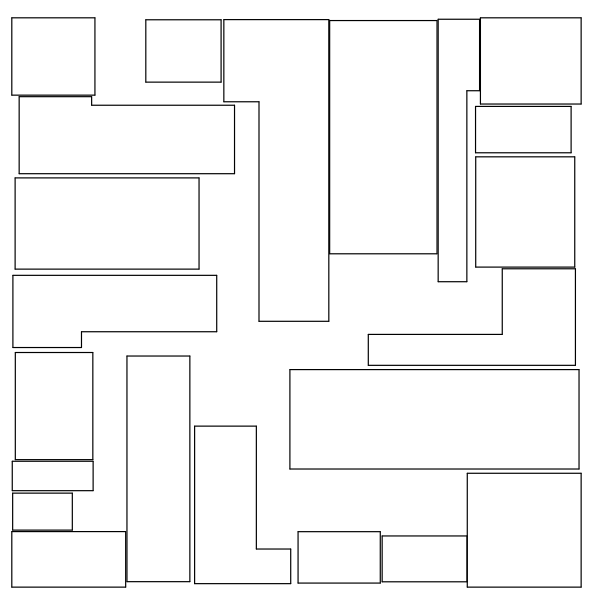

09

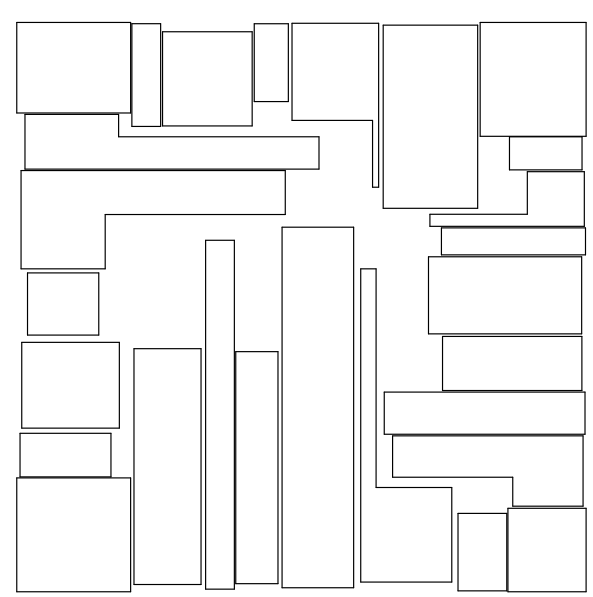

02

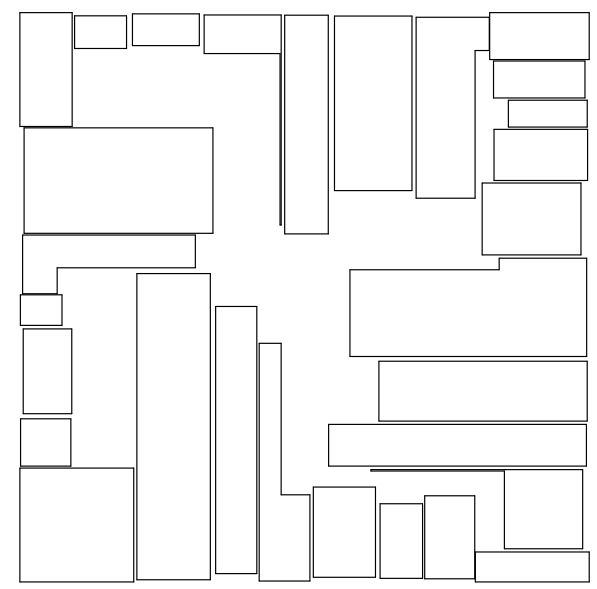

06

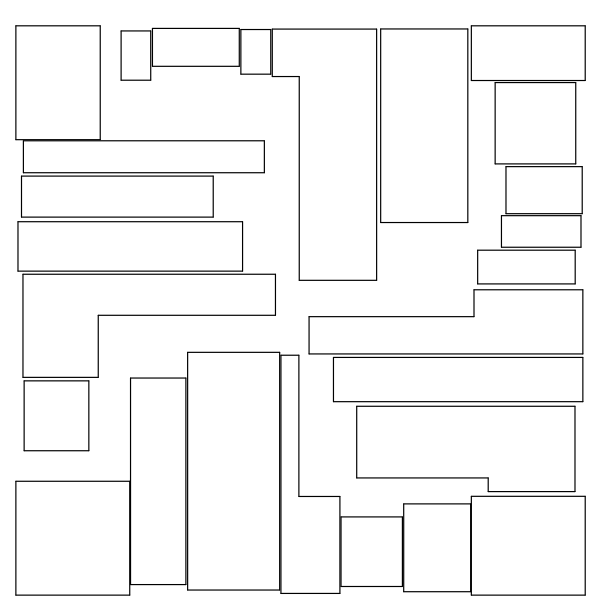

10

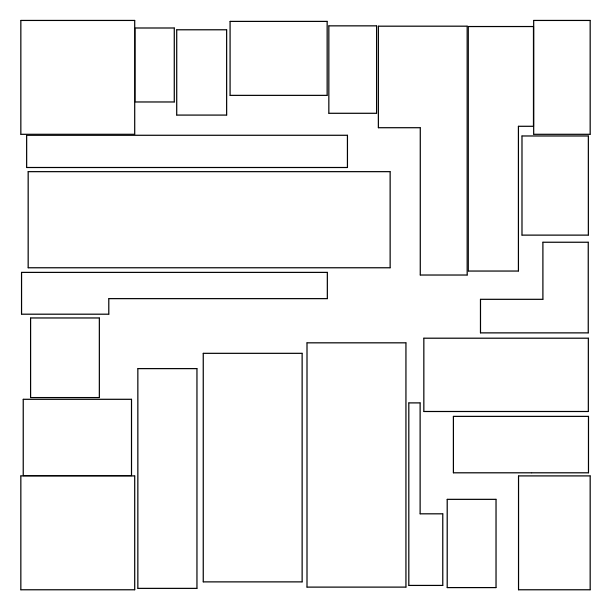

03

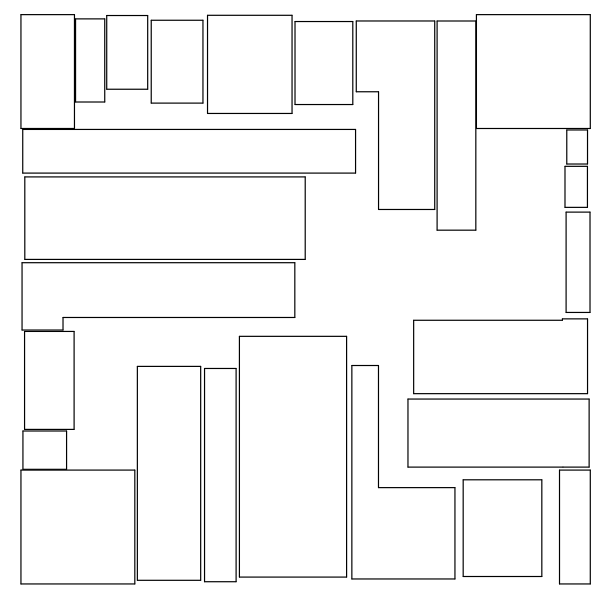

07

\begin{tabular}{|l|r|}
\hline Base Surface Size & $200 \times 200$ metres \\
\hline Building Widths & $10.0 \mathrm{~m}-40.0 \mathrm{~m}$ \\
\hline Length / Width Scaling Range & $0.90-1.00$ \\
\hline Depth Scaling Range & $0.70-1.00$ \\
\hline
\end{tabular}




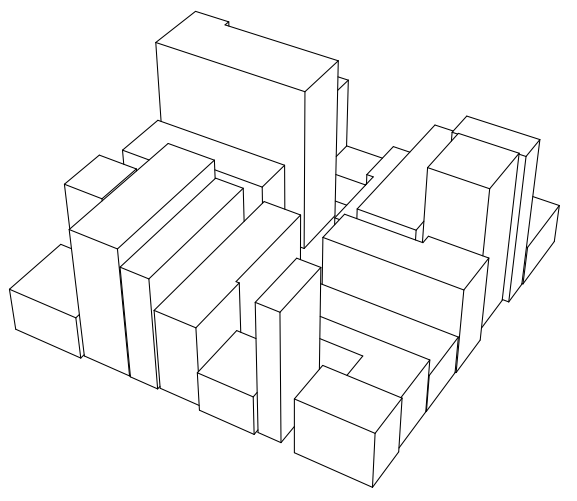

Seed 00

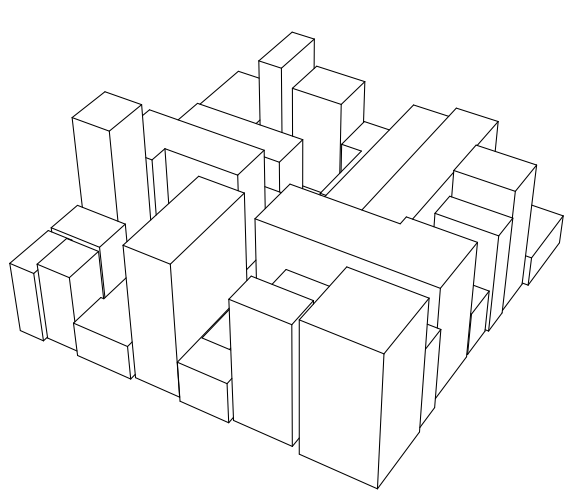

04

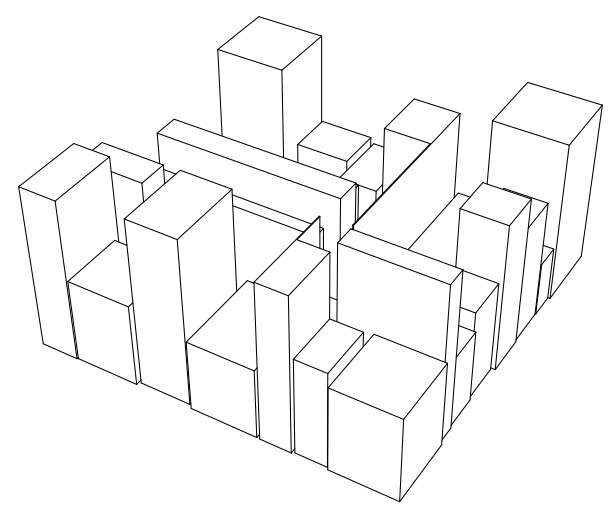

08

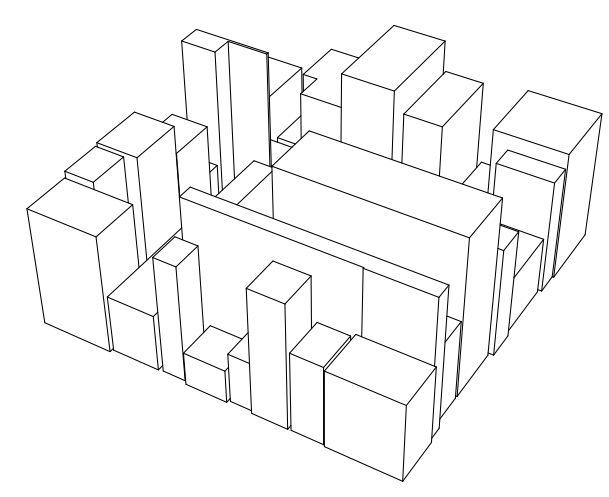

01

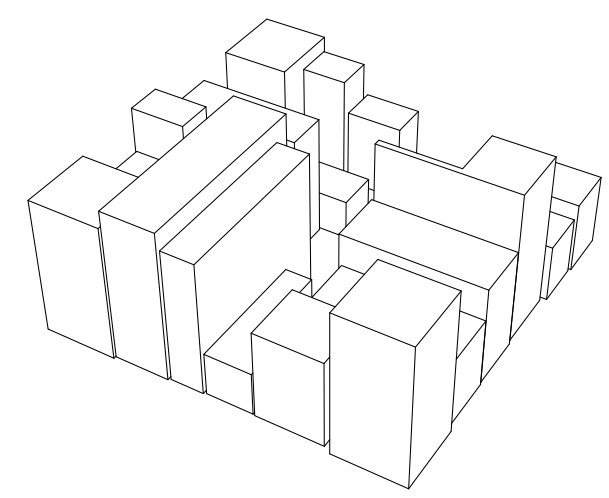

05

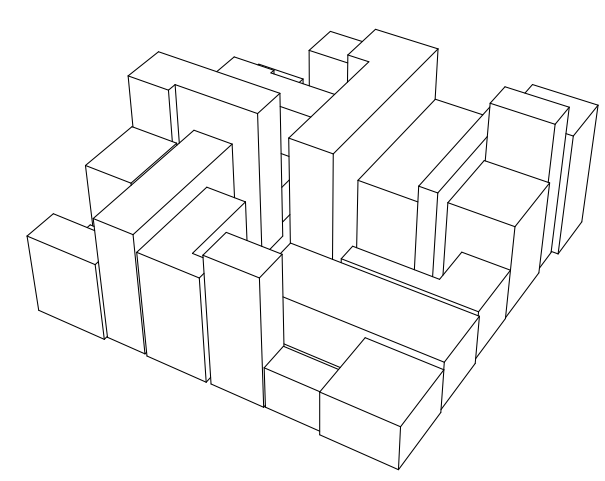

09

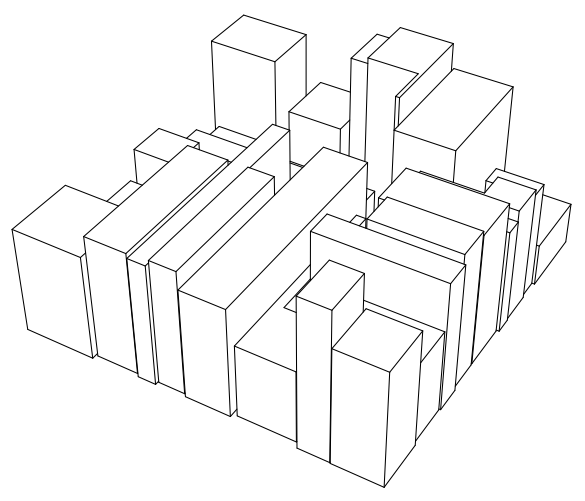

02

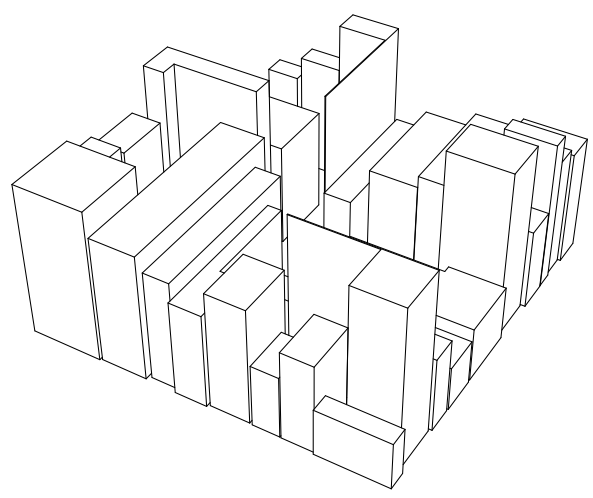

06

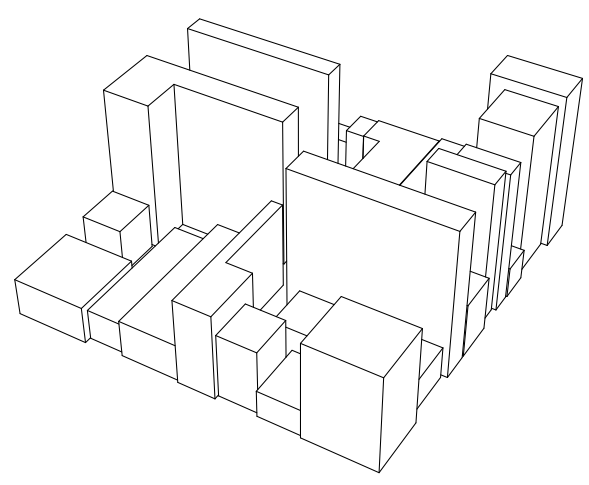

10

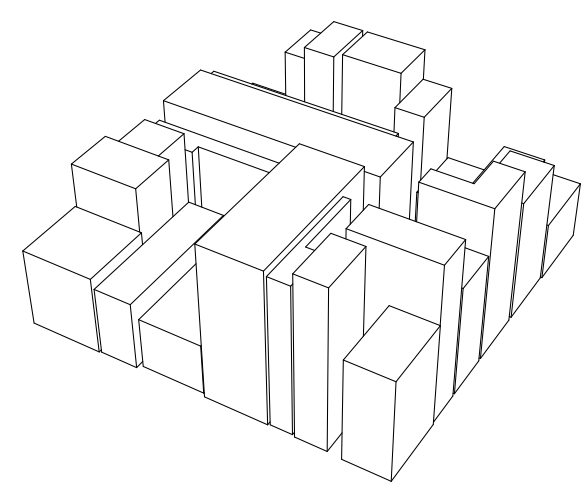

03

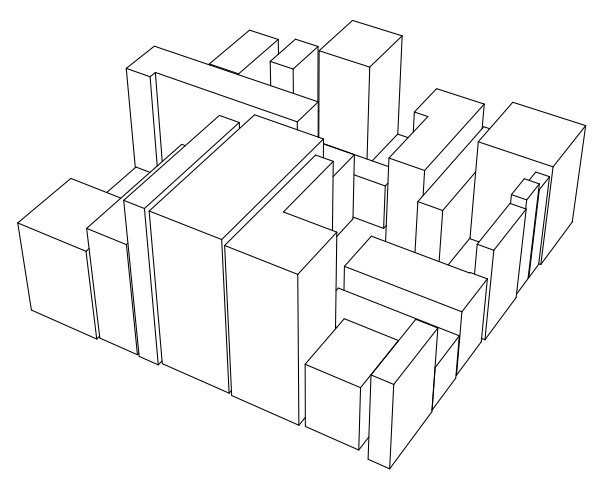

07 15 - 100 metres

Figure 4.17: Compilation of outputs - Extrusion from page 065 outputs

CHAPTER FOUR

A CITY IS NOT A TREE 


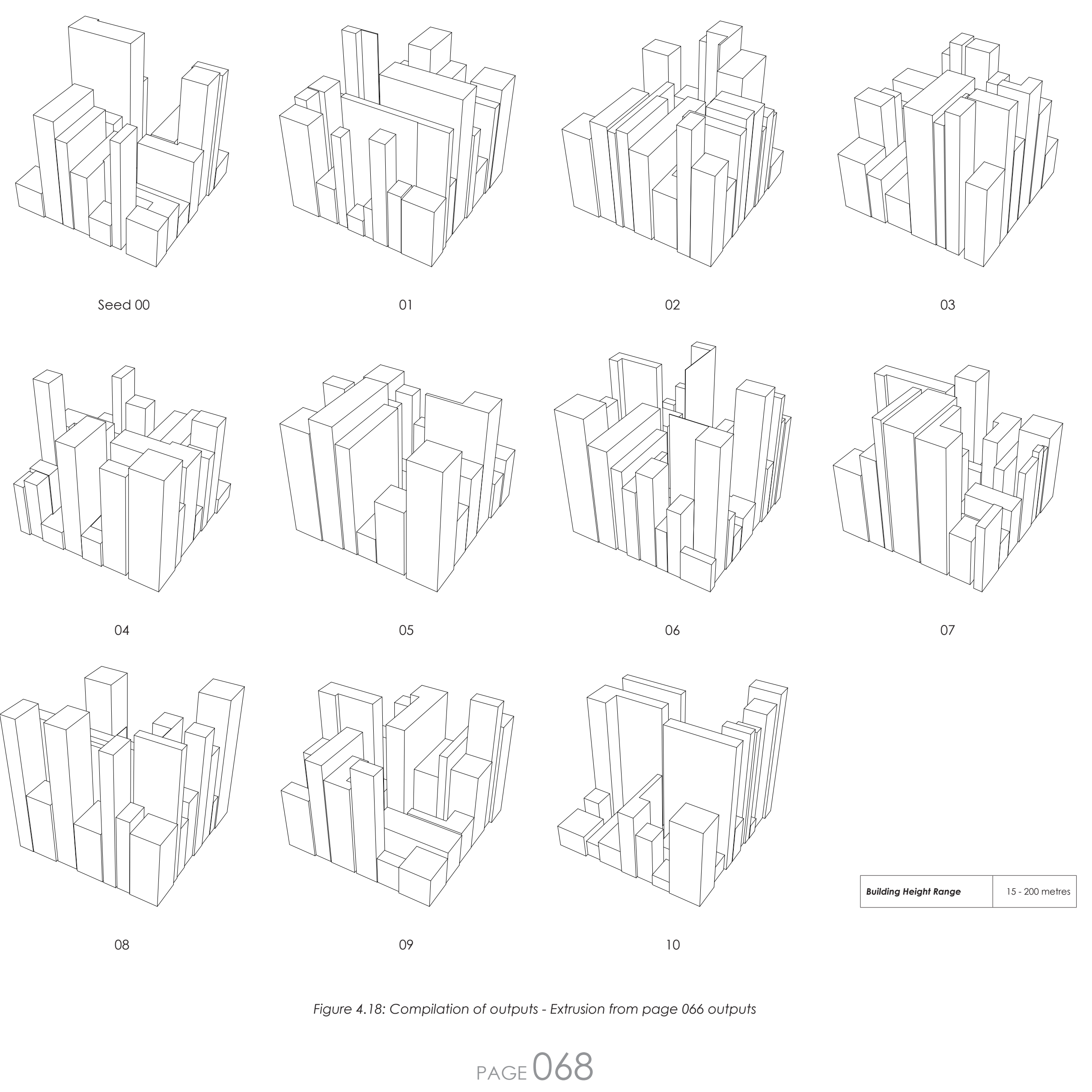




\section{ITERATION FOUR}

- The original surface is scaled, and a point is randomly placed within this scaled area. The original surface is then divided into four sections, using this point. This process ensures that the resultant surfaces are not too small.

- Within the four new surfaces, the same process is undertaken, meaning that the original surface has now been broken into 16 pieces

- Each of the four blocks contains three sections touching the original surface's edge. The external corner block, as well as a dark and light grey block on either side.

- Based on RNG one of the grey sections in each block is joined with the internal segment to create one larger block

- The twelve blocks are divided based on a minimum/maximum width value and RNG to establish building footprints

- The footprints then subject to a user specified maximum width/ depth ratio. If the width/depth ratio exceeds the user specified value exceeds the footprint is scaled to the user defined value

- The footprint, scaled or unscaled, undergoes randomised scaling from its centre 


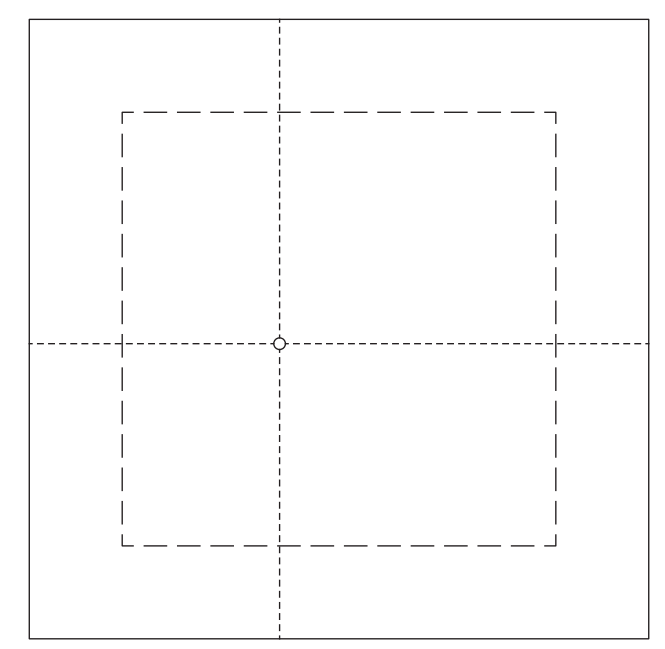

Step 01

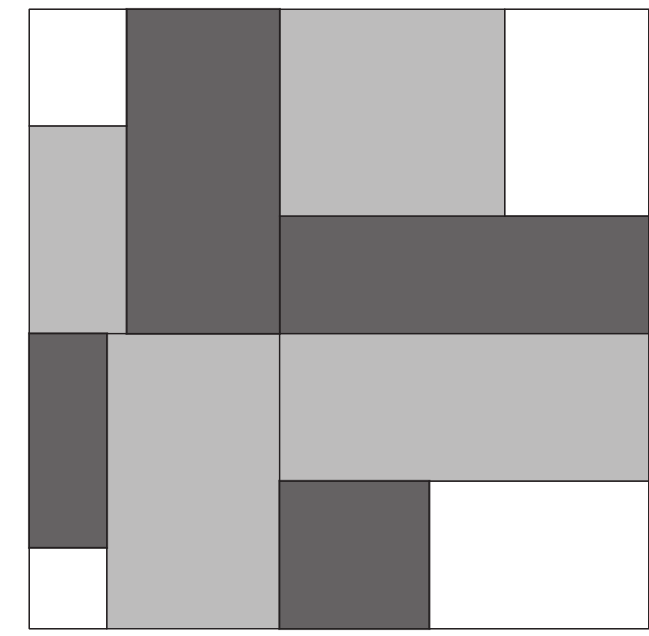

04

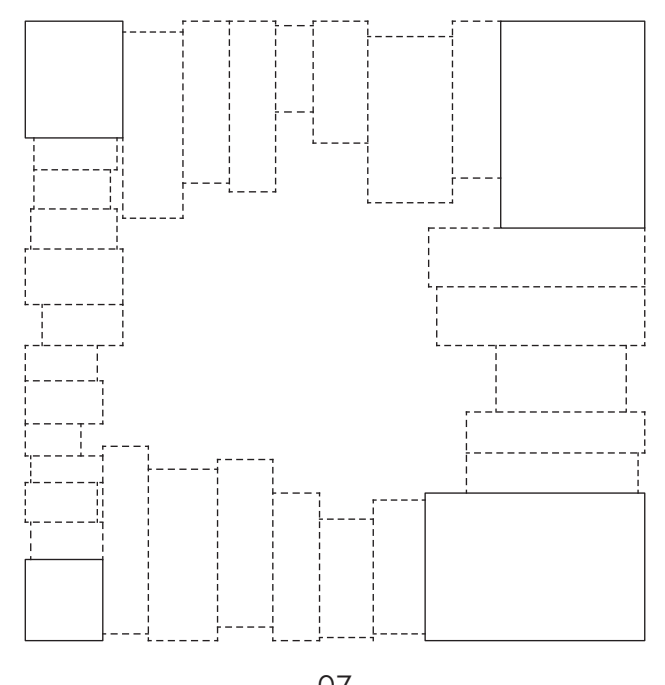

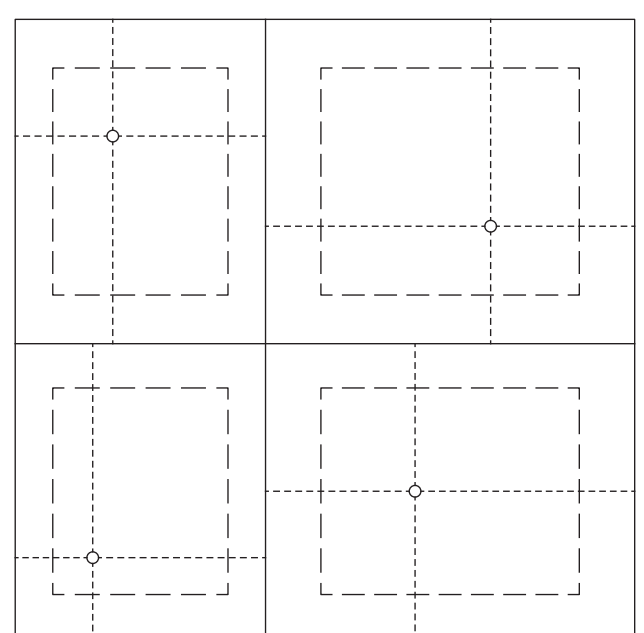

02

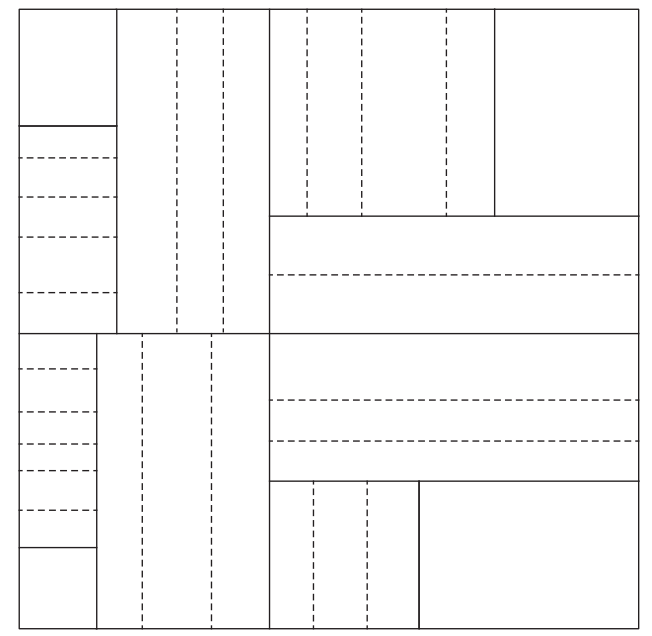

05

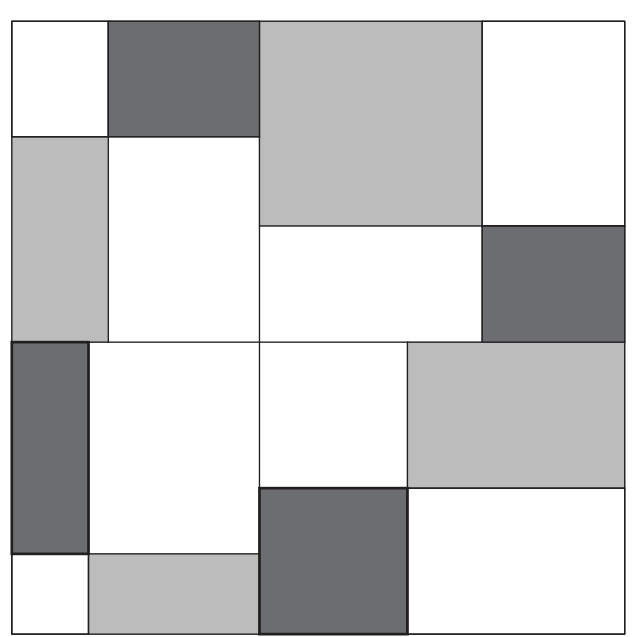

03

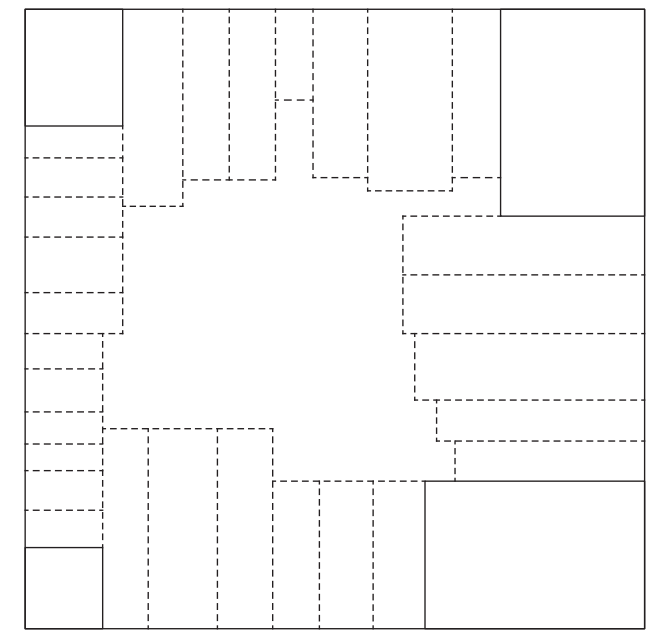

06

Figure 4.19: Construction logic for creating Iteration Four blocks 


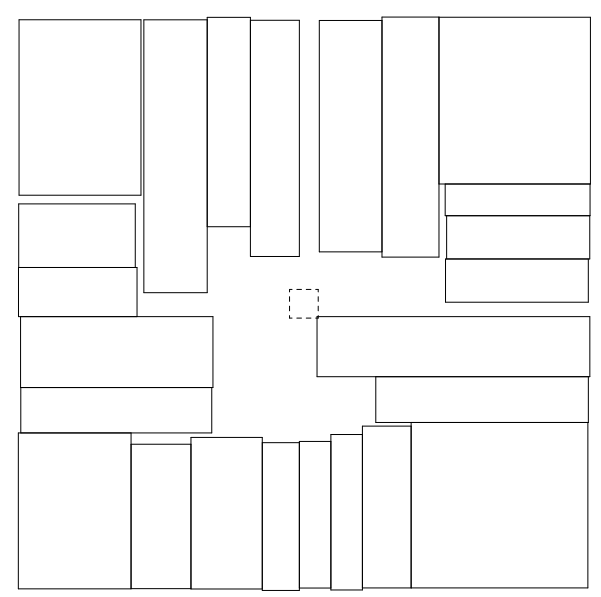

Seed 01: Scaling 5\%

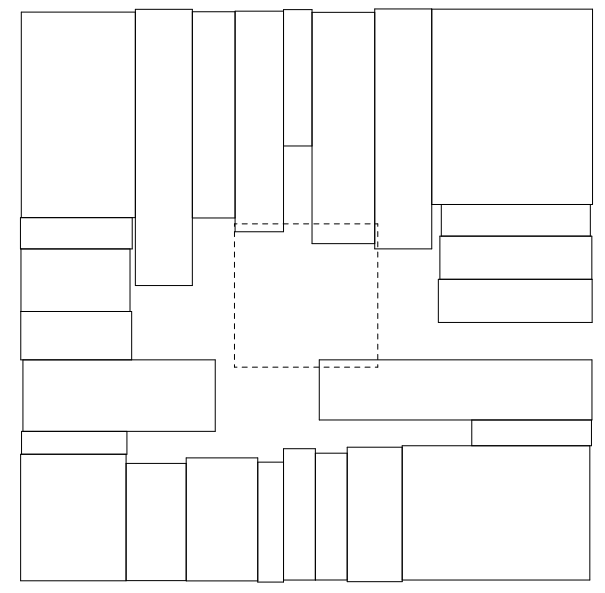

$25 \%$

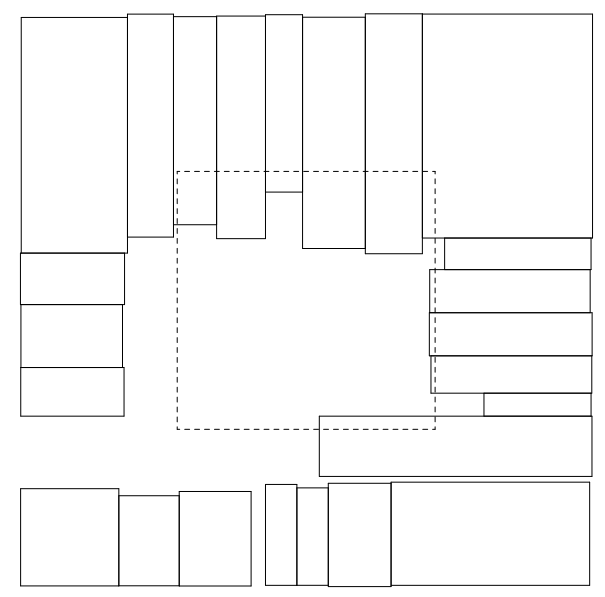

$45 \%$

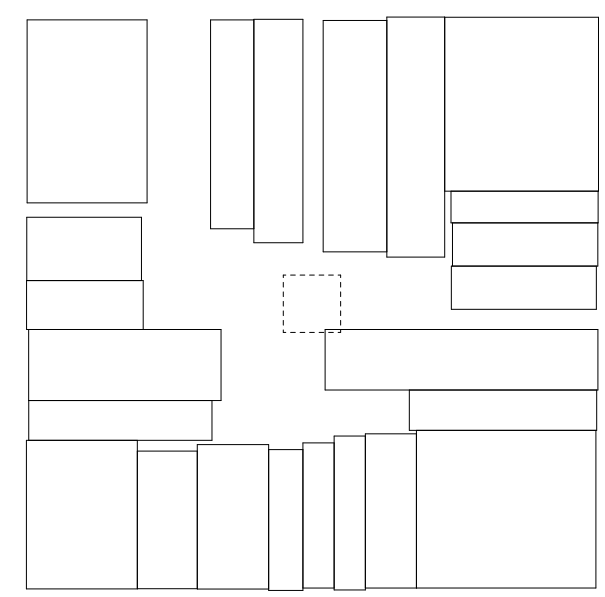

$10 \%$

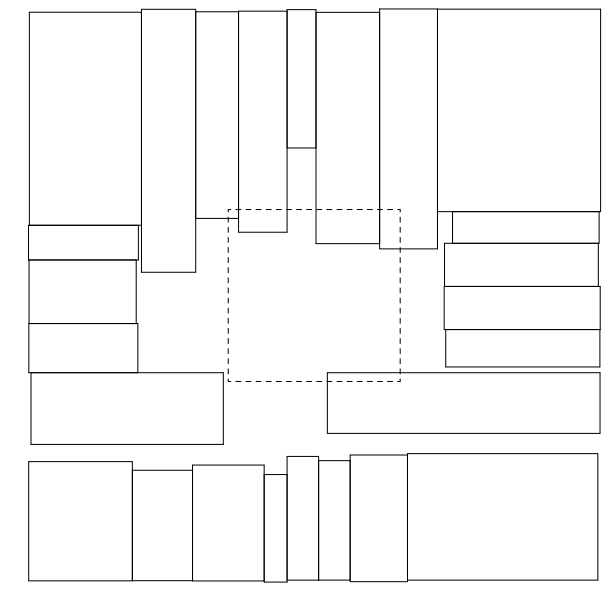

$30 \%$

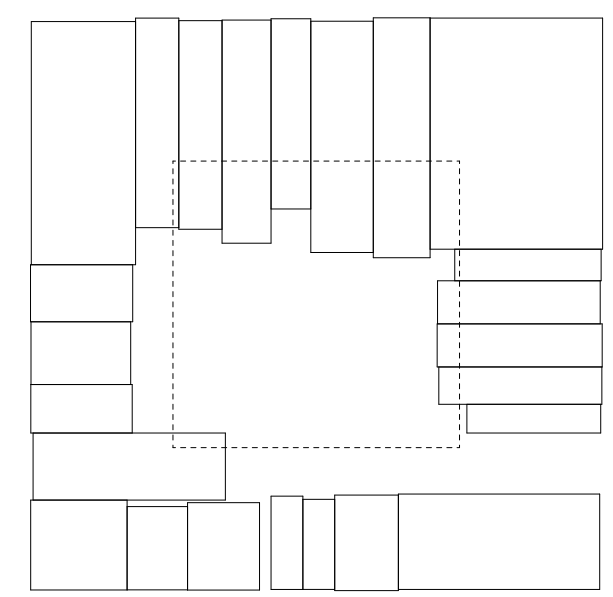

$50 \%$

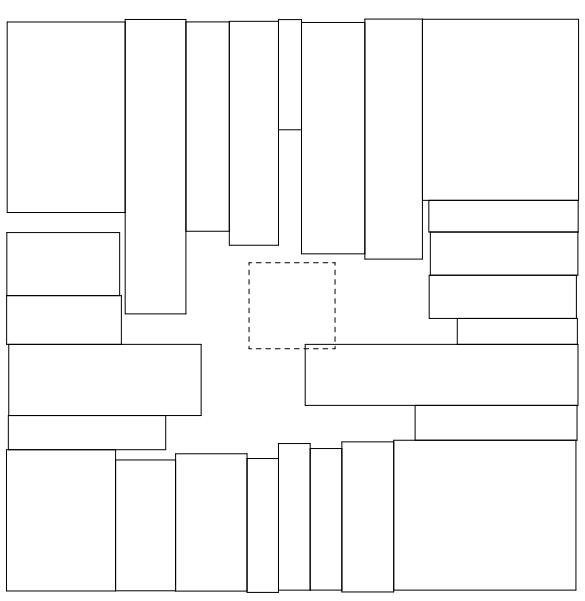

$15 \%$

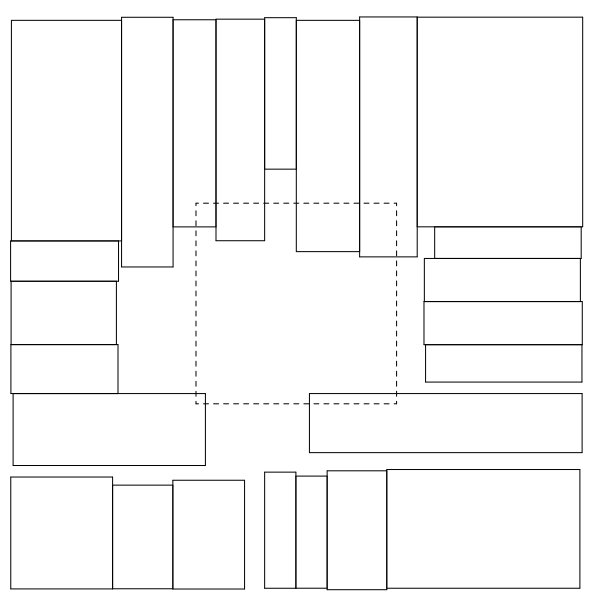

$35 \%$

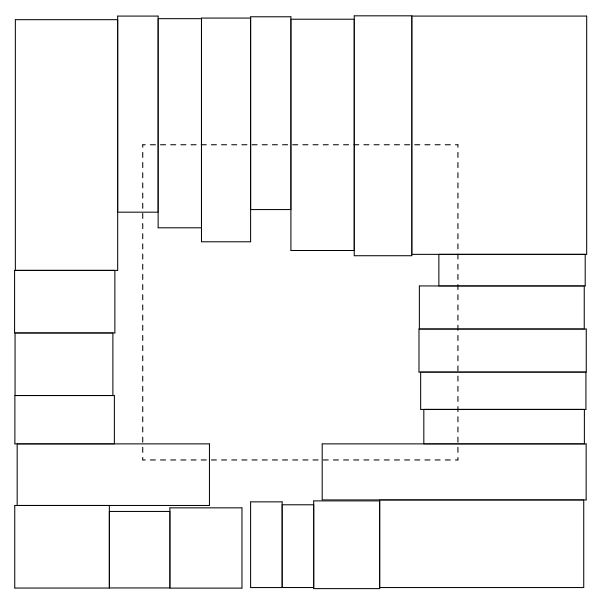

$55 \%$

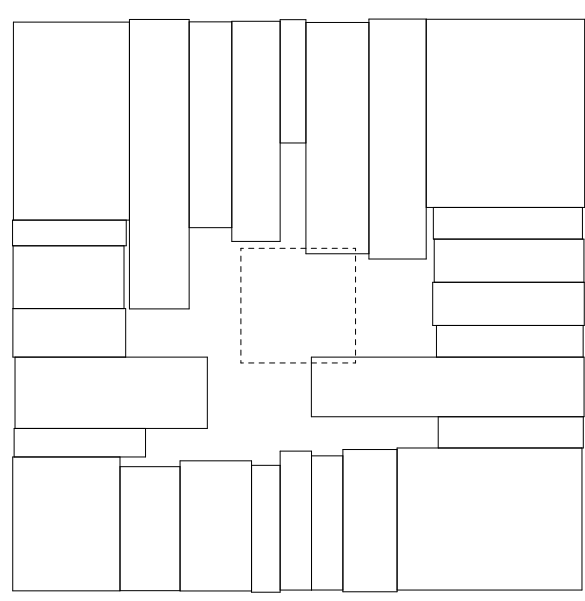

$20 \%$

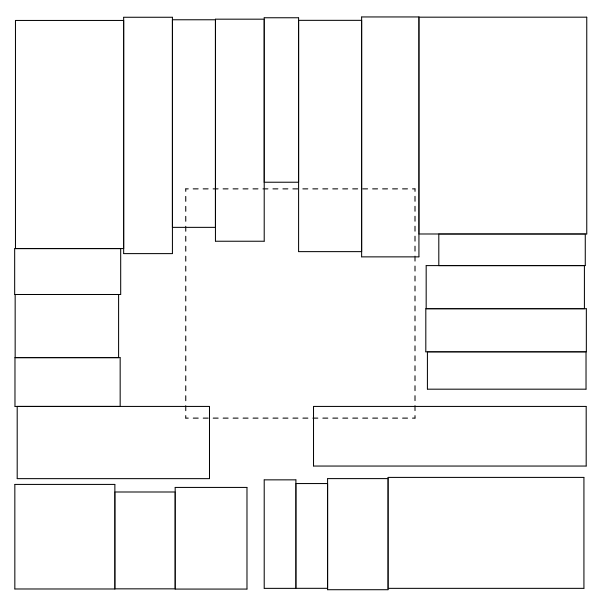

$40 \%$

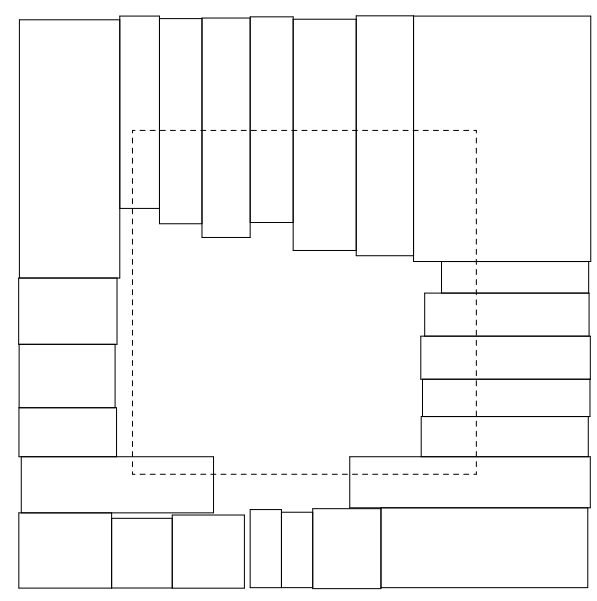

$60 \%$

Figure 4.20: Compilation of outputs - centre scaling comparison

CHAPTER FOUR

A CITY IS NOT A TREE 


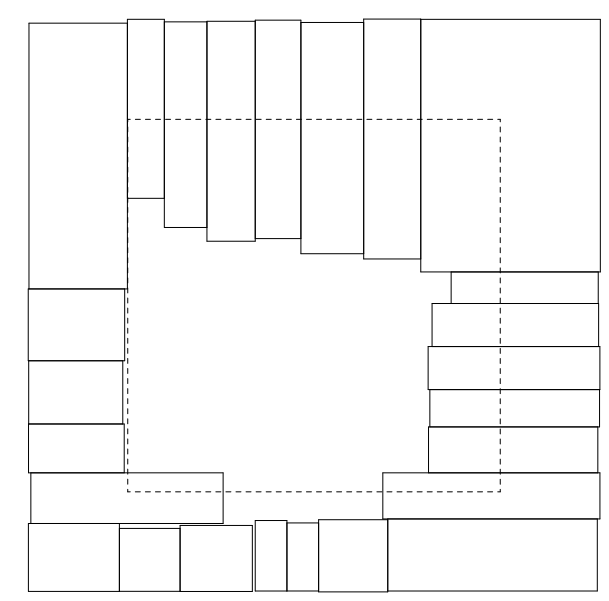

$65 \%$

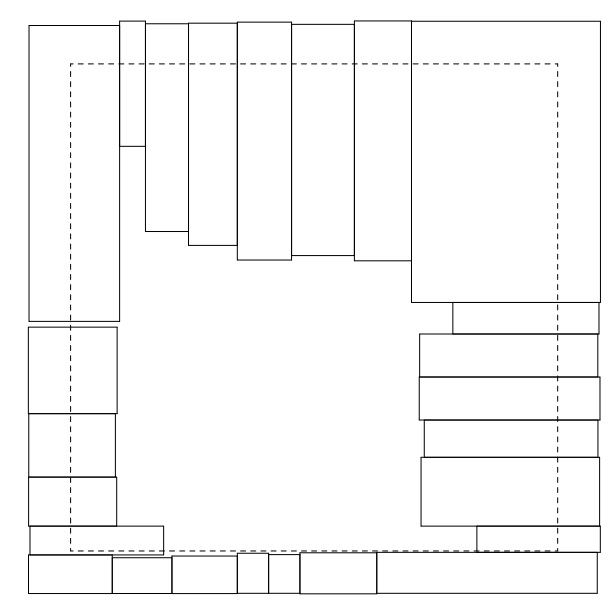

$85 \%$

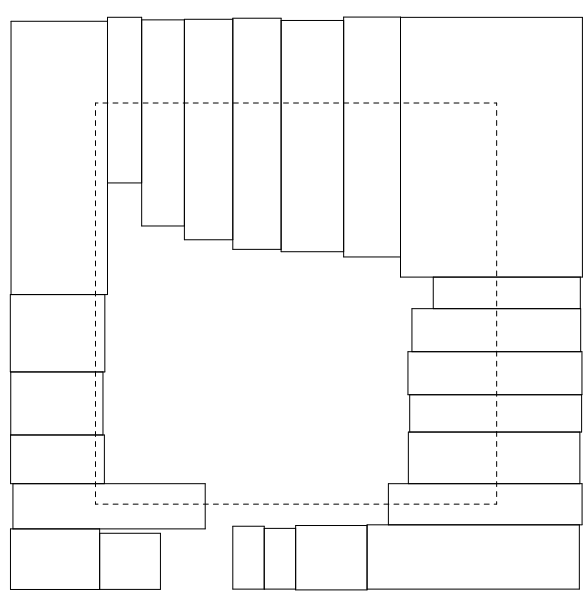

$70 \%$

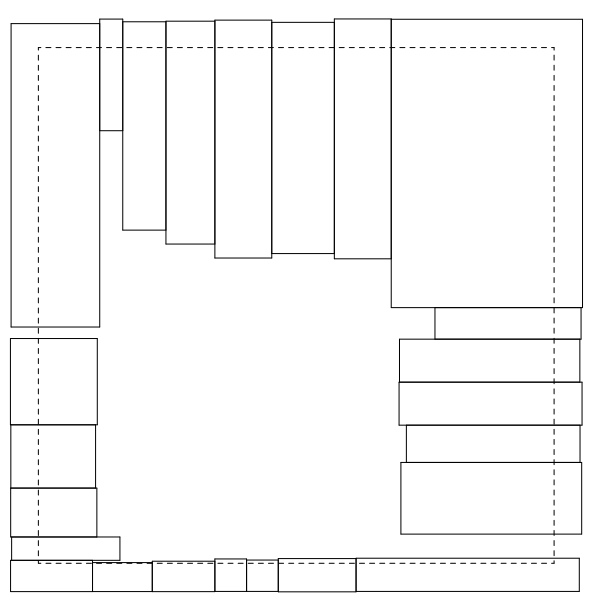

$90 \%$

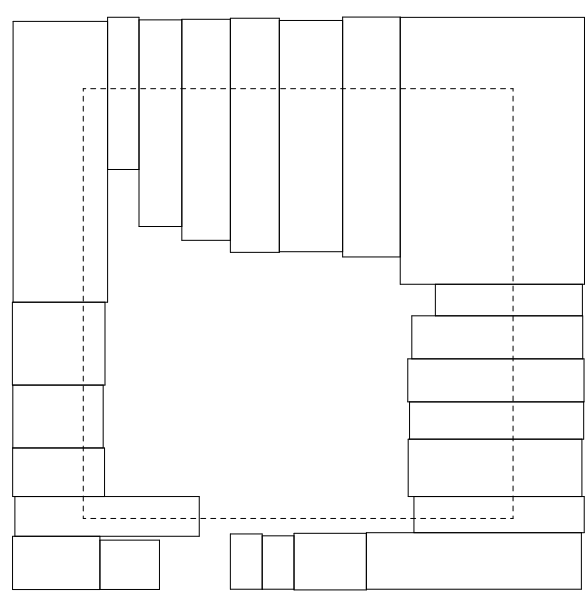

$75 \%$

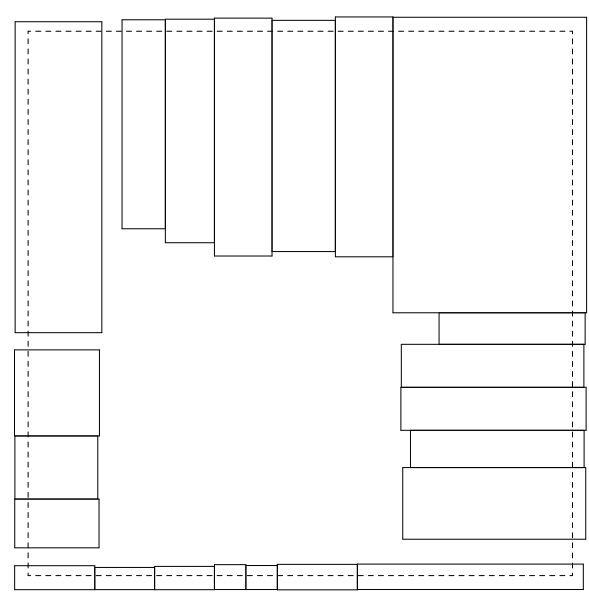

$95 \%$

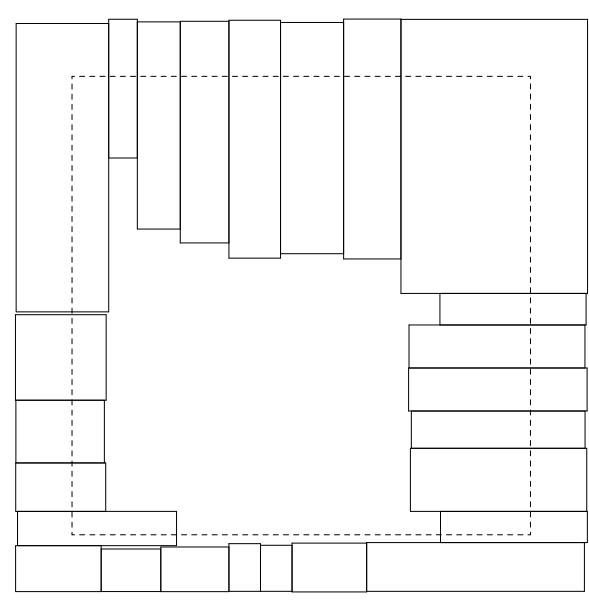

$80 \%$

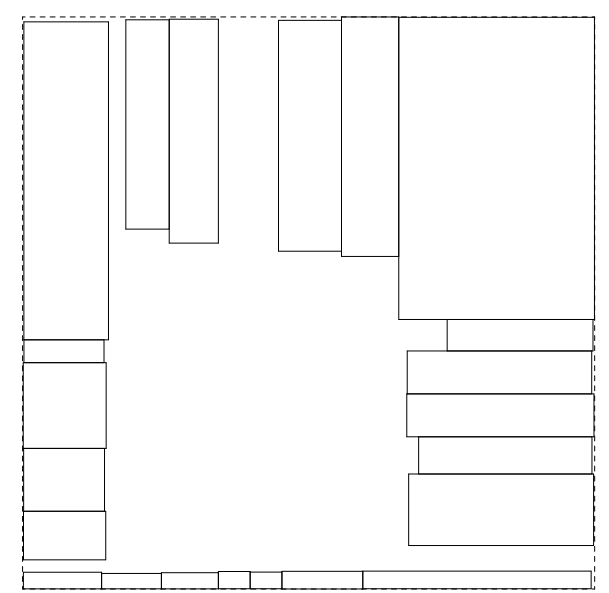

$100 \%$

\begin{tabular}{|l|r|}
\hline Base Surface Size & $125 \times 125$ metres \\
\hline Width: depth Ratio & 5.0 Maximum \\
\hline Minimum Width Range & $7.5 \mathrm{~m}-20.0 \mathrm{~m}$ \\
\hline Maximum Width Range & $20.0 \mathrm{~m}-45.0 \mathrm{~m}$ \\
\hline
\end{tabular}




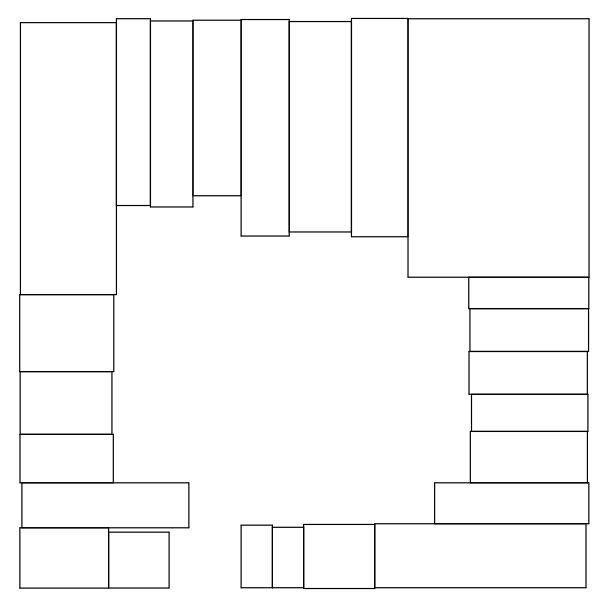

Seed 00

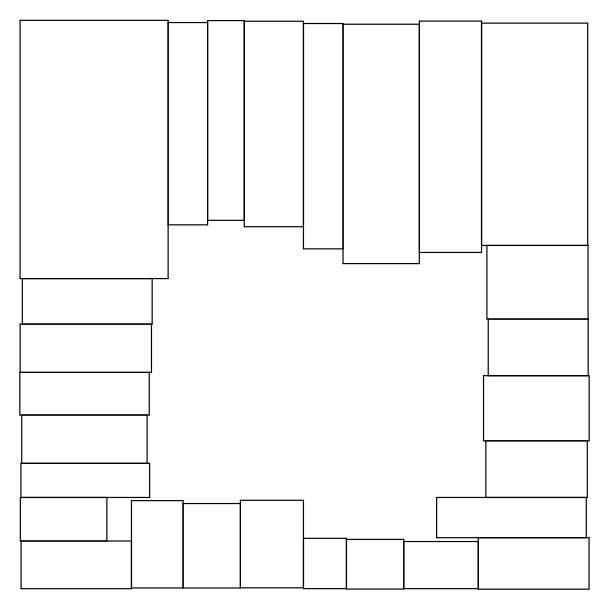

04

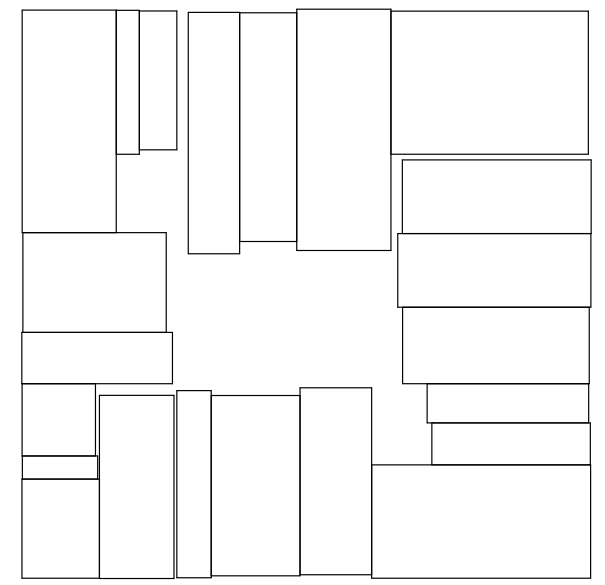

08

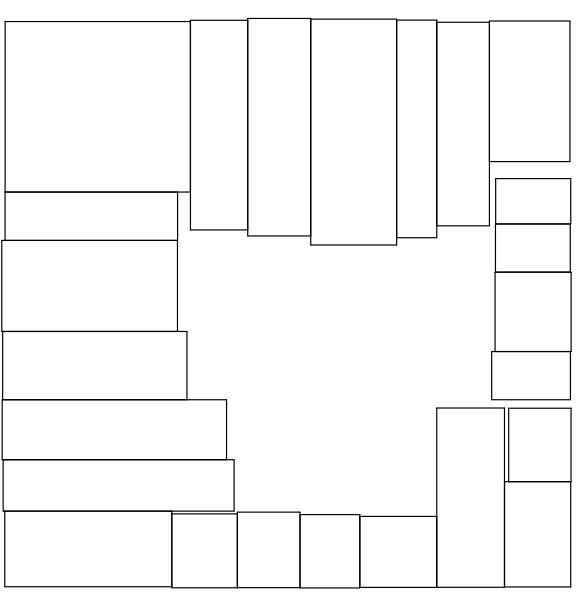

01

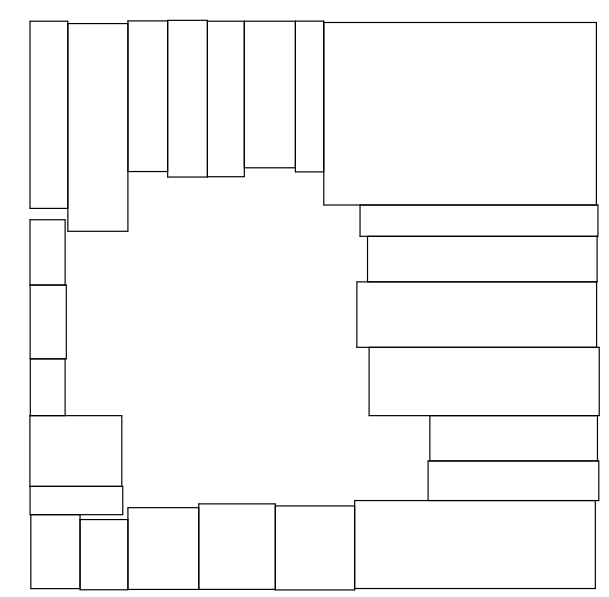

05

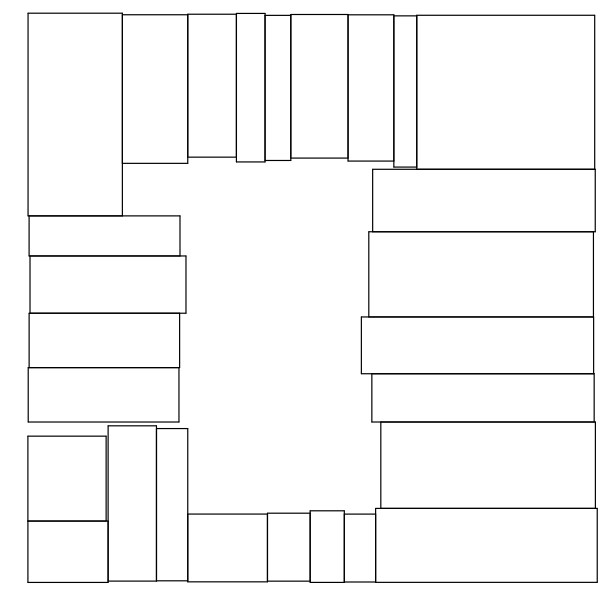

09

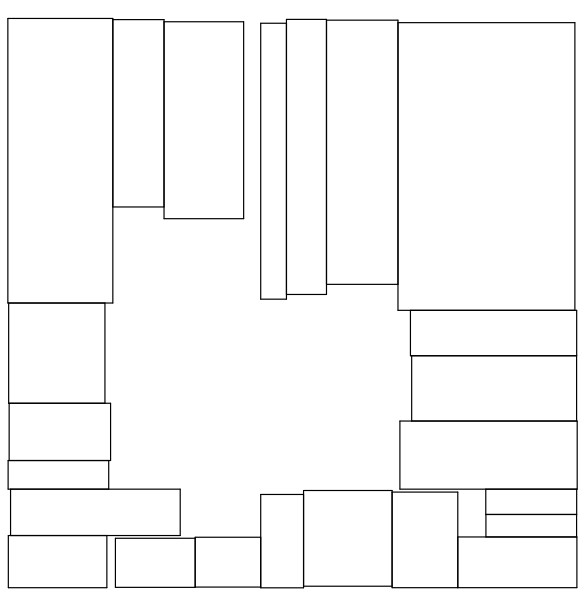

02

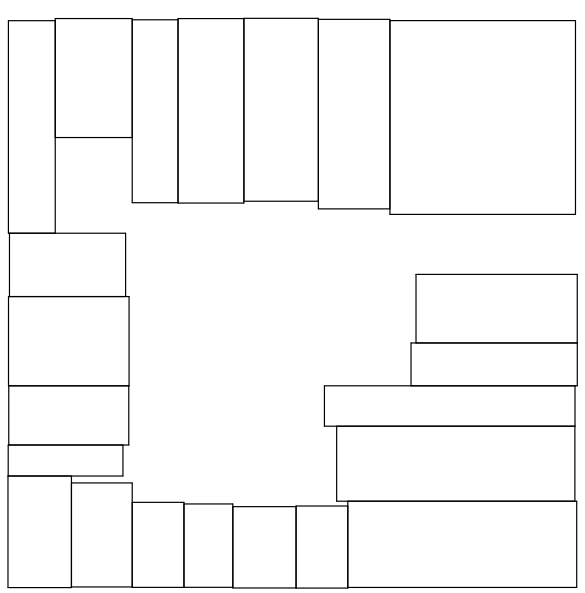

06

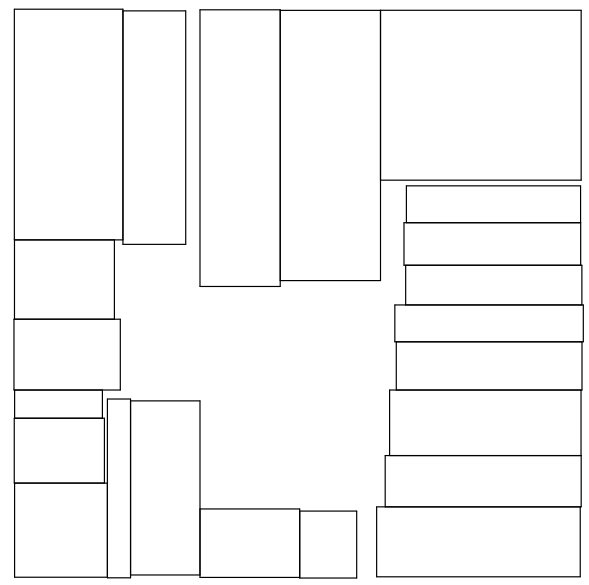

10

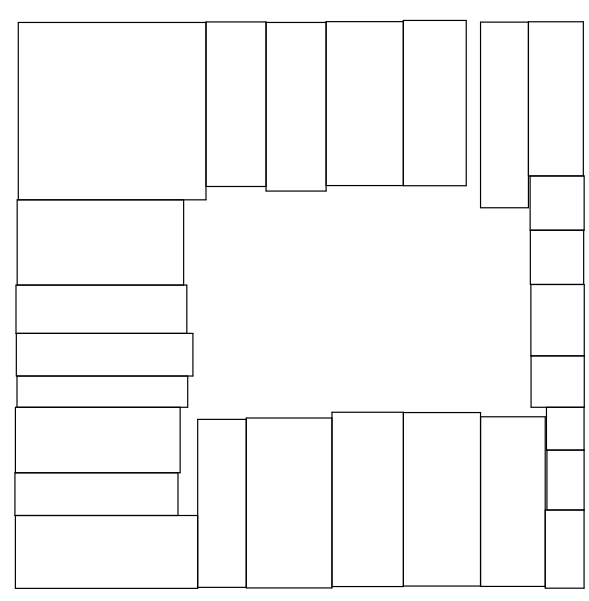

03

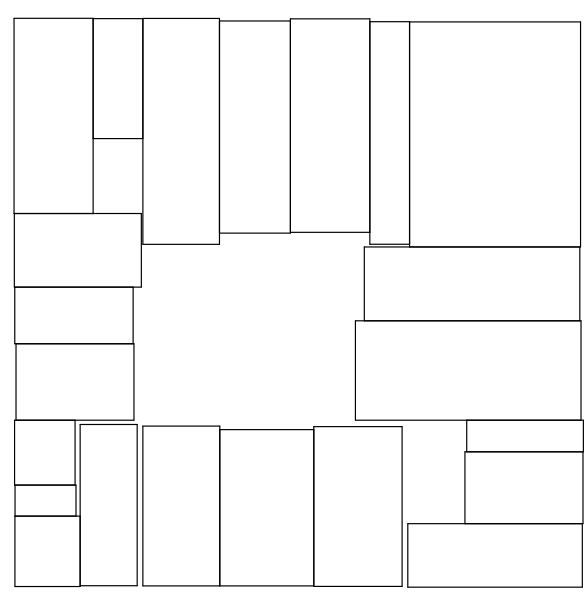

07

\begin{tabular}{|l|r|}
\hline Base Surface Size & $200 \times 200$ metres \\
\hline Width: depth Ratio & 4.0 Maximum \\
\hline Minimum Width Range & $7.5 \mathrm{~m}-20.0 \mathrm{~m}$ \\
\hline Maximum Width Range & $20.0 \mathrm{~m}-45.0 \mathrm{~m}$ \\
\hline
\end{tabular}

Figure 4.21: Compilation of outputs - Standard version

CHAPTER FOUR

A CITY IS NOT A TREE 


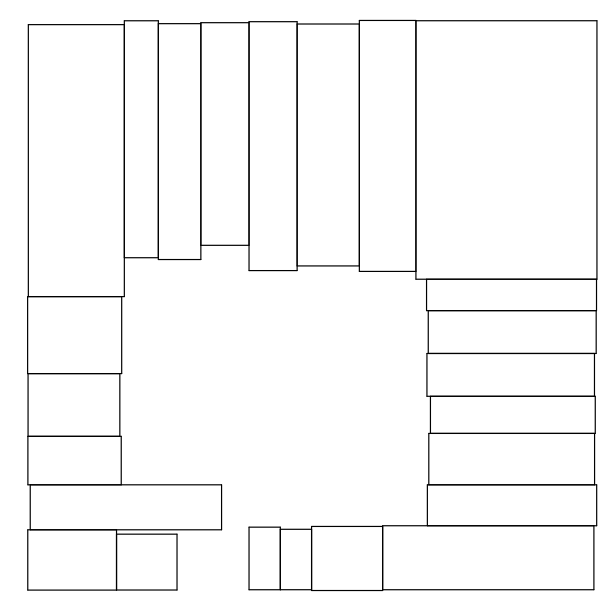

Seed 00

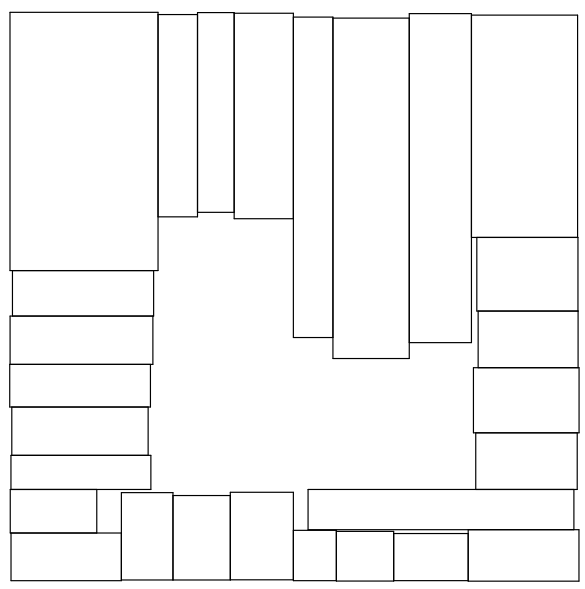

04

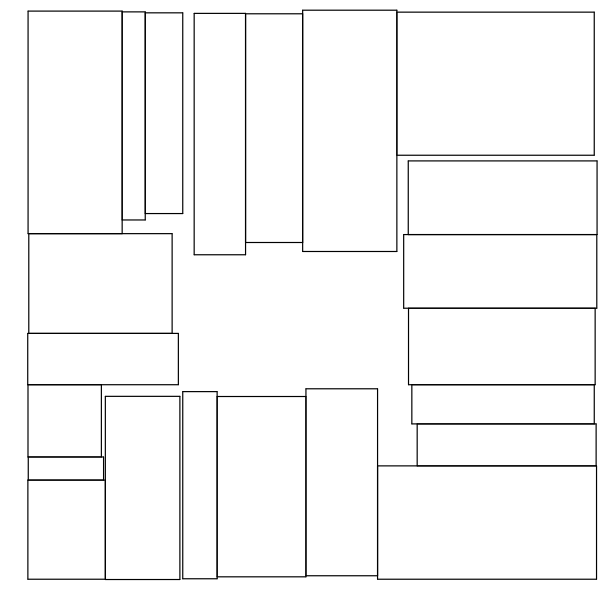

08

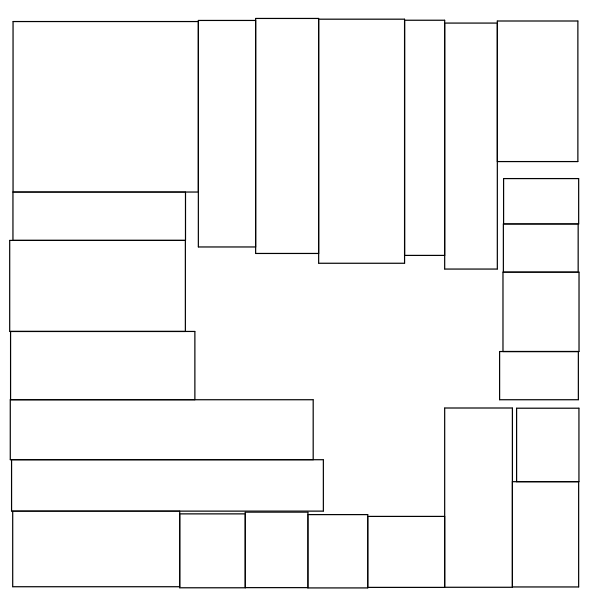

01

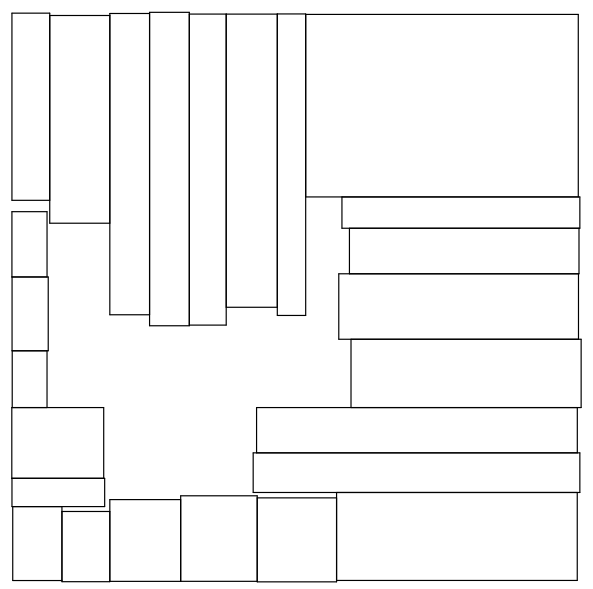

05

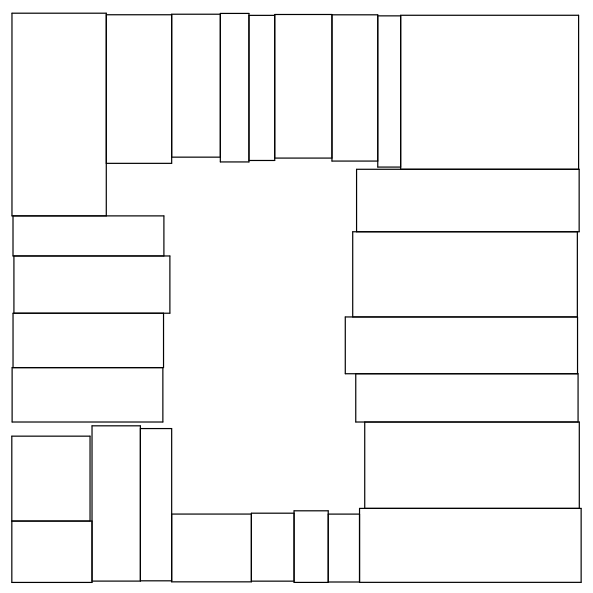

09

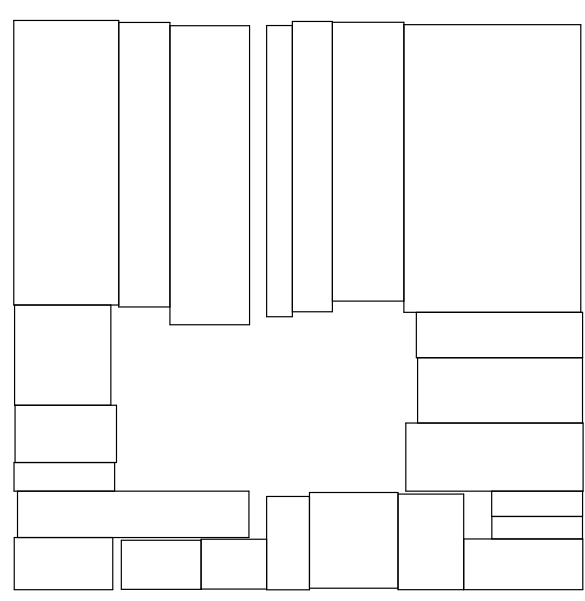

02

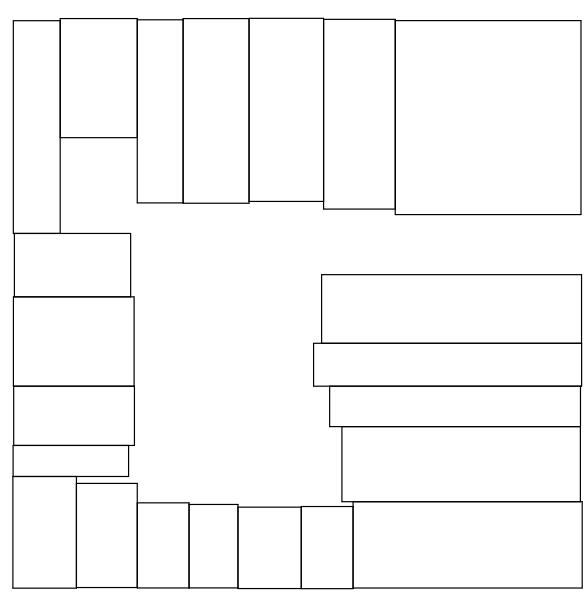

06

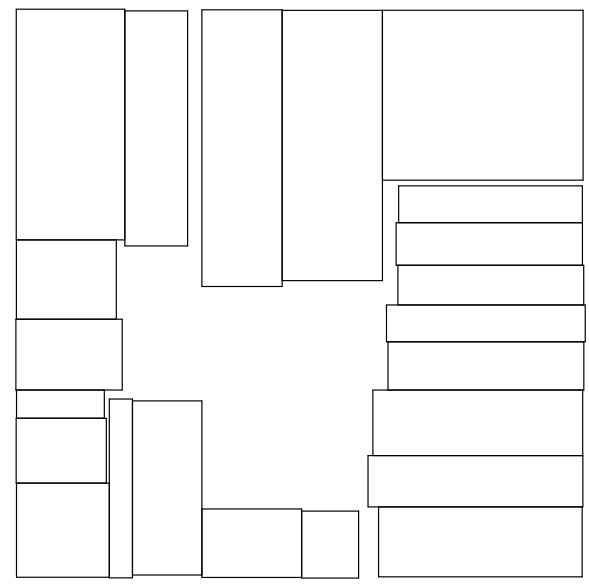

10

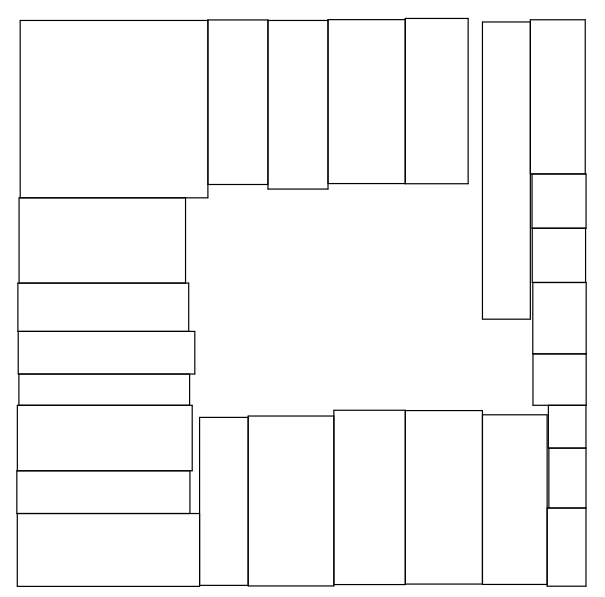

03

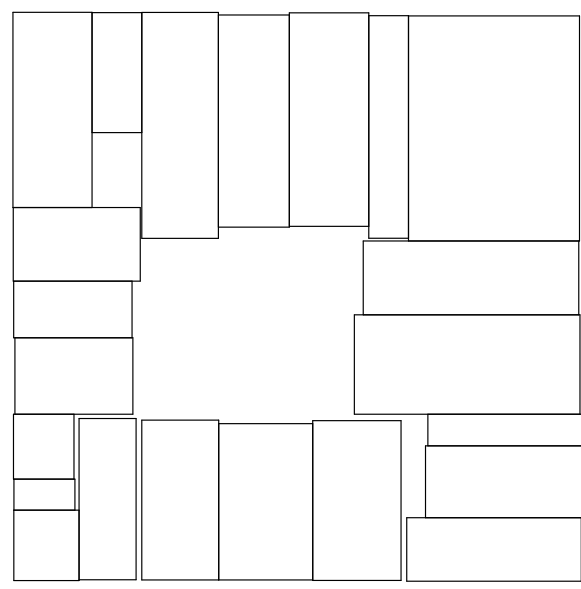

07

\begin{tabular}{|l|r|}
\hline Base Surface Size & $125 \times 125$ metres \\
\hline Width: depth Ratio & 8.0 Maximum \\
\hline Minimum Width Range & $7.5 \mathrm{~m}-20.0 \mathrm{~m}$ \\
\hline Maximum Width Range & $20.0 \mathrm{~m}-45.0 \mathrm{~m}$ \\
\hline
\end{tabular}

Figure 4.22: Compilation of outputs - Increased width: depth ratio 


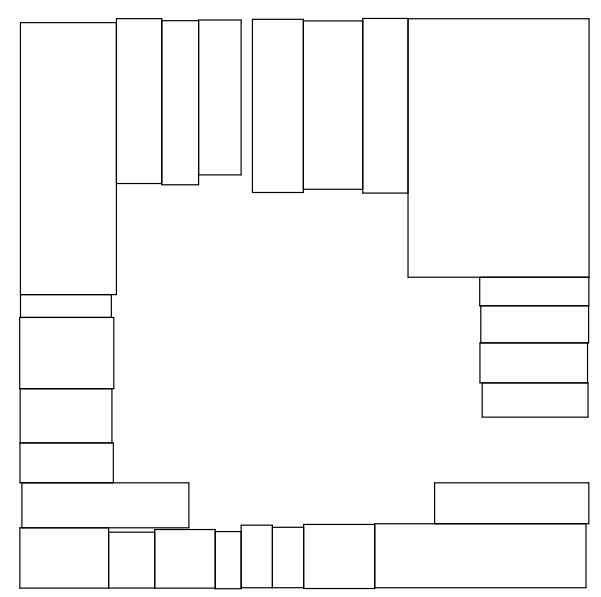

Seed 00

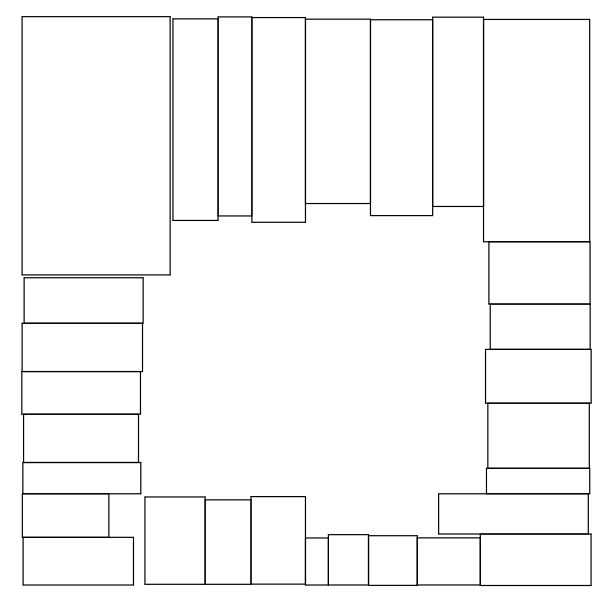

04

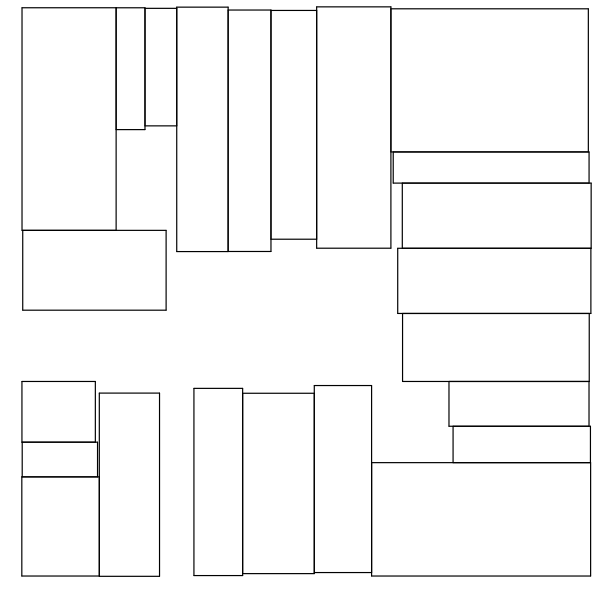

08

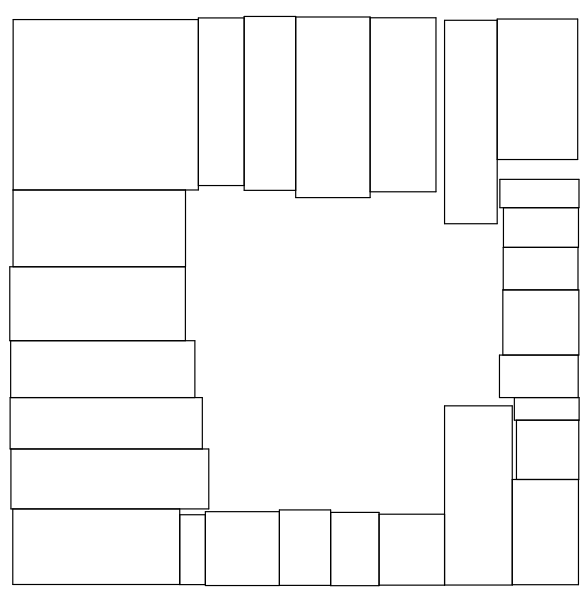

01

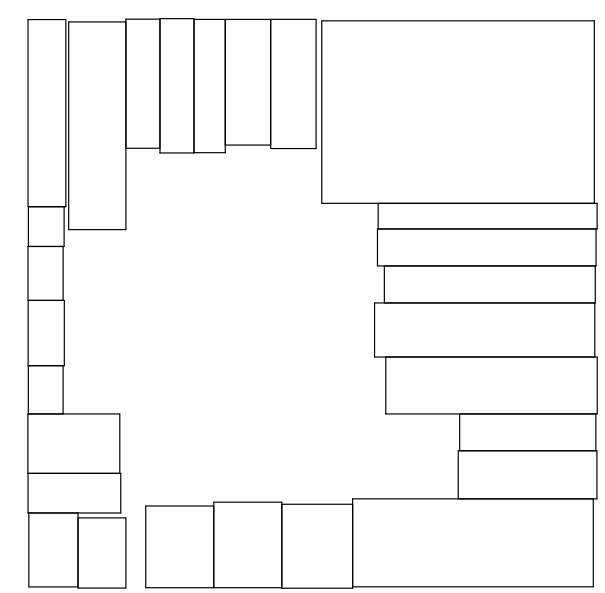

05

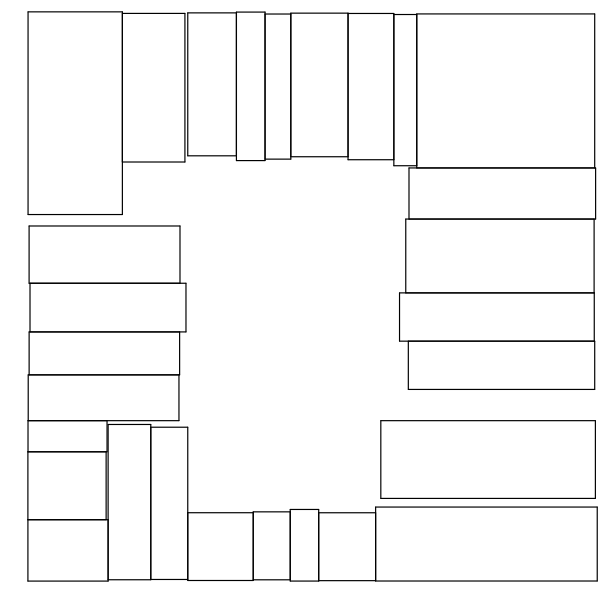

09

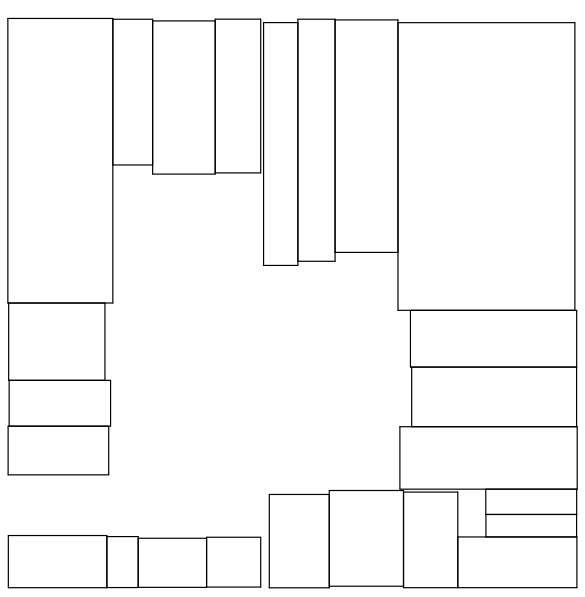

02

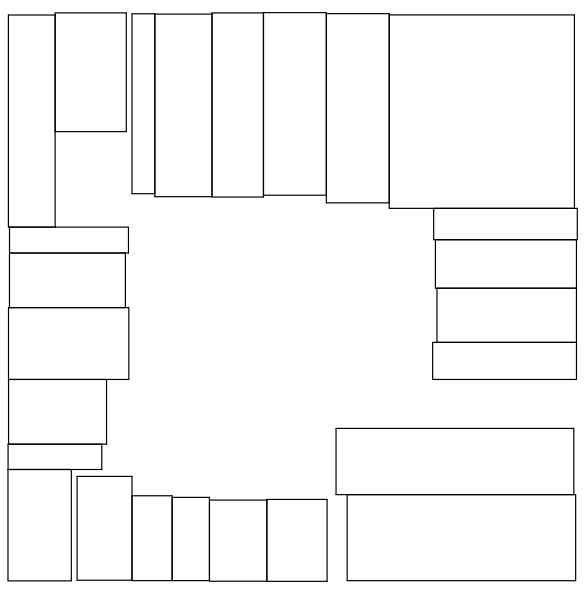

06

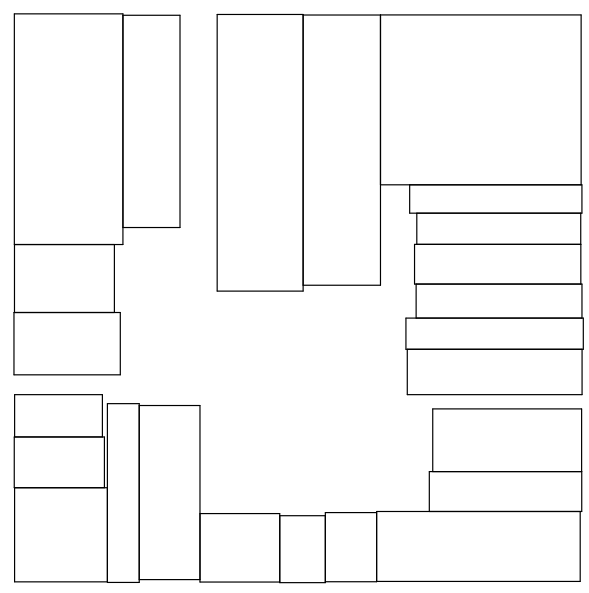

10

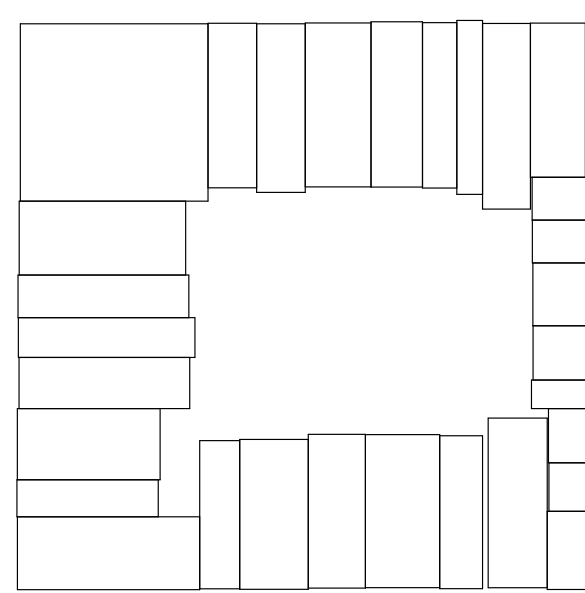

03

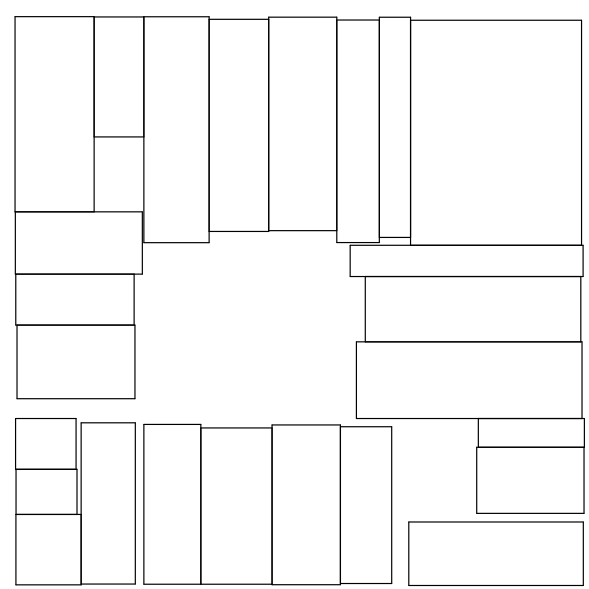

07

\begin{tabular}{|l|r|}
\hline Base Surface Size & $200 \times 200$ metres \\
\hline Width: depth Ratio & 4.0 Maximum \\
\hline Minimum Width Range & $7.5 \mathrm{~m}-15.0 \mathrm{~m}$ \\
\hline Maximum Width Range & $20.0 \mathrm{~m}-35.0 \mathrm{~m}$ \\
\hline
\end{tabular}

Figure 4.23: Compilation of outputs - Reduced minimum / maximum building width

CHAPTER FOUR

A CITY IS NOT A TREE 


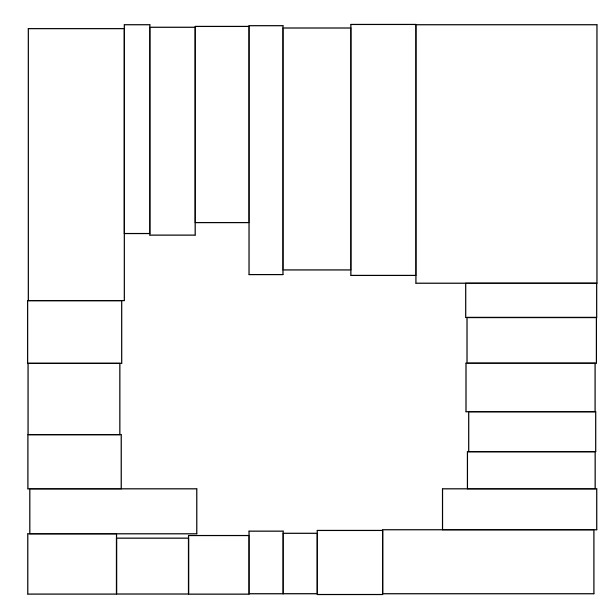

Seed 00

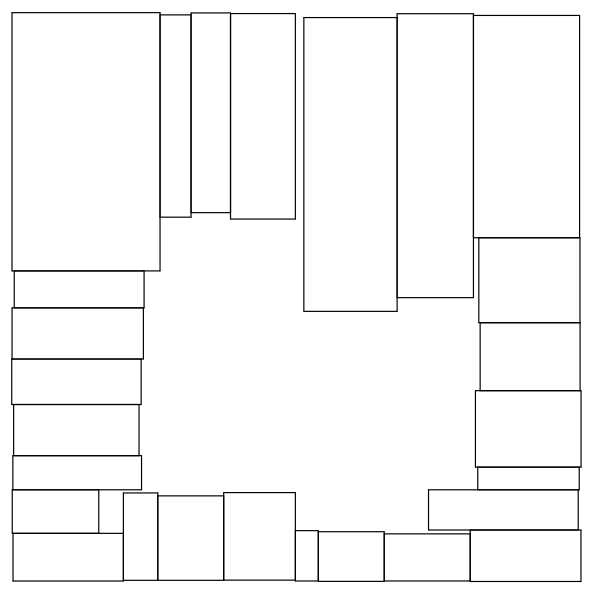

04

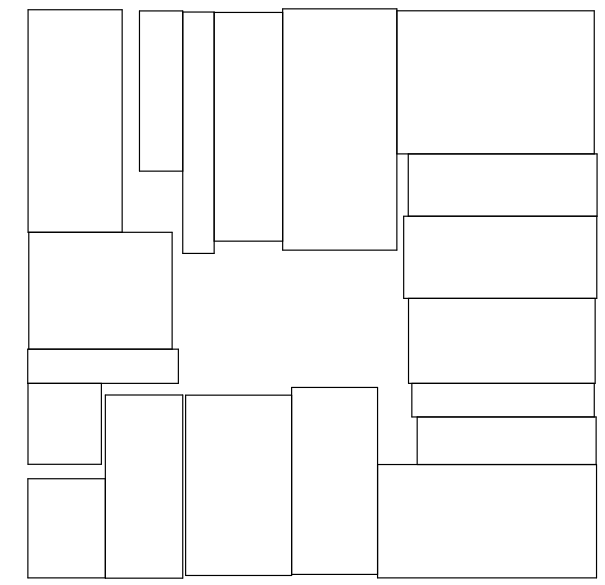

08

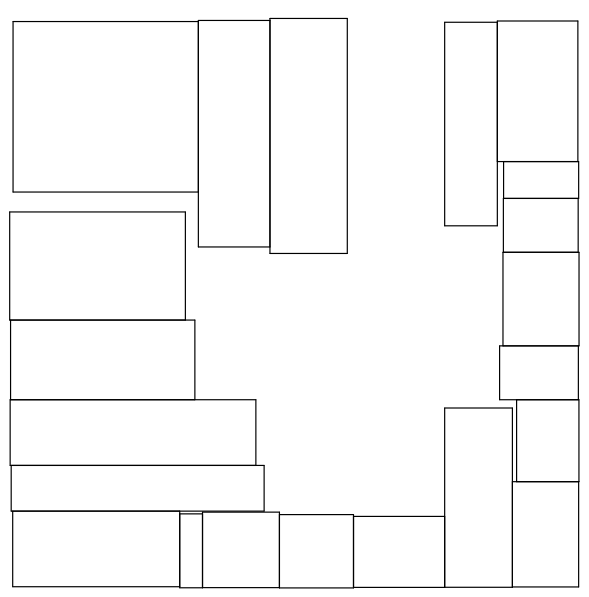

01

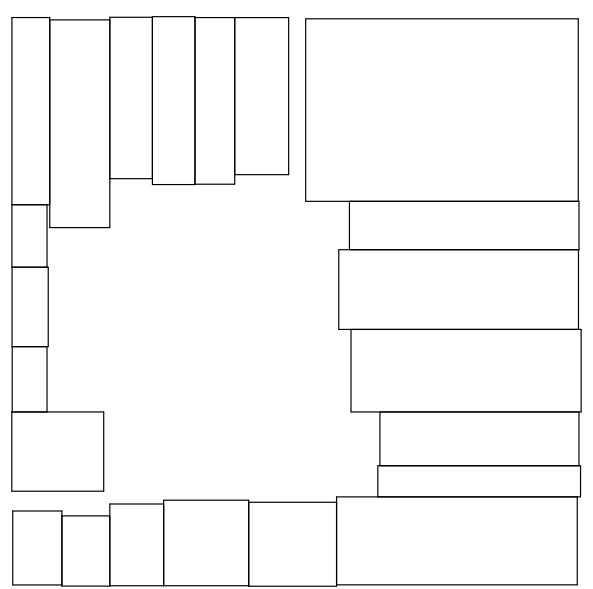

05

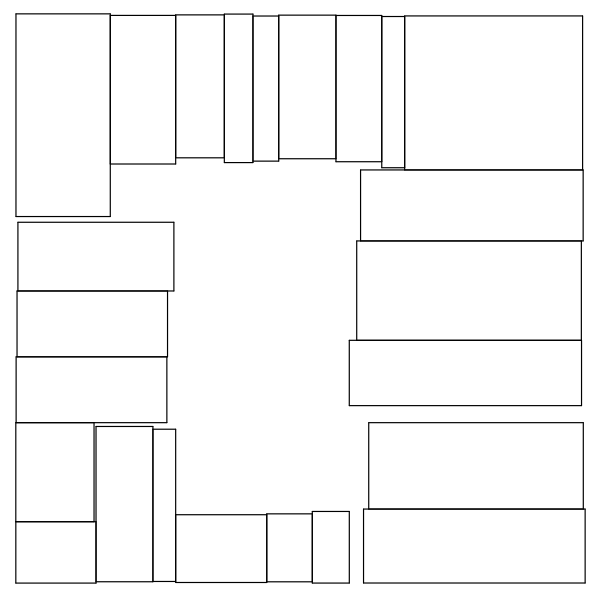

09

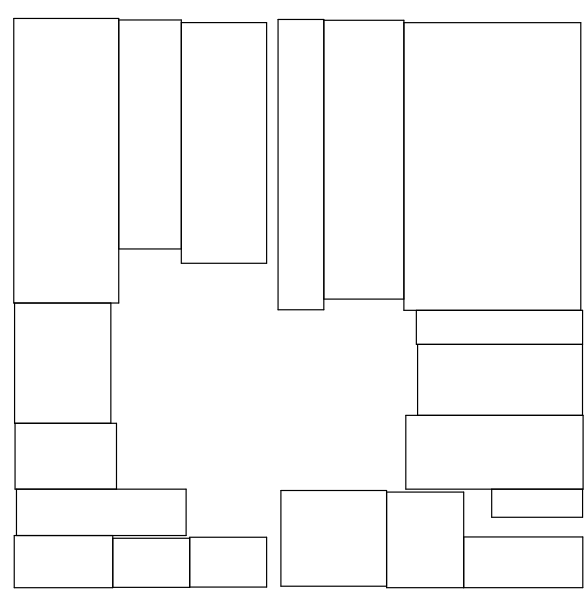

02

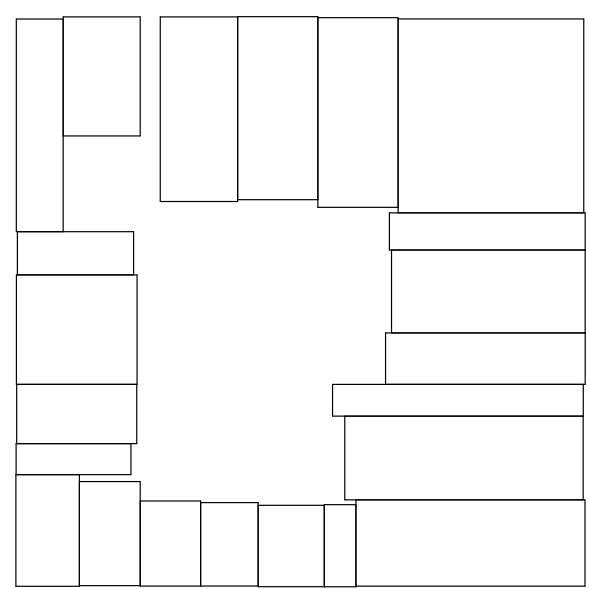

06

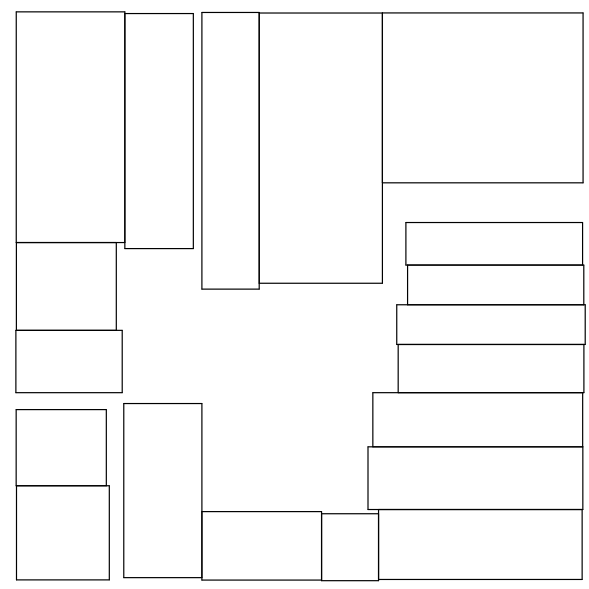

10

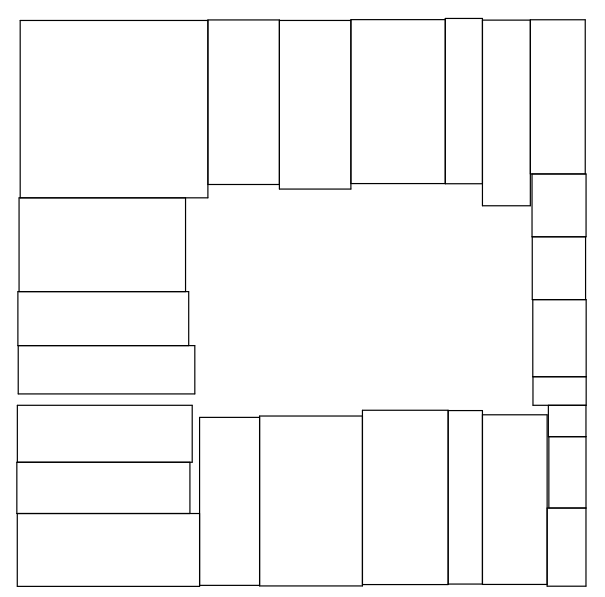

03

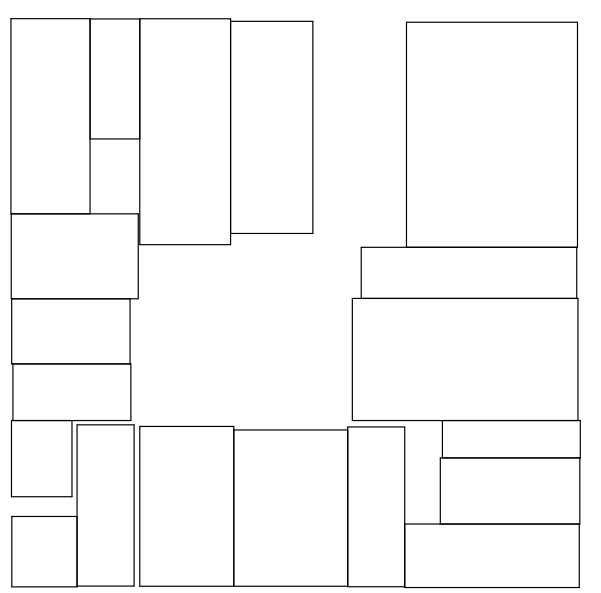

07

\begin{tabular}{|l|r|}
\hline Base Surface Size & $200 \times 200$ metres \\
\hline Width: depth Ratio & 4.0 Maximum \\
\hline Minimum Width Range & $7.5 \mathrm{~m}-25.0 \mathrm{~m}$ \\
\hline Maximum Width Range & $20.0 \mathrm{~m}-55.0 \mathrm{~m}$ \\
\hline
\end{tabular}

Figure 4.24: Compilation of outputs - Increased minimum / maximum building width 


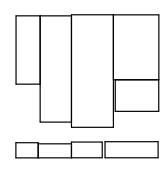

Seed 00

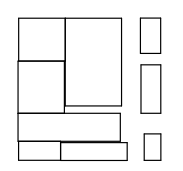

01

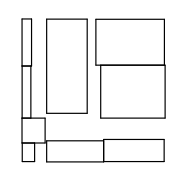

05

04

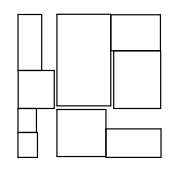

08

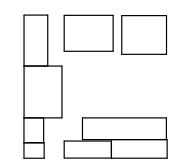

09

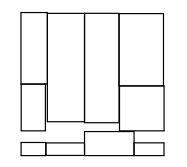

02

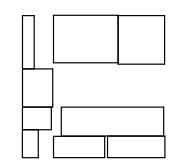

06

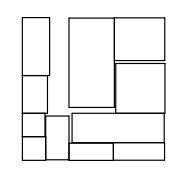

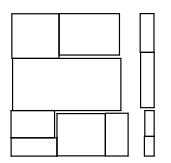

03

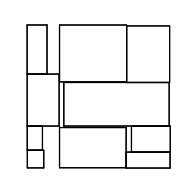

07

\begin{tabular}{|l|r|}
\hline Base Surface Size & $50 \times 50$ metres \\
\hline Width: depth Ratio & 4.0 Maximum \\
\hline Minimum Width Range & $7.5 \mathrm{~m}-15.0 \mathrm{~m}$ \\
\hline Maximum Width Range & $20.0 \mathrm{~m}-35.0 \mathrm{~m}$ \\
\hline
\end{tabular}

Figure 4.25: Compilation of outputs - Significantly decreased surface size

CHAPTER FOUR

A CITY IS NOT A TREE 


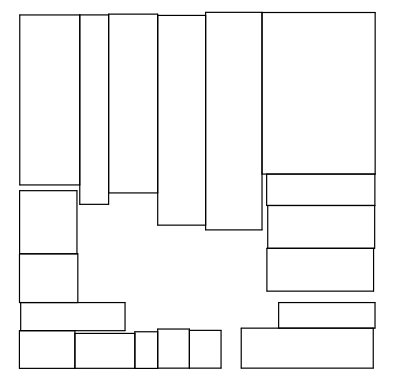

Seed 00

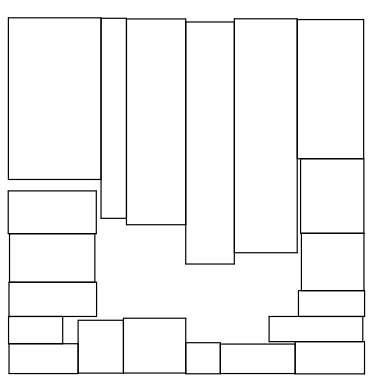

04

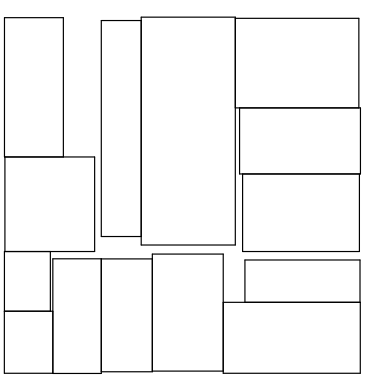

08

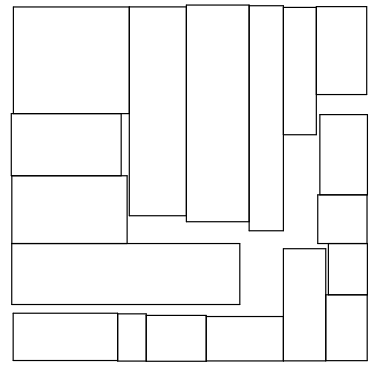

01

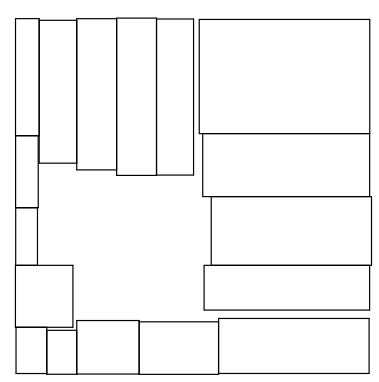

05

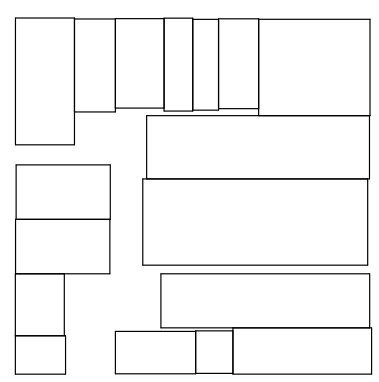

09

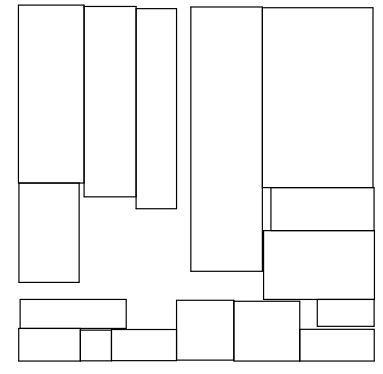

02

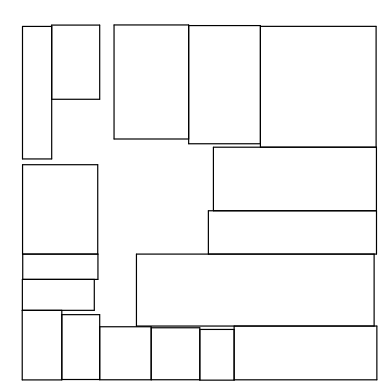

06

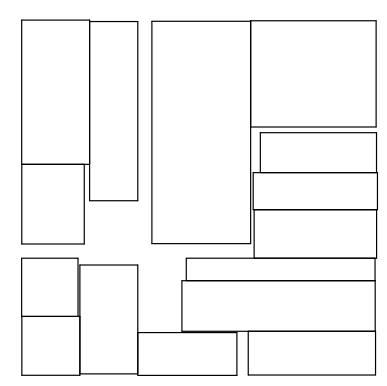

10

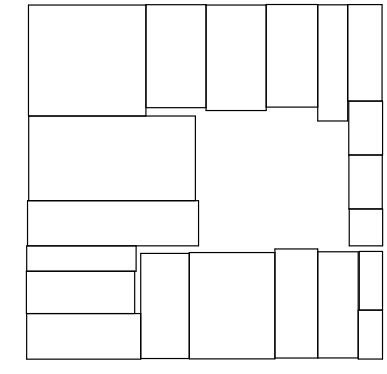

03

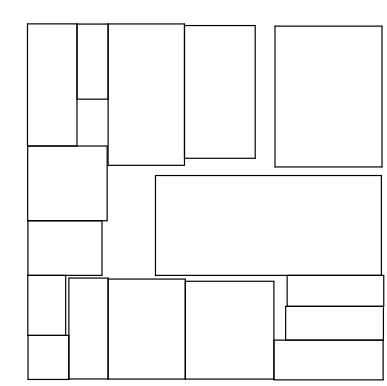

07

Figure 4.26: Compilation of outputs - Decreased surface size

\begin{tabular}{|l|r|}
\hline Base Surface Size & $125 \times 125$ metres \\
\hline Width: depth Ratio & 4.0 Maximum \\
\hline Minimum Width Range & $7.5 \mathrm{~m}-25.0 \mathrm{~m}$ \\
\hline Maximum Width Range & $20.0 \mathrm{~m}-55.0 \mathrm{~m}$ \\
\hline
\end{tabular}




\section{BUILDING WIDTHS}

Regarding design bias, one concern is the designer specifically controlling the widths of any given block's building footprints. To avoid this, the user specifies a width range, comprised of an absolute minimum and maximum value. Once these values are specified, RNG decides what the minimum and maximum width values for each block will be. This additional step further prevents each block from forming in the same manner as the neighbouring blocks, or footprints. Ideally, researched figures would inform this range, rather than an approximation and or anecdotal information.

To produce more accurate/predictable the designer could employ a fixed value, rather than a range of values. However, there are very few situations in which this would be truly necessary. Within the context of this research, fixed values operate solely during testing phases, for the export of final images, or when locating specific issues within the various systems.

\section{BUILDING WIDTH: DEPTH RATIO}

The building width: depth ratio houses similar issues relating to design bias. The first inclusion of this ratio was in the fourth iteration of the building footprint generator, at which point the system utilised a single value of 4 . This value was selected for it is aesthetic qualities and appropriate outputs at a variety of scales. Larger values failed on smaller base surfaces, whereas smaller values failed on larger base surfaces.

In the hopes of achieving greater variety in the city blocks, the maximum ratio value shifted from being a single value to a range. This was done to work in the same way that the building widths have been allowed to. The result of this system's implementation would be seen in the resultant diversity of building footprint outputs, and the more organic appearance of the constructed spaces. 


\section{CHAPTER FIVE \\ CITY BLOCK EXTRUSION}




\section{EXTRUDED BLOCK GENERATOR}

The nature of this series of investigations is that it will never truly be complete. There is no limit to the amount of detail, built elements, optimisation, or other improvements that could become integrated with the system. However, as mentioned earlier in the research, aiming for increased optimisation and efficiency can be an arduous process, with rapidly diminishing returns. Operations such as boolean unions and region intersections are computationally intensive and have often been the bottleneck in these systems. However, as with any normal learning process, exposure results in understanding and a reduction in the likelihood of repeating the same mistake.

At work of this scale the utilisation of $2 \mathrm{D}$ geometry is significantly more efficient than its 3D counterpart. The downside to relying on 2D geometries is that the built forms can become more basic. As a result of this approach the generated geometry could not be used in an environment requiring accurate modelling for shadows, textures, and so forth. As stated earlier in this research, the solution to this 'problem' is to treat the outputs as a base. From this point detail can be added as and where necessary in other modelling software(s). 


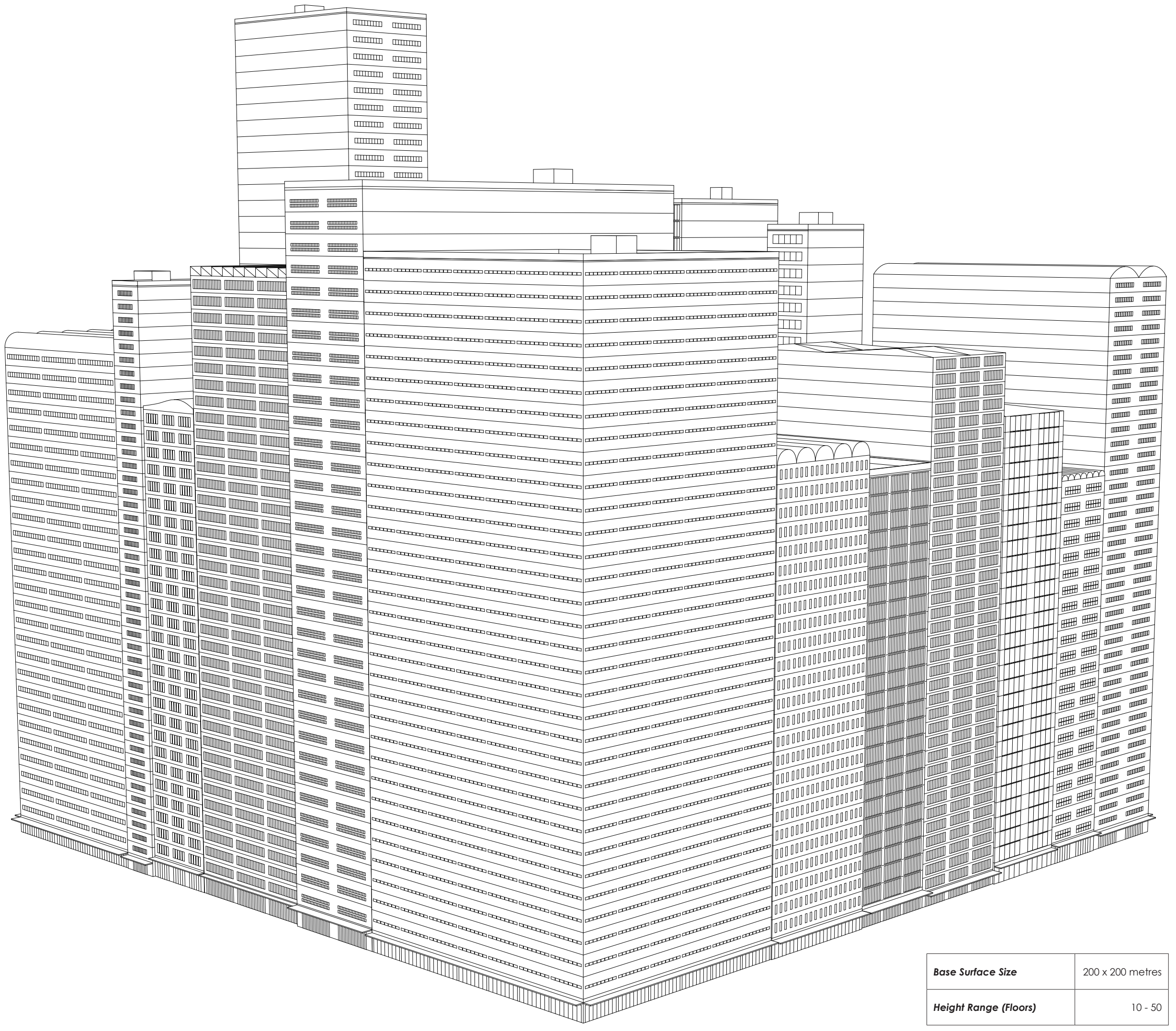

Figure 5.01: Single output from extruded block generator, Seed 10 


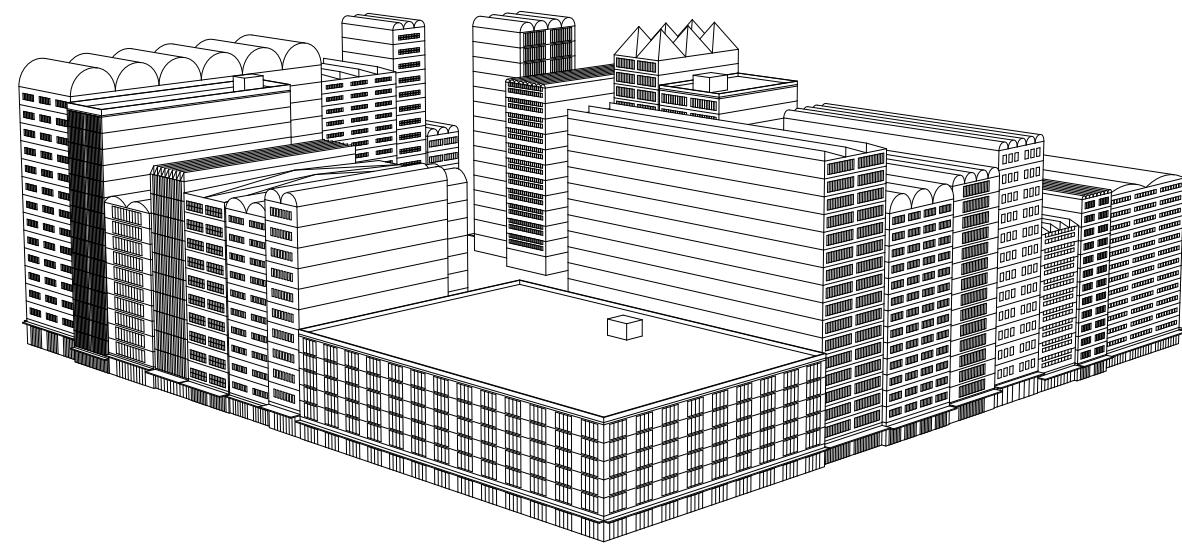

Seed 00

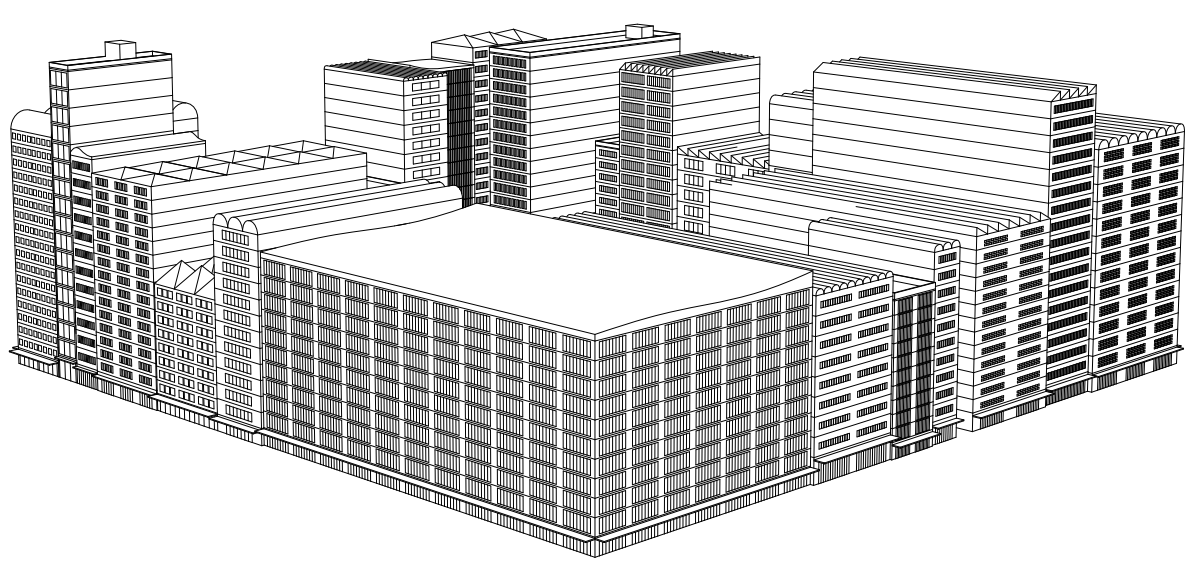

02

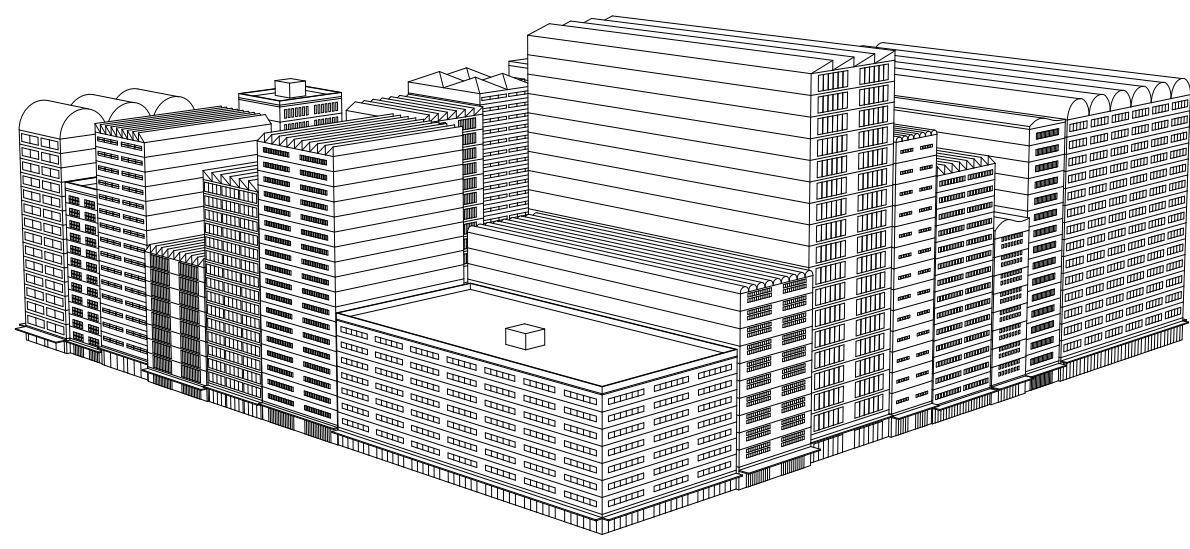

04

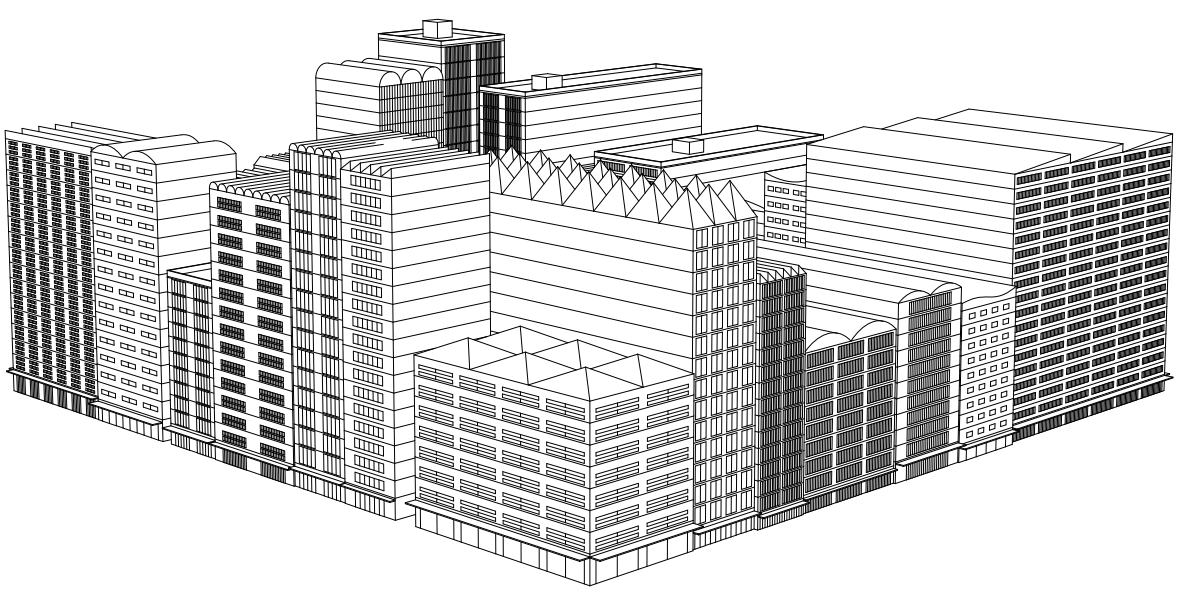

01

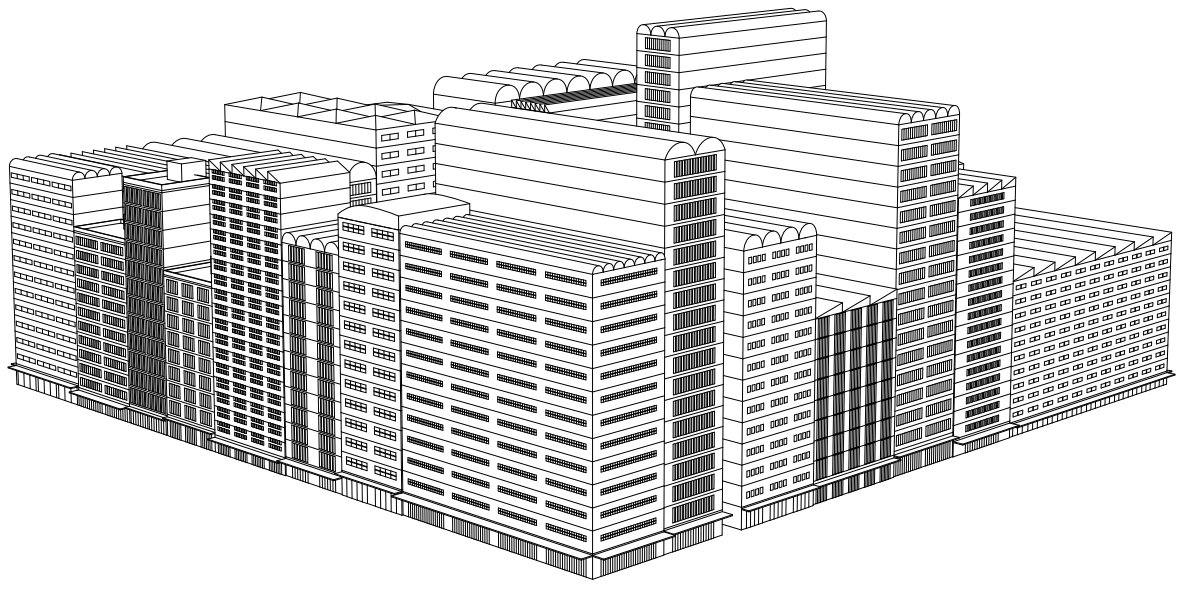

03

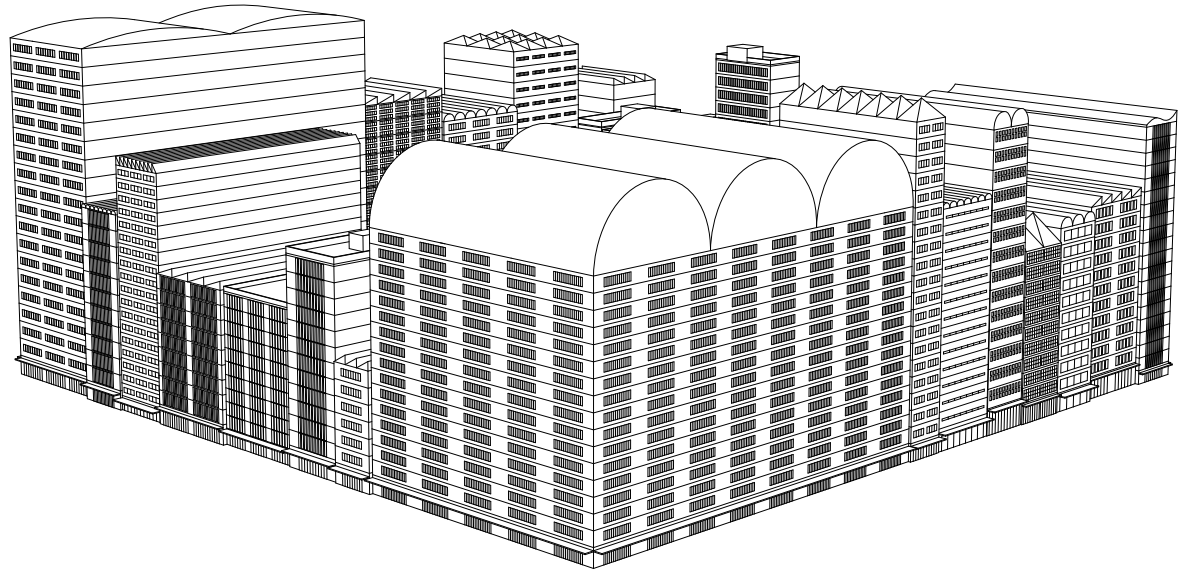

05

Figure 5.02: Compilation of Extruded Block Generator outputs

CHAPTER FIVE

A CITY IS NOT A TREE 


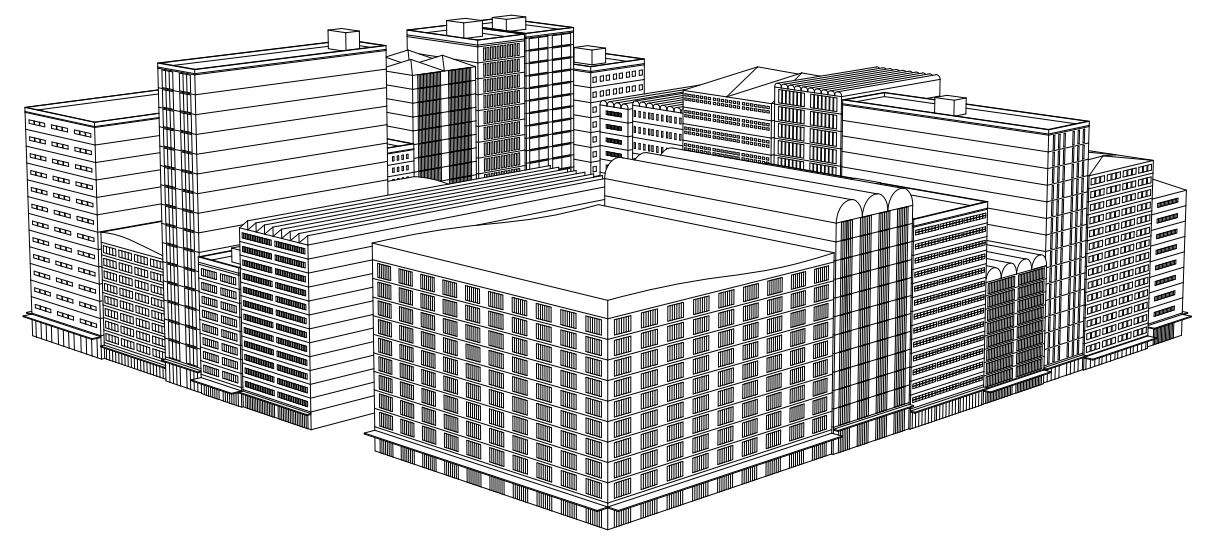

06

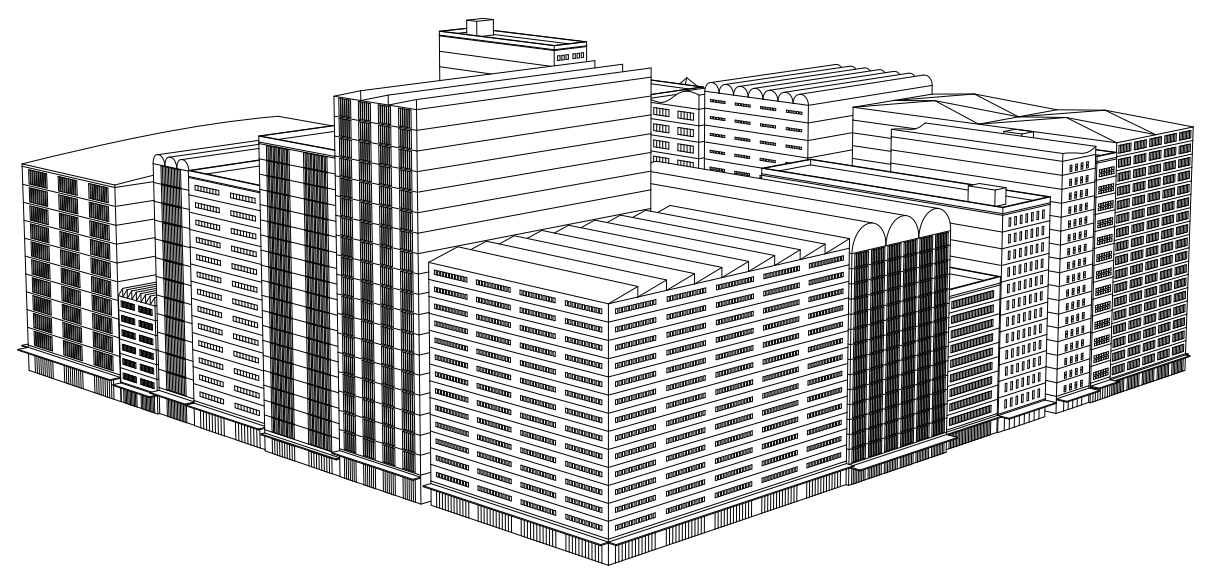

08

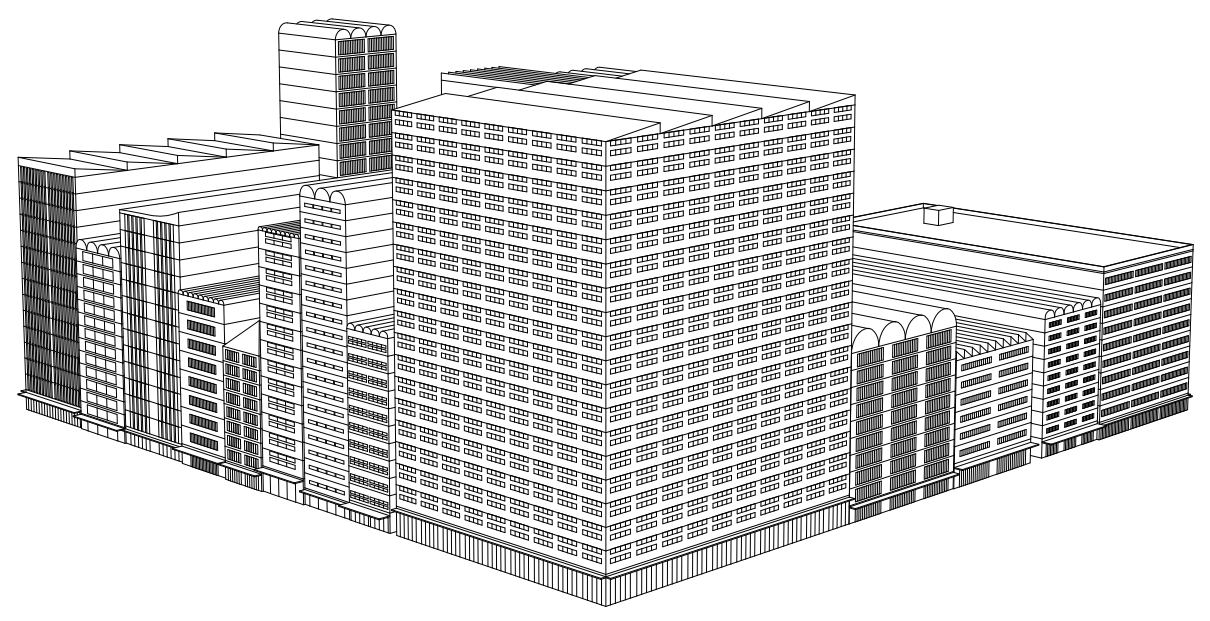

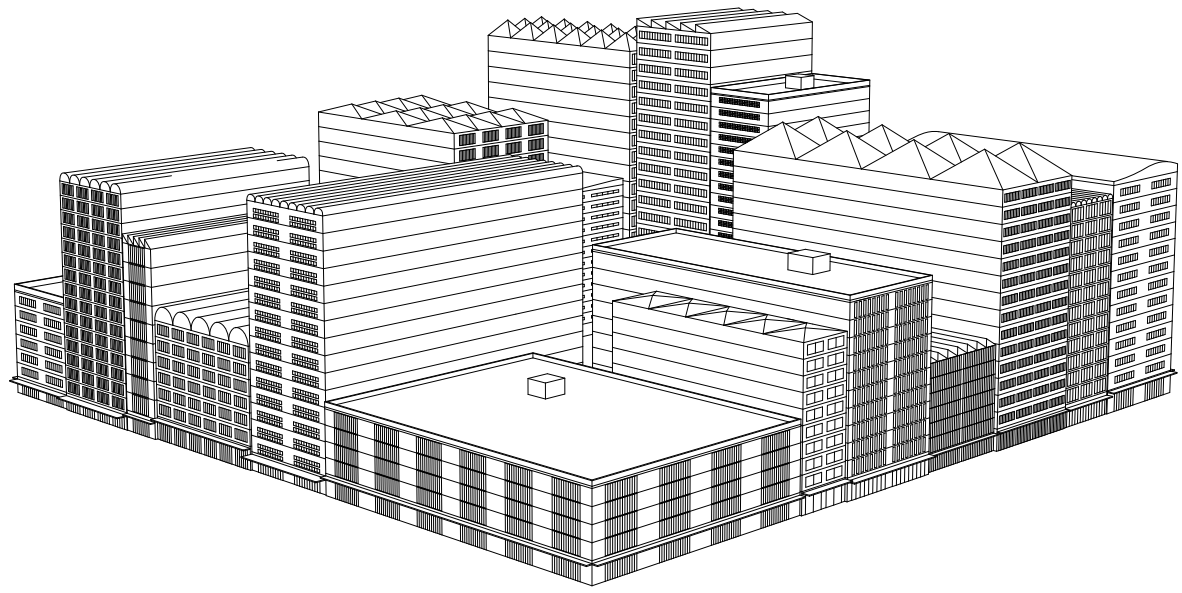

07

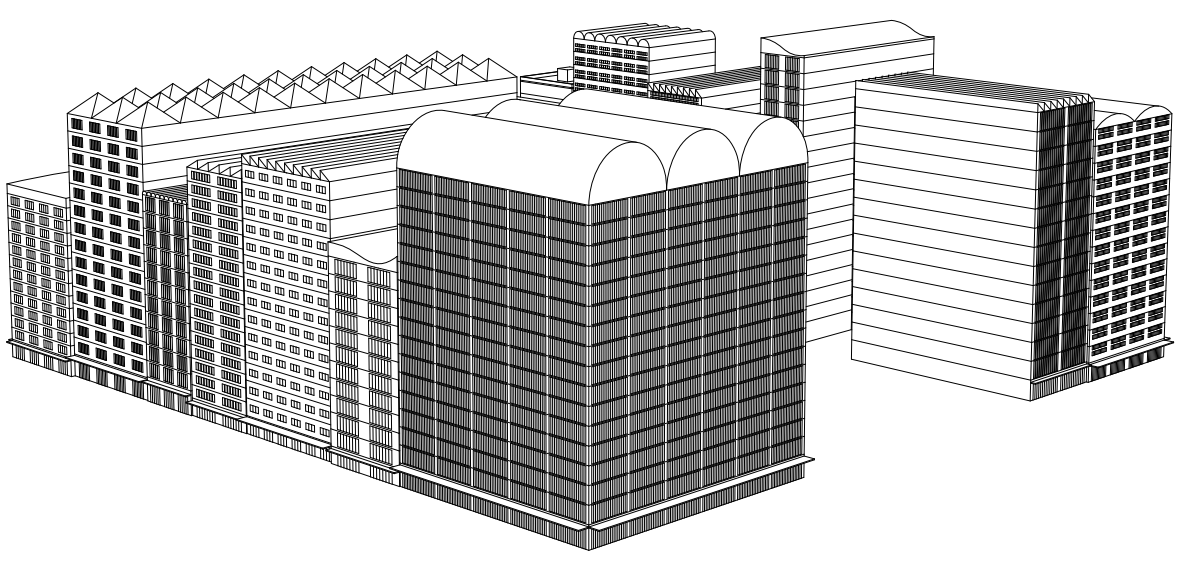

09

10

\begin{tabular}{|l|r|}
\hline Floor Range & $4-20$ \\
\hline Floor Height Range & $3.0-4.5$ \\
\hline Minimum Building Width / Depth & 15 \\
\hline Ground Floor Multiplication Range & $1.0-1.5$ \\
\hline
\end{tabular}

Figure 5.02: Compilation of Extruded Block Generator outputs (cont.) 


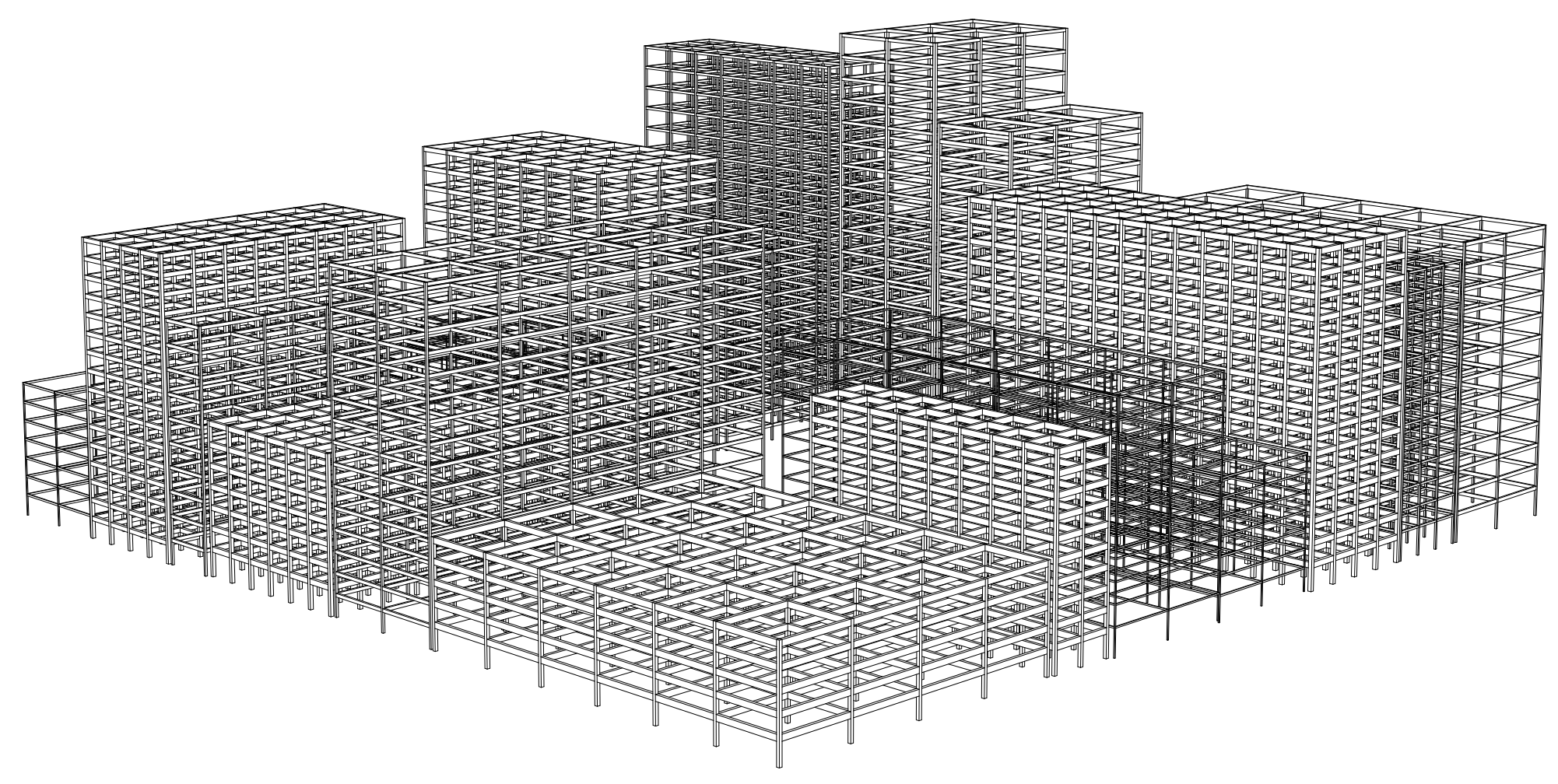

Figure 5.03: Anatomy of a block: Structure, Seed 07

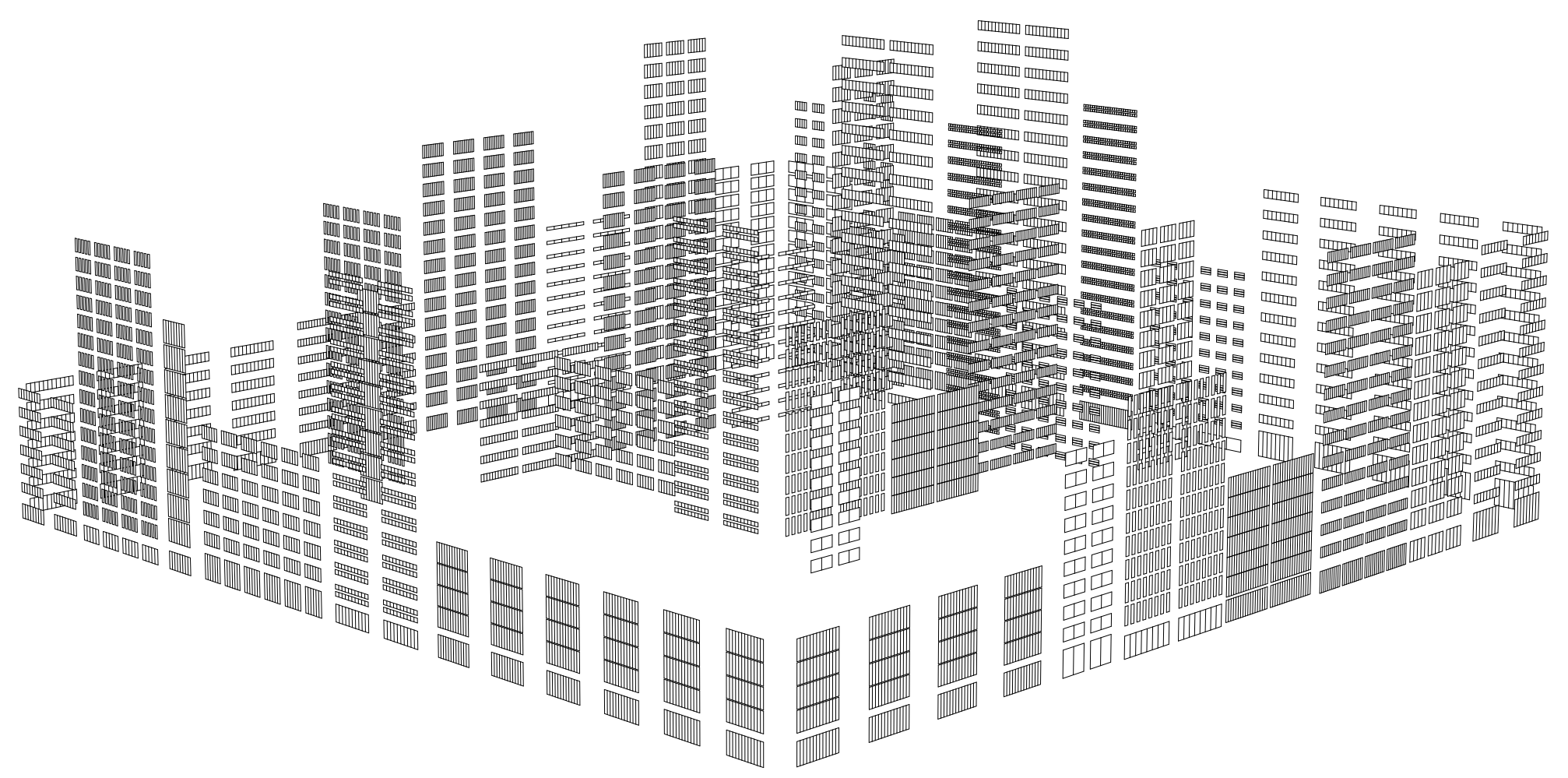

Figure 5.04: Windows

CHAPTER FIVE

A CITY IS NOT A TREE 


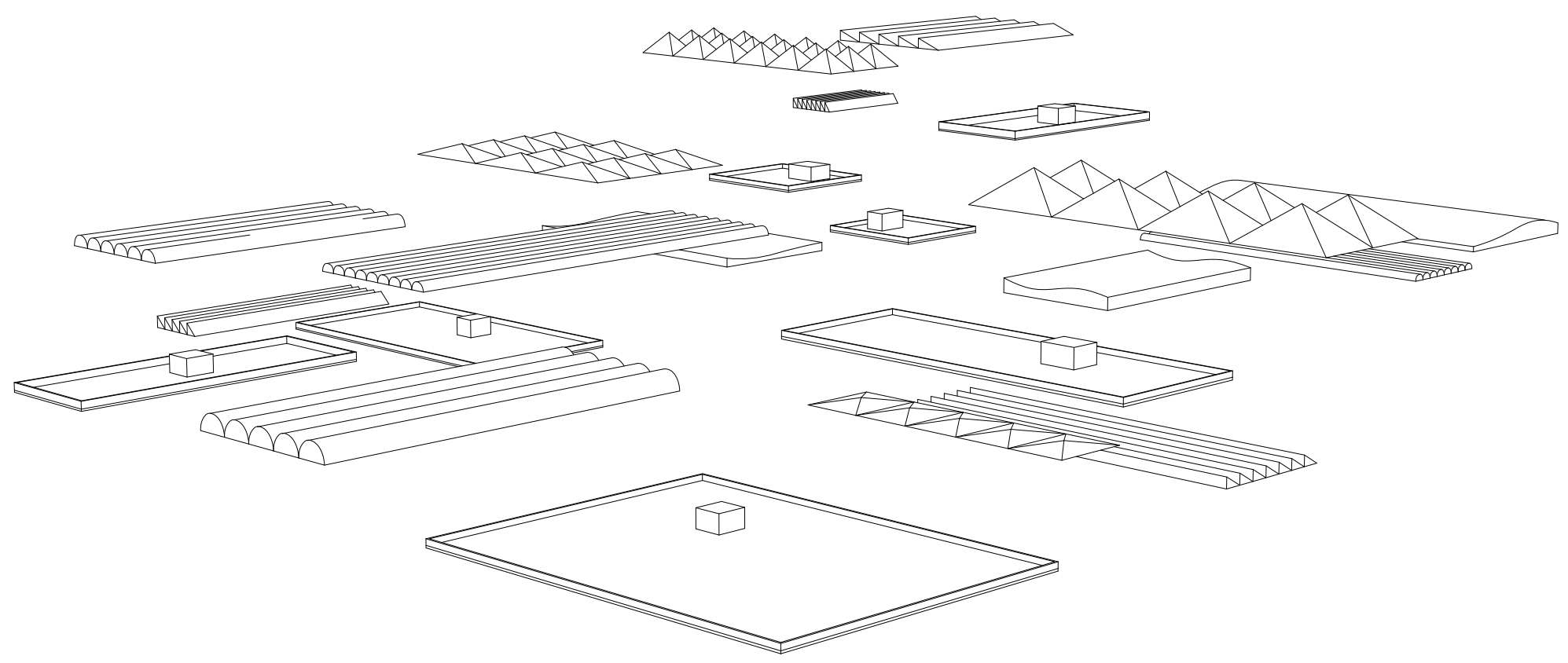

Figure 5.05: Rooftops

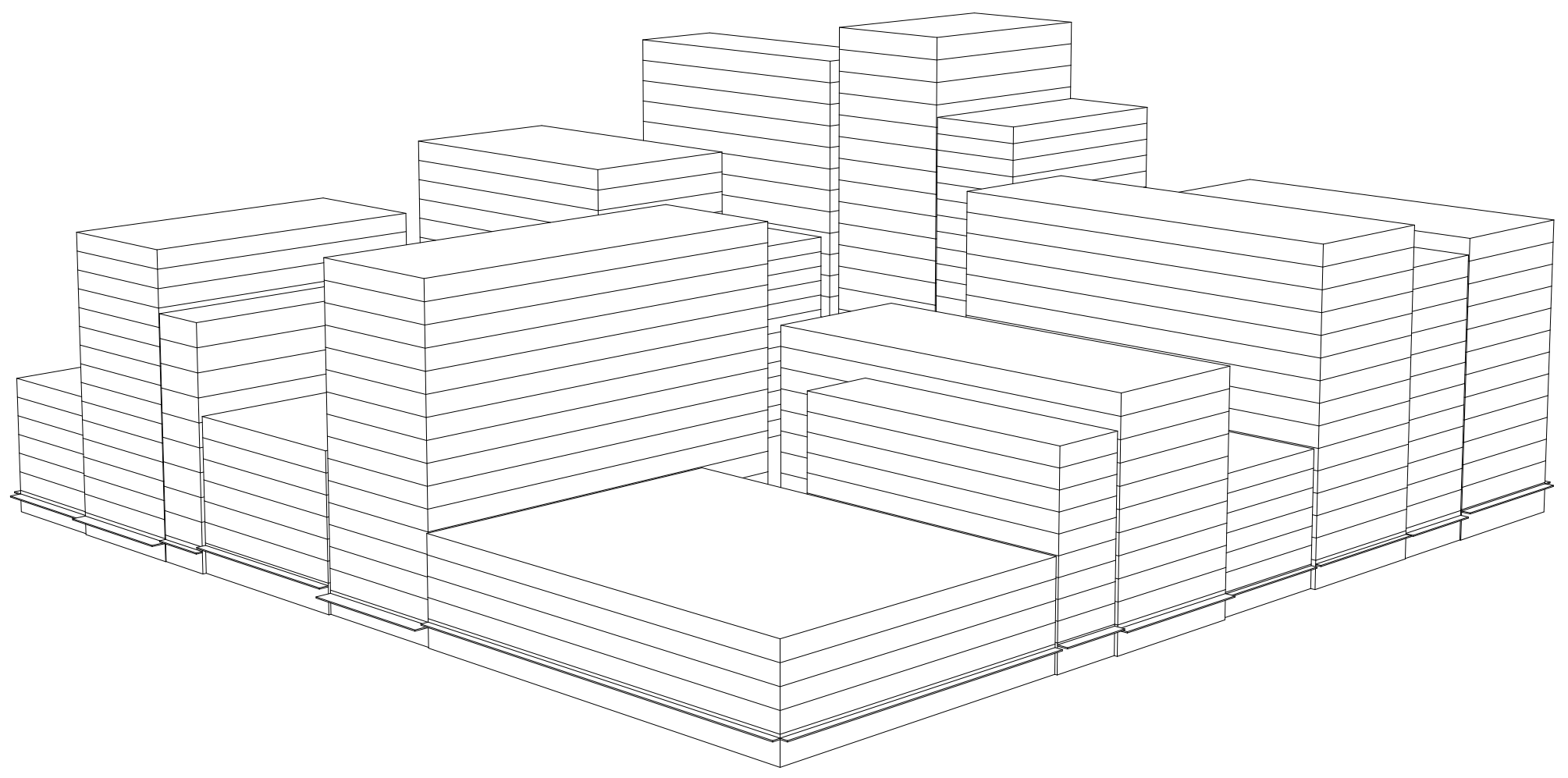

Figure 5.06: Building Volumes \& Verandahs

page 086 


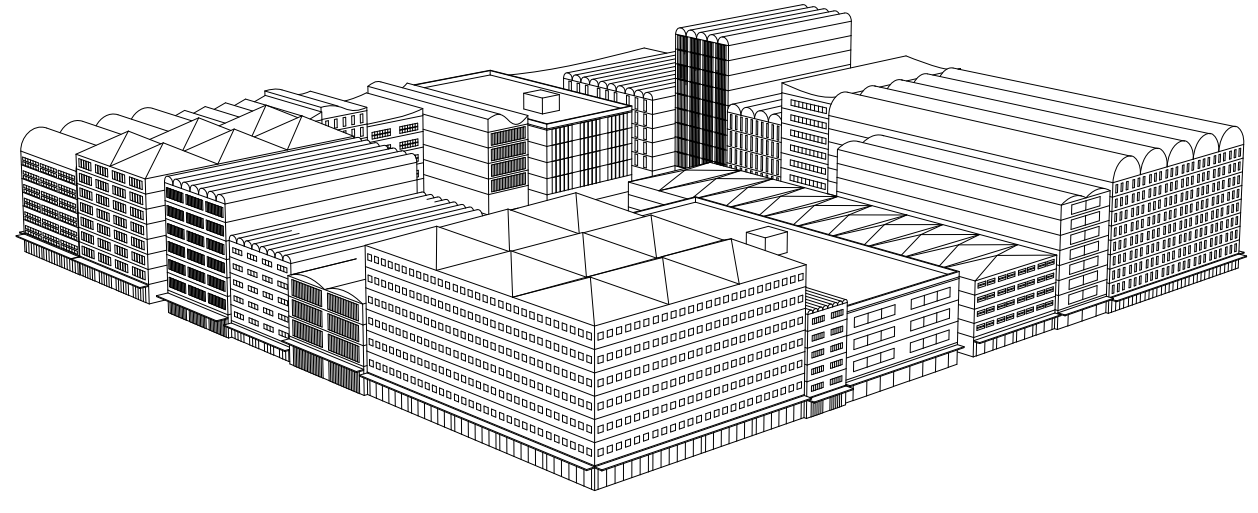

2 - 10 Floors

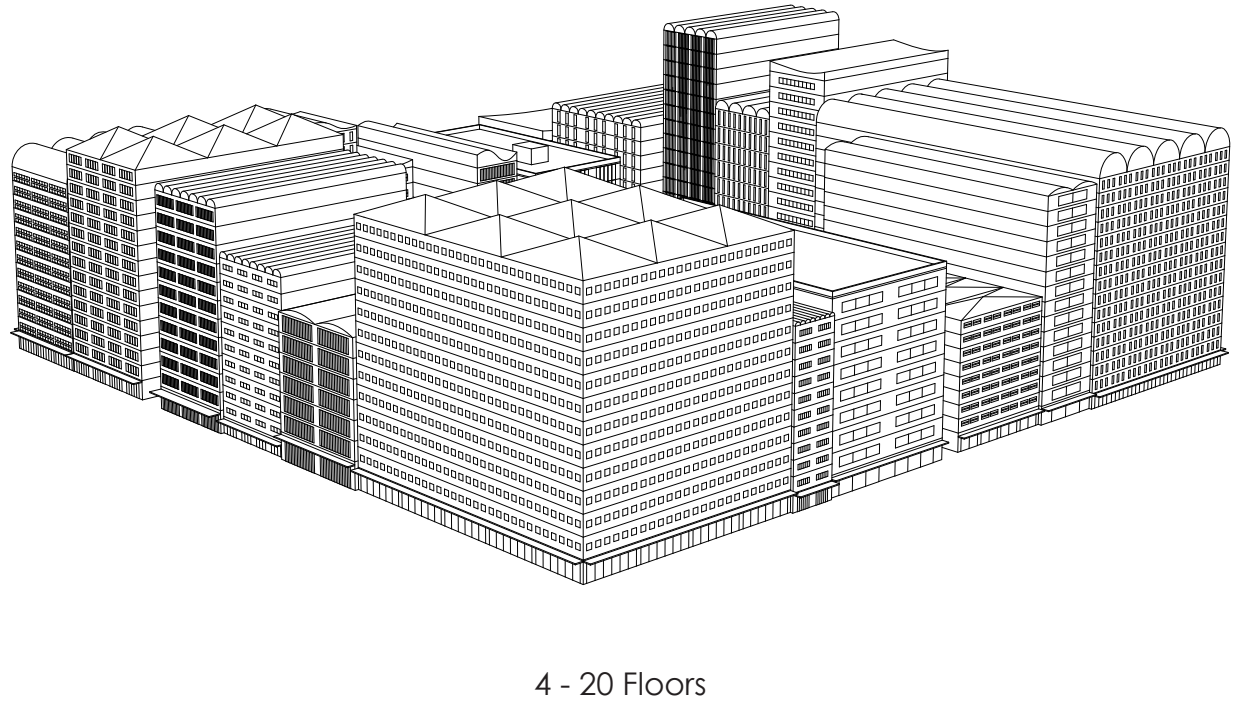

4 - 20 Floors 


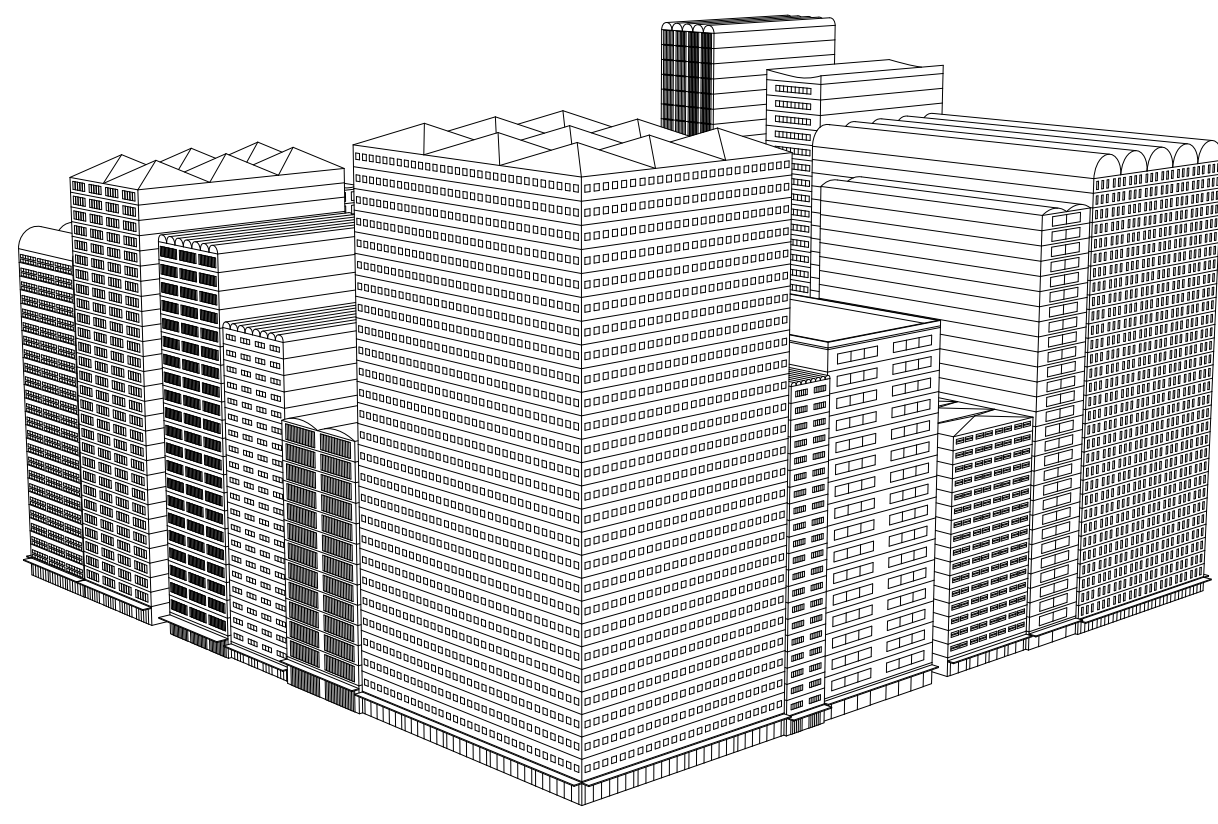

8 - 40 Floors

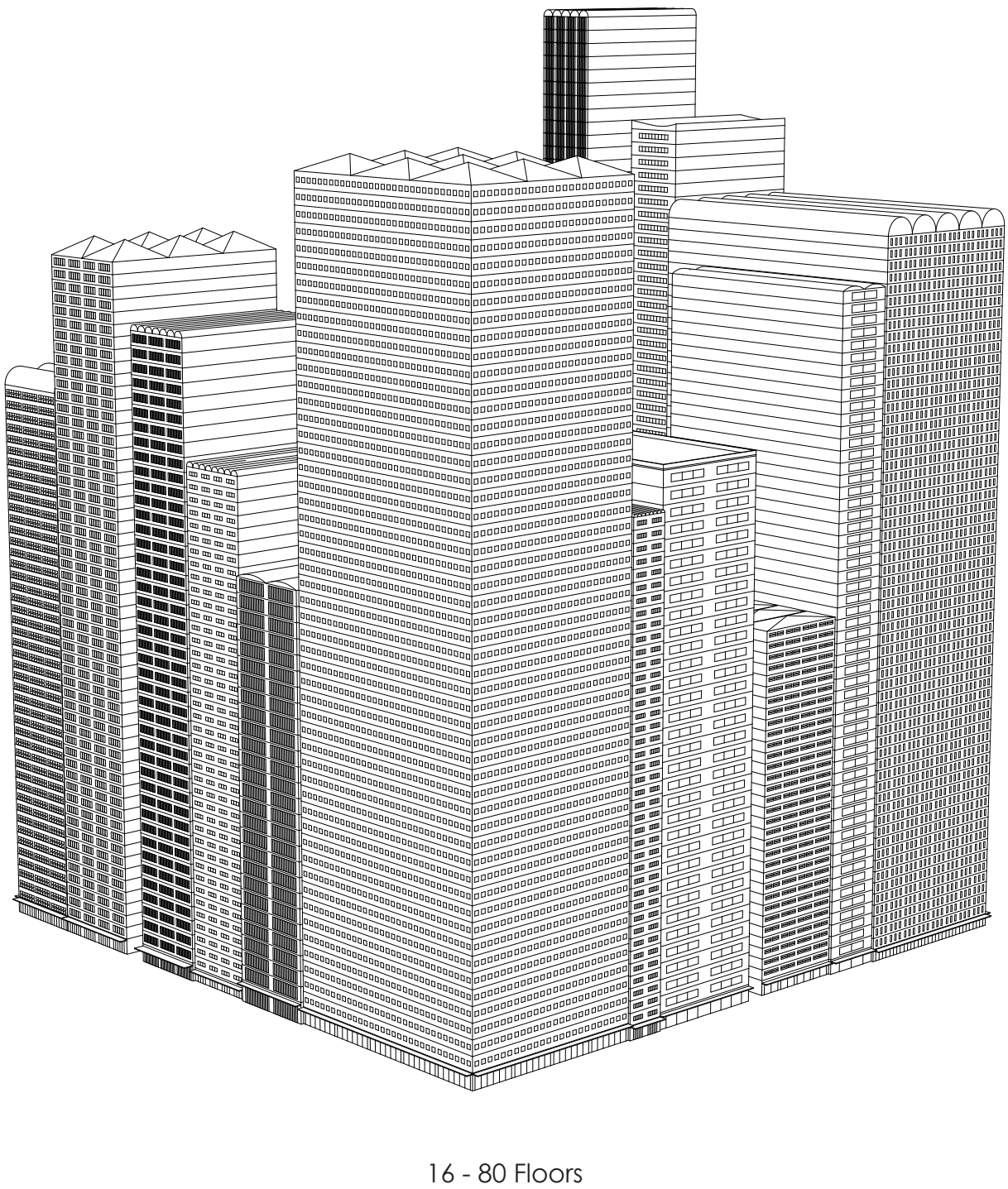

$16-80$ Floors 


\section{SMALL SCALE CITY GENERATOR}

The act of generating a neighbourhood was an extension of generating an individual city block. At its most basic stage, this system involved multiple repetitions of the process used to create an individual city block.

Because Grasshopper is generating each of these blocks simultaneously the process takes significantly longer. Instead of the $\sim 10$ seconds taken to produce a single outcome, this system can be expected to take around 1 minute, or longer, to produce the resultant work.

This investigation has highlighted the need to construct a neighbourhood, or city, as a whole. The process also highlights the necessity for optimisations to the system. Regarding constructing the block as a whole, each block should respond to those around it. Enacting this change would allow for a far greater articulation of the neighbourhood as a whole, as well as creating a more organic push and pull dynamic.

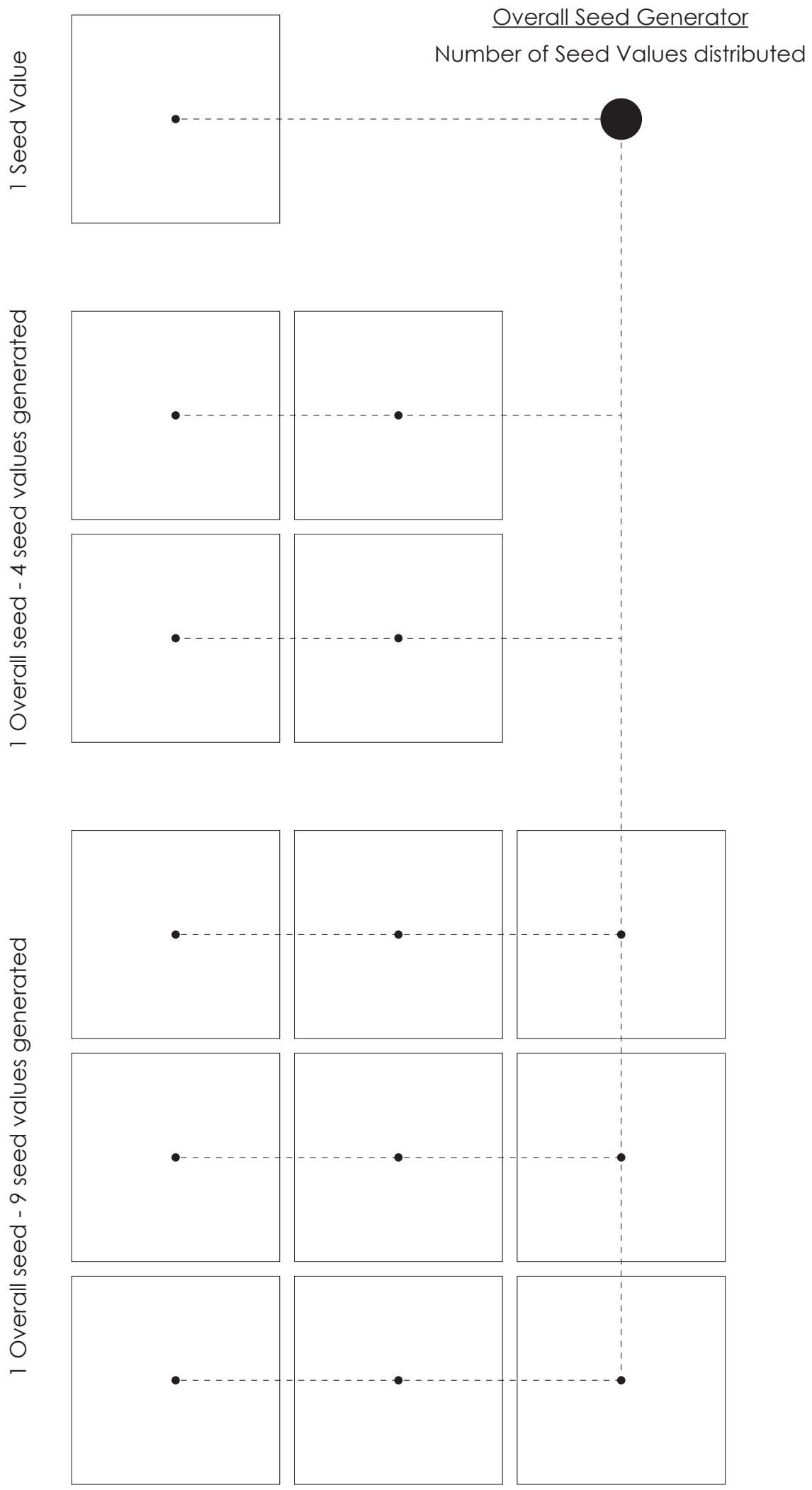

Figure 5.08: Small scale city generator seed distribution 


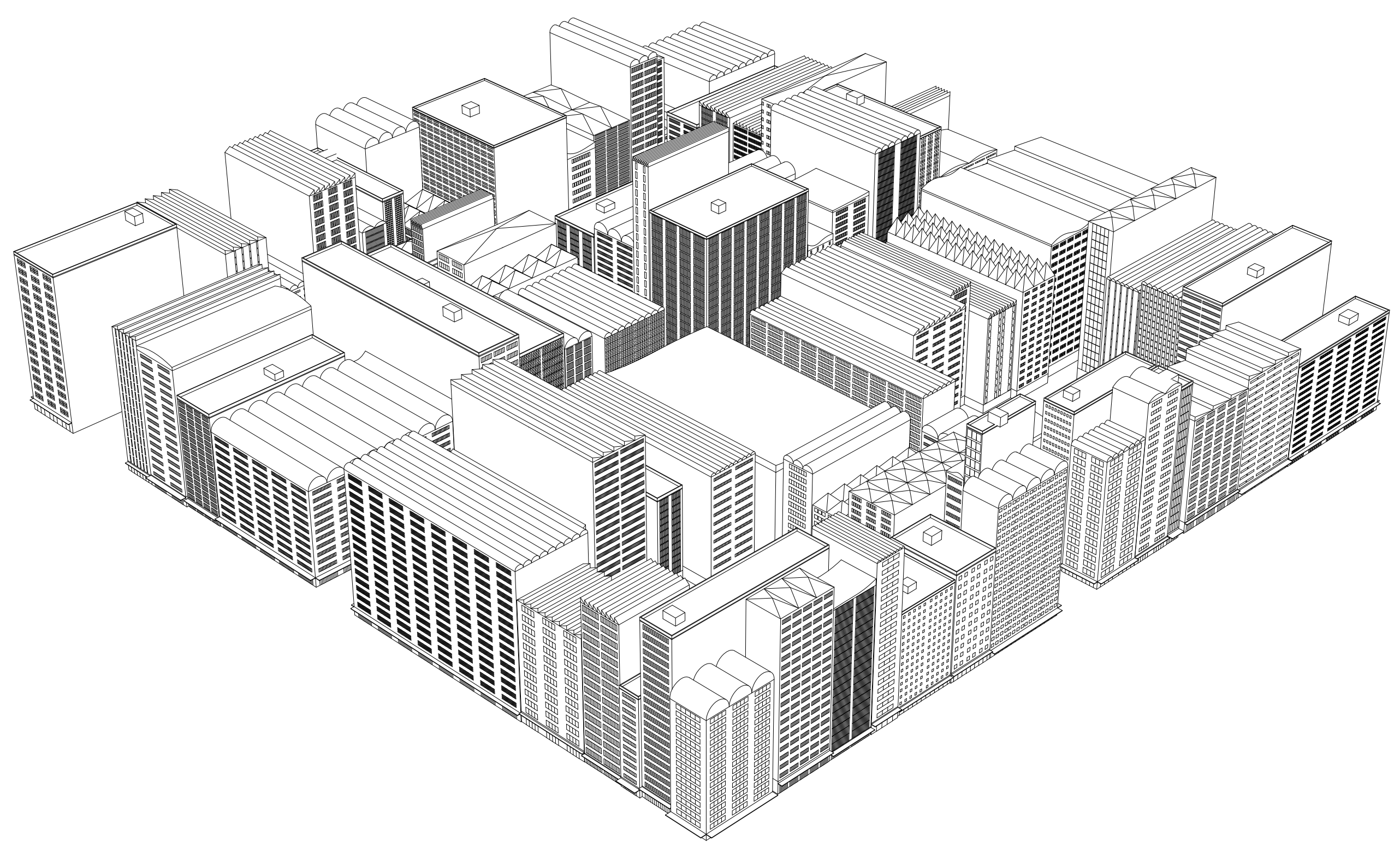

Figure 5.09: Small scale city generator output, Seed 02 


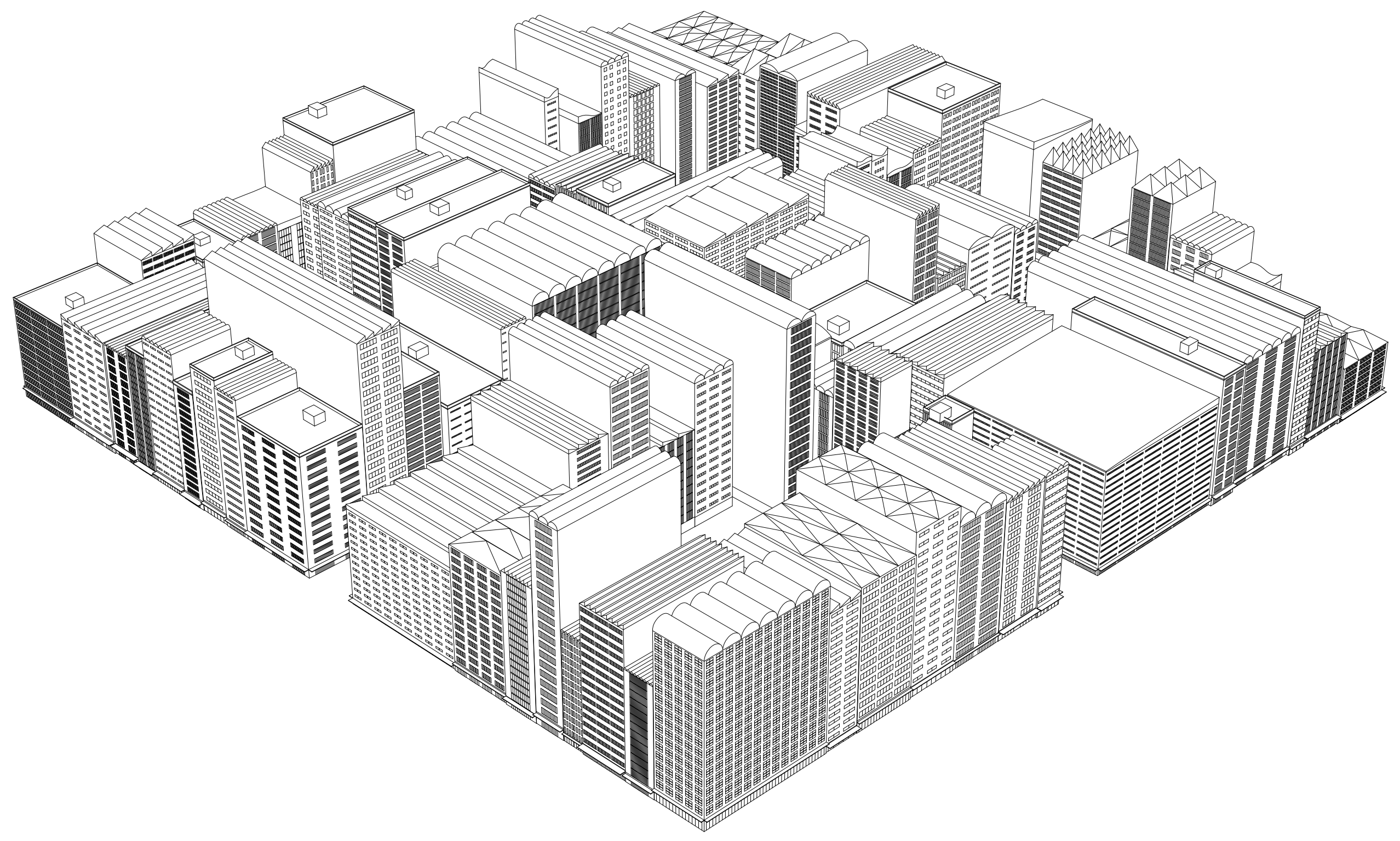

Figure 5.10: Small scale city generator output, $2 \times 2$ Blocks, Seed 03 


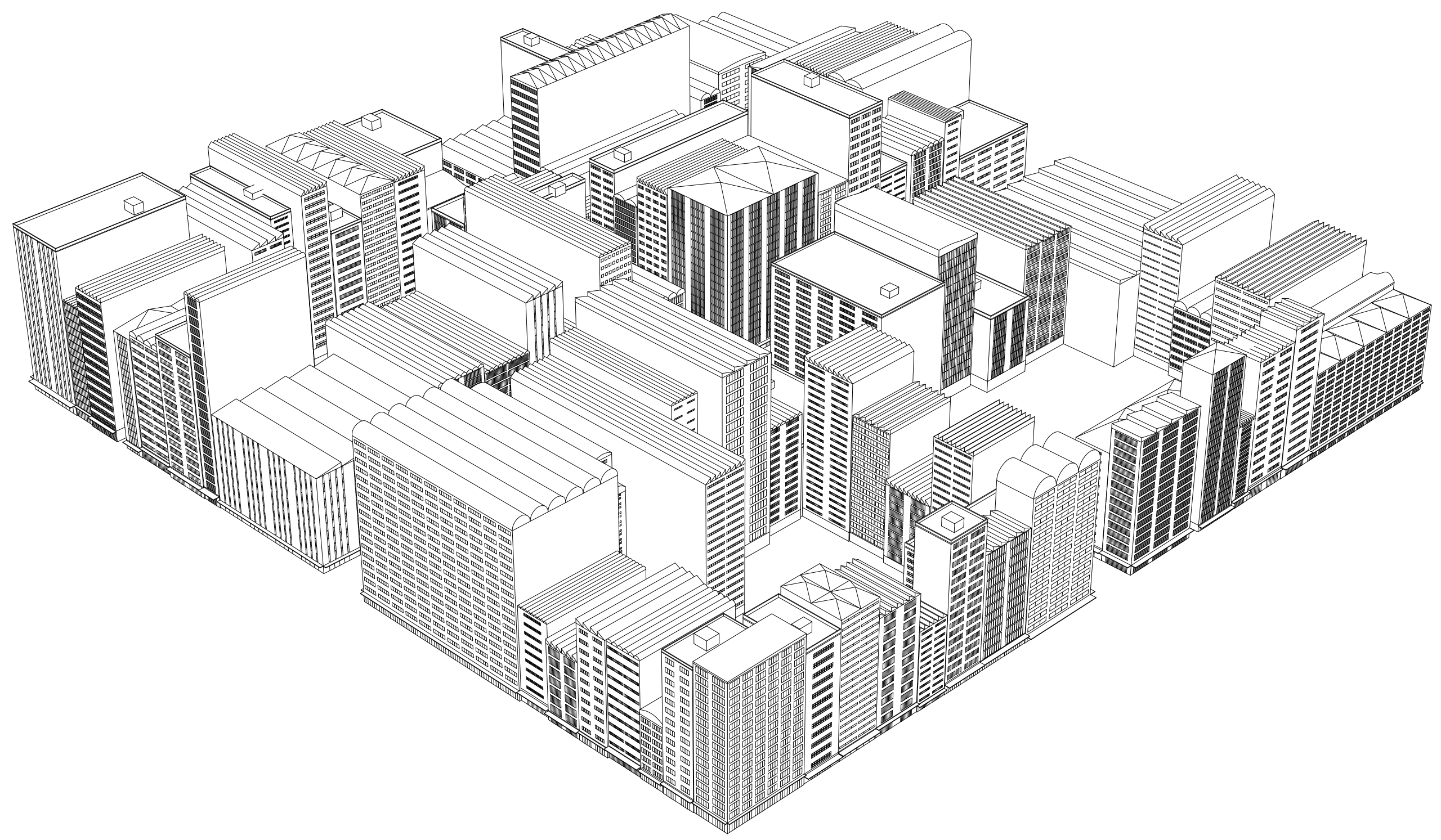

Figure 5.11: Small scale city generator output, 2x2 Blocks, Seed 04 


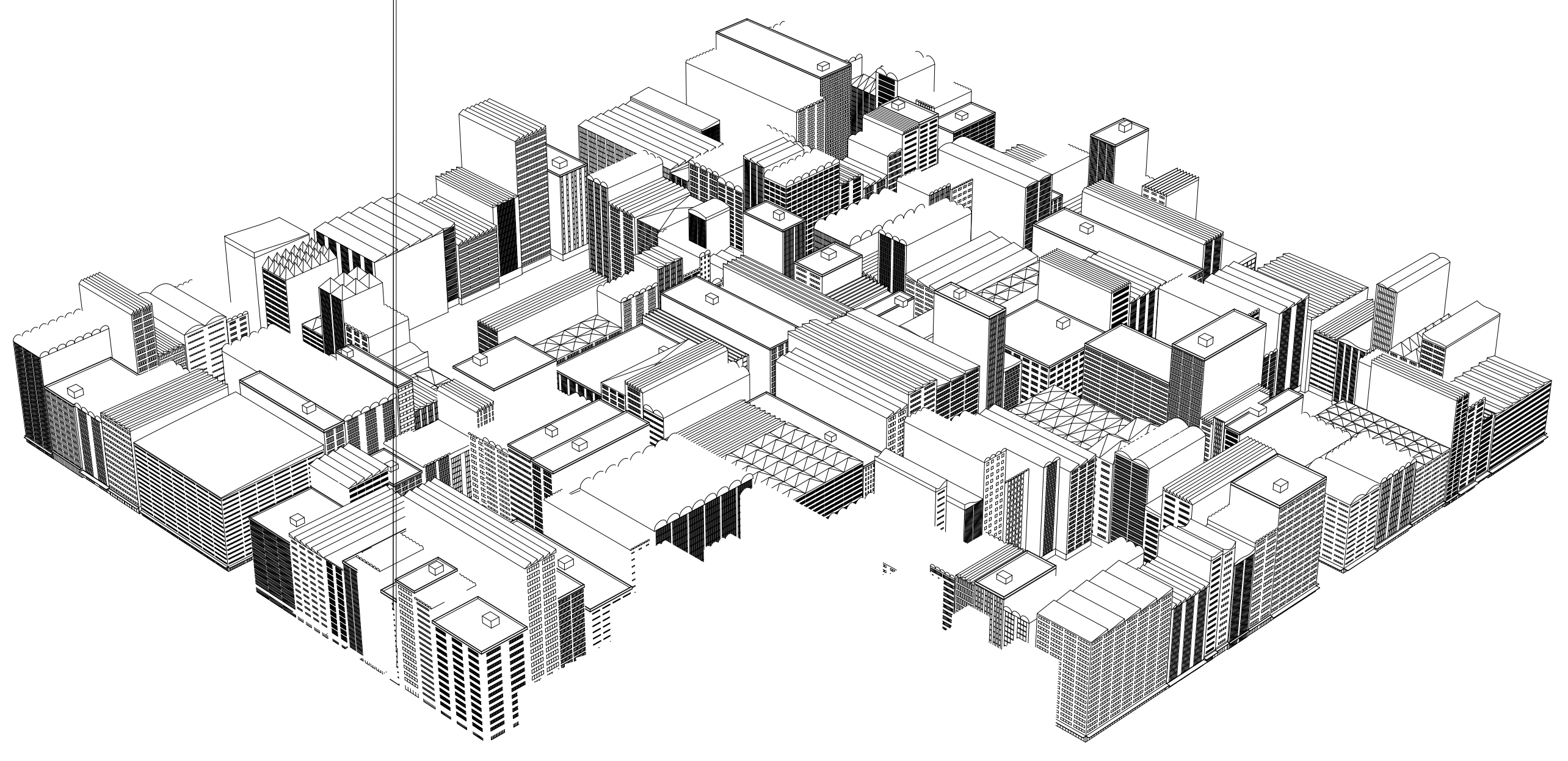




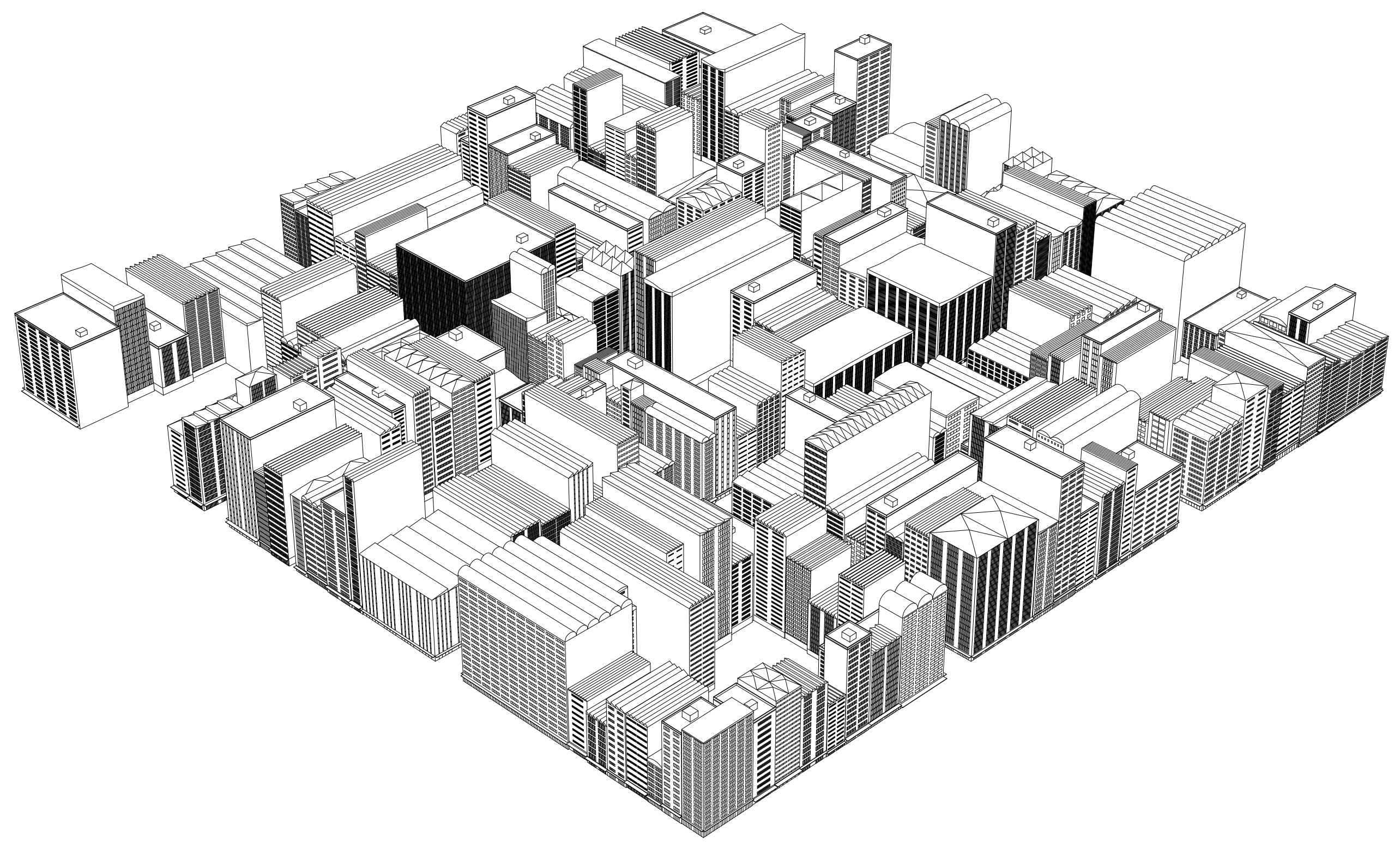

Figure 5. 13: Small scale city generator output, 3x3 Blocks, Seed 04 


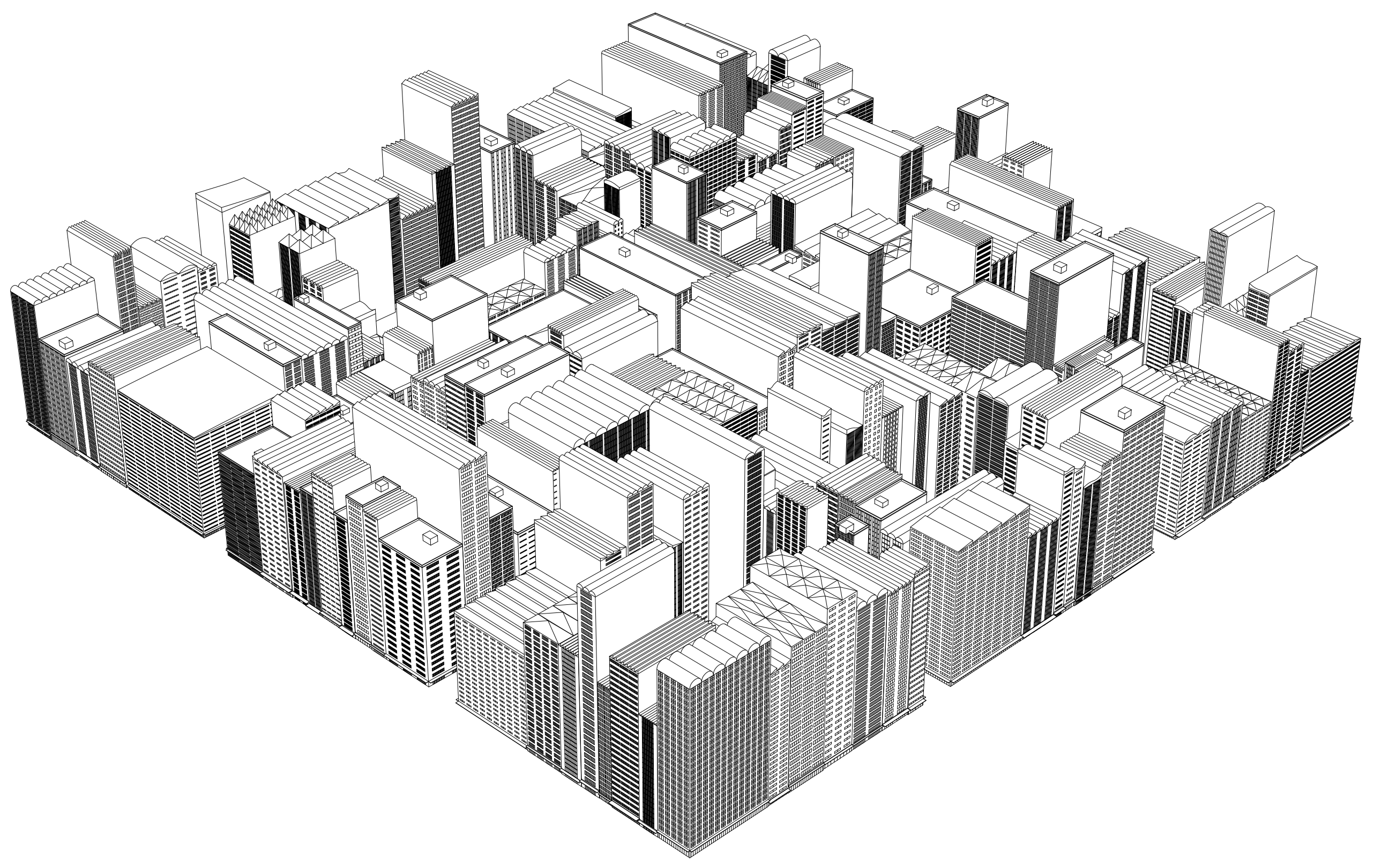

Figure 5.14: Small scale city generator output, 3×3 Blocks, 6-18 Floor Height, Seed 03

CHAPTER FIVE

A CITY IS NOT A TREE 


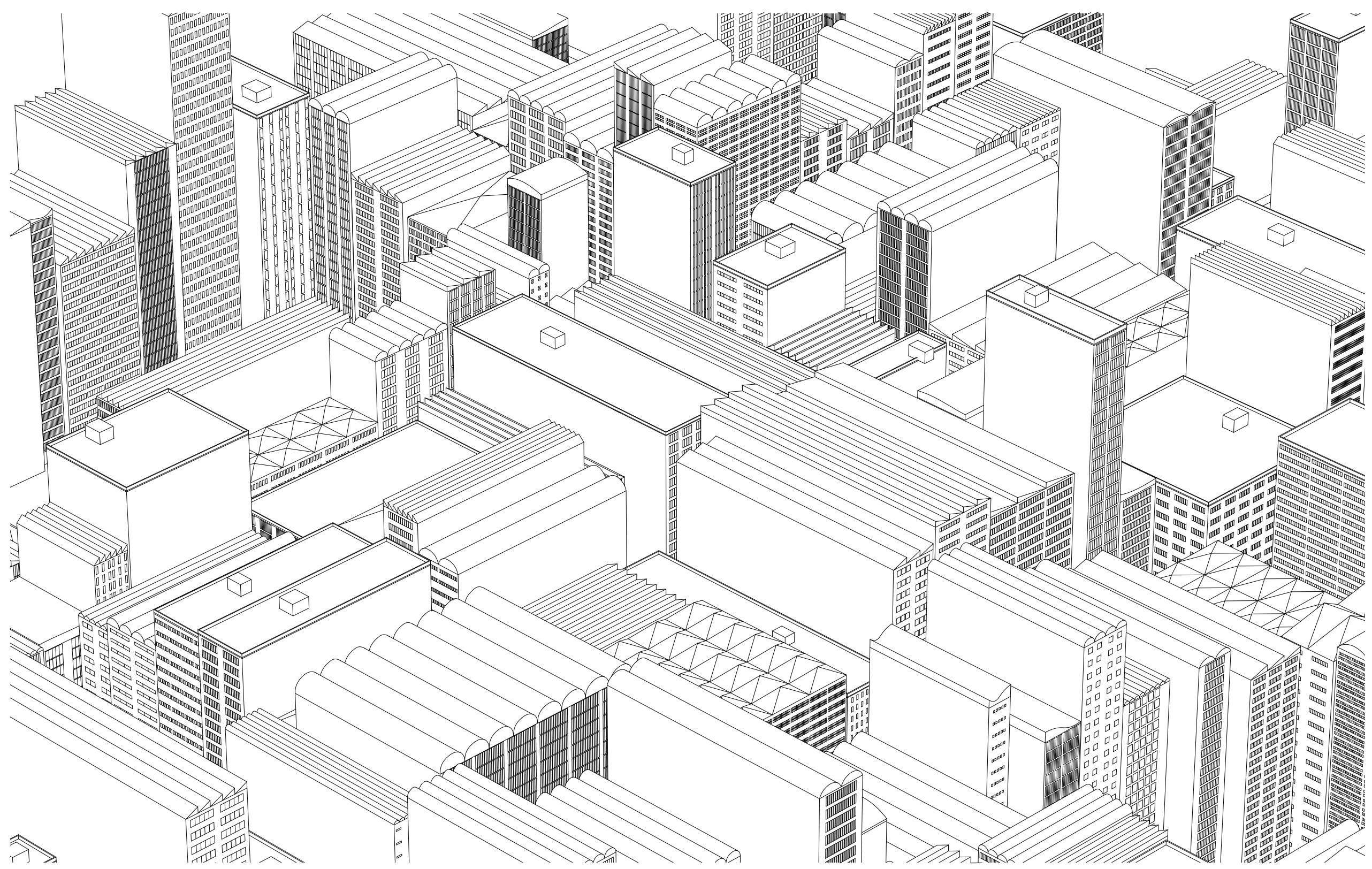

Figure 5.15: Small scale city generator output, 3×3 Blocks, 6-18 Floor Height, 200\% zoom, Seed 03 


\section{HEIGHT AWARENESS}

To build upon the outcomes generated by the small-scale city generator an investigation into the integration of height awareness began. The main inspiration for this inquiry was the blatant lack of any windows on the sides of the buildings. However, it was also clear that generating windows for the full height of each building will be incredibly inefficient, and increasing the processing time significantly.

Through list manipulation within Grasshopper it was possible to organise the building footprints of any procedurally generated block based in a sequence. With this sequence in place the extrusion factor for one building could be measured against the buildings before and after it. As a result of this process, if any of the floors on the building were clear of their neighbour then the wall would be highlighted. This action demonstrated that this wall was a suitable location to generate windows. Further to this, the roof structures understand that they are not a part of the occupiable volume, and as such would not become highlighted.

The highlighted walls have not been given windows in Figure 5.16. However, the logic for installing them is straight forward. This process would involve taking the original list values from the buildings with the highlighted walls and distributing those values as appropriate. The generation of window geometries on these walls could then be controlled by RNG to prevent their appearance in every possible location. 


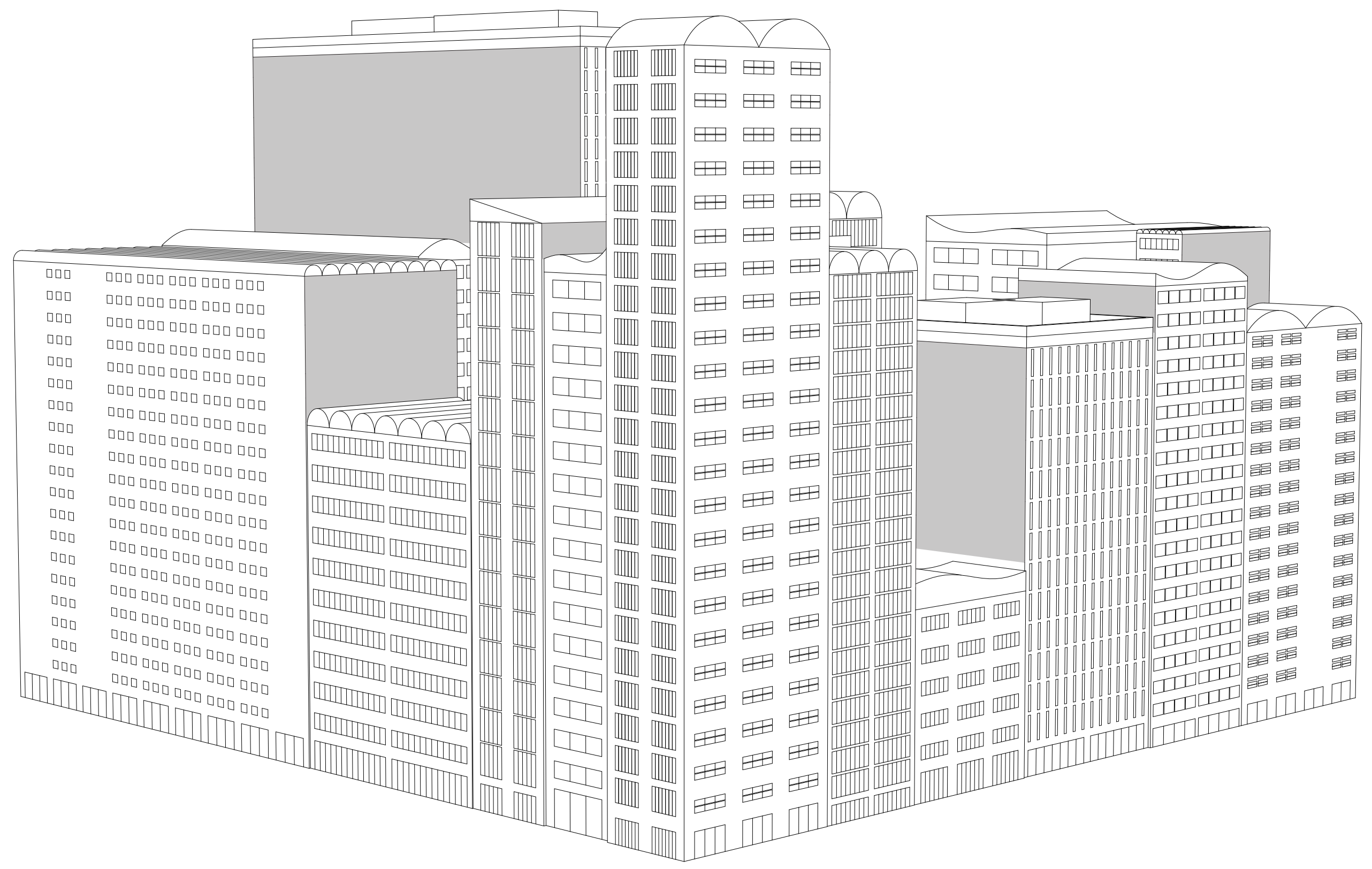

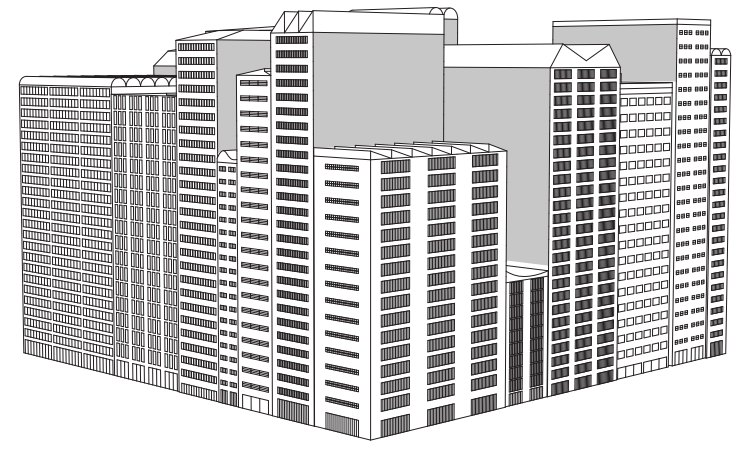

02

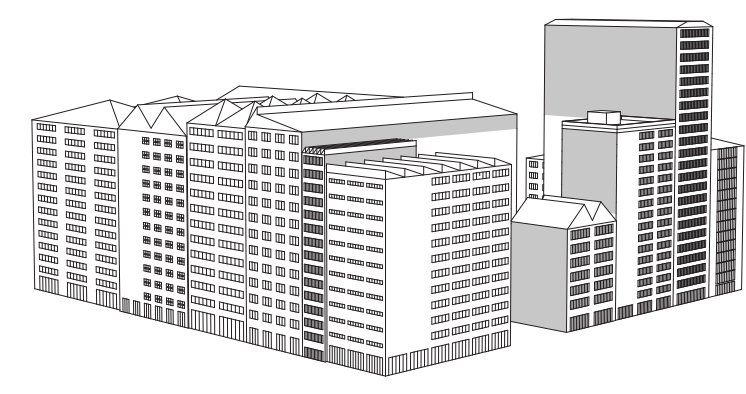

03

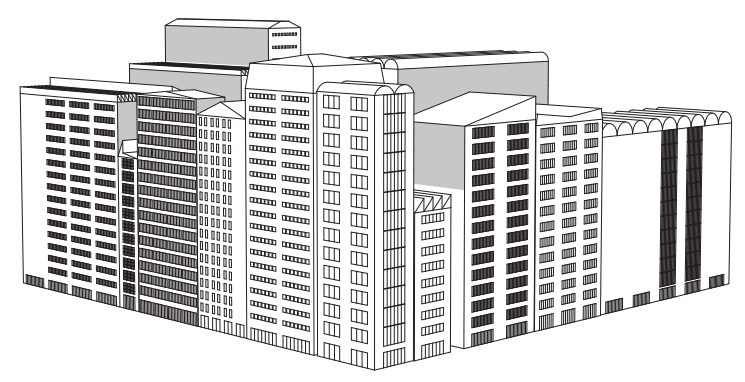

04

Figure 5.16: Compilation of extruded block generator with building height awareness outputs 


\section{OPTIMISATIONS - WINDOWS}

Table 01 demonstrates a significant difference in the time taken to generate three sets of windows. The approach and processes used to create each set of windows are slightly different, but the impact is immediately recognisable in the processing time. While there is a difference in the visually observable information in 02 \& 03 (Fig. 5.17), it is minimal, and it is wasteful to use significantly more processing power to repeat the same information again and again. This demonstration also shows that the process behind 01 (Fig. $5.17)$ is completely unnecessary and wasteful.

Table 1: Windows

\begin{tabular}{|c|c|c|c|}
\hline Geometry & 01 & 02 & 03 \\
\hline $\begin{array}{c}800 \text { Individual Windows (All } \\
\text { independent surfaces) }\end{array}$ & $\begin{array}{c}\text { 80 Surfaces, } 240 \text { lines } \\
\text { added to separate } \\
\text { windows }\end{array}$ & $\begin{array}{c}80 \text { surfaces, text as } \\
\text { necessary } \\
(4 \mathrm{H} / 1 \mathrm{~V}=4 \text { Horizontal, } \\
\text { Vertical) }\end{array}$ \\
\hline Generation Time & $51 \mathrm{~ms}$ & $18.6 \mathrm{~ms}$ & $7 \mathrm{~ms}$ \\
\hline
\end{tabular}

\section{OPTIMISATIONS - FLOORS}

The floors provide another opportunity that is equally simple, with massive opportunities for optimisation. At the current point in time each floor is treated as its own rectangular volume. For individual buildings this isn't a concern, however, when dealing with a number of buildings then these minor inefficiencies become far more prominent. This table demonstrates that the possible savings across the entire system are nothing short of significant.

Both these and other optimisations result in the reduction of clutter within the Grasshopper canvas, and the resultant system as a whole. This makes the system easier to understand, and subsequently more modifiable and reusable.

Table 2: Floors

\begin{tabular}{|c|c|c|c|c|c|}
\hline & 01 & 02 & 03 & 04 & 05 \\
\hline Buildings & \multicolumn{5}{|c|}{25} \\
\hline Floors & 15 & 15 & 0 & 0 & 15 \\
\hline Total Volumes & 375 & 375 & 25 & 25 & 25 \\
\hline Faces & 6 per & 4 per & 6 per & 6 per & 6 per \\
\hline Total Faces & 2250 & 1550 & 150 & 150 & 150 \\
\hline Additional & 25 caps (1 for & & $\begin{array}{c}\text { Text tags (For } \\
\text { floor heights) }\end{array}$ & $\begin{array}{c}375 \text { rectangles } \\
\text { (For visual } \\
\text { clarity) }\end{array}$ \\
\hline $\begin{array}{c}\text { Generation } \\
\text { Time }\end{array}$ & $96 \mathrm{~ms}$ & $88 \mathrm{mms}$ & $8 \mathrm{~ms}$ & $16 \mathrm{~ms}$ & $16 \mathrm{~ms}$ \\
\hline
\end{tabular}




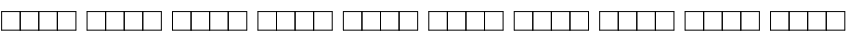

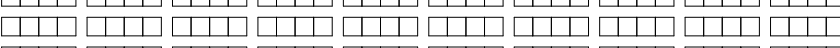

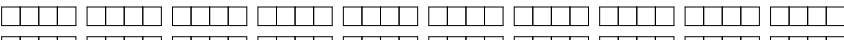

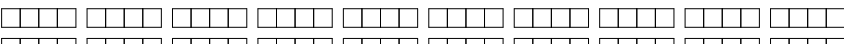

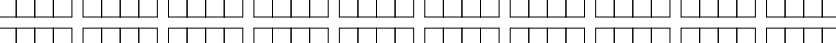

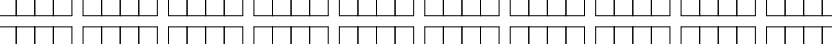

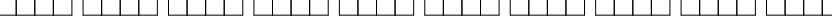

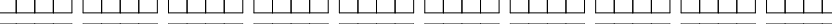
(1) (1) पात (1)

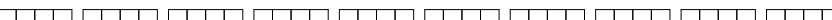

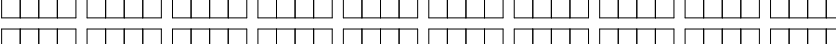

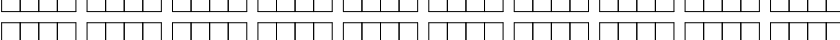

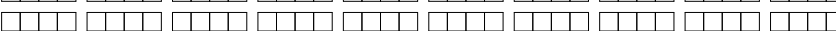

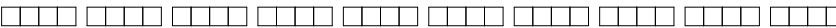

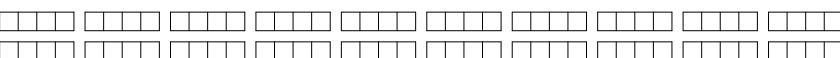
म

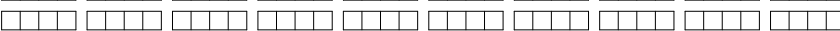

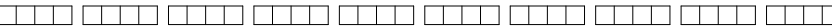

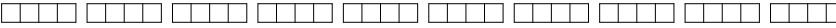

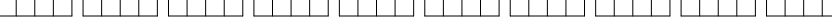

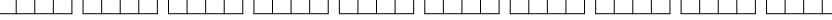

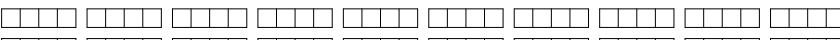

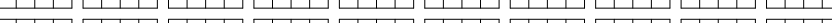

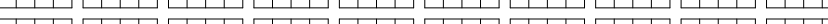
$\square \square \square \square \square$

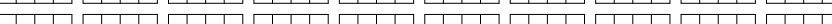
का

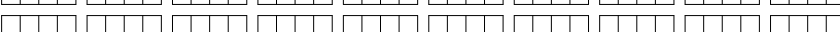

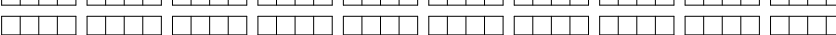

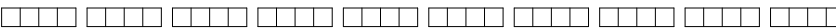

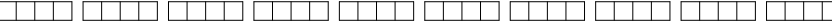

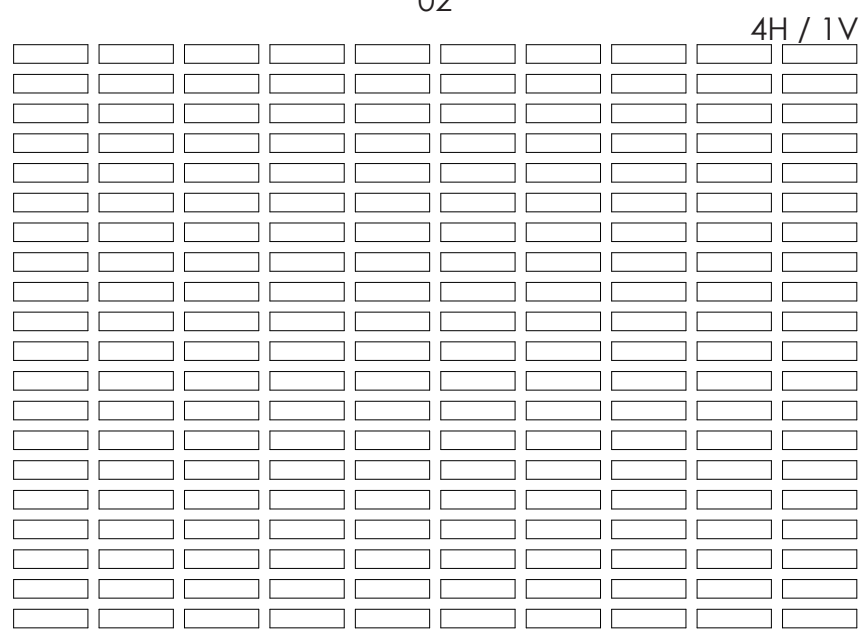

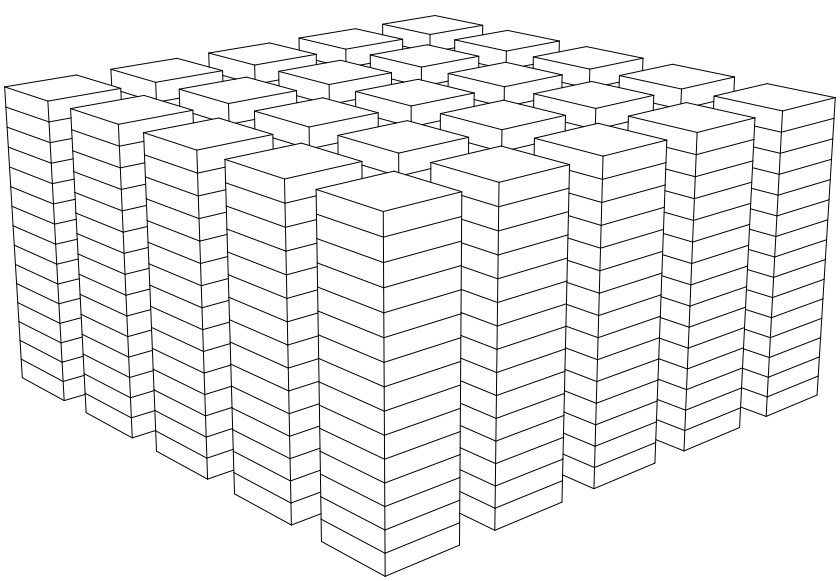

$01 / 02$

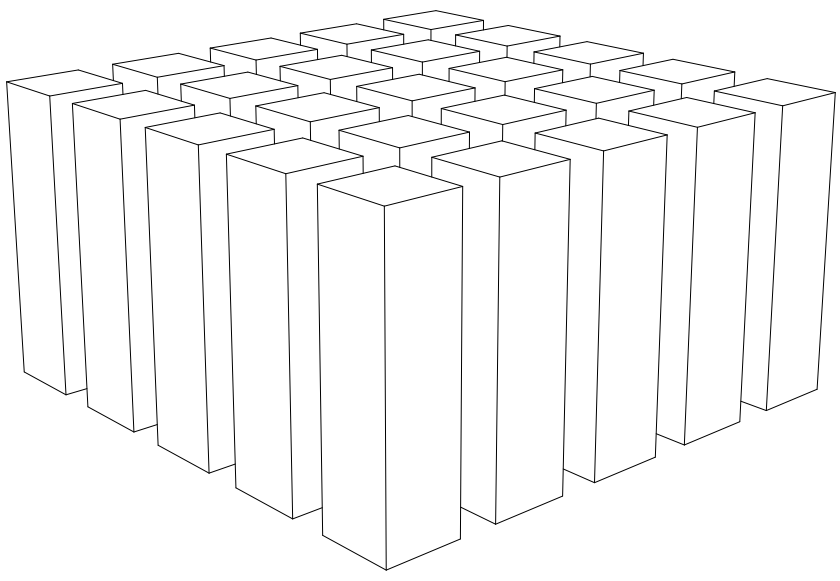

$03 / 04$

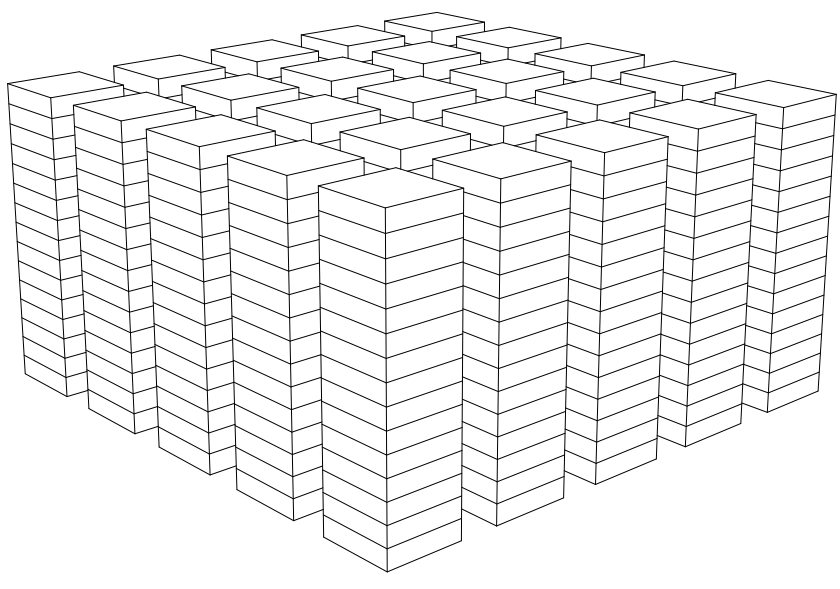

05 


\section{OPTIMISATIONS - FOOTPRINT GENERATOR}

Further optimisation can occur in the reduction of the overall number of components, an area rife with optimisation opportunities. Optimisation in this area reduces processing times and significantly improves the manageability of any given document.

One area where this is relevant is in the creation of building footprints. In all prior iterations, the city generator has formed individual building footprints based on point divisions, minimum and maximum values, and scaling factor. Iteration's One through Four demonstrate this in their block generators.

Any reduction of components in this area has massive flow-on effects relating primarily to efficiency, and data management. When working in Grasshopper actions such as these are often grouped into clusters.

While separating these clusters and treating them individually is tempting, it is far easier to modify the data at a later stage if each cluster remains interconnected. However, this requires a significant amount of planning to function as intended, including consideration of essential elements such as directionality. By linking these elements to logic gates the correct data is distributed each and every time. This action allows any clusters to remain interconnected and retain a high level of modifiability.

Figures 5.20 and 5.21, demonstrate a more optimised footprint generator than has been shown in previous iterations. Improvements in these outcomes are noticeable in regards to speed, adjustability, and user control. The key difference in this iteration is a shift from point based line division to scaling values. Where the original could only create as many building footprints as there were components this version is easily modifiable to generate any number of footprints, in a far more efficient and reliable manner. This is a situation where achieving the desired outcomes takes significantly longer than setting it up in a way that just works, but the reward is immense and has a noticeable impact on the operation of the system.

Facilitating integration with other plugins such as Human.UI (Figure 5.19) also make this information more accessible. Through its use each of the relevant controls are all located in one space. This means that the user is not required to hunt through the Grasshopper canvas looking for tiny sliders and wireless connections. Human.UI makes it incredibly easy to explore a range of highlevel 'design options' through the manipulation of number slider, and makes the system truly accessible (Wonka, 2003).
BLOCK CONTROLLER $-\square \times$

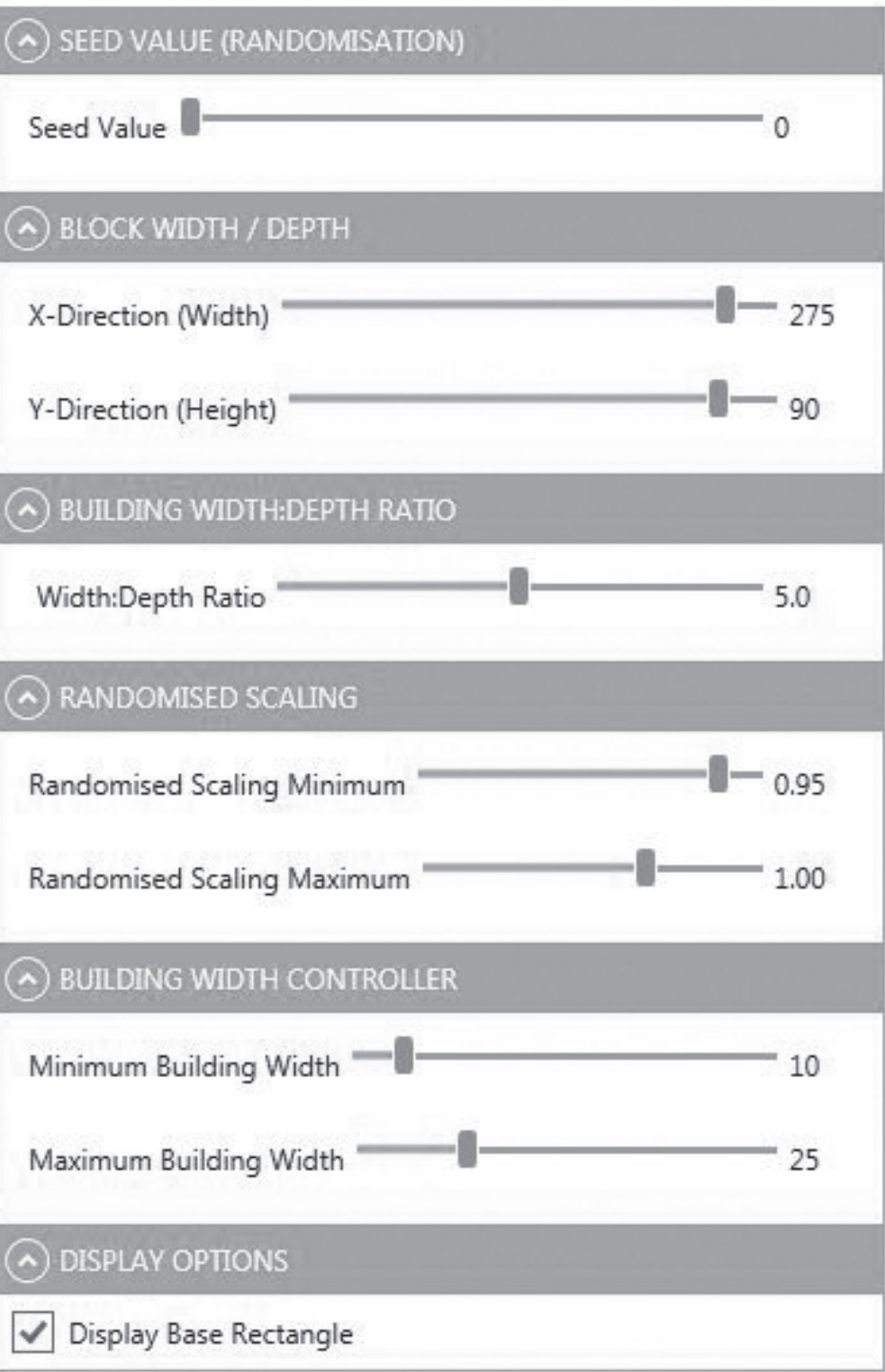

Figure 5.19: Screenshot of Human.UI interface, within Grasshopper 
2.0 Max Width: depth
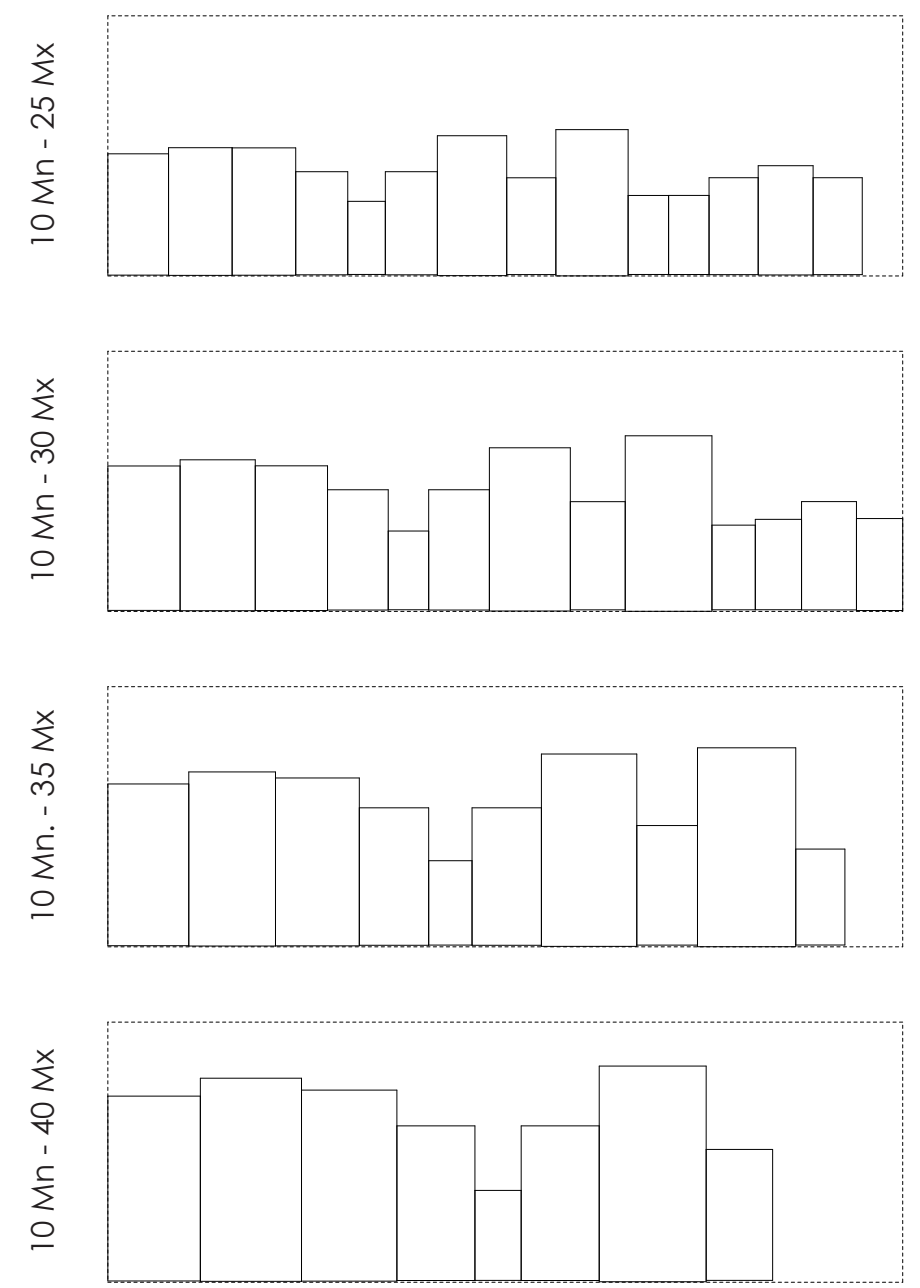

5.0 Max Width: depth
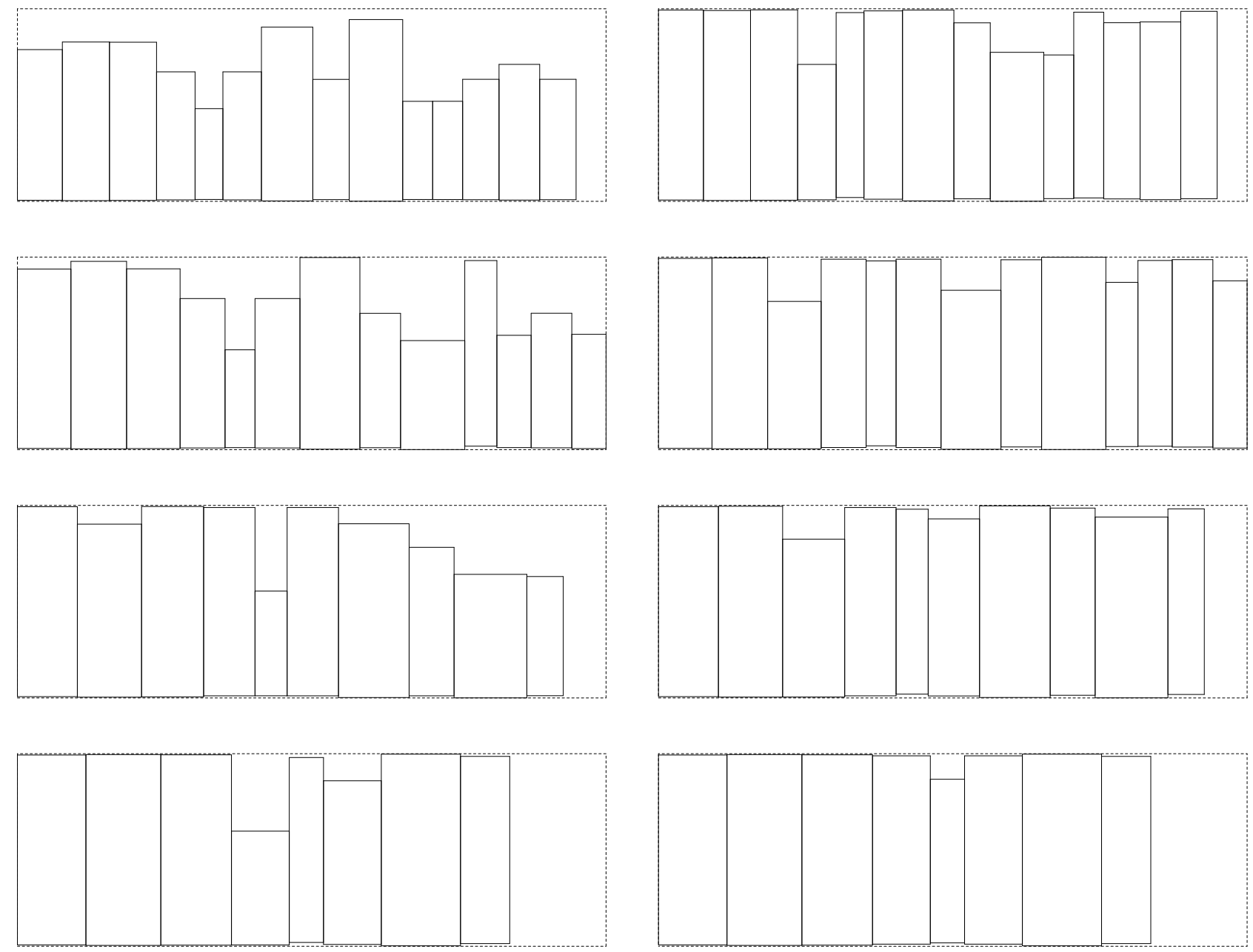

Figure 5.20: Compilation of footprint generator outputs 2.0 to 5.0 maximum width/depth scaling
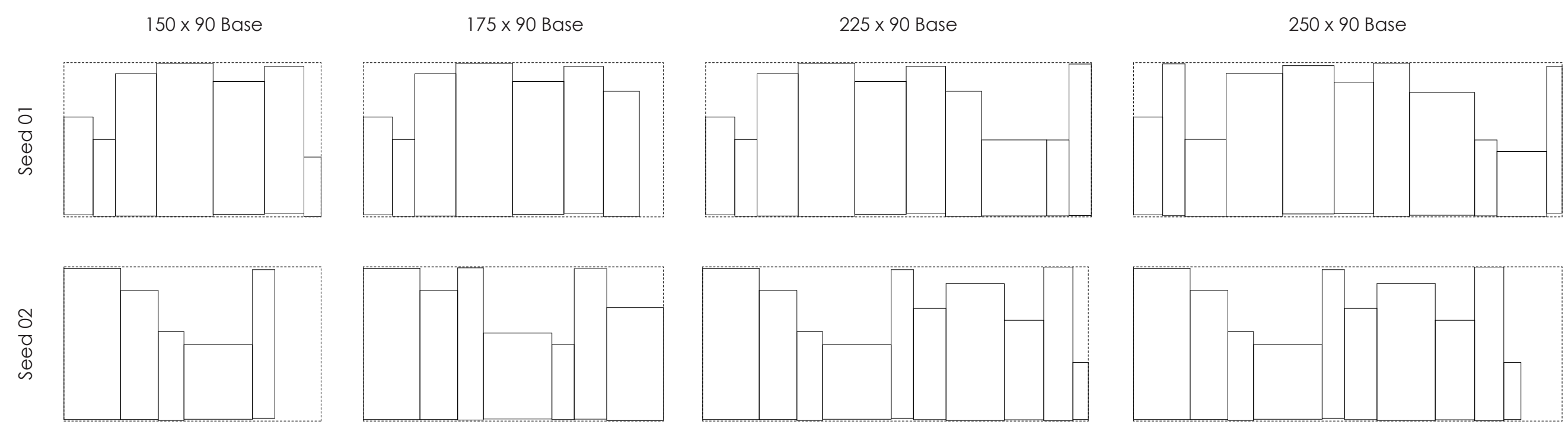

Figure 5.21: Compilation of footprint generator outputs. Same seed on different bases PAGE 102 


\section{CHAPTER SIX}




\section{CREATING THE CITY}

The city scale generator builds upon the work established in early city generation systems, as well as the footprint articulators of displayed as optimisations.

The steps follow an incredibly similar process to the smaller scale developments. The difference with this iteration is that its final unmodified output would be 81 blocks, each with uniquely sized footprints, extrusion, and exterior elements. However, the generator will frequently produce more than 81 blocks. This is because the system measures the area of each generated block against a user-specified range. If the area falls within that range then there is a user specified chance that the block divides into two smaller blocks. If the area is above the maximum specified value then it will automatically be split, as it is considered excessively large. All blocks are then offset internally to create a road network around each.

Once these blocks have are individually defined then the definition from Iteration Four reintroduced. This brings in the earlier idea of building a system that is fit for installation at later stages. With only minor modifications this system is applied to all of the generated block, rather than merely a single block. As with the earlier display of Iteration Four, each building footprint is sized based on a minimum and maximum building width range, a maximum width: depth range, and so on.

In its current iteration, the system will produce 81 unique blocks as a minimum and can produce up to 100. However, this could be modified to allow for the inclusion of any possible number of blocks. The issue with increasing the scale becomes increased processing times, and a greater requirement for tidy data management.

Further articulation of the city scale environment involves the addition of green space and parking areas. These are standard features of any given city, but their inclusion in this system is relatively rudimentary in that they do not have the same degree of articulation as block outputs. Both of these elements are generated based on user specified values. These values have an impact on the generation of elements at each scale, including entire blocks, to segments of the block, and individual footprints. These can be easily modified to create an appropriate amount of green space in the generated environment. While this does not necessarily improve the architectural qualities of the city generator, it adds an important feature that would otherwise be noticeably lacking. 


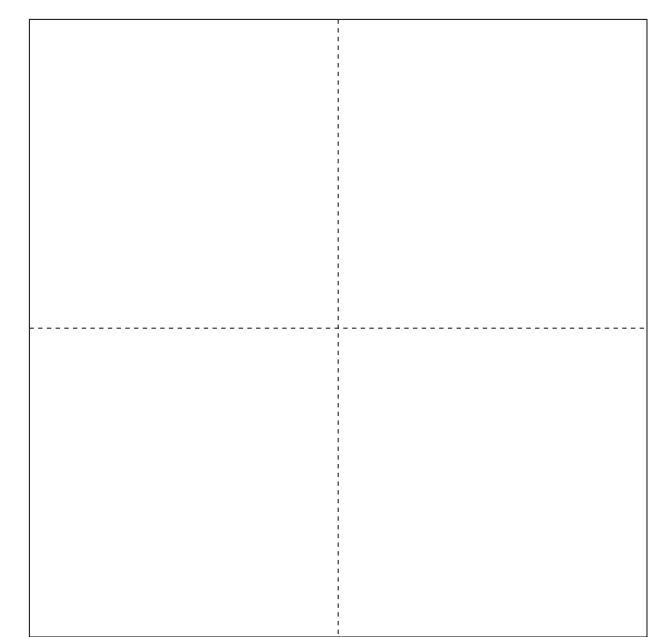

Step 01

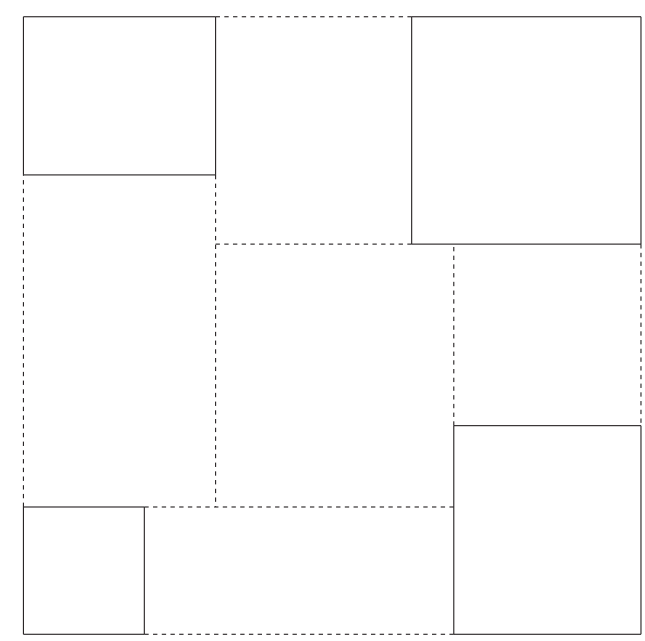

04

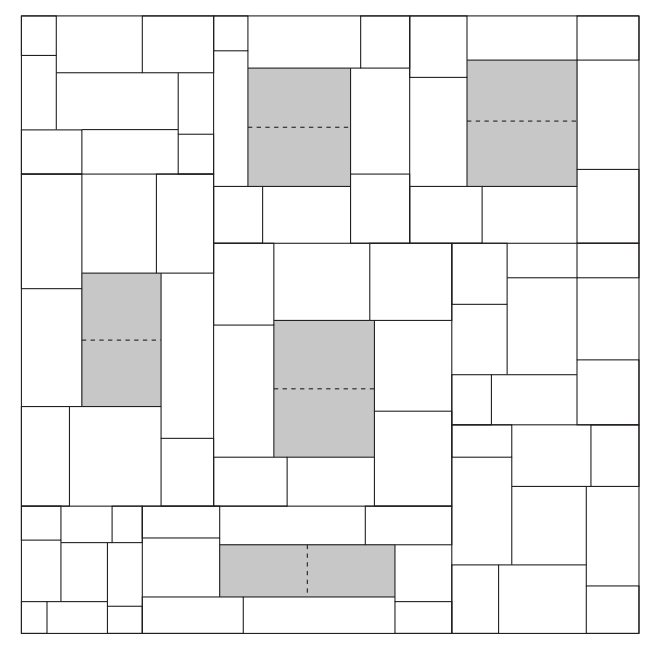

07

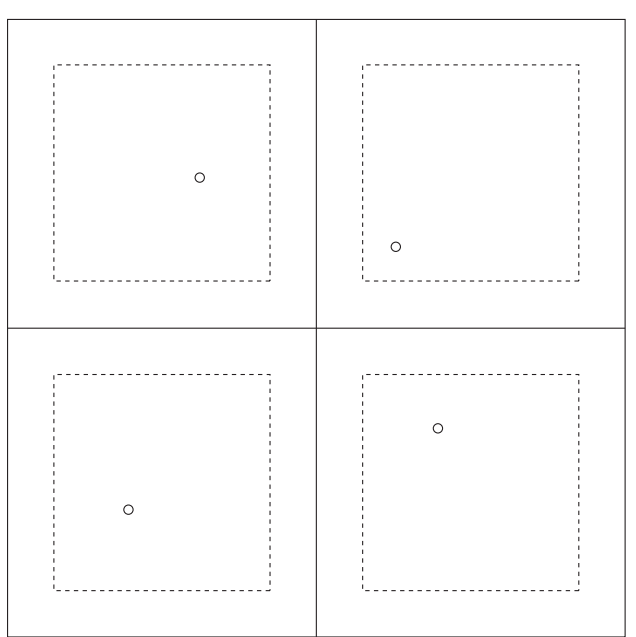

02

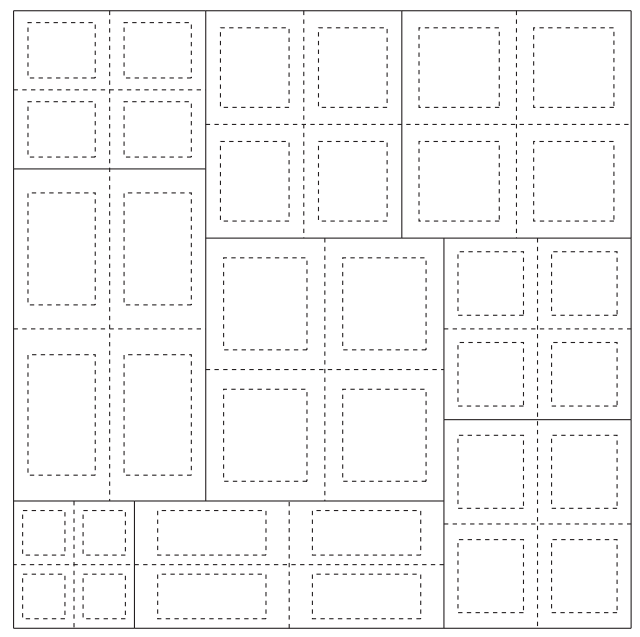

05

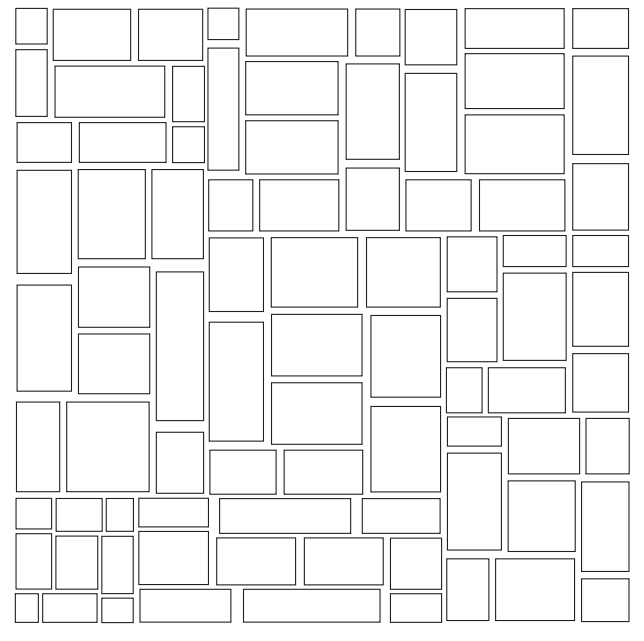

08

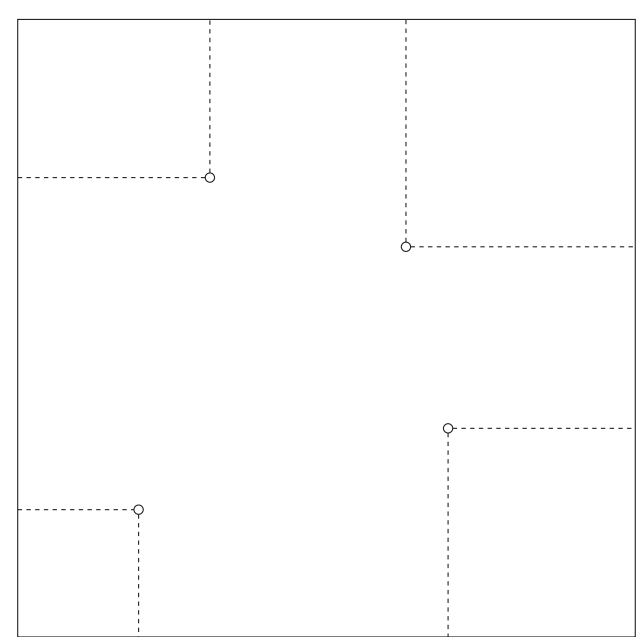

03

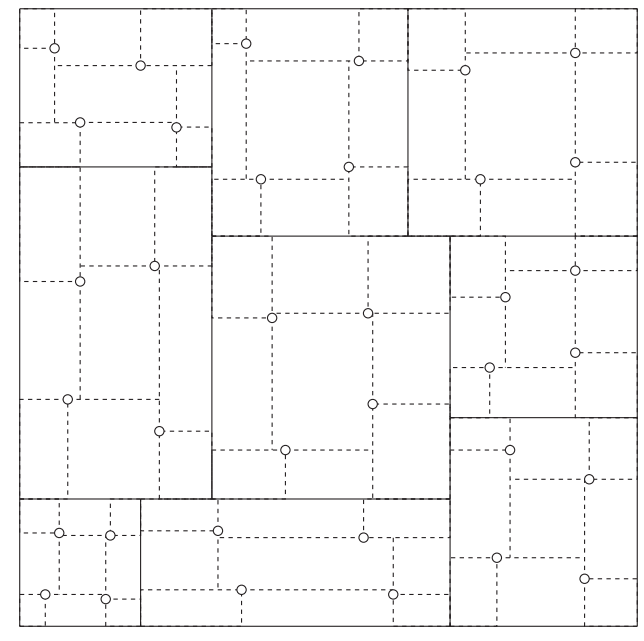

06

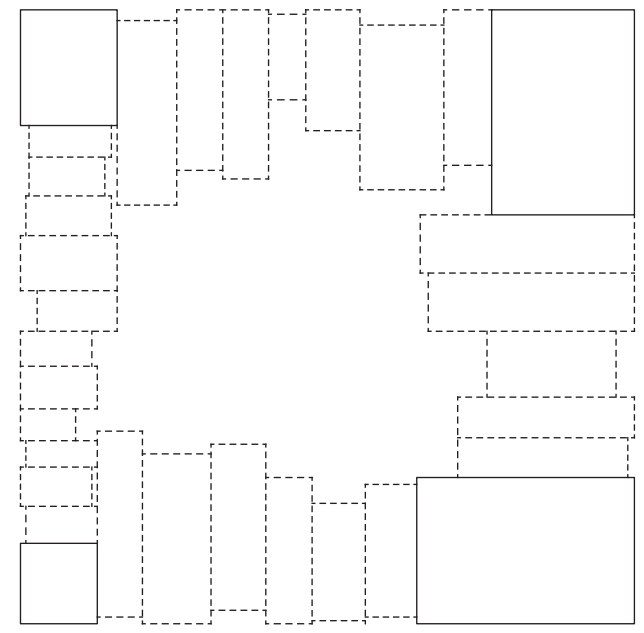

Iteration Four articulation (Every block)

Figure 6.01: Construction of city generator system

CHAPTER SIX

A CITY IS NOT A TREE 


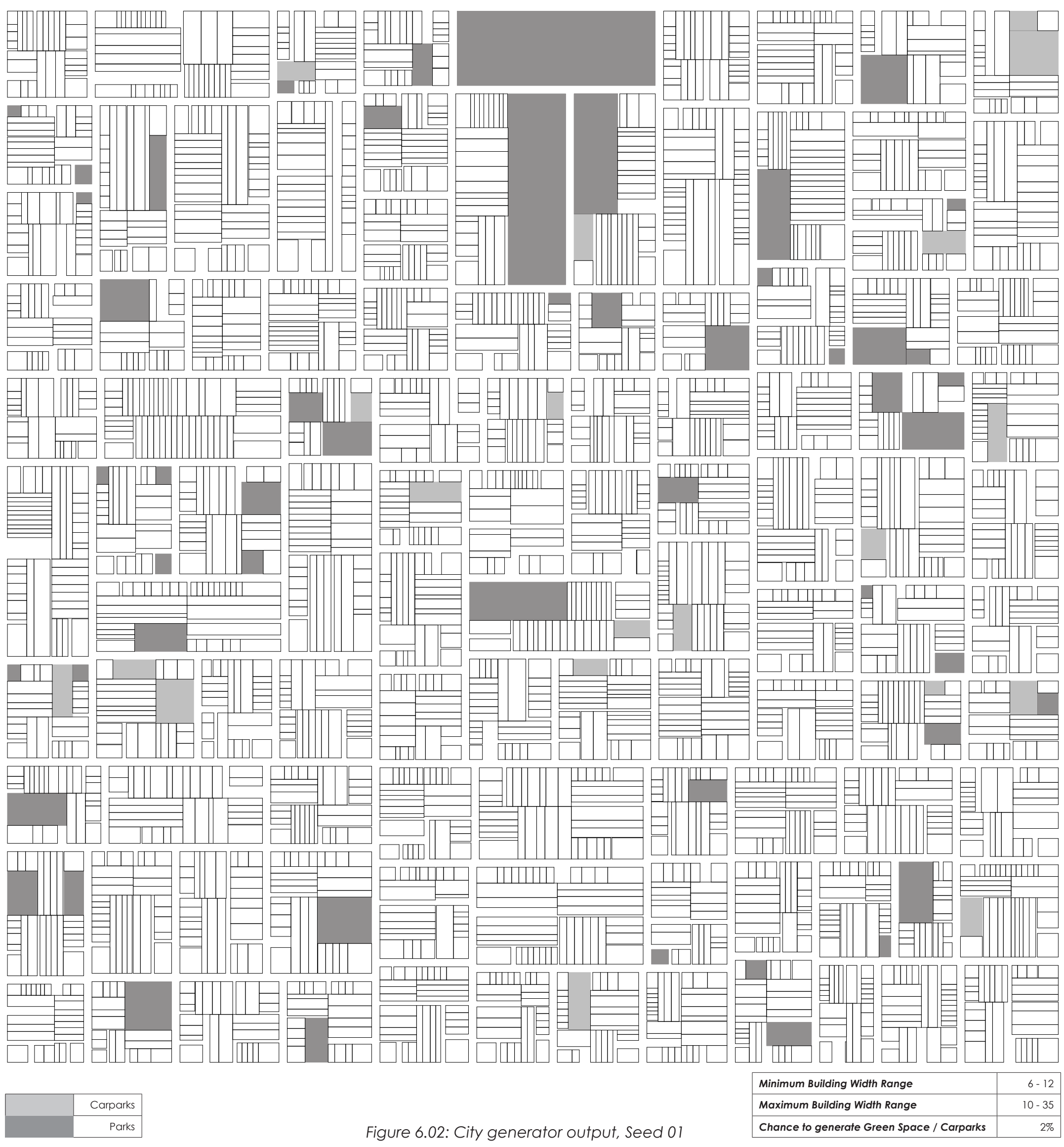


凹ய山 則缃

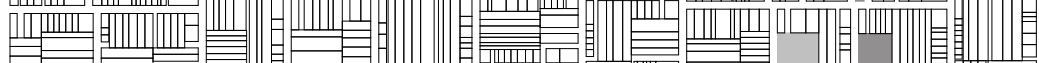
唁 $=$ IIIII

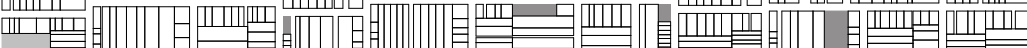
五

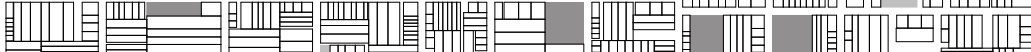
言

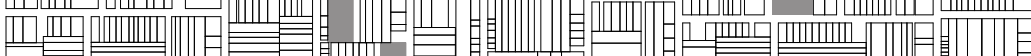

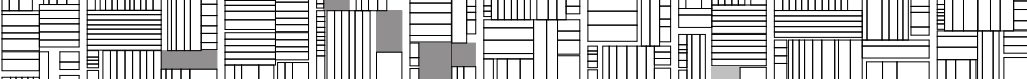

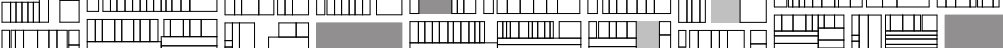

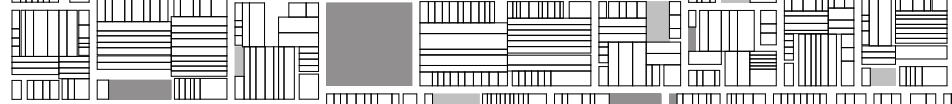

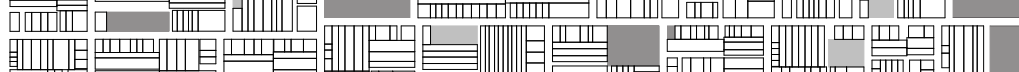
एul| cumm amm

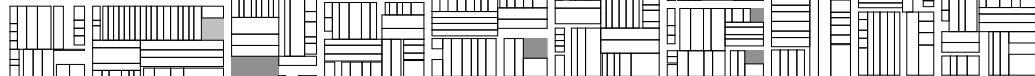
前

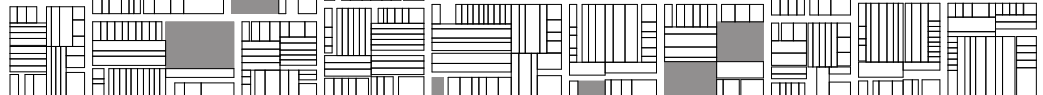
Seed 02

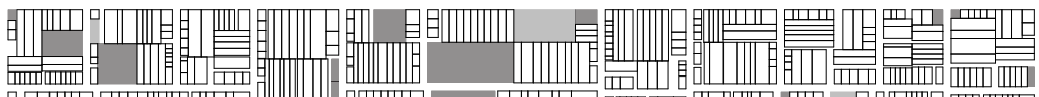

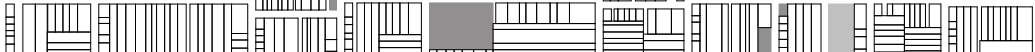
睡 (I||

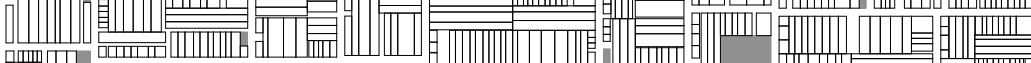

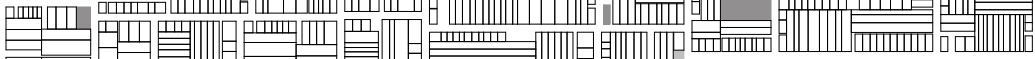

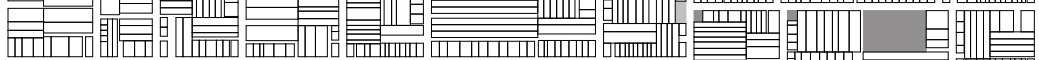

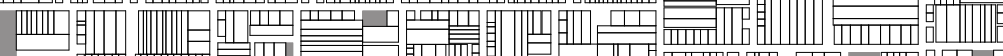

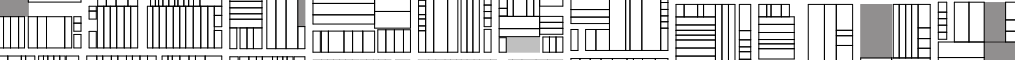
एum| E 目 罗

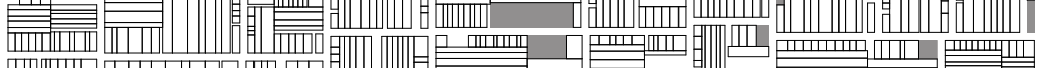

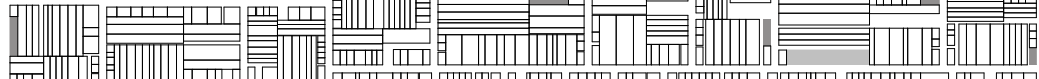

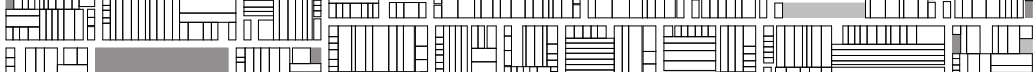

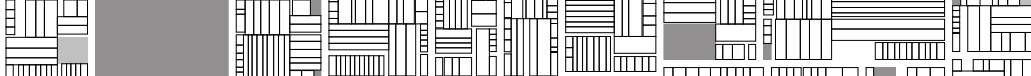

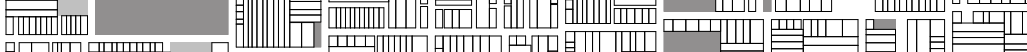

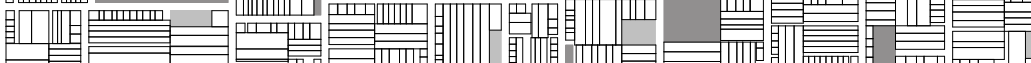
ou

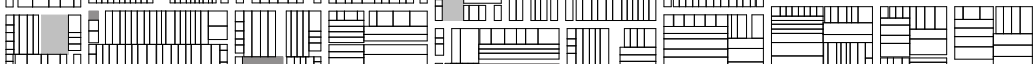

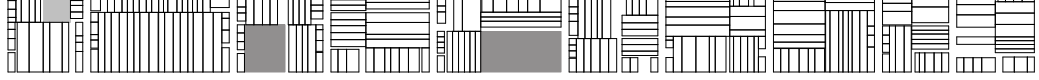

04

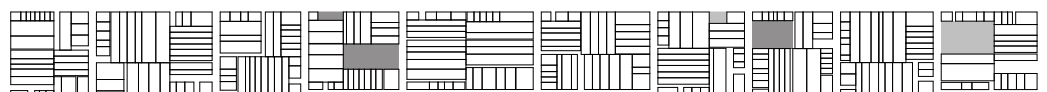

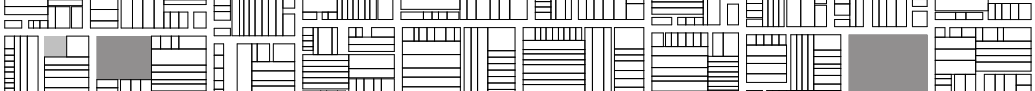
川

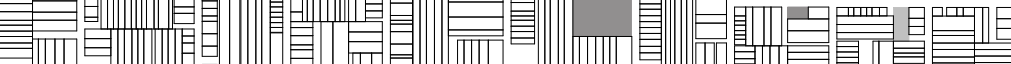
高Un 㑭

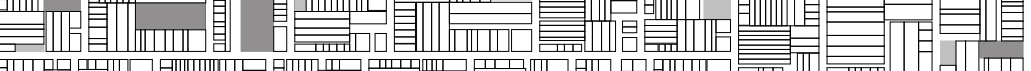

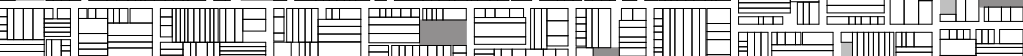

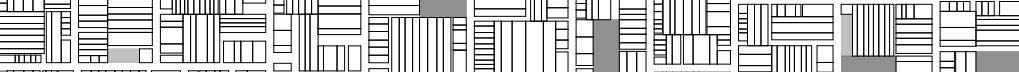

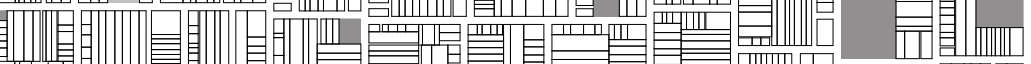

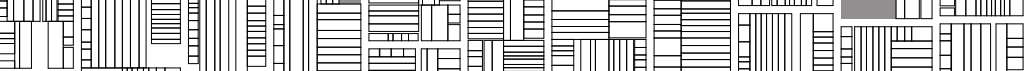

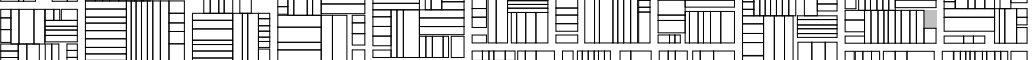

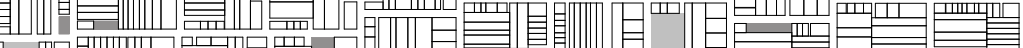

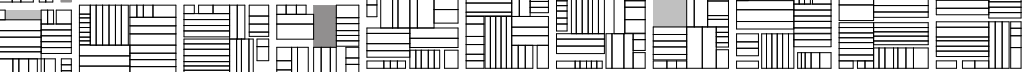
Gll|

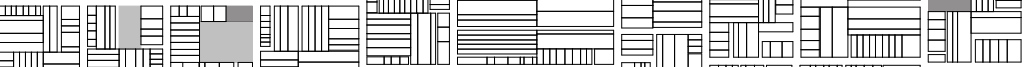

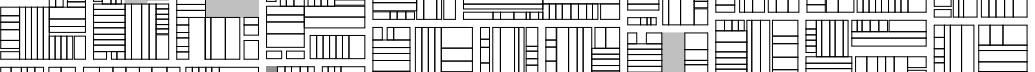

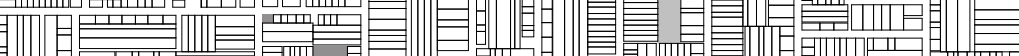
眨

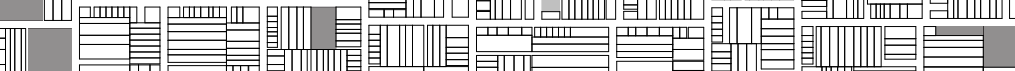

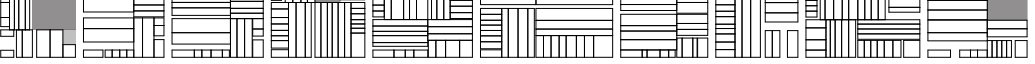
03

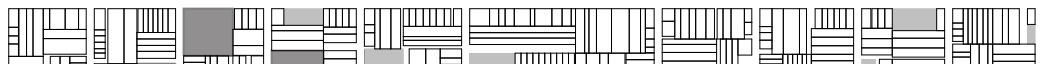

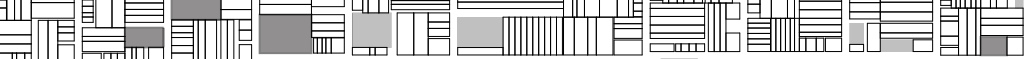

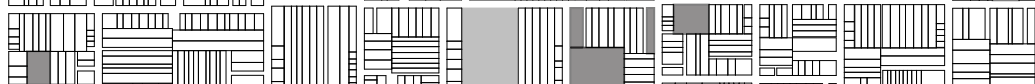

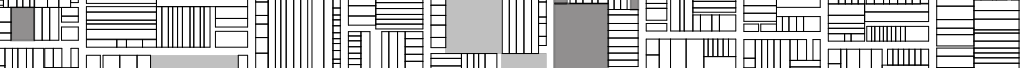

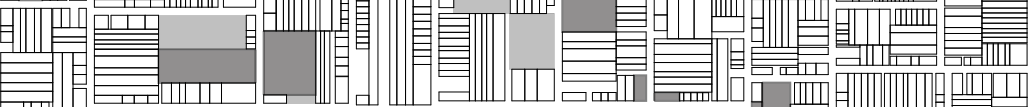

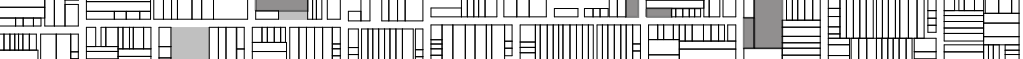

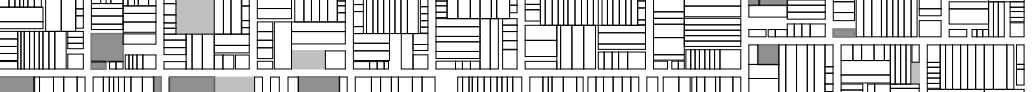

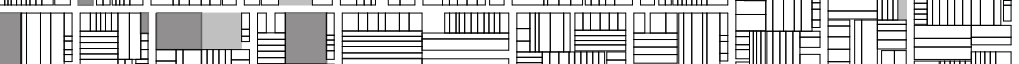
an

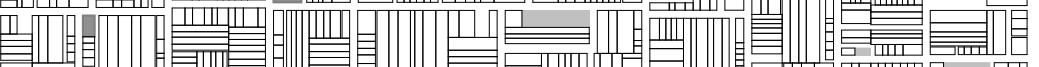

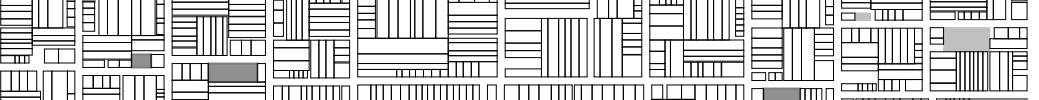
Im

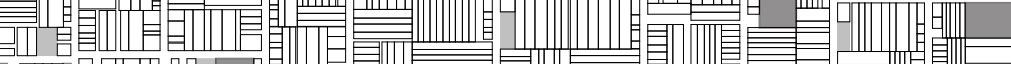
口菂

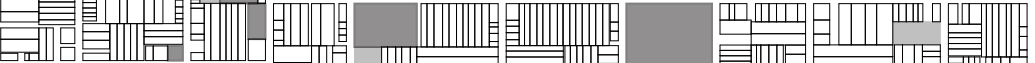

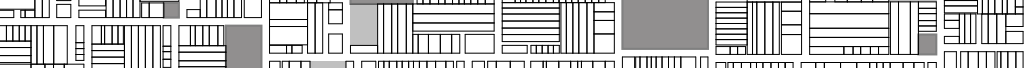

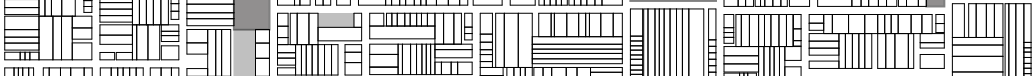
हm

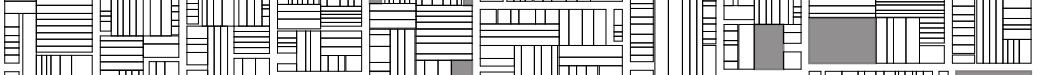

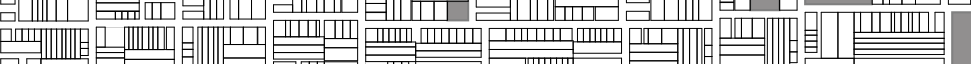

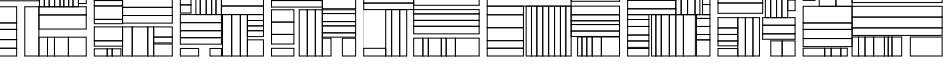

05

Figure 6.03: Compilation of city generator outputs, lowest possible park/carpark generation

CHAPTER SIX 


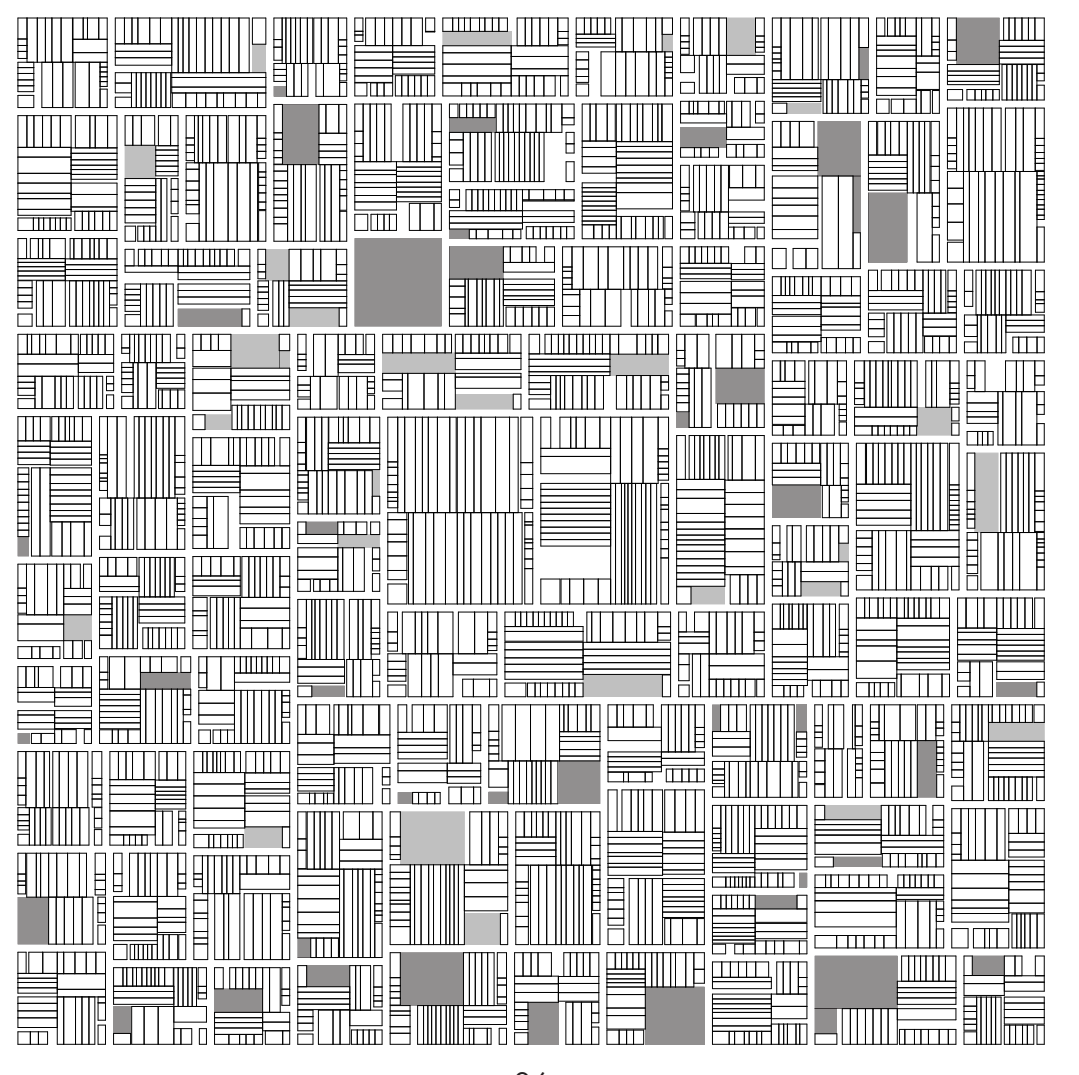

06

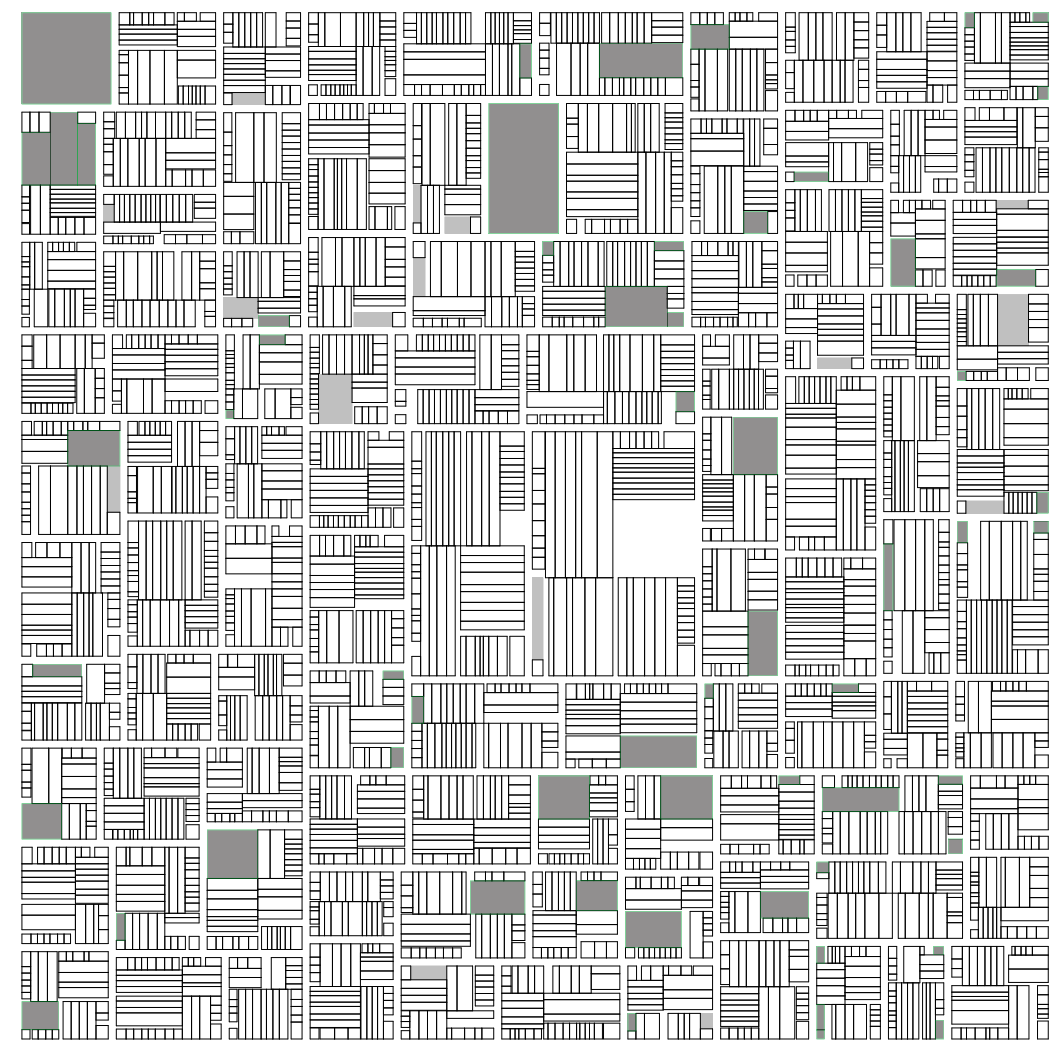

自

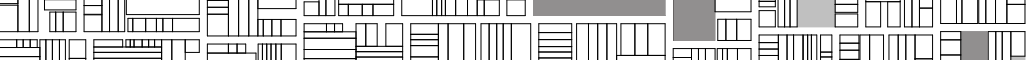

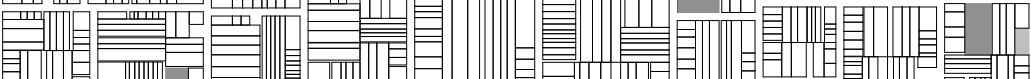
貝

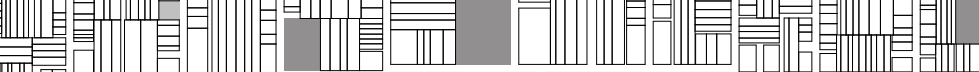
III

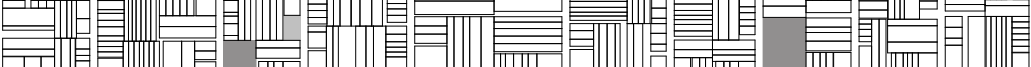

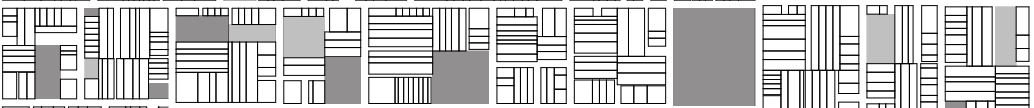

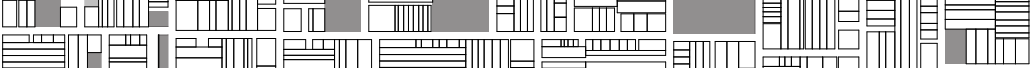

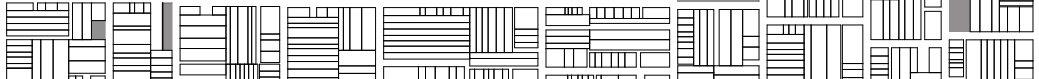

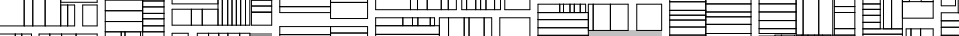

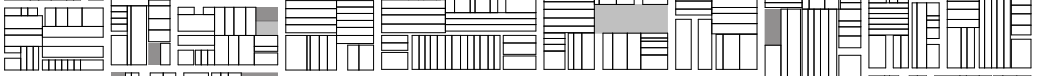
穴

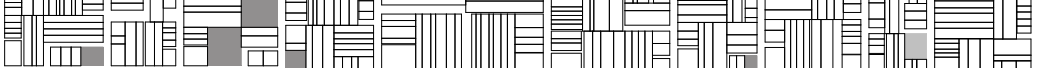

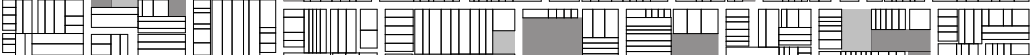
U 目 " =

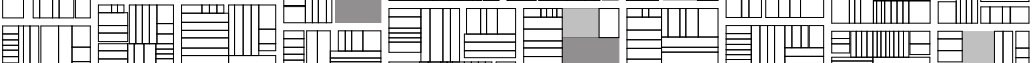

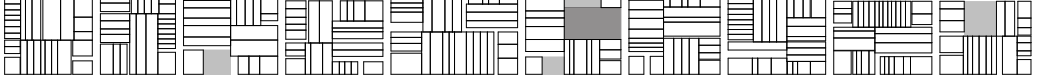
07

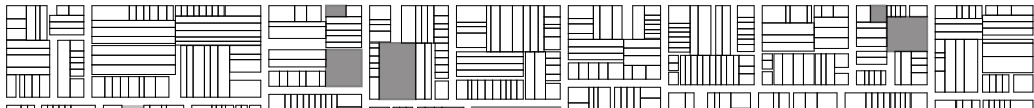

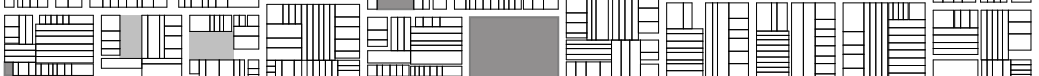

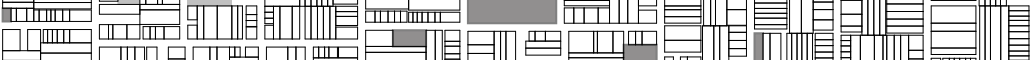

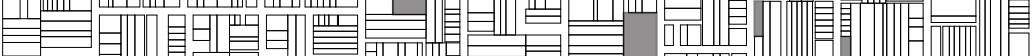

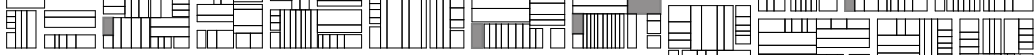

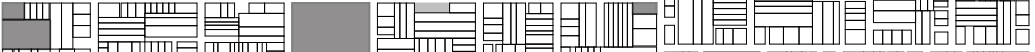

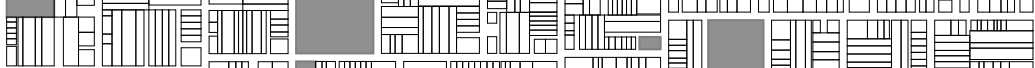
प10m

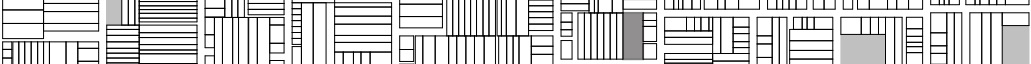
地

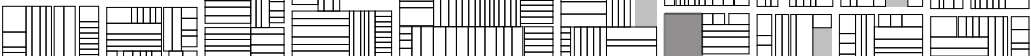
准

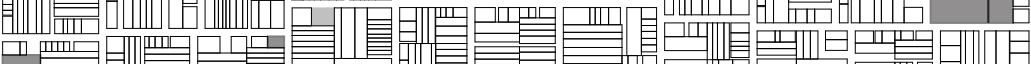

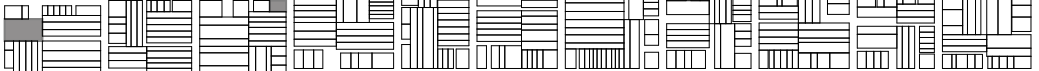

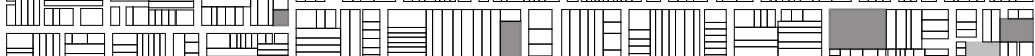

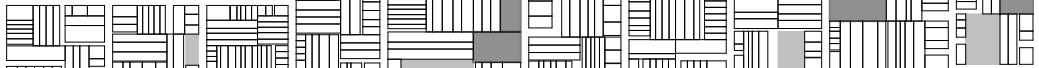
एسm

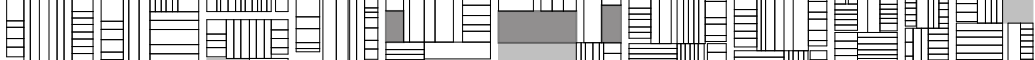

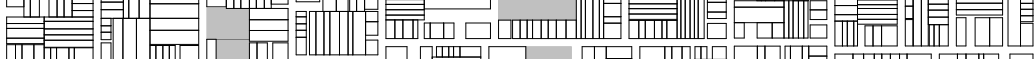

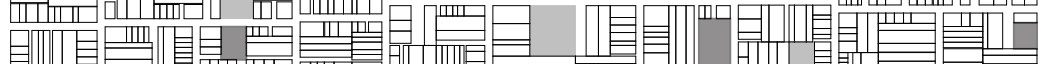

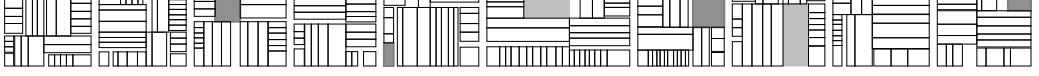
09

Figure 6.03: Compilation of city generator outputs, lowest possible park/carpark generation (cont.) 


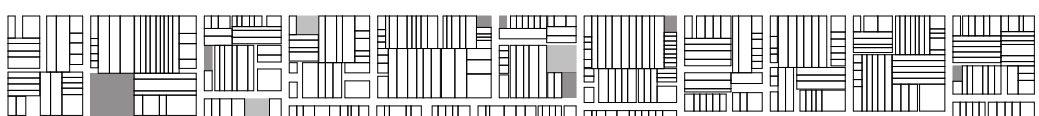

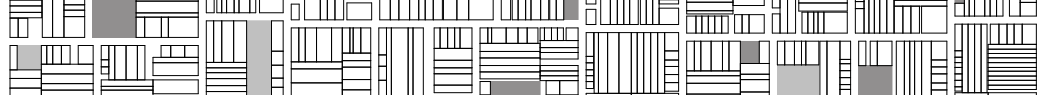

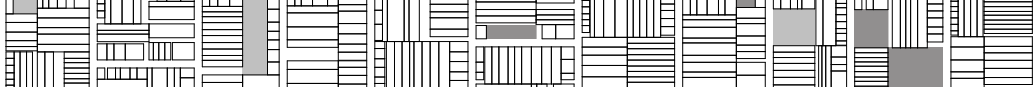

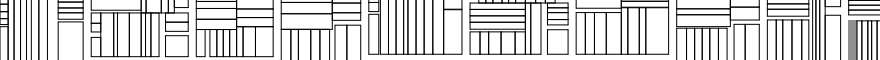

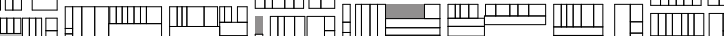
至 and

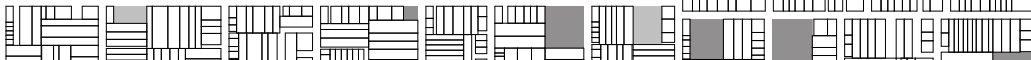

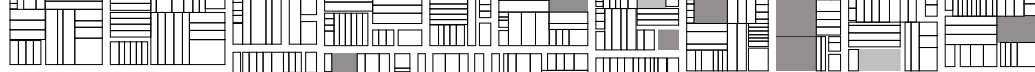

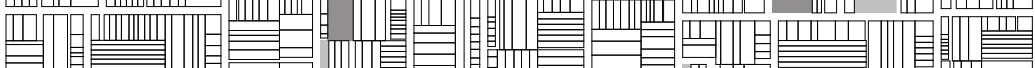

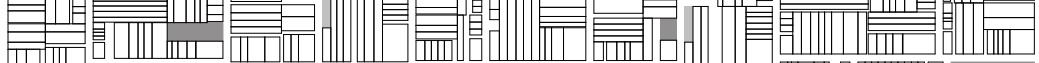

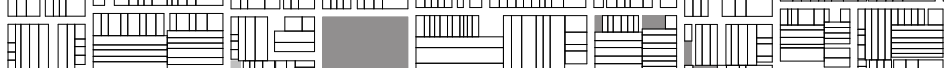

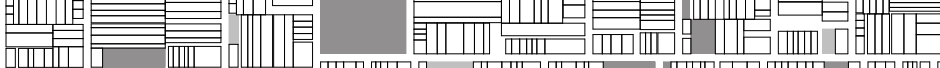

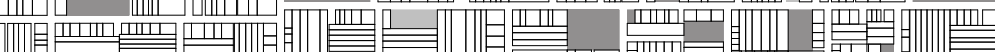

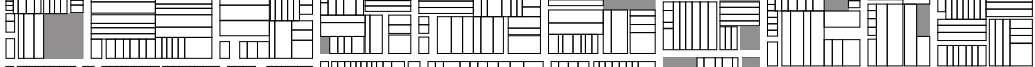
口um 口um

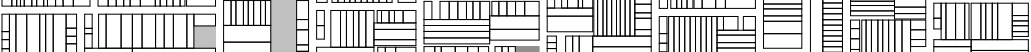

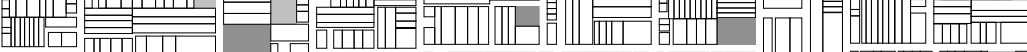
me

Seed 02 - Park \& Carpark Generation Chance (PCGC) 3\%

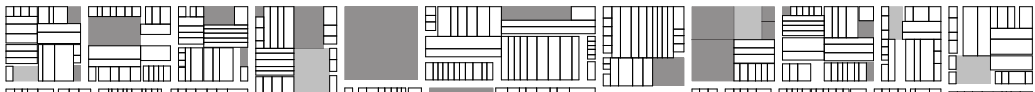

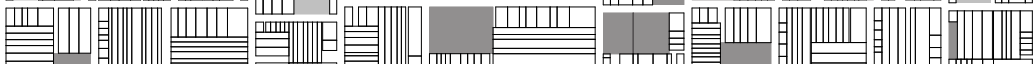

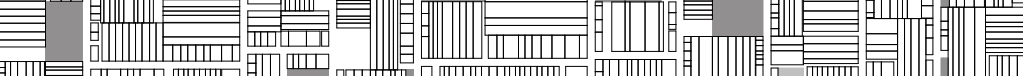

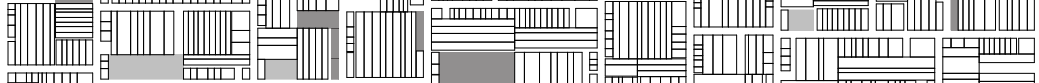

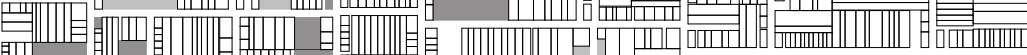
韧

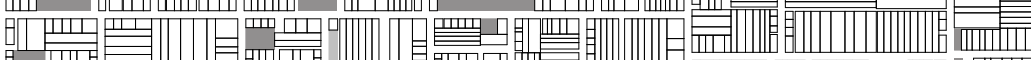
|||

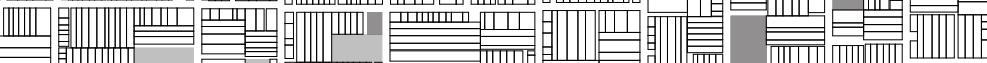

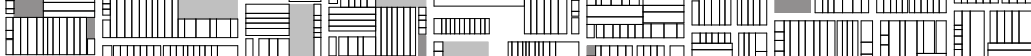
言

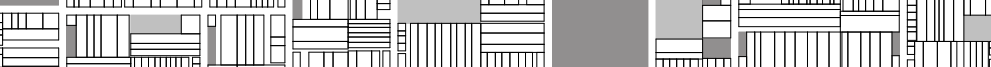
En 前员

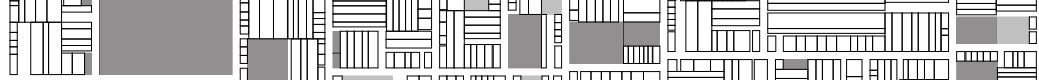

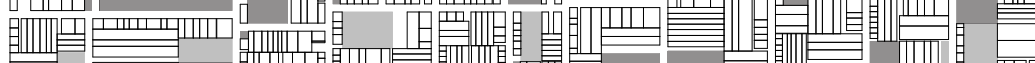

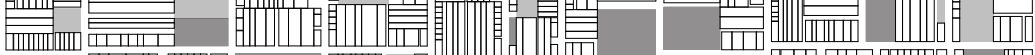

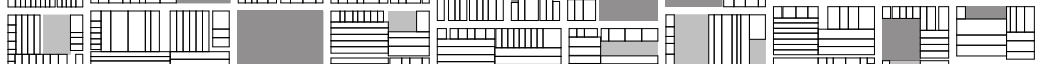

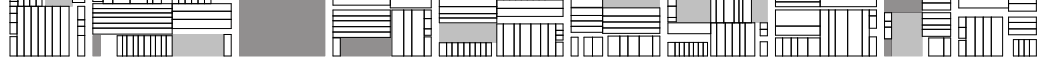
$04-$ PCGC $5 \%$

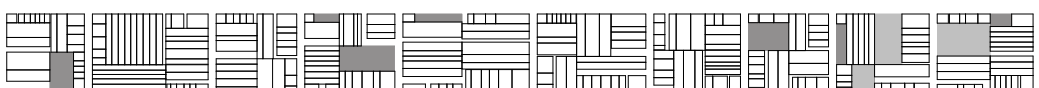

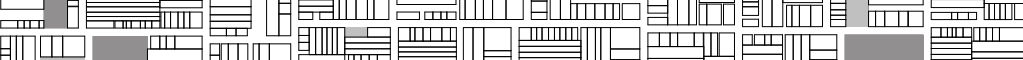

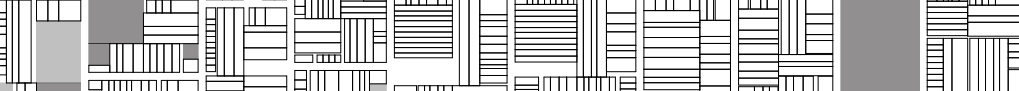
|| 目

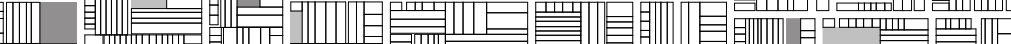

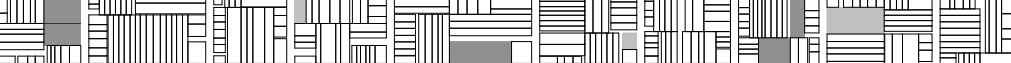

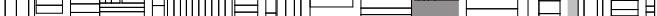

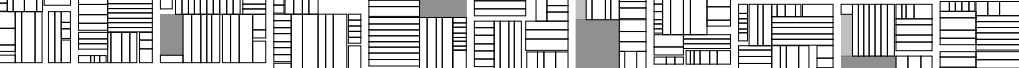
e

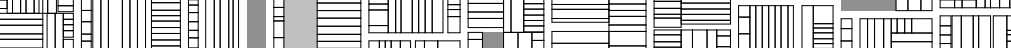

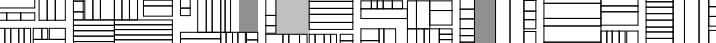

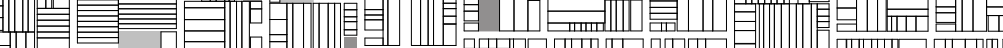

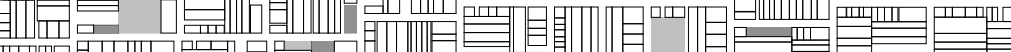

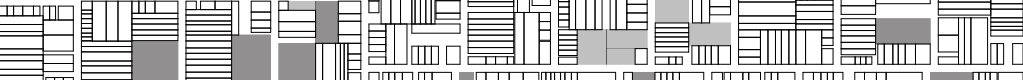

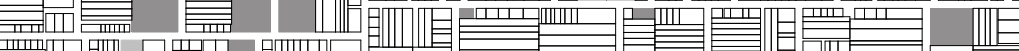

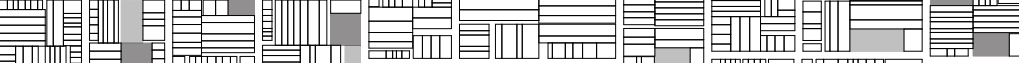

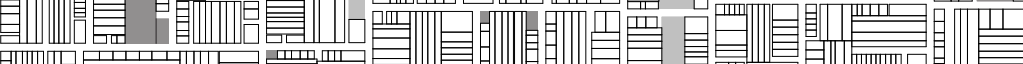
㲘睡

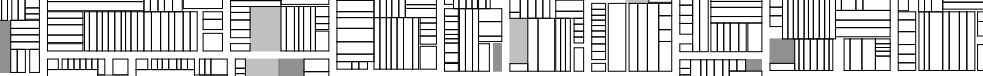
睡 $03-\operatorname{PCGC} 4 \%$

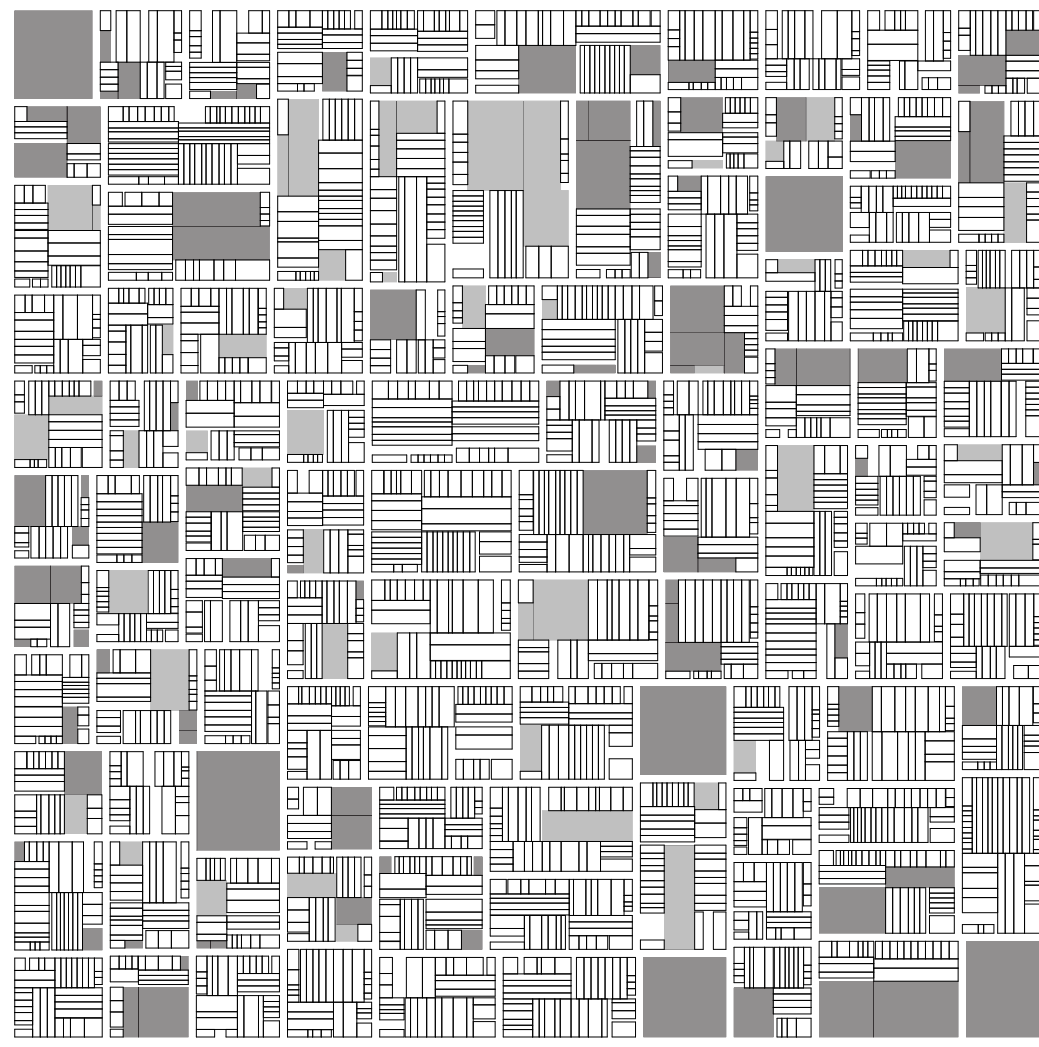

$05-\operatorname{PCGC} 6 \%$

Figure 6.04: Compilation of city generator outputs, increasing park/carpark generation 


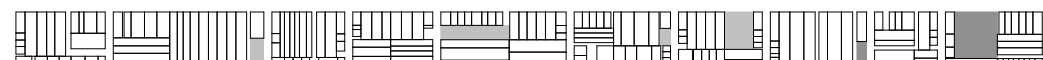
目

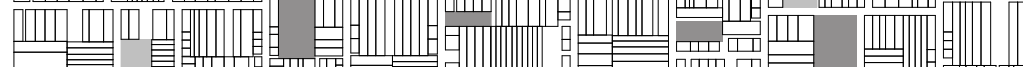

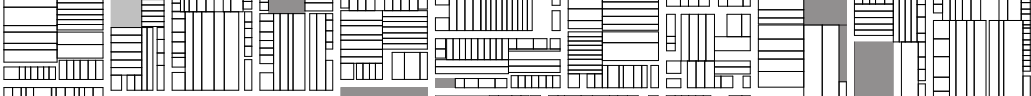

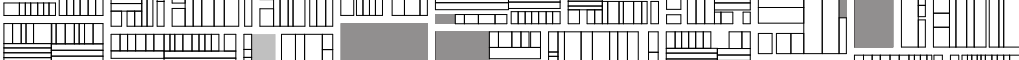

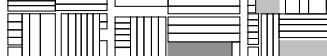

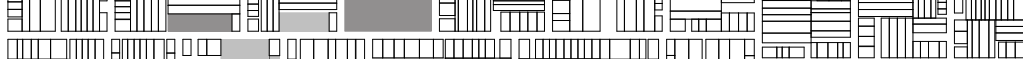

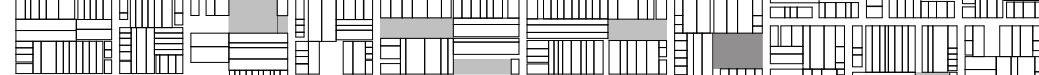

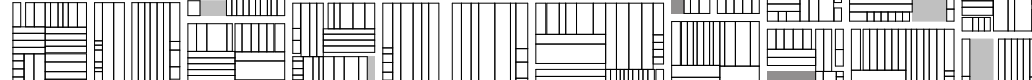

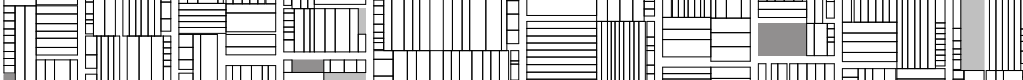

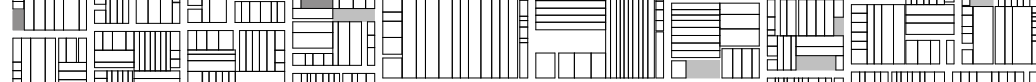
口n

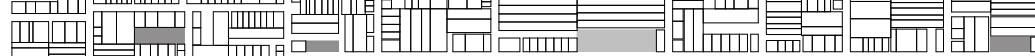

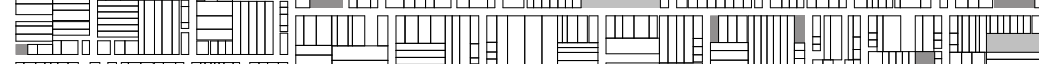
： 颗

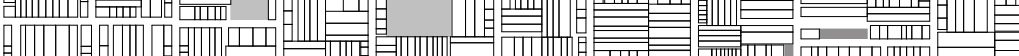

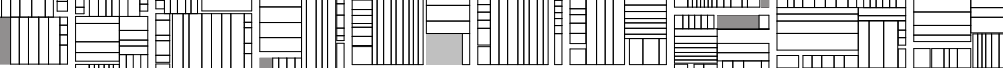

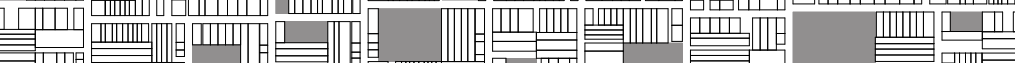

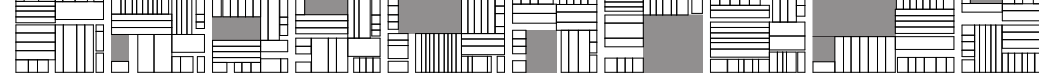
06 - Min. Range (MnR) 6 - 15, Max. Range (MXR) 10 - 40

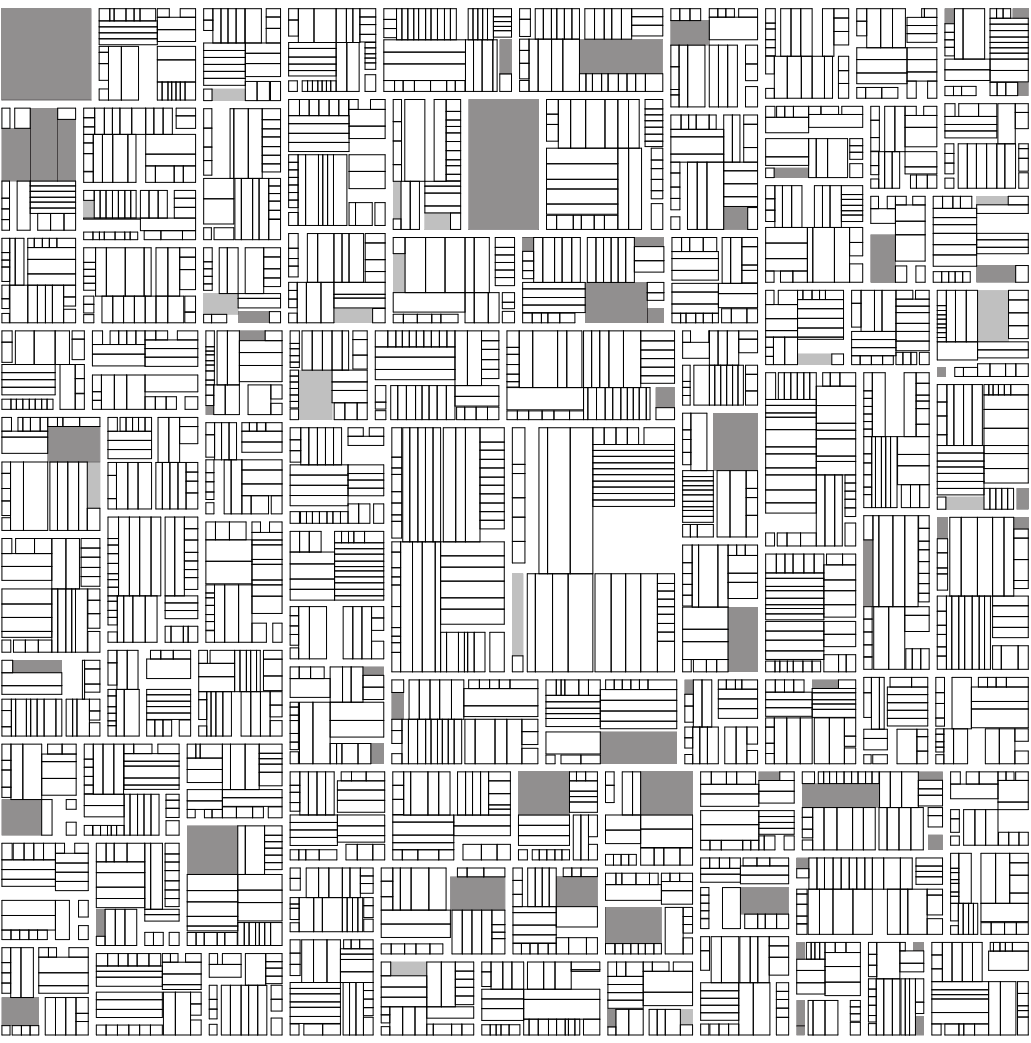
08 - MnR 6 - 21, MXR $10-50$

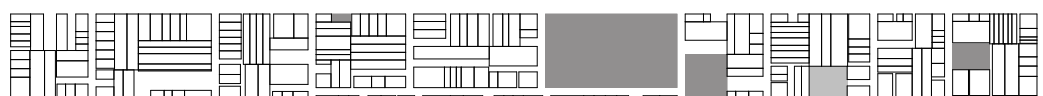

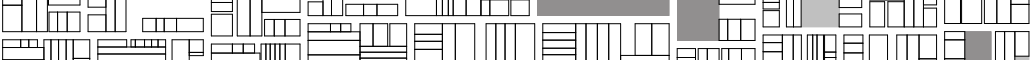

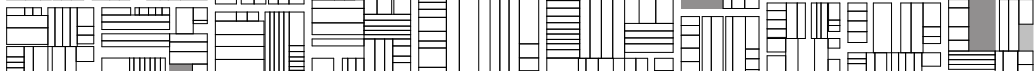
至

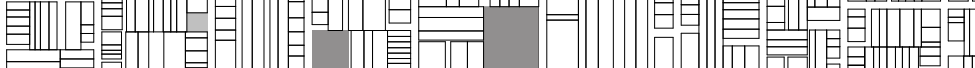

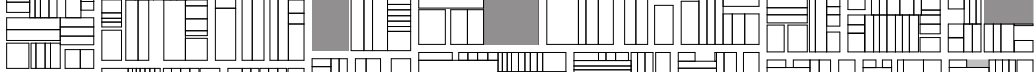

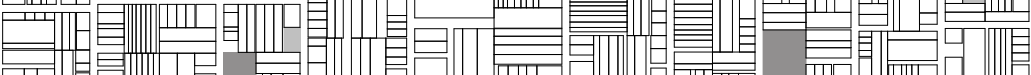

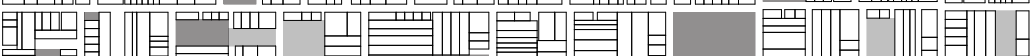

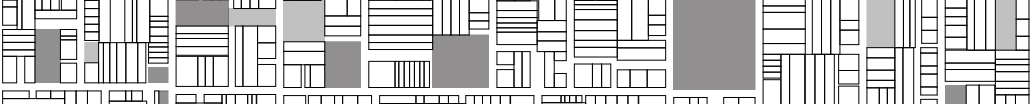

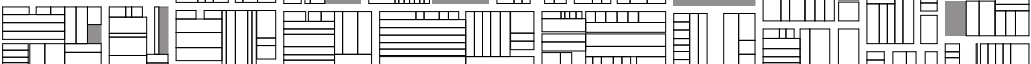

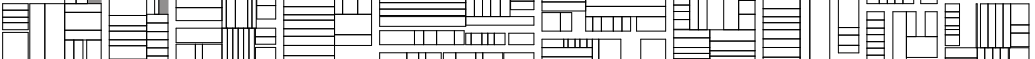

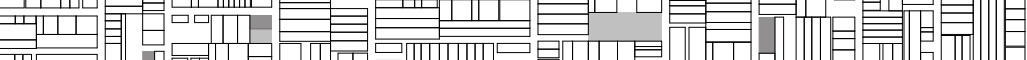
可监口

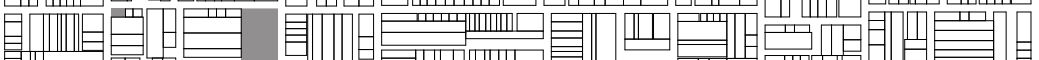

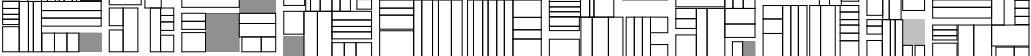

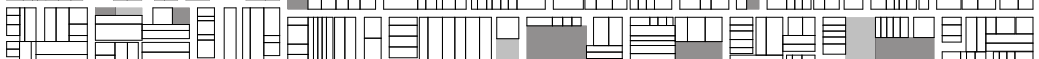

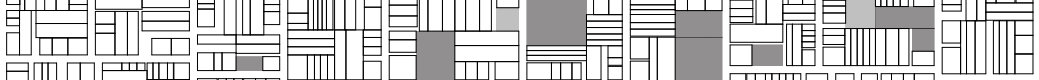

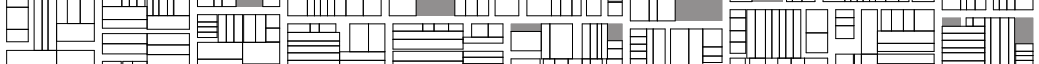

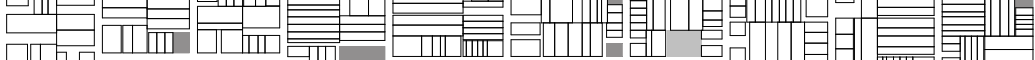

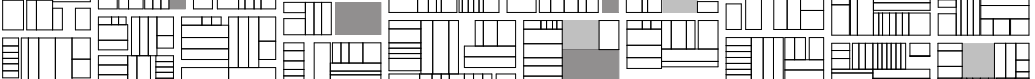

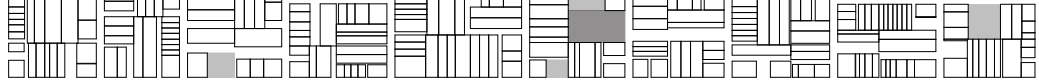
$07-$ MnR 6 - 18, MxR $10-45$

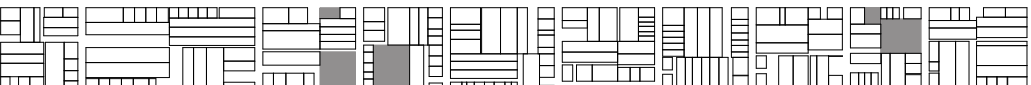
vivl

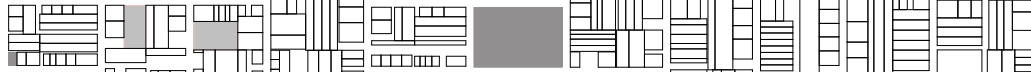
ए

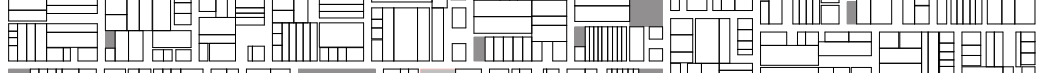
世 䀝的

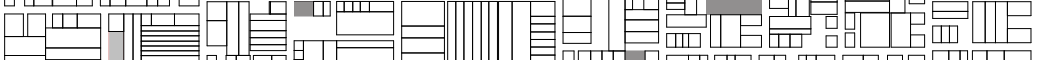

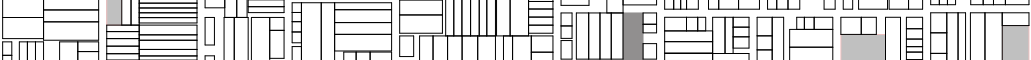
更 a

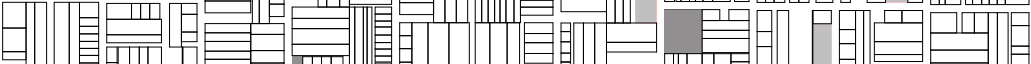

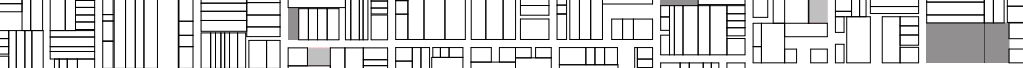

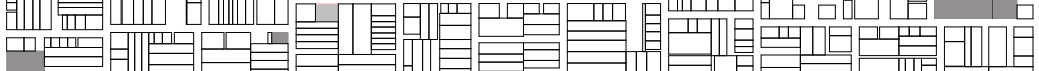

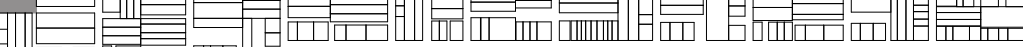

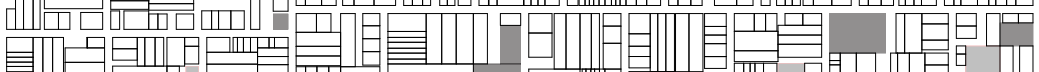
重

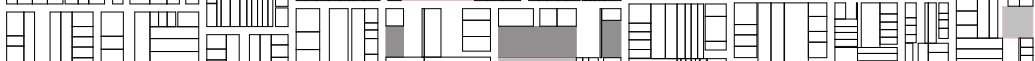

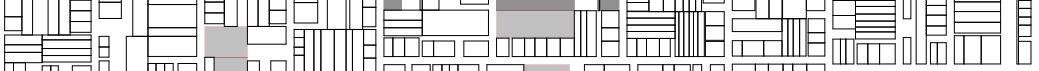

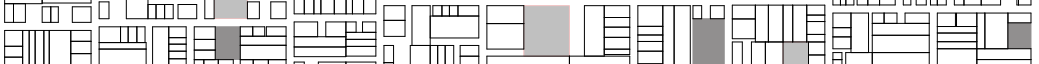

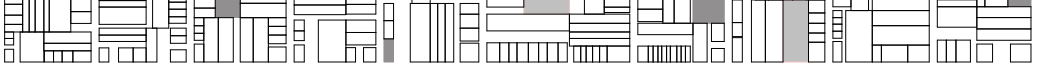
09 - MnR 6 - 24, MxR 10 - 55

Figure 6.05: Compilation of city generator outputs, lowest possible park/carpark generation, varied building width range 


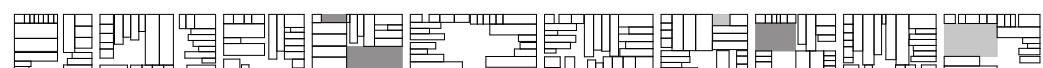

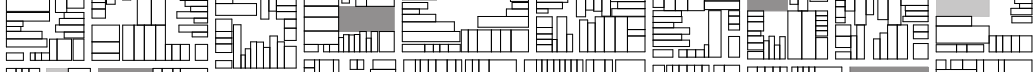

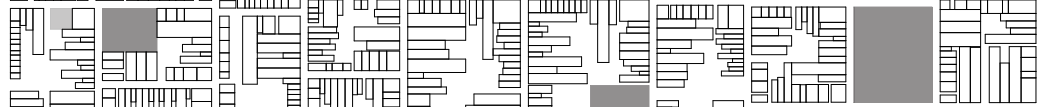

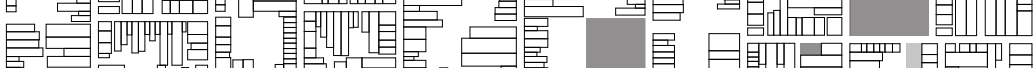

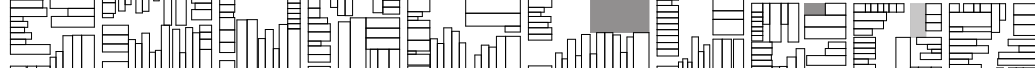
怔

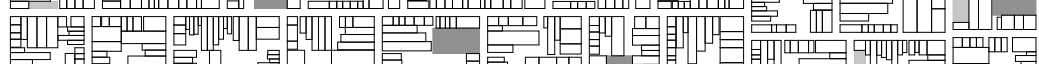

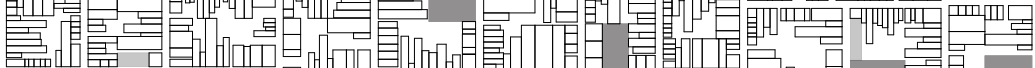

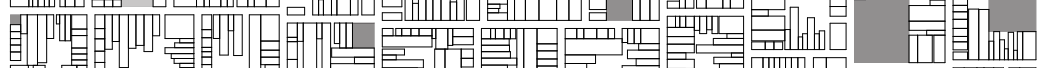

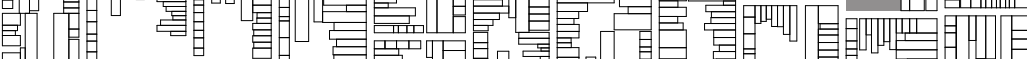

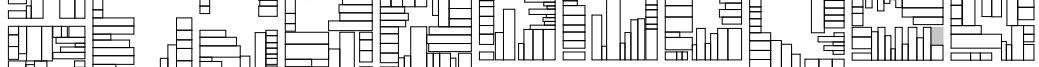

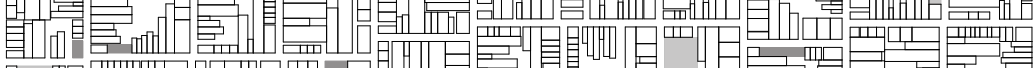

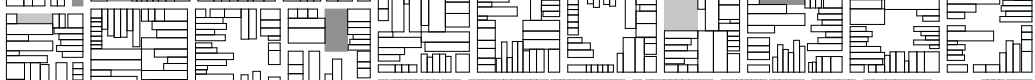

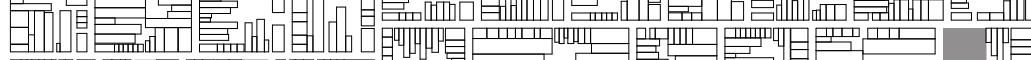

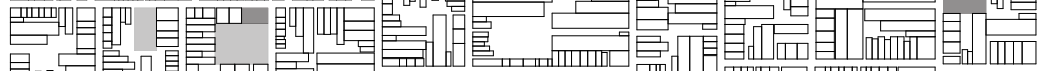

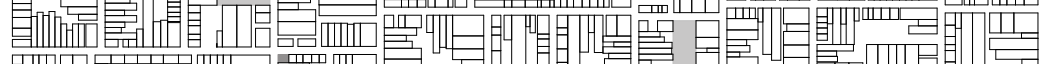

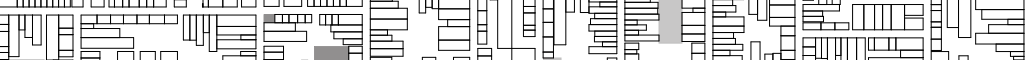

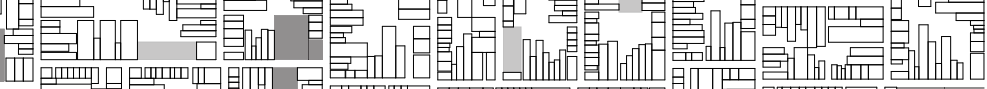
(1)

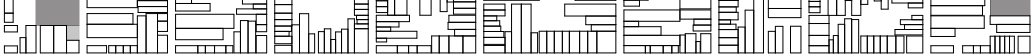
Seed 03 - Scaling Range 2.0 - 6.5

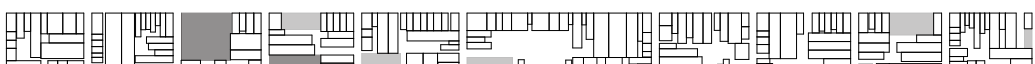

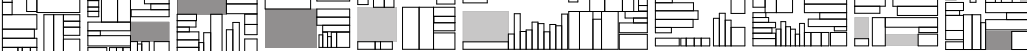

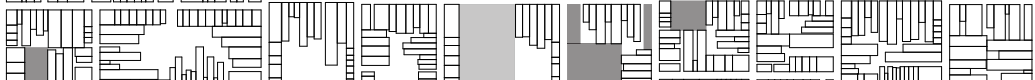

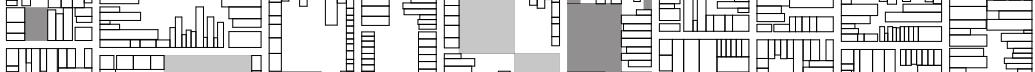

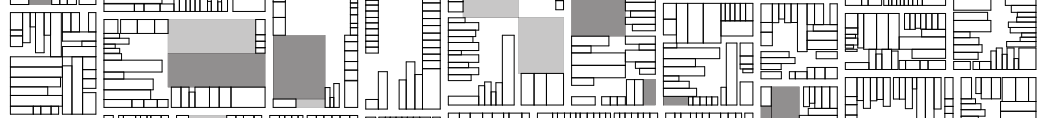

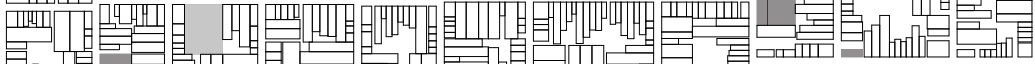

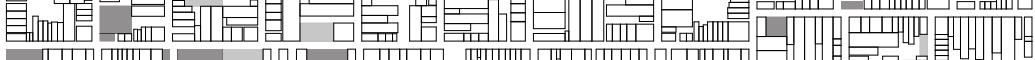

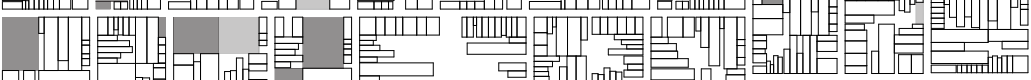

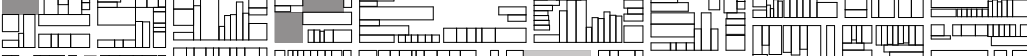

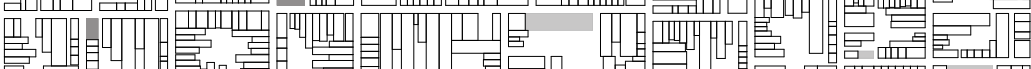

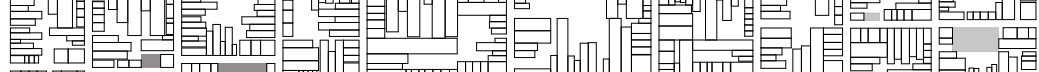

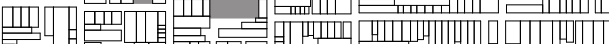

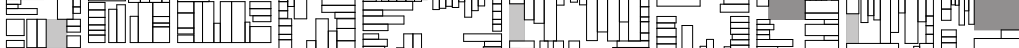

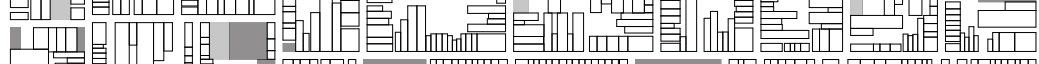

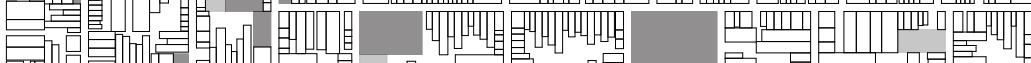

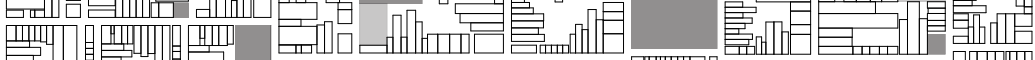
年

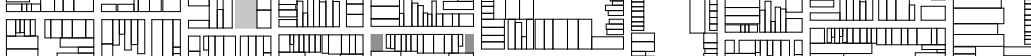
巴س 베

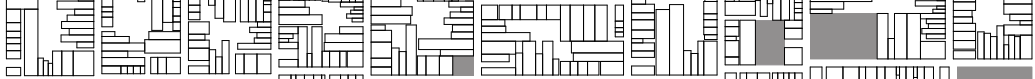

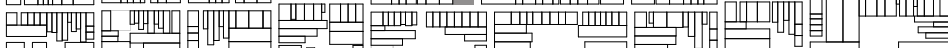

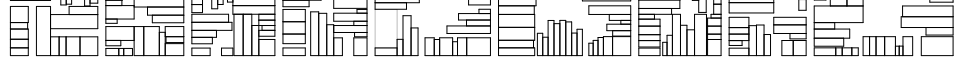

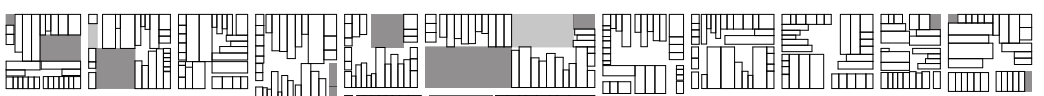

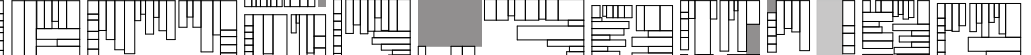

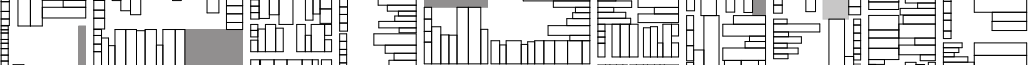

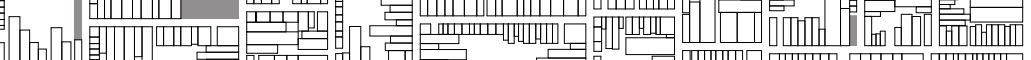

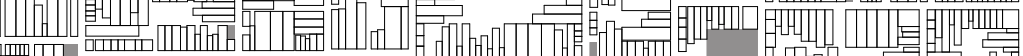

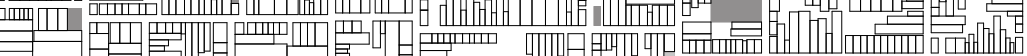

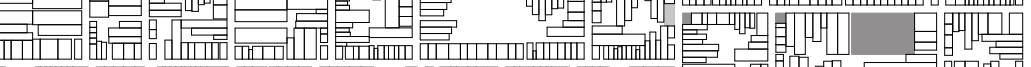

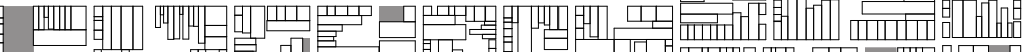

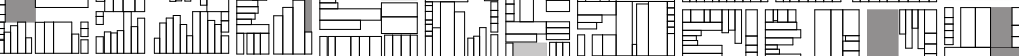

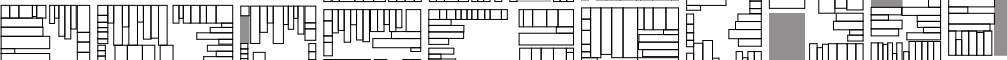

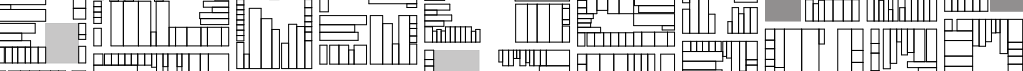

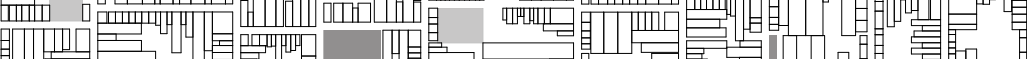
(1)

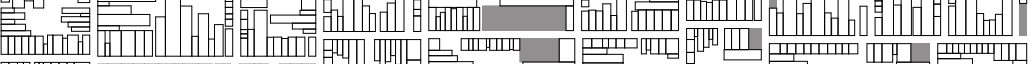
叫畞

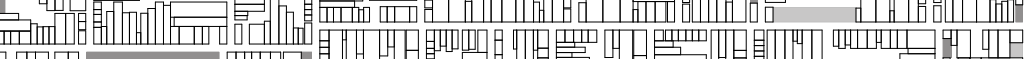
暊口

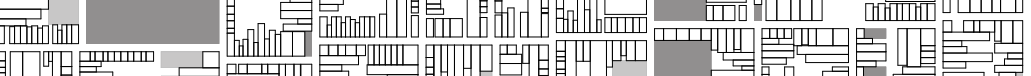

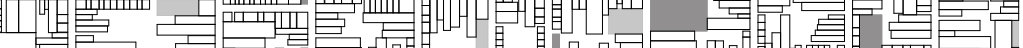

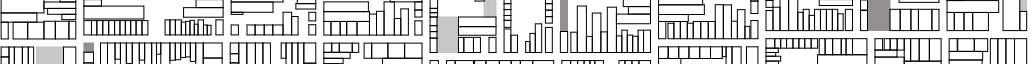

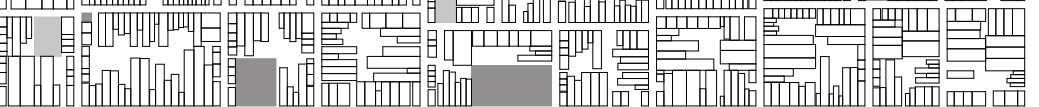
04

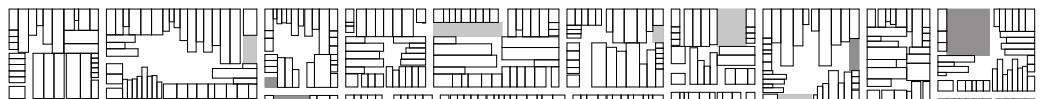

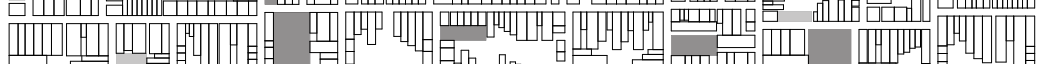

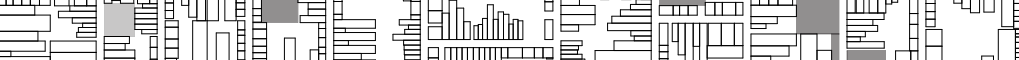

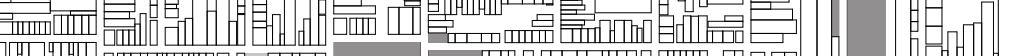

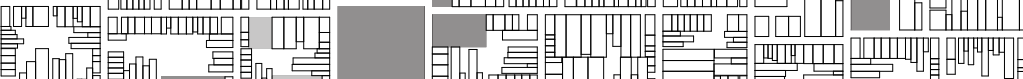

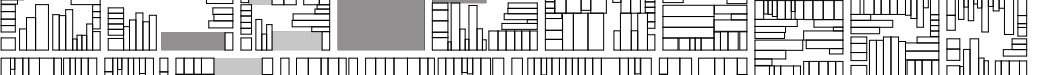

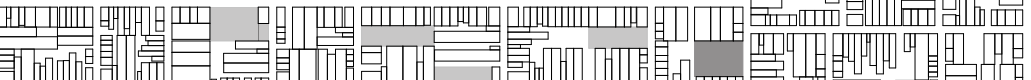

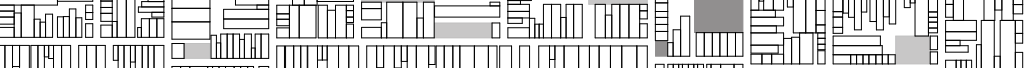

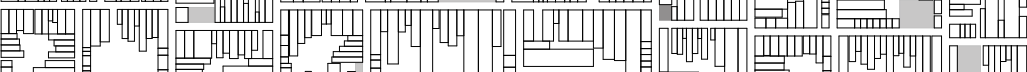

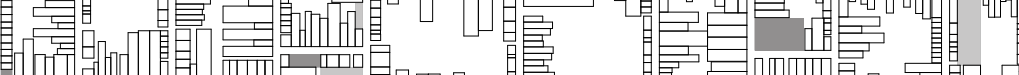

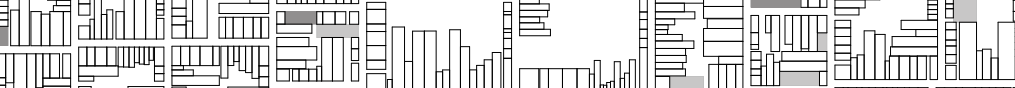

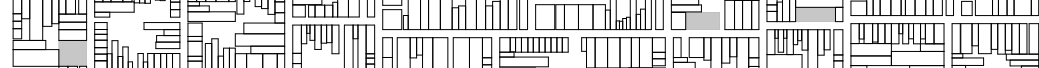

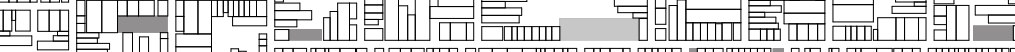
c2

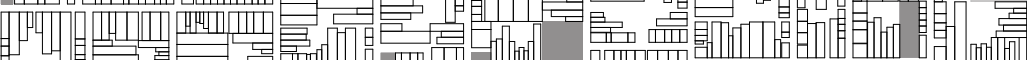

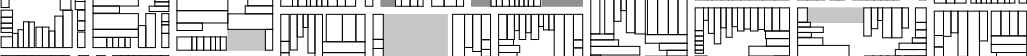

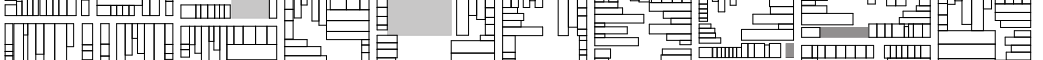

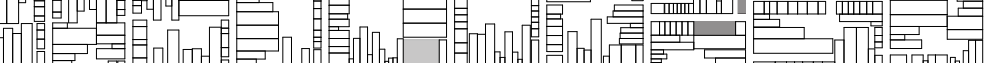

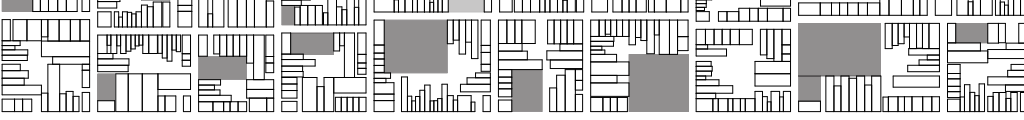
06

Figure 6.06: Compilation of city generator outputs, building scaling value added

CHAPTER SIX 


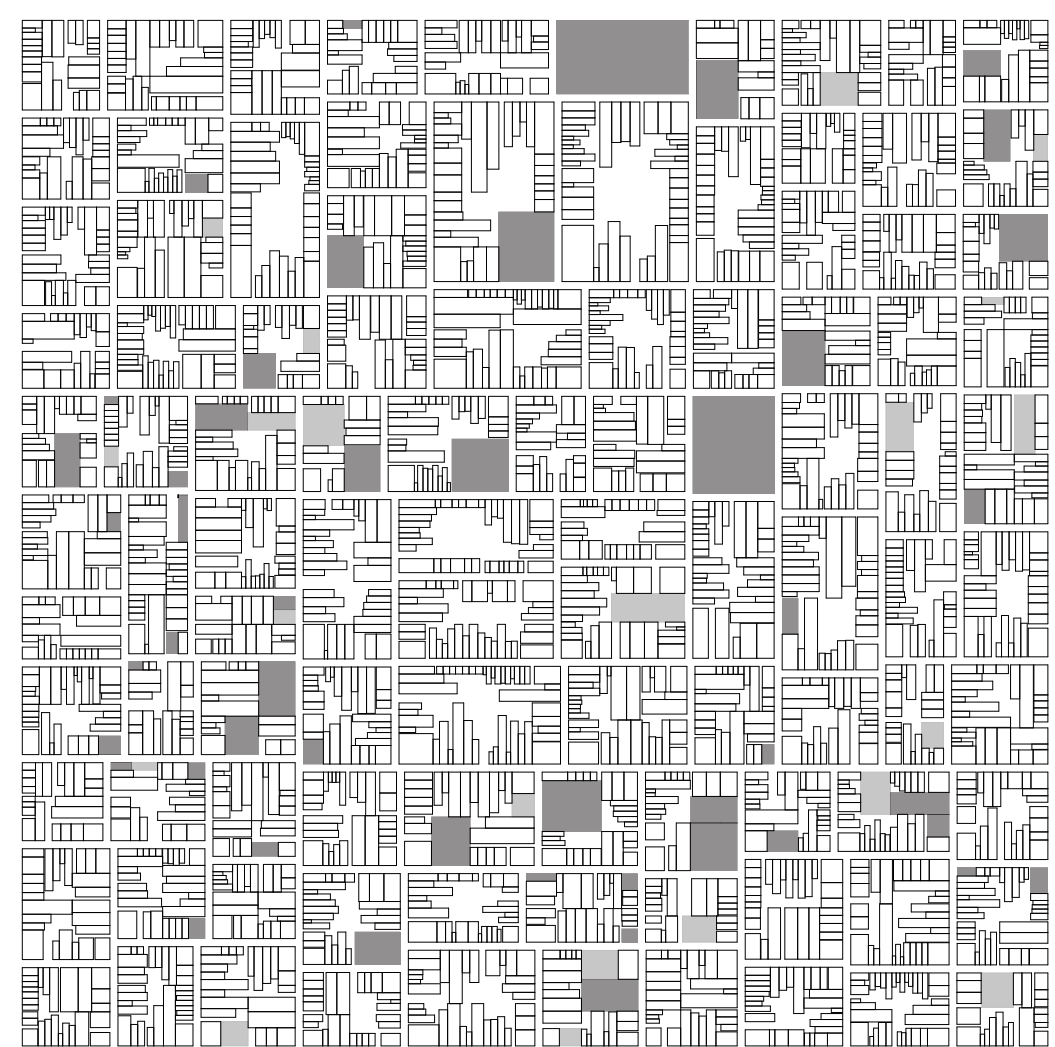
07

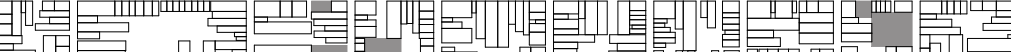

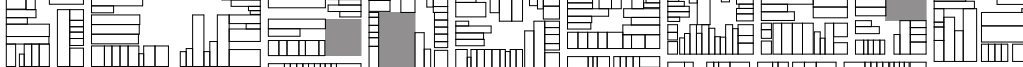

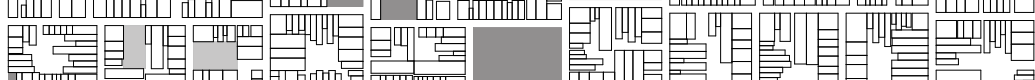

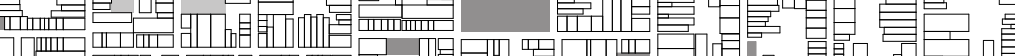

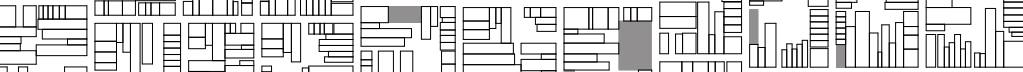
勘

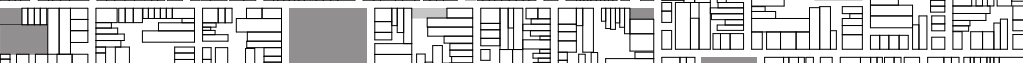

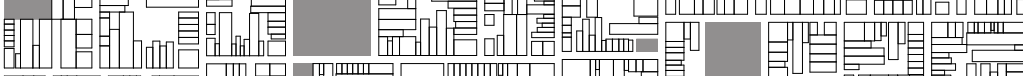

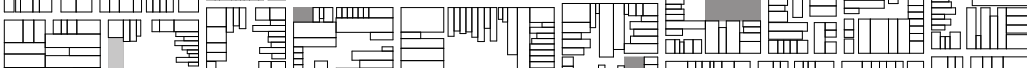

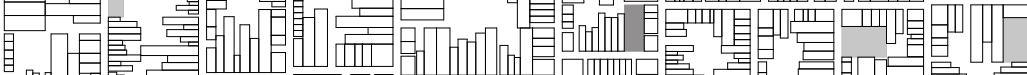

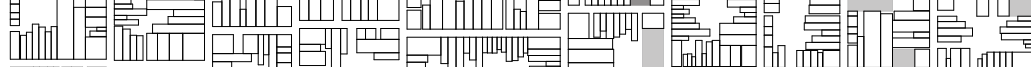

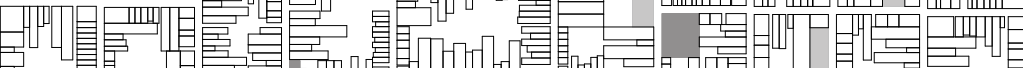

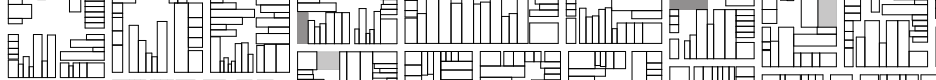

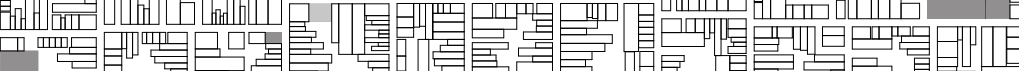

至

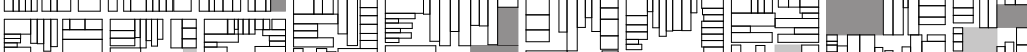

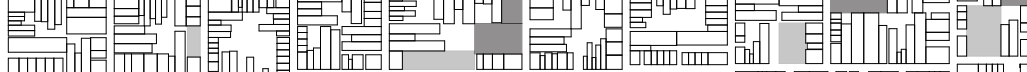

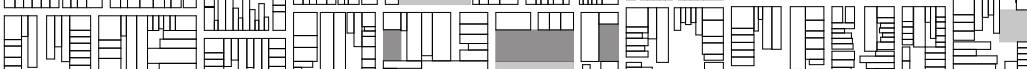

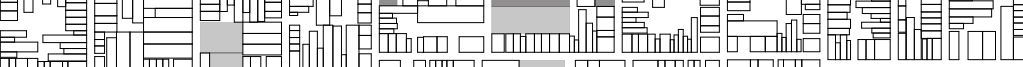

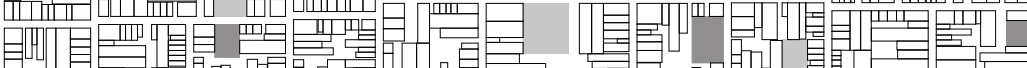

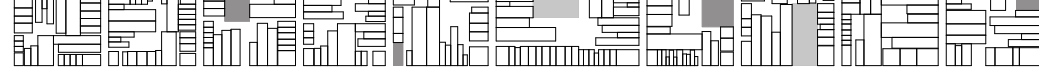
09
型|

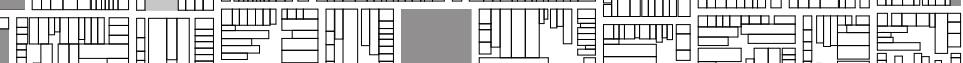

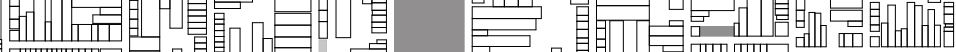
瞓鼻

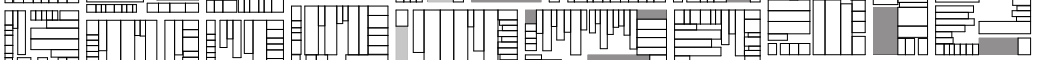

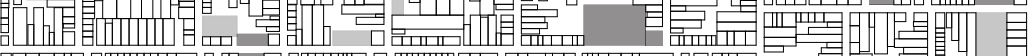

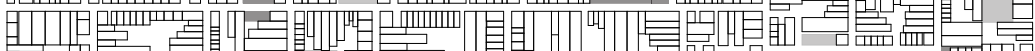

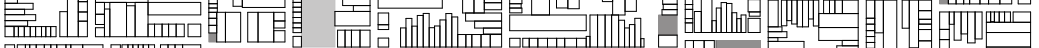

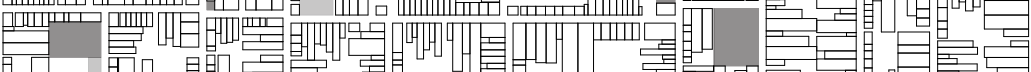
䁒 hro 目

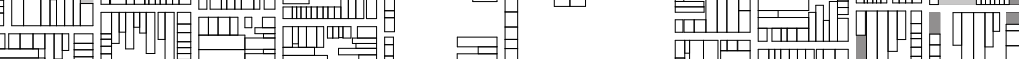

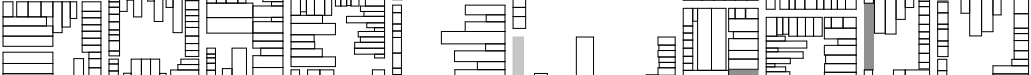

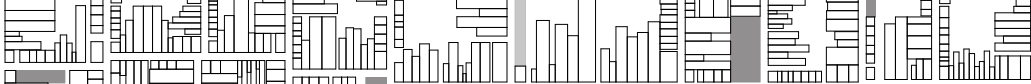
ए

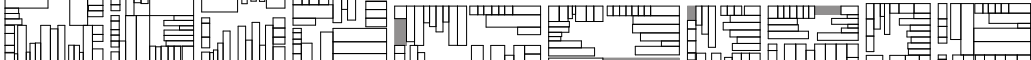

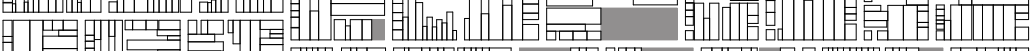

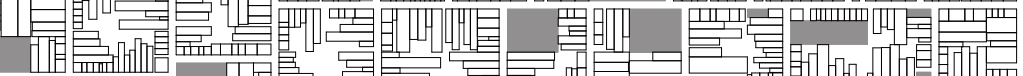

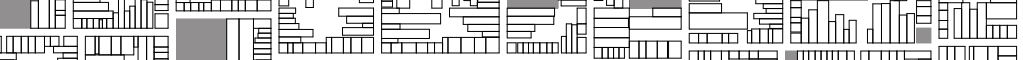

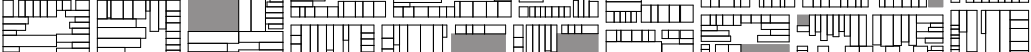

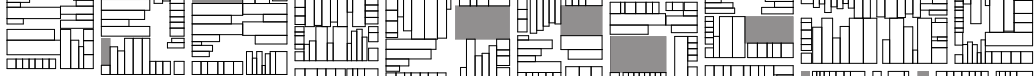

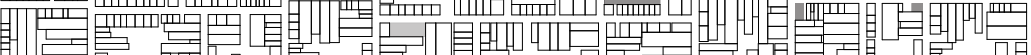

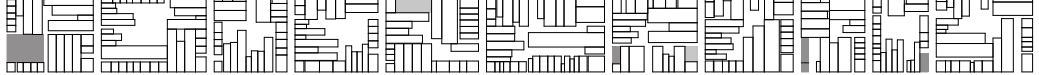
08

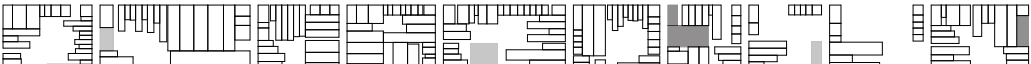

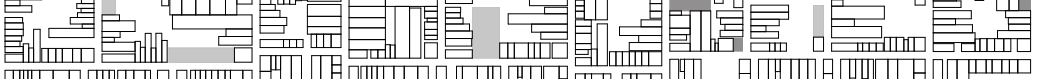

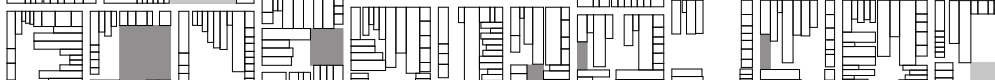

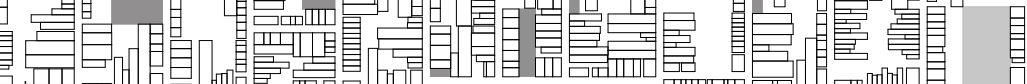

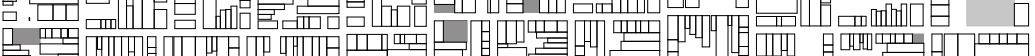

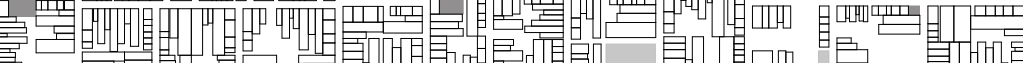

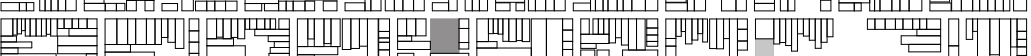

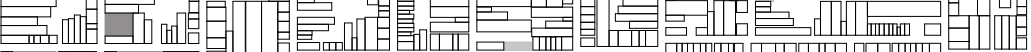

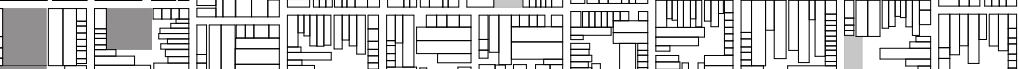

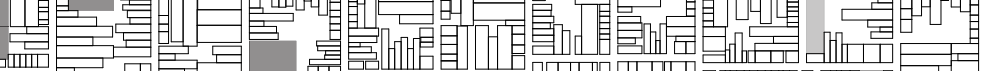

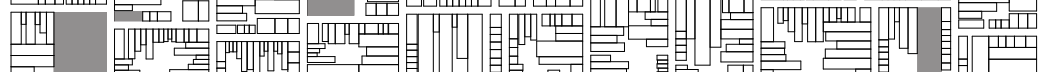

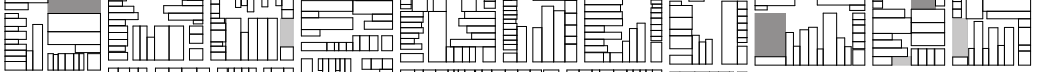

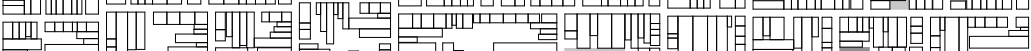

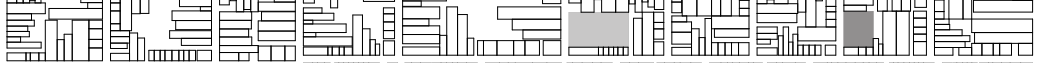

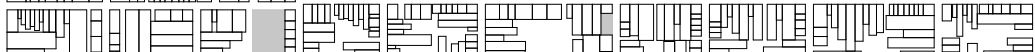

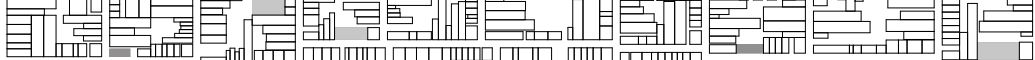

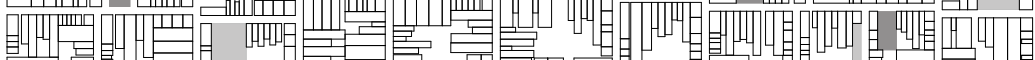

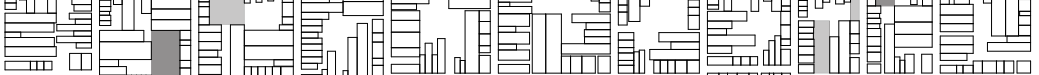

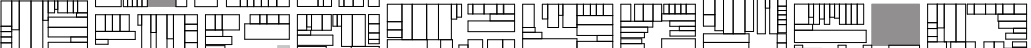

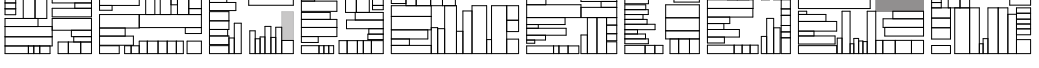

Figure 6.06: Compilation of city generator outputs, building scaling value added (cont.) 


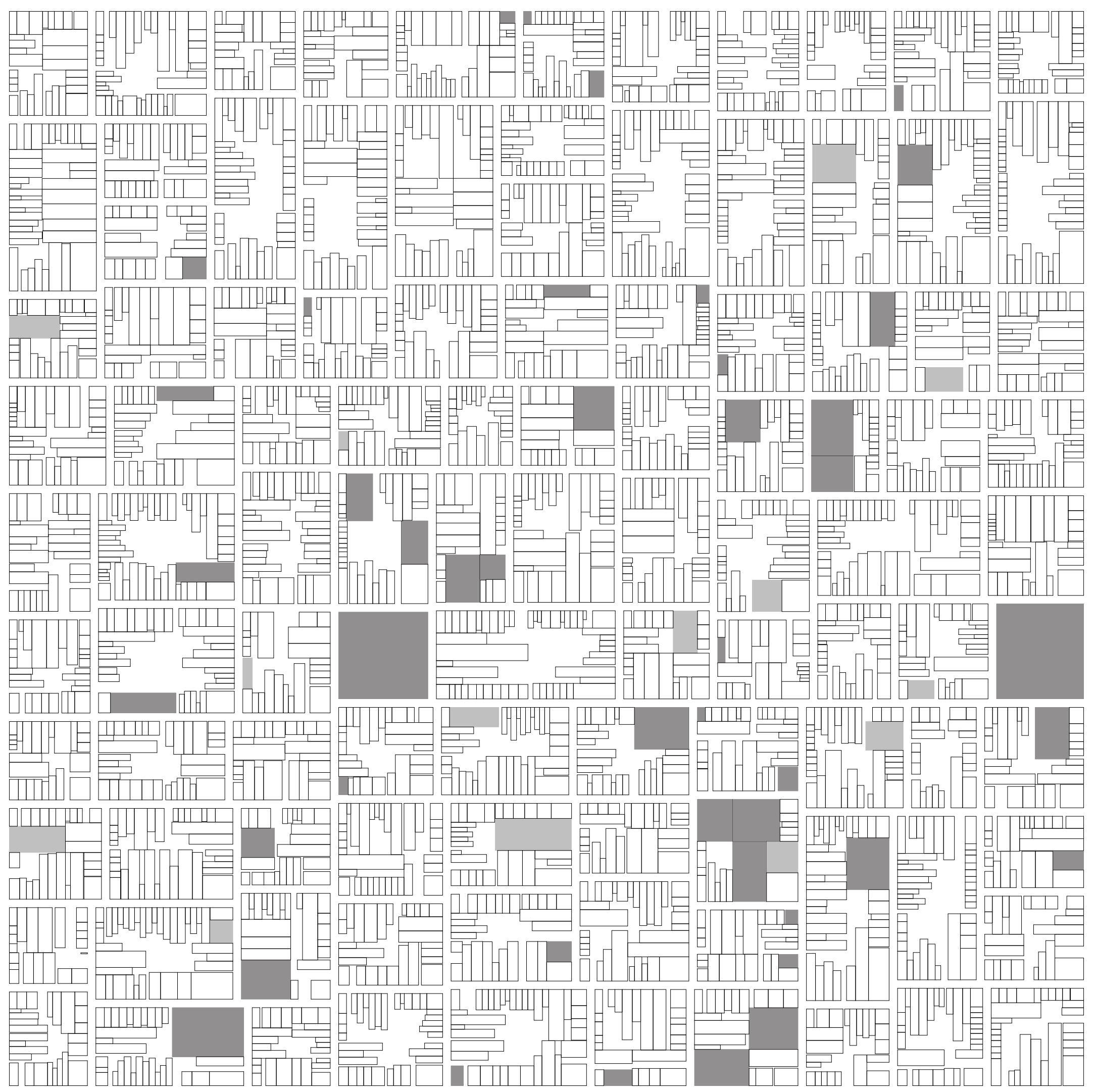

Figure 6.07: City generator output, building scaling range 2.0 - 6.5 added, Seed 02

CHAPTER SIX 


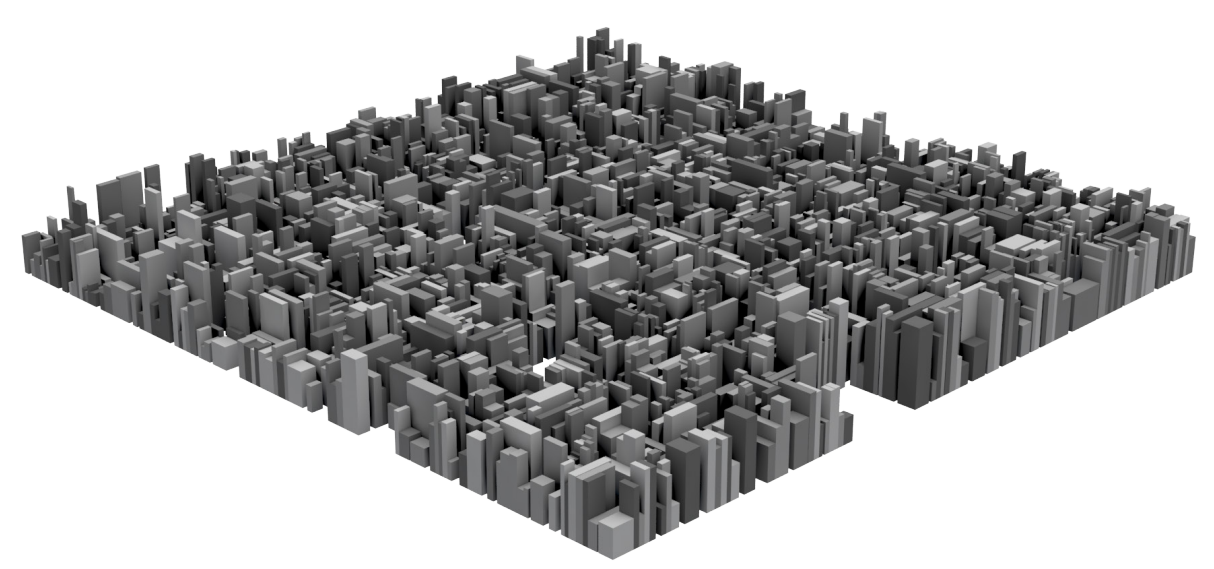

Seed 02: Height 15 - 200, Value Shuffle (Jitter) 0.5\%

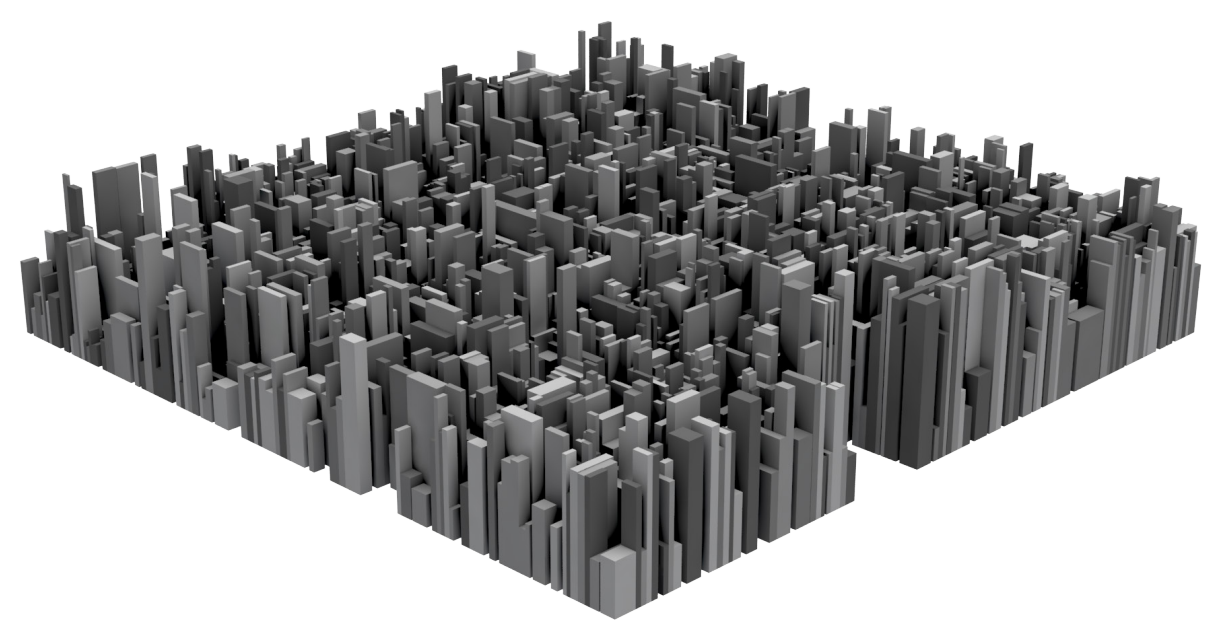

Height $15-400$

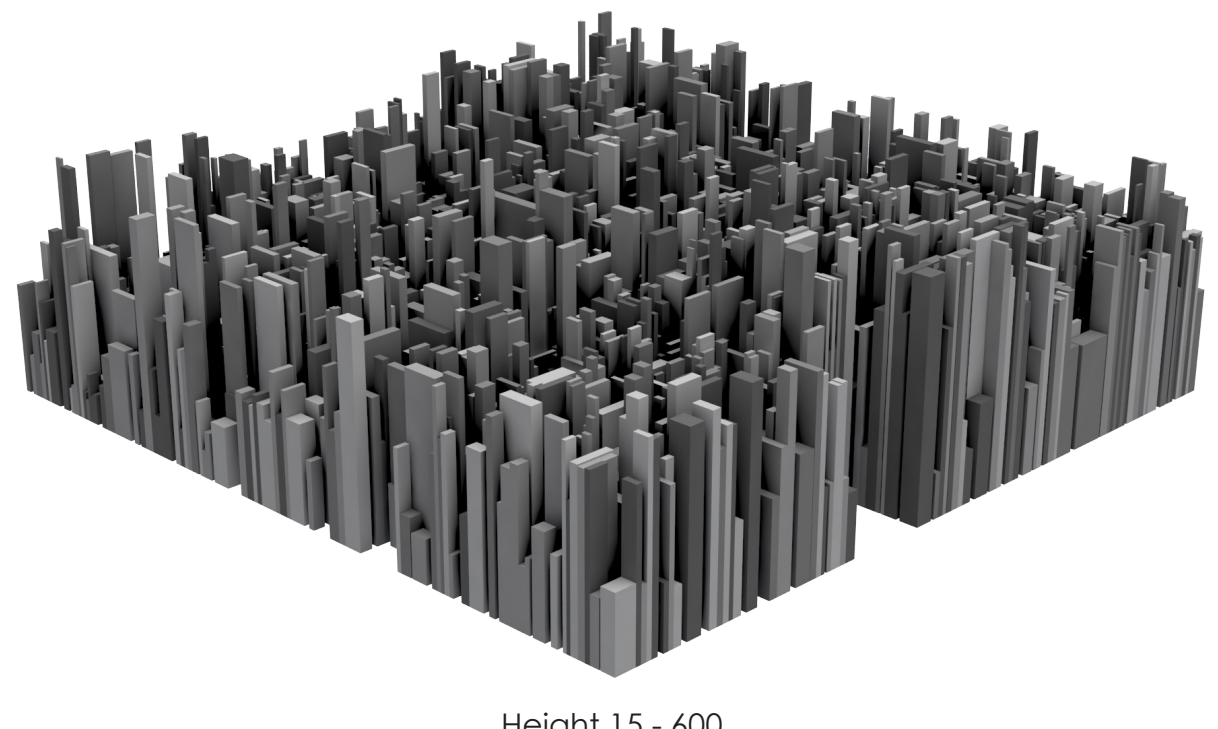

Height $15-600$

Figure 6.08: Compilation of city generator outputs, including jittered values and varied extrusion ranges, Seed 02 


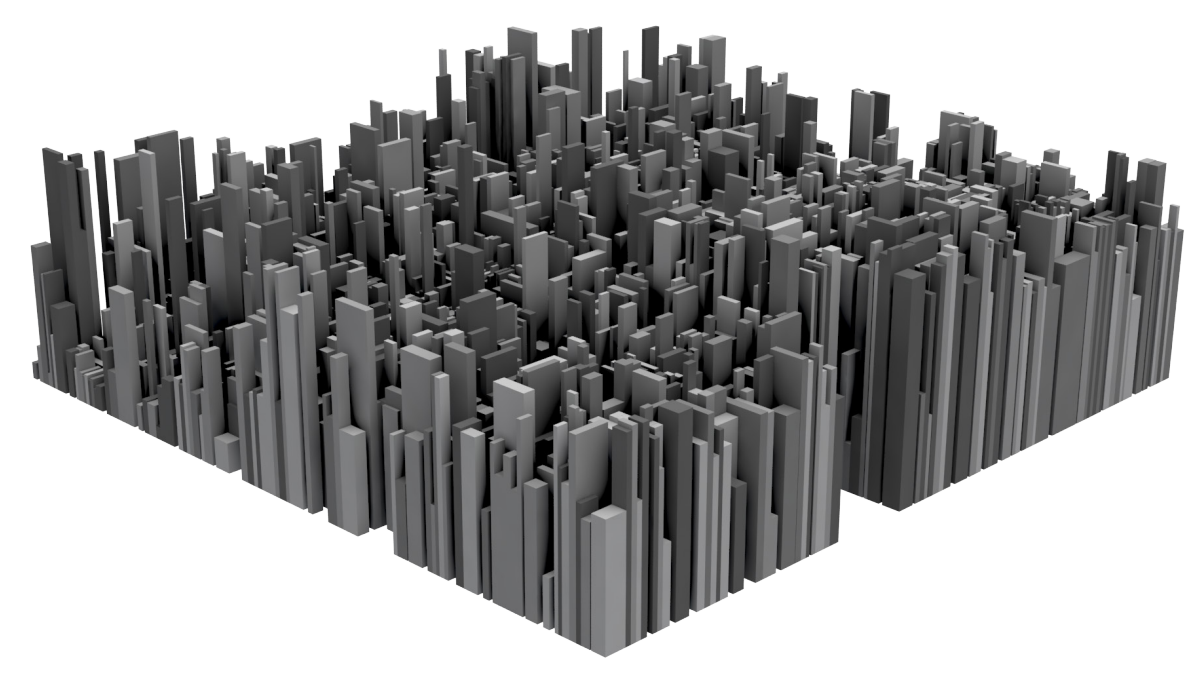

Height 15 - 600, Jitter $0.25 \%$

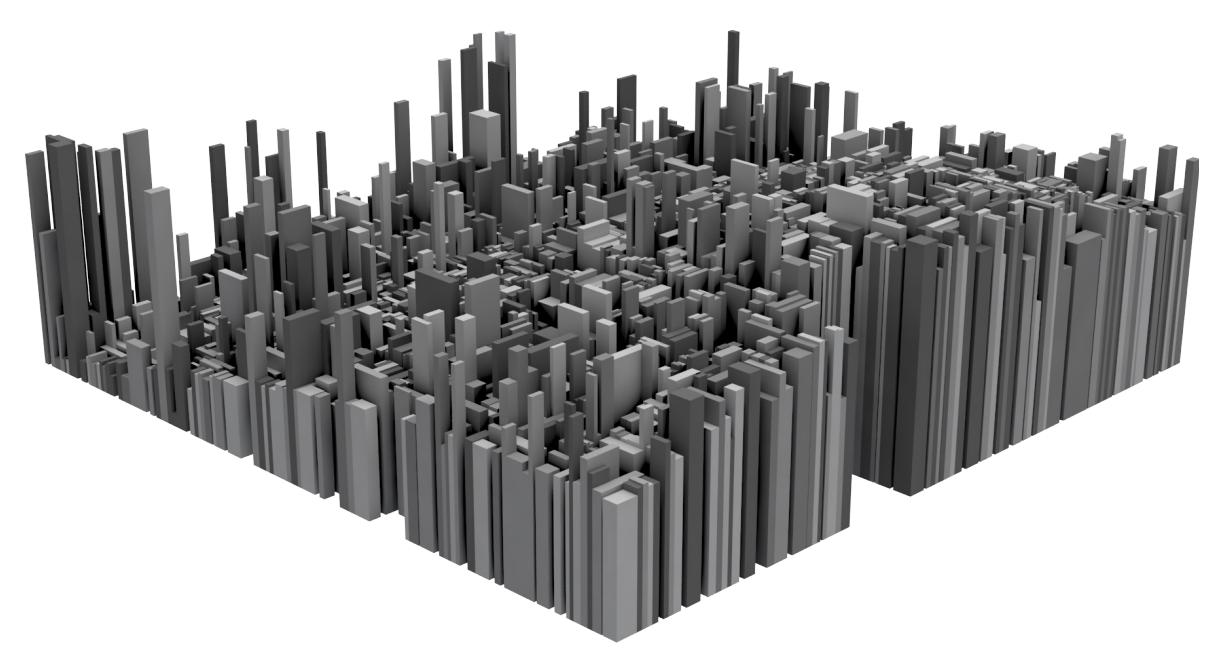

Jitter $0.0625 \%$

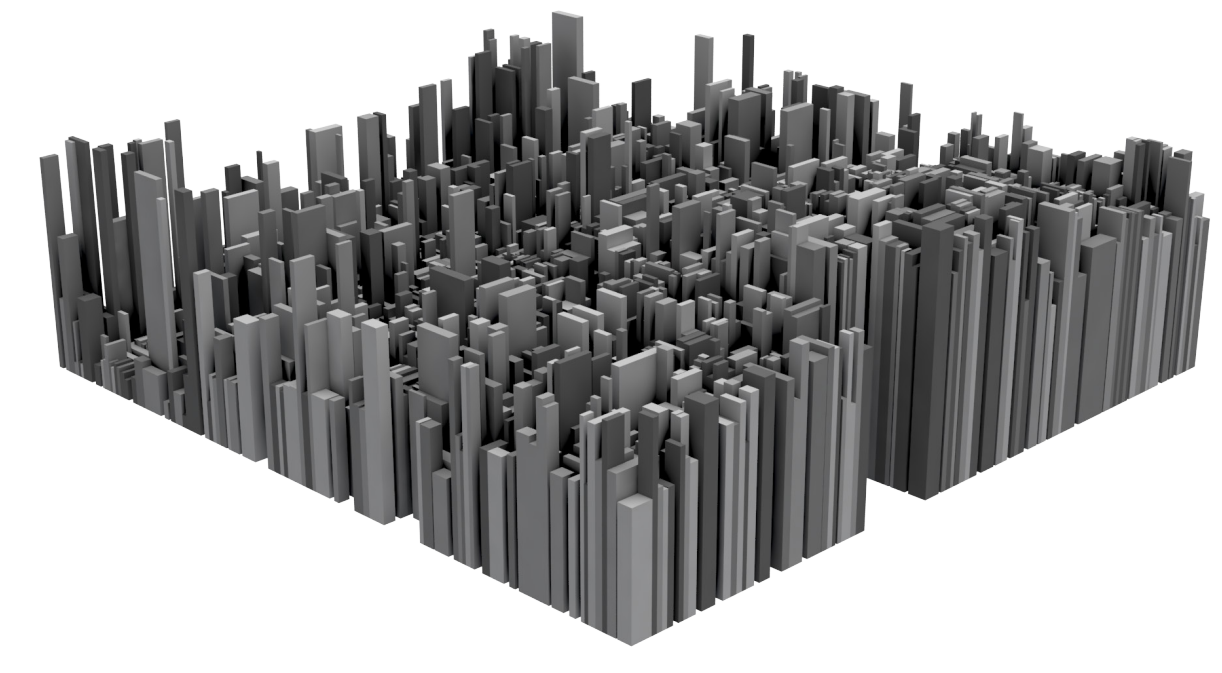

Jitter $0.125 \%$

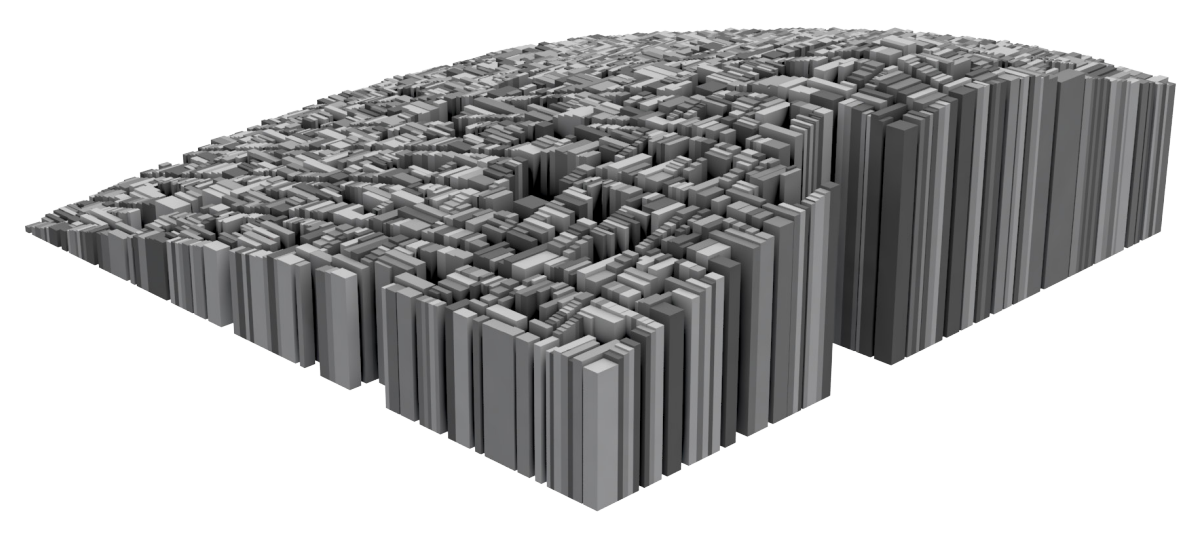

Jitter $0 \%$ 

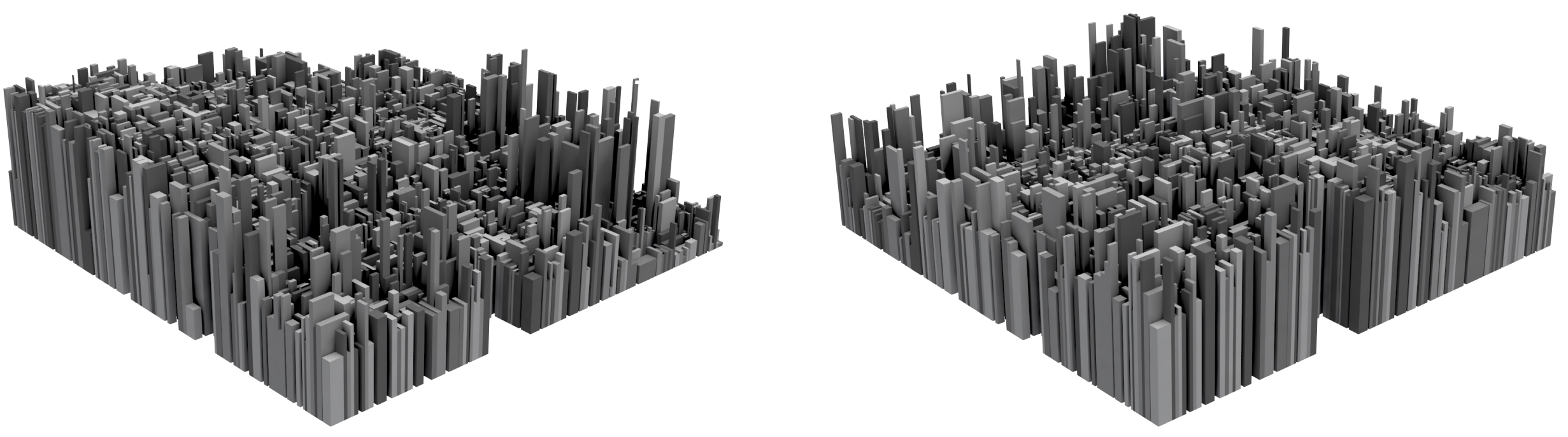

Height 15 - 600, Jitter 0.0125\%, Attractor Seed (AS) 01

AS 02
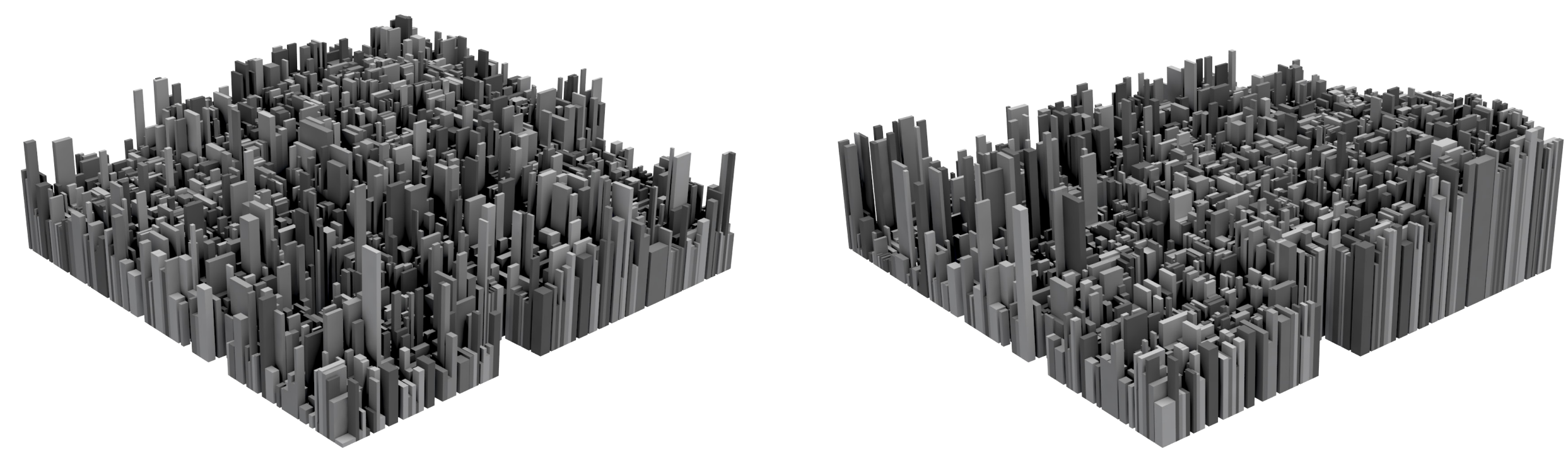

AS 03

AS 04 


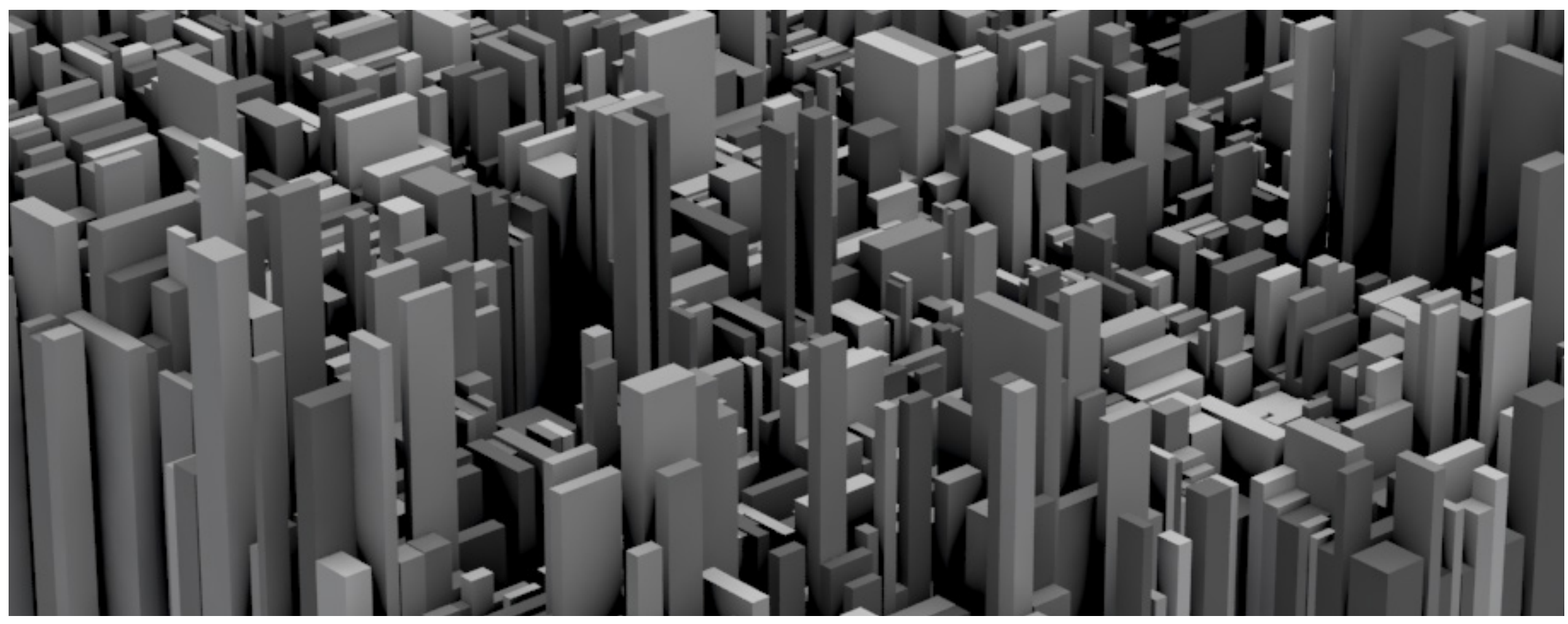

Height 15 - 600, Jitter 0.0125\%, Attractor Seed (AS) 01

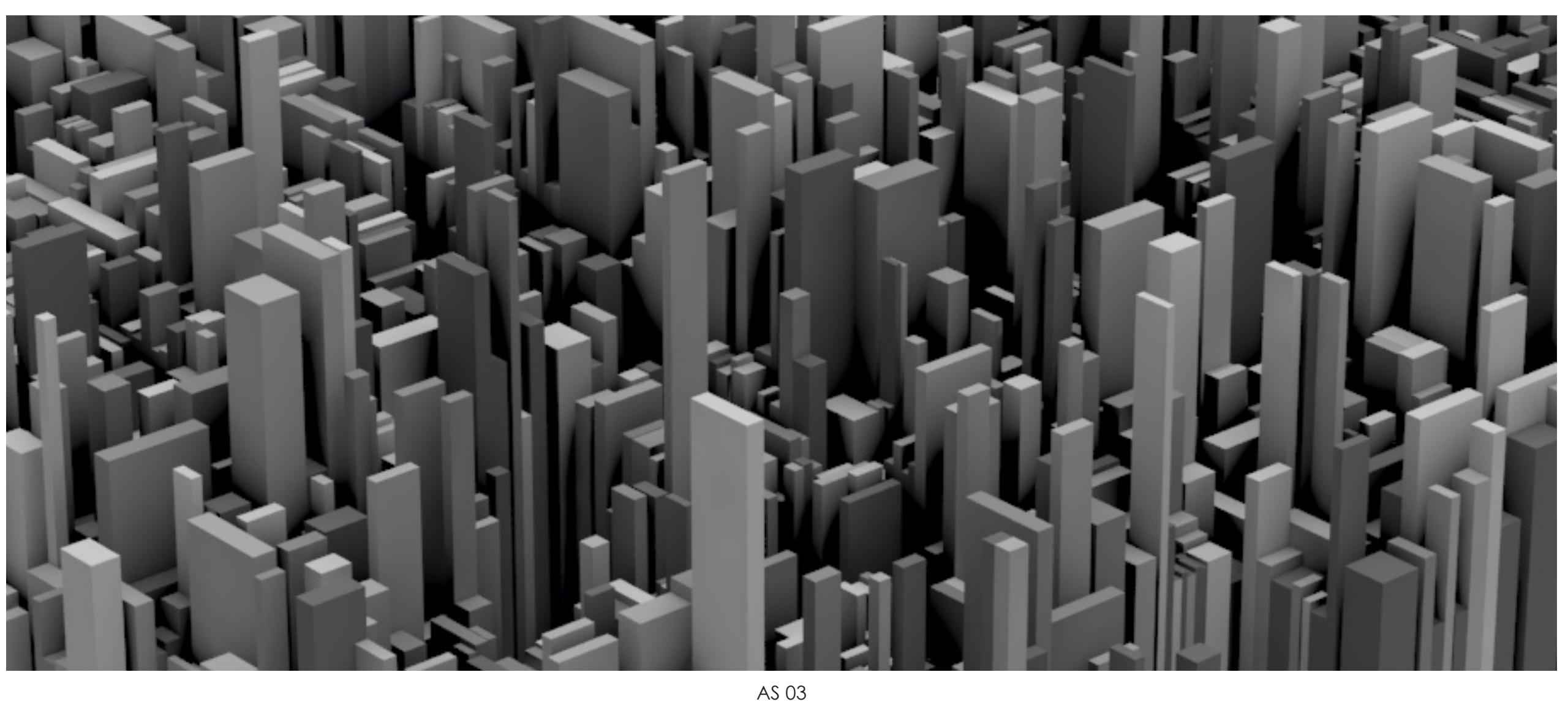

Figure 6.11: Compilation of city generator outputs, including fixed jittering values, fixed extrusion range, and varied attractor point placement, 200\% zoom, Seed 02

CHAPTER SIX 

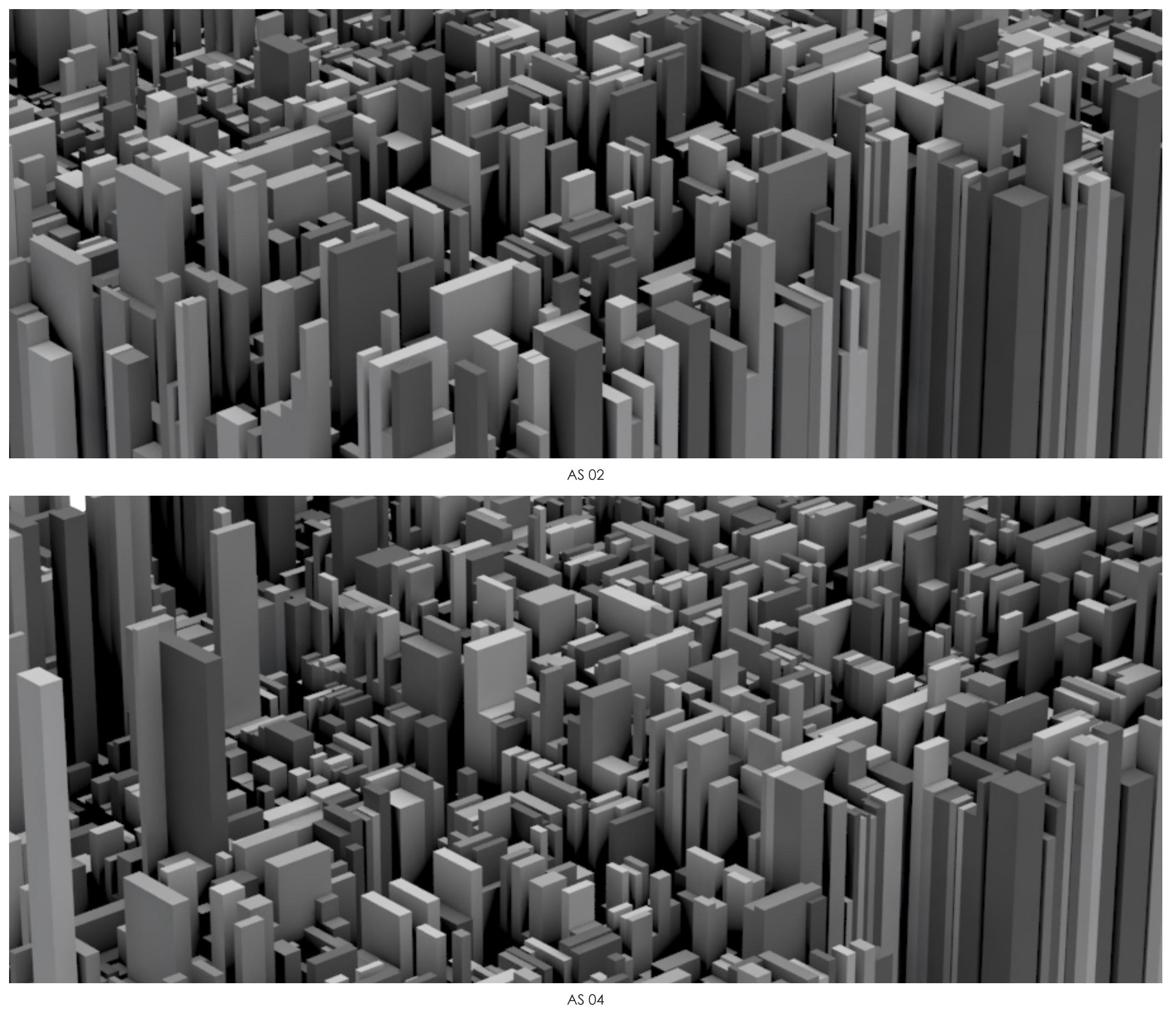

Figure 6.11: Compilation of city generator outputs, including fixed jittering values, fixed extrusion range, and varied attractor point placement, $200 \%$ zoom, Seed 02 (cont.) 


\section{CONCLUSION}

This design led research focused on the practical application of visualprogramming software as a means of generating architectural geometries. Primarily using Grasshopper, this research demonstrates that procedural generation techniques and ideas can be accessible and practical to relevant industries. Through this process the research has also established that the software has a distinct range of limitations. Whilst all the research goals have been met, this thesis raises doubts over whether Grasshopper is the best platform for dealing with them.

Mastery of visual programming was a major driving force of this research, and a decision was made to work solely within Grasshopper. As a tool, Grasshopper is fundamentally important within the architectural industry. Furthermore, working with a visual programming interface allows this research to facilitate architectural discussion within the fields of film and interactive media. The application of coding or more powerful parametric tools, such as Houdini, may have helped to further this research, but the barrier to entry with either of these approaches would have made it unreasonable.

One lesson from this research is that every system can be improved, or approached in a different way. This can be the result of familiarity, exposure to new tools/ideas, or simply the realisation that there is a more efficient way of producing the desired outcomes. The process of rebuilding a system should be considered a requirement of its construction. Inevitably, the first iteration of any system will be haphazard as a variety of problems are both identified and solved. Any further iterations act to resolve these issues, provide optimisations, and improve the understandability and reusability of the Grasshopper canvas. It would be faster to create a system that works the first time around, but this requires a considerable degree of foresight. This became apparent at many stages of this research, where systems had not been built in a way that allowed for the simple introduction of further parameters. Retroactively introducing additional parameters then became a daunting task, involving modifying large swathes of the existing canvas. Generally speaking, the fastest option is to completely rebuild the system with a greater number of considerations in mind.

A feature not attempted in this system is the ability to single out entire blocks, or footprints. The purpose of this functionality would be to allow for the placement of highly detailed "hero" blocks, buildings, and so forth. While this has not been specifically addressed in the work, it is entirely possible to implement through list manipulation. More specifically, the addition of hero buildings falls outside the scope of this research.

Varying degrees of complexity have been included at multiple stages throughout the research including the columns, and roof forms in the extruded city generators. Both of these situations demonstrate the idea that if detail can be generated procedurally for one element, then it can be for any other. If a system is capable of generating a column, then another system can generate a door, windows, balustrades, or any other architectural geometry. However, displaying each of the possible geometric outcomes would add little to the research. In this context, such a level of detail is both unnecessary and impractical. This is particularly the case when working at the scale demonstrated in the final city generator. Instead of displaying a higher degree of mastery within the software, the inclusion of higher visual detailing would simply highlight that a greater amount of time had been spent constructing the system. To properly include more visually complex buildings in a meaningful way would require more processing power and a true procedural system. However, this would also raise questions regarding appropriate levels of detail and representation versus visualisation.

Each system is capable of generating hundreds, if not thousands, of unique outputs, depending on how the system is structured. In each instance, this research has only been able to document a limited number of outputs. The sheer number of outputs means that a large number of outputs can are generated, and then only the best outputs displayed. However, this also makes optimisation and parameter selection difficult. The number of possible outputs makes it difficult to establish combinations of parameters produce the most interesting, aesthetically pleasing, or valuable outcomes. One way to figure this out in earnest would be through data collection, measured against a specific set of variables. Recording this data would allow for the more successful outcomes to be produced, within a set framework. Within this investigation, values have been selected for arbitrary reasons, based on previous work, or because of a visual similarity to real world examples. Optimising these parameters to respond to environmental conditions, surroundings, building age, and so forth would present an exciting path for further investigation.

Overall, this work establishes a foundation for other researchers to build upon. Grasshopper is capable of dealing with immense complexity, but it is not the most appropriate way of doing so. Subsequently, this work provides an accessible logic and framework for further researchers to build upon in other software packages, utilising different skill sets and abilities. 


\section{FUTURE WORKS}

There are a number of projects that could be developed from this body of research. One of the main ways that the entirety of the process could be improved is through the implementation of custom code, and scripting. This has been proven as a viable option, and would truly add to the control the designer has over the outcomes, and their construction. An alternative approach may include attempting a similar project with a focus on identifying the strengths and weaknesses of existing Grasshopper plugins.

Additionally, further research would ideally be a team based approach. This research does not necessarily propose that this process needs to be undertaken in an interdisciplinary manner, but rather this could be attempted by a small group of interested architects, designers, or general researchers. This may involve the combining of different skills, including specialisations such as parametric/procedural modelling, texturing/detailing, and virtual reality environments. An individual would struggle to investigate all three of these topics in sufficient depth, but with the combined effort of multiple people this could be an effective means of developing new tools, systems, and ideas. Each of these components would be necessary in order to create a fully immersive and exciting experience.

Future continuations of this project, in particular, may also include direct comparisons to real city environments. In isolation, the forms generated by the systems displayed in this research may be viewed as sterilised representations of real world settings. However, examples such as Tokyo, Barcelona, New York, and even Wellington, could be utilised to make comparisons to further refine the outputs generated by the created systems. These comparisons could take place between GIS data and the established footprints, or extruded forms and real world city environments. If compared using the same graphical format then it is possible that one would not be discernible from the other. 


\section{BIBLIOGRAPHY}

Alexander, C. (1965). A city is not a tree. Architectural Forum, 122(1), 58-62. Retrieved from http://en bp ntu.edu.tw/wp-content/uploads/2011/12/06-AlexanderA-city-is-not-a-tree.pdf

Alexander, C., Ishikawa, S., \& Silverstein, M. (1977).

A Pattern Language: Towns, Buildings, Construction: Oxford University Press.

Al-Jamea, S. (2017). Hollywood's Greatest Trick [Video file].

Retrieved from https://vimeo.com/213520878

Baker, C. (2016). 'No Man's Sky': How Games Are Building Themselves: Rolling Stone Retrieved from http://www.rollingstone.com/culture/news/no-mans-sky-how-gamesare-building-themselves-w433492

Bazalo, F., \& Moleta, T. J. (2015). Responsive Algorithms. Paper presented at the Conference of the Association for Computer-Aided Architectural Design Research in Asia (CAADRIA 2015), Daegu.

Retrieved from http://papers.cumincad.org/cgi-bin/works/Show? caadria2015_237

Biljecki, F., Ledoux, H., \& Stoter, J. (2016). Generation of multi-LOD 3D city models in CityGML with the procedural modelling engine Random3Dcity. International Society for Photogrammetry and Remote Sensing.

http://dx.doi.org/10.5194/isprs-annals-IV-4-W1-51-2016

Bryant, R. (2013). Interview with Henry Goss on hyper-realistic 3D architectural renders. Dezeen Retrieved from https://www dezeen.com/2013/08/12/henry-goss-on-architecturalvisualisations/

CityEngineTV (2016). Esri CityEngine 2016 Highlights [Video file] Retrieved from https://www.youtube.com/watch?v=mDOyml8LbPY

Colvin, G. (2013). Why New York is (still) the center of the business universe: Fortune Retrieved from http://fortune.com/2013/11/11/why-new-york-is-still-the-center-of-thebusiness-universe/

Coorey, B. (2016). Digital Design in 2016: An industry snapshot of Australia: Architecture And Design

Retrieved from http://www.architectureanddesign.com.au/features/featuresarticles/most-popular-digital-design-tools-in-australia

(2017). UNSW Students Generate Dozens of City Designs Through Software Programming: The Institute of Digital Design Australia Retrieved from https://www.idda.com.au/unsw-students-generate-dozens-of-citydesigns-through-software-programming

Davis, D. (2010). Untangling Grasshopper - Part 1: Design patterns.

Retrieved from http://www.danieldavis.com/untangling-Grasshopper-part-1-designpatterns/
(2013). Modelled on Software Engineering: Flexible Parametric Models in the Practice of Architecture. (Doctor of Philosophy), RMIT University.

Retrieved from http://www.danieldavis.com/papers/danieldavis thesis.pdf

Díaz, L. (2012). Villa "La Rotonda" de Andrea Palladio.

Retrieved from http://www.podiomx.com/2012/11/villa-la-rotonda-de-andreazpalladio.htm

Duvall. A. (2016). Taking the Occam Razor Approach to Design: Speckyboy Design Magazine Retrieved from https://speckyboy.com/taking-the-occam-razor-approach-to-design/

Edulearn. (2014). What is Autodesk Maya?: Edulearn

Retrieved from http://www.edulearn.com/article/what_is_autodesk_maya.htm

Esri. (2017). Esri CityEngine - 3D Modeling Software for Urban Environments.

Retrieved from http://www.esri.com/software/cityengine

Fletcher, R. (2000). Golden Proportions in a Great House: Palladio's Villa Emo. Nexus III: Architecture and Mathematics, $73-85$.

Retrieved from https://www.nexusjournal.com/the-nexus-conferences/nexusRetrieved from https://www.n

Flux.io. (2016). Site Extractor: Flux.io

Retrieved from https://flux.io/extractor/

Hello Games (2016). About Us: Hello Games

Retrieved from http://www.hellogames.org/about-us/

Goodreads. (2017). A Pattern Language: Towns, Buildings, Construction: Goodreads. Retrieved from http://www.goodreads.com/work/best_book/77027-a-patternlanguage-towns-buildings-construction

Hoffman, R. (2007). Viewpoint - The Growth of 3D on TV | Computer Graphics World, 30. Retrieved from http://www.cgw.com/Publications/CGW/2007/Volume-30-Issue-3March-2007-/Viewpoint-The-Growth-of-3D-on-TV.aspx

Hussain, T. (2016). No Man's Sky's 18 Quintillion Planets Take Up Just 6 GB on Disc. Retrieved from https://www.gamespot.com/articles/no-mans-skys-18quintillion-planets-take-up-just-6/1 100-6441663/

James, M. (2015, 09 / 06 / 2015). 10 Reasons Why CGl is Getting Worse, Not Better: Rocketstock Retrieved from https://www.rocketstock.com/blog/opinion-10-reasons-why-cgi-isgetting-worse-not-better/

Kilkelly, M. (2016). 5 Ways Computational Design Will Change the Way You Work: ArchDaily Retrieved from http://www. archdaily.com/785602/5-ways-computational-design-willchange-the-way-you-work 
Kluyskens, T. (2016, September 29). September Meetup: Tristan Bunn talking vectors + a Houdini introduction. Lecture presented at Victoria University of Wellington. Wellington

Liao, A. (2015). The 21st-Century Skill Set for Architects: Architect Magazine Retrieved from http://www.architectmagazine.com/practice/the-21 st-century-skillset-for-architects_o

Buffalo Library. (2017). A Pattern language: Towns, Buildings, Construction reviews. Retrieved from https://www.buffalolib.org/vufind/Record/3279/Reviews

Mehaffy, M. (2016). A Pattern Language Explained: Permaculture Retrieved from https://www.permaculture.co.uk/articles/pattern-language-explained

Miller, N. (2016). Should architects learn to code?: Building Design + Construction Network Retrieved from https://www.bdcnetwork.com/blog/should-architects-learn-code

Müller, P., Wonka, P., Haegler, S., Ulmer, A., Van Gool, L., Müller, P... .. Van Gool, L. (2006) Procedural modeling of buildings. Paper presented at the ACM Transactions on Procedural modes (TOG)

Retrieved from http://dl.acm.org/citation.cfm2id=1179352,114193

O'Hara, R. J. (2016). Christopher Alexander: A Pattern Language (RJO's Reviews). Retrieved from http://rjohara.net/reviews/alexander

Peter, D. (2014). Autodesk Maya Modeling Tutorial - Create a Building with Python [Video file] Retrieved from https://www.youtube.com/watch? $v=-\mathrm{O}$ sBoLSS50

Qurik, V. (2013). Are Renderings Bad for Architecture?

Retrieved from http://www.archdaily.com/383325/are-renderings-bad-forarchitecture/

Rutten, D. (2012). What are random seed values?

Retrieved from http://www.Grasshopper3d.com/forum/topics/what-are-randomseed-values

SideFX. (2017). Film/TV | SideFX Retrieved from https://www.sidefx.com/filmtv/

Smelik, R. M., Tutenel, T., Bidarra, R., \& Benes, B. (2014). A Survey on Procedural Modelling for Virtual Worlds. Computer Graphics Forum, 33(6), 31-50 Retrieved from http://onlinelibrary.wiley.com/doi/10.1111/cgf.12276/full

Tabarsi, A. (2016). Advice on Becoming a VFX Artist: Brown Bag Labs Retrieved from http://www.brownbagfilms.com/labs/entry/advice-on-becoming-avfx-artist
Welch, C. Moleta, T. J., \& Moloney, J. (2014). Selective Interference: Emergent complexity informed by programmatic, social and performative criteria. Paper presented at the ACADIA 14: Design Agency, Los Angeles.

Retrieved from http://papers.cumincad.org/data/works/att/acadial4_719.content. pdf

White, C. (2006). Interview with Chris White on King Kong: CG Architect

Retrieved from http://www.cgarchitect.com/2006/04/interview-with-chris-white-onking-kong

(2016, June 23). Chris White (Weta Digital) talks Generative Design in Film. Lecture presented at Victoria University of Wellington. Wellington.

Wonka, P., Wimmer, M., Sillion, F., Ribarsky, W., Wonka, P., Wimmer, M., .. Ribarsky, W. (2003). Instant architecture. Paper presented at the ACM Transactions on Graphics (TOG), San Diego, California.

Retrieved from http://dl.acm.org/citation.cfm?id=1201775.882324 


\section{FIGURE LIST}

ALL IMAGES AUTHOR CREATED, UNLESS OTHERWISE STATED

Figure 1.02: Grasshopper canvas visualised as communicative diagram

Figure 1.01: Image from Grasshopper canvas

Figure 1.03: Analysis of First Grasshopper canvas

Figure 1.04: Analysis of final Grasshopper canvas

Figure 1.05: Illustration of Christopher Alexander's 'Positive Outdoor Space' (Noiseux, 2011)

Figure 2.01: Single section output

Figure 2.02: Single \& Rotated section

Figure 2.03: Single, Rotated \& Mirrored sections

Figure 2.04: Analysis of Palladio's Villa Capra (Podiomx, 2012)

Figure 2.05: Palladio's Villa Capra (Baver, 2007)

Figure 2.06: Palladio's Villa Capra (" ")

Figure 2.07: Plan \& Perspective line drawings of Grasshopper output version 01

Figure 2.08: Elevation \& Perspective clay renders of output 01

Figure 2.09: Plan \& Perspective line drawings of output 02

Figure 2.10: Elevation \& Perspective clay renders of output 02

Figure 2.11: Analysis diagram of Classical Column orders (Rouchell, 2013)

Figure 2.12: Different columns types output by the Grasshopper system

Figure 2.13: Composition of proportionate generation of Corinthian columns

Figure 2.14: Failed procedural lonic \& Corinthian column outputs

Figure 2.15: Procedurally generated creatures for No Man's Sky (Hello Games, 2016)

Figure 2.16: Procedurally generated vehicles for No Man's Sky (" "

Figure 2.17: Composition of procedurally generated Tuscan columns

Figure 2.18: Composition of procedurally generated Doric columns

Figure 2.19: Composition of procedurally generated lonic columns

Figure 2.20: Composition of procedurally generated Corinthian columns

Figure 2.21: Composition of procedural column shaft outputs

Figure 2.22: Composition of procedural columns outputs - Standard Version

Figure 2.23: Composition of procedural columns outputs - Mirrored Standard Version

Figure 2.24: Composition of procedural columns outputs - Mirrored standard version, 0.25 Changes to scaling

Figure 2.25: Composition of procedural columns outputs - Halved rotation values \& reduced spheres
Figure 3.01: On set of King Kong (Universal Studios, 2006)

Figure 3.02: Final shot in King Kong film (" " "

Figure 3.03: Image showing composition of building data (" ")

Figure 3.04: NYC Blocks extracted with Flux.IO

Figure 3.05: Outputs from basic city definition

Figure 3.06: Outputs from basic city extrusion definition

Figure 3.07: Composition of attractor based extrusion outputs

Figure 3.08: Composition of early footprint generator outputs

Figure 3.09: Construction logic for creating blocks

Figure 3.10: Composition of outputs from block creator

Figure 4.01: Construction logic for creating Iteration One blocks

Figure 4.02: Compilation of Iteration One outputs - Internal scaling 0.1 - 0.5

Figure 4.03: Compilation of Iteration One outputs - Varied internal scaling

Figure 4.04: Construction logic for creating Iteration Two blocks

Figure 4.05: Compilation of Iteration Two outputs - Standard

Figure 4.06: Compilation of Iteration Two outputs

Figure 4.07: Compilation of Iteration Two outputs

Figure 4.08: Compilation of Iteration Two outputs

Figure 4.09: Compilation of Iteration Two outputs

Figure 4.10: Construction logic for creating Iteration Three blocks

Figure 4.11: Compilation of outputs - Standard version

Figure 4.12: Compilation of outputs - Increased minimum / decreased maximum width Figure 4.13: Compilation of outputs - Smaller base surface

Figure 4.14: Compilation of outputs - Small base surface

Figure 4.15: Compilation of outputs - Length / Width scaling

Figure 4.16: Compilation of outputs - Length / Width / Depth scaling

Figure 4.17: Compilation of outputs - Extrusion from page 065 outputs

Figure 4.18: Compilation of outputs - Extrusion from page 066 outputs

Figure 4.19: Construction logic for creating Iteration Four blocks

Figure 4.20: Compilation of outputs - centre scaling compariso 
Figure 4.21: Compilation of outputs - Standard version

Figure 4.22: Compilation of outputs - Increased width: depth ratio

Figure 4.23: Compilation of outputs - Reduced minimum / maximum building width

Figure 4.24: Compilation of outputs - Increased minimum / maximum building width

Figure 4.25: Compilation of outputs - Significantly decreased surface size

Figure 4.26: Compilation of outputs - Decreased surface size

Figure 5.01: Single output from extruded block generator, Seed 10

Figure 5.02: Compilation of Extruded Block Generator outputs

Figure 5.03: Anatomy of a block: Structure, Seed 07

Figure 5.04: Windows

Figure 5.06: Building Volumes \& Verandahs

Figure 5.05: Rooftops

Figure 5.07: Varying floor heights on Seed 30 model

Figure 5.08: Small scale city generator seed distribution

Figure 5.09: Small scale city generator output, Seed 02

Figure 5.10: Small scale city generator output, 2×2 Blocks, Seed 03

Figure 5.11: Small scale city generator output, $2 \times 2$ Blocks, Seed 04

Figure 5.12: Small scale city generator output, 3×3 Blocks, Seed 03

Figure 5.13: Small scale city generator output, 3×3 Blocks, Seed 04

Figure 5.14: Small scale city generator output, 3×3 Blocks, 6-18 Floor Height, Seed 03

Figure 5.15: Small scale city generator output, 3×3 Blocks, 6-18 Floor Height, 200\% zoom, Seed 03

Figure 5.16: Compilation of extruded block generator with building height awareness outputs

Figure 5.17: Window optimisation comparison images

Figure 5.18: Building volume optimisation comparison images

Figure 5.19: Screenshot of Human.Ul interface, within Grasshopper

Figure 5.20: Compilation of footprint generator outputs 2.0 to 5.0 maximum width/depth scaling

Figure 5.21: Compilation of footprint generator outputs. Same seed on different bases

Figure 6.01: Construction of city generator system

Figure 6.02: City generator output, Seed 01

Figure 6.03: Compilation of city generator outputs, lowest possible park/carpark generation

Figure 6.04: Compilation of city generator outputs, increasing park/carpark generation
Figure 6.05: Compilation of city generator outputs, lowest possible park/carpark generation, varied building width range

Figure 6.06: Compilation of city generator outputs, building scaling value added

Figure 6.07: City generator output, building scaling range 2.0 - 6.5 added, Seed 02

Figure 6.08: Compilation of city generator outputs, including jittered values and varied extrusion ranges, Seed 02

Figure 6.09: Compilation of city generator outputs, including varied jittered values and fixed extrusion range, Seed 02

Figure 6.10: Compilation of city generator outputs, including fixed jittering values, fixed extrusion range, and varied attractor point placement, Seed 02

Figure 6.11: Compilation of city generator outputs, including fixed jittering values, fixed extrusion range, and varied attractor point placement, 200\% zoom, Seed 02

\section{IMAGE REFERENCES}

Baver, S. (2007). Palladios Villa Rotonda, Veneto, Italy. Front. [Photograph] Retrieved from https://commons.wikimedia.org/wiki/File:Villa_Rotonda_front.jpg

(2007). Palladios Villa Rotonda, Veneto, Italy. Side. [Photograph] Retrieved from https://commons.wikimedia.org/wiki/File:Villa_Rotonda_side.jpg

Hello Games (2016). No Man's Sky Creature Comparison [Digital]. Retrieved from http://image noelshack.com/fichiers/2014/34/1408794485- sans-titre. png

(2016). No Man's Sky Ship Comparison [Digital]. Retrieved from https://pbs.twimg.com/media/BvCollillQAAxtnM.png:large

Noiseux, M. (201 1). Illustration of Christopher Alexander's 'Positive Outdoor Space' [Drawing] Retrieved from https://serenityinthegarden.blogspot.co.nz/201 1/04/patternlanguage-by-christopher.htm

Podiomx (Original author unknown) (2012). Analysis of Villa Capra [Drawing] Retrieved from http://www podiomx.com/2012/11/villa-la-rotonda-de-andreapalladio.htm

Rouchell, M. (2013). The Classical Orders of Architecture [Drawing]. Retrieved from https://mrouchell.files.wordpress.com/2013/03/classical-orders 1.jpg

Universal Studios (2006). [Digital]. Retrieved from http://www.cgarchitect.com/2006/04/interview-with-chris- hite-onking-kong 